\title{
Lettura \\ e dispersione
}

A cura di

Irene Dora Maria Scierri

Marco Bartolucci

Rosario Salvato

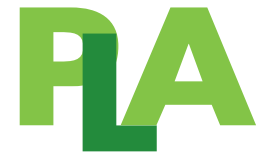

Processi

e Linguaggi

dell'Apprendimento 


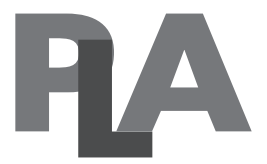

Processi

e Linguaggi

dell'Apprendimento

\section{Direzione: Roberto Trinchero}

\section{Comitato direttivo}

Funzioni: accoglienza delle proposte di pubblicazione e prima scrematura

Barbara Bruschi, Renato Grimaldi, Roberto Farné, Alberto Parola, Daniela Robasto, Barbara Sini, Simona Tirocchi

\section{Comitato Scientifico}

Funzioni: referaggio anonimo, con doppio cieco, mediante sistema on line

Michele Baldassarre, Federico Batini, Guido Benvenuto, Giovanni Bonaiuti, Vincenzo Bonazza, Antonio Calvani, Gianna Cappello, Lucia Chiappetta Cajola, Cristina Coggi, Barbara Demo, Luciano Di Mele, Piergiuseppe Ellerani, Ivan Enrici, Damiano Felini, Adelaide Gallina, Marco Gui, Sara Nosari, Alessandro Perissinotto, Maria Ranieri, Paola Ricchiardi, Emanuela Torre, Carla Tinti, Giuliano Vivanet, Tamara Zappaterra.

La Collana accoglie studi teorici, storico-comparativi ed empirico-sperimentali riguardanti i processi e i linguaggi dell'apprendimento dalla primissima infanzia alla "grande anzianită". I testi proposti sono volti a indagare "come si apprende" nelle varie età della vita e come è possibile mettere in atto processi di formazione efficaci nel promuovere apprendimento, tenendo conto del dibattito contemporaneo in pedagogia, didattica, psicologia cognitiva, neuroscienze. In quest'ottica, i testi proposti esplorano i metodi, le strategie, le tecniche e gli strumenti efficaci nei percorsi di educazione, istruzione e formazione, scolastica ed extrascolastica, lungo tutto l'arco della vita.

Oggetti di interesse sono quindi l'educazione e la formazione improntate dall'evidenza quantitativa e qualitativa, I'apprendimento esperienziale in diversi contesti - dal gioco spontaneo del bambino all'interazione mediata dai social network -, i linguaggi mediali per l'apprendimento e le tecnologie in grado di promuoverIo, il potenziamento cognitivo come strumento per affrontare un vasto speftro di bisogni educativi, la gamification, la robotica educativa, la giocomotricità e le sinergie tra apprendimento cognitivo e motorio, lo storytelling, i prodotti mono e multimediali per l'infanzia e il gioco educativo nelle sue varie forme e accezioni. La collana accoglie contributi di studiosi italiani e di altri paesi , sotto forma di monografie, volumi collettanei, rapporti di ricerca, traduzioni, descrizioni di esperienze e sperimentazioni in contesti scolastici ed extrascolastici.

Il Comitato direttivo e il Comitato scientifico intendono promuovere attraverso la collana un ampio, aperto e proficuo dibattito tra ricercatori, insegnanti, educatori e tutti gli studiosi che siano interessati ai processi e ai linguaggi dell'apprendimento nelle varie età della vita.

Ogni volume è sottoposto a referaggio con modello "doppio cieco". 


\section{OPEN ACCESS \\ la soluzione FrancoAngeli}

Il presente volume è pubblicato in open access, ossia il file dell'intero lavoro è liberamente scaricabile dalla piattaforma FrancoAngeli Open Access (http://bit.ly/francoangeli-oa).

FrancoAngeli Open Access è la piattaforma per pubblicare articoli e monografie, rispettando gli standard etici e qualitativi e la messa a disposizione dei contenuti ad accesso aperto. Oltre a garantire il deposito nei maggiori archivi e repository internazionali $\mathrm{OA}$, la sua integrazione con tutto il ricco catalogo di riviste e collane FrancoAngeli massimizza la visibilità, favorisce facilità di ricerca per l'utente e possibilità di impatto per l'autore.

Per saperne di più:

http://www.francoangeli.it/come_pubblicare/pubblicare_19.asp

I lettori che desiderano informarsi sui libri e le riviste da noi pubblicati possono consultare il nostro sito Internet: $w w w$ francoangeli.it e iscriversi nella home page al servizio "Informatemi" per ricevere via e-mail le segnalazioni delle novità. 


\title{
Lettura \\ e dispersione
}

\author{
A cura di \\ Irene Dora Maria Scierri \\ Marco Bartolucci \\ Rosario Salvato
}

\section{FrancoAngeli OPEN 2 ACCESS}


Progetto di gruppo Ricerca di base 2015 - "La dispersione in Umbria: definizione del perimetro e costruzione di un sistema di prevenzione", finanziato con il fondo Ricerca di base anno 2015 (quota gruppo).

Copyright (C) 2018 by FrancoAngeli s.r.1., Milano, Italy.

L'opera, comprese tutte le sue parti, è tutelata dalla legge sul diritto d'autore ed è pubblicata in versione digitale con licenza Creative Commons Attribuzione-Non Commerciale-Non opere derivate 3.0 Italia (CC-BY-NC-ND 3.0 IT)

L'Utente nel momento in cui effettua il download dell'opera accetta tutte le condizioni della licenza d'uso dell'opera previste e comunicate sul sito http://creativecommons.org/licenses/by-nc-nd/3.0/it/legalcode 


\section{Indice}

Introduzione, di Federico Batini

pag. $\quad 9$

\section{Parte I - Lettura e dispersione scolastica}

Tra lettura e dispersione

di Federico Batini

II contributo della lettura contro la dispersione scolastica

di Agnese Rosati

Le pratiche di lettura nelle scuole dell'infanzia e primarie umbre

di Marco Bartolucci e Adriana Timpone

Lettura e intercultura nelle scuole dell'infanzia e primarie dell'Umbria

di Roberta Balloni, Ylenia Di Vito e Laura Lana 
Dispersione scolastica: visioni a confronto. Indagine sulla percezione della dispersione tra studenti, insegnanti e genitori delle scuole secondarie umbre

L'atteggiamento dei docenti nei confronti della dispersione scolastica

di Cecilia Bini, Leonardo Fasi, Elisa Morini e

Alessandra Sisto

La dispersione universitaria e il processo di inclusione degli studenti con disabilità e/o con DSA

di Laura Arcangeli e Moira Sannipoli

\section{Parte II - Pratiche e politiche per prevenire la dispersione}

Se me ne vado, ci saranno problemi...

La mancanza di adesione alle offerte formative in Danimarca, in una prospettiva di capabilities

di Christian Christrup Kjeldsen

La dispersione scolastica e le politiche territoriali di welfare: prevenzione, contrasto e azioni di recupero. Dal piano europeo e nazionale alla realtà umbra

di Rosario Salvato

Una valutazione per il successo formativo di Cristiano Corsini 
Le nuove tecnologie per la prevenzione della dispersione scolastica: le possibilità offerte dal digitale alla lettura

di Floriana Falcinelli

pag. 211

Insegnanti abili ed efficaci: una risorsa per contrastare l'abbandono scolastico

di Mina De Santis e Annalisa Morganti

» 219

Dispersione scolastica: lo studio, un dovere e un diritto

di Giovanna Farinelli

» 231

Profili autori

» 247 


\title{
Introduzione
}

\author{
di Federico Batini
}

"La lettura, come questo libro afferma con convinzione,

è fondamentale aiuto per vivere degnamente.

Né dovrebbe essere necessario difendere la necessità di vivere umanamente o secondo ragione, anche se talvolta v'è chi lascia sospettare il contrario." (M.J. Adler, Come si legge un libro, p. 5, Armando, 1964, edizione originale, 1940).

La dispersione scolastica nelle secondarie di II grado raggiunge cifre impressionanti. Negli ultimi dieci anni, su sei milioni (6.114.644) di studenti iscritti al I anno delle secondarie di secondo grado negli istituti statali, un milione e 750 mila studenti (1.744.142) non è arrivato all'ultimo anno. Il 28,5\% disperso, non pervenuto, "perso" dal sistema di istruzione statale.

(Fonte: Tuttoscuola, 30 gennaio 2018)

Affrontare il tema della dispersione scolastica rappresenta, spesso, un azzardo. L'azzardo si situa nel rischio di venire fraintesi e di non veder identificato, con precisione, l'oggetto della propria ricerca. Problema secondario a fronte dell'affronto che subiscono i ragazzi investiti dal problema, il rischio di "scomparire", di non risultare. Come avremo modo di leggere nel volume, infatti, la confusione circa il significato da dare all'espressione pare aumentare anziché diminuire e sembra difficile, oggi, reperire documenti o rapporti, anche istituzionali, che prendano in esame tutte le componenti che vanno a costituire le dimensioni di questo costrutto complesso. Pare sia diventato difficile rispondere a due semplici domande: Che cos'è, insomma, la dispersione scolastica? E di conseguenza: qual è la situazione attuale in Italia (e nei livelli locali) e negli altri paesi in proposito?

La dispersione scolastica è stata definita, nel tempo, in vari modi e attraverso differenti approcci di quantificazione. Definirne i confini e confrontarsi con le definizioni non è un esercizio di stile, riguarda il destino di centinaia di migliaia di studenti. Occorre, allora, evitare di cadere in fraintendimenti. Come apprendiamo anche in questo volume, la pressione comunitaria 
sugli Stati Membri a rendere conto di coloro che non raggiungono titoli successivi alla licenza media, rilevando il dato ex post, nei soggetti compresi tra 18 e 24 anni, costituisce un osservatorio distorto. Un po' come stabilire la gravità degli effetti di un'epidemia contandone i morti e senza adottare prevenzione, senza monitorare e curare chi mostra i primi sintomi, senza definire quali condizioni ambientali limitino la diffusione dell'epidemia stessa. Si tratta, cioè, di uno sguardo ex post che non sollecita politiche preventive (e quando lo fa le politiche scelte non dialogano con le evidenze empiriche della ricerca) né, per completezza, pare sollecitare politiche curative adeguate (interventi massivi sui giovani adulti).

La dispersione, intanto, va avanti, noncurante dei discorsi e delle petizioni di principio, si concretizza nell'abbandono scolastico, nelle ripetenze, nelle non frequenze e frequenze parziali. Viene misurata, invece, spesso, attraverso il solo abbandono formalizzato. Chi non frequenta le scuole, o frequenta solo scuole di un certo tipo, non coglie la tragica ironia che si cela dietro queste pratiche. Di fatto chi smette di andare a scuola o frequenta saltuariamente, nella maggior parte dei casi non formalizza in alcun modo il proprio abbandono. Dove finiscono i ragazzi che smettono di frequentare senza andare a scuola, con i genitori, a firmare il "foglio di rinuncia"? Risulteranno iscritti alla stessa classe l'anno successivo? E chi ha partecipato di tanto in tanto? Le ripetenze sono un importantissimo campanello di allarme, perché consentono di esercitare una previsione sull'anno immediatamente successivo (come è noto le ripetenze moltiplicano le probabilità di abbandono) e, se analizzate nel dettaglio, forniscono indicazioni ulteriori circa la loro natura ("non scrutinato per raggiunti limiti di assenza" o per "impossibilità di accedere a una valutazione" sono indicatori espliciti di non frequenza). Sarebbe terribile (e miope) cedere alla tentazione di modificare le modalità di contabilizzazione per presentare numeri migliori.

Non è semplice ma non è impossibile monitorare la dispersione, specie in era di registro elettronico. Occorre tenere sotto controllo abbandoni, ritiri, ripetenze e non frequenze mentre si svolgono, e definire protocolli di intervento di area. Occorre assumersi una responsabilità educativa e di istruzione e non nascondersi dietro le cause esterne alla scuola. La scuola deve essere inclusiva e promuovere gli apprendimenti di tutti. C'è molto che non funziona e che non permette a ciascun insegnante di agire al meglio, ma non è una scusa nei confronti della propria responsabilità. Dove gli insegnanti, magari in dialogo con il terzo settore e la ricerca, sono stati disponibili a rimettere in discussione pratiche didattiche e valutative, a definire intenzionalmente obiettivi di apprendimento, i risultati, misurati seguendo più logiche $\mathrm{e}$ 
approcci, ci sono stati e sono evidenti (si rimanda ai riferimenti indicati e esplicitati nei primi capitoli del volume).

In questo volume la dispersione scolastica viene messa accanto alla "lettura". La lettura è un'altra area di ricerca importante per molti tra gli autori di questo volume e costituisce un antidoto importante all'insuccesso formativo, viatico di ogni dispersione.

Le sperimentazioni di questi anni ci hanno permesso di valutare gli straordinari effetti di sessioni di lettura ad alta voce, accompagnate o meno da pratiche didattiche attive. Anche la lettura ad alta voce (senza altro tipo di interventi), protratta per soli 40 giorni, per circa un'ora al giorno, ha mostrato, sottoposta a misurazioni con disegni sperimentali o quasi sperimentali, un'incidenza davvero particolare su molte dimensioni cognitive, emotive, relazionali. Ha mostrato correlazione con il progresso delle competenze relative alla produzione e comprensione di testi e, più in generale, con lo sviluppo di abilità e competenze legate al successo formativo.

Questo volume però racconta anche un'altra storia: una storia complessa che intreccia didattica e ricerca. Da oltre dieci anni nella didattica universitaria, presso l'Università degli Studi di Perugia, Dipartimento FISSUF, nei corsi di Metodologia della ricerca educativa, dell'osservazione e della valutazione (corso di laurea triennale in Scienze dell'Educazione) e di Pedagogia sperimentale (corso di laurea a ciclo unico in Scienze della Formazione Primaria) ${ }^{1}$ tentiamo, insieme a preziosi collaboratori della cattedra, di rendere più vivi, partecipati, capaci di produrre apprendimenti reali, i corsi. Per far questo ogni anno organizziamo una serie di prove in itinere tra le quali c'è sempre un'attività di ricerca concreta (condividiamo anche, da subito, modalità e criteri di valutazione). In questo volume vengono presentati i dati relativi a due ricerche realizzate insieme a tutti gli studenti dei corsi, organizzati in gruppi. Hanno costruito un quadro teorico, abbiamo definito insieme il campione, abbiamo costruito gli strumenti di rilevazione (poi rivisti da noi), abbiamo negoziato fino ad arrivare a strumenti condivisi, i gruppi hanno avuto assegnate scuole di riferimento (e hanno sperimentato la difficoltà di moltissimi rifiuti) per fare le rilevazioni. Ciascun gruppo ha poi organizzato i propri dati e li ha analizzati. A tutti i gruppi sono stati poi restituiti i dati complessivi analizzati da noi. Ciascuna scuola coinvolta ha avuto accesso a tutti i dati raccolti ed alle loro elaborazioni, per scuola e complessive.

${ }^{1}$ Fino all'anno accademico 2016/2017 anche nel corso di Pedagogia Sperimentale per il corso di laurea in Scienze e Tecniche Psicologiche e dei Processi Mentali. 
Due gruppi hanno voluto proseguire l'attività, incoraggiati da noi, e hanno presentato una relazione al convegno organizzato presso il Dipartimento FISSUF e, supportati e con una serie di feedback, presentano due capitoli in questo volume 2 .

Nel primo capitolo tento di tracciare un rapporto complessivo tra lettura e dispersione, evidenziando i rischi di una contabilità ex post della dispersione e cercando di mostrare come la lettura possa costituire, all'interno del sistema di istruzione, una piccola strategia (a costo quasi zero) con grandissime potenzialità, a partire dalla primissima infanzia (con la grande potenzialità offerta dall'apertura di un'attenzione alla fascia $0-6$ ). La rassegna di alcuni training quasi-sperimentali realizzati negli ultimi anni e delle diverse misurazioni e valutazioni compiute sostiene la fondatezza del rapporto e la potenzialità della lettura ad alta voce nel sistema di istruzione.

Agnese Rosati ci conduce tra le finalità educative della lettura individuandole nella formazione di un pensiero libero, autonomo, immune ai pregiudizi e ai luoghi comuni. Nella promozione della lettura viene individuato un impegno necessario, attraverso progetti, manifestazioni, eventi e teso al coinvolgimento delle famiglie nell'utilizzo e frequentazione di libri per ogni fascia di età. Successivamente vengono identificati ulteriori motivi per leggere e vengono presentate, secondo una prospettiva teorica, le ragioni di un'utilità della lettura nella prevenzione della dispersione.

Marco Bartolucci e Adriana Timpone presentano gli esiti della ricerca sulle pratiche di lettura nelle scuole dell'infanzia e primarie umbre (realizzata con le studentesse e gli studenti di Scienze della Formazione Primaria).

Nel capitolo successivo Roberta Balloni, Ylenia Di Vito e Laura Lana presentano un affondo sulla dimensione interculturale, relativo alla stessa ricerca.

Irene Scierri e Mariangela Nitti presentano invece i risultati della ricerca sulla percezione della dispersione scolastica secondo studenti, genitori e insegnanti nelle scuole secondarie di primo e secondo grado umbre (realizzata con le studentesse e gli studenti di Scienze dell'Educazione).

Nel capitolo successivo Cecilia Bini, Leonardo Fasi, Elisa Morini e Alessandra Sisto approfondiscono l'atteggiamento dei docenti nei confronti della dispersione scolastica.

\footnotetext{
${ }^{2}$ Per noi si tratta di un risultato importante. Un tentativo di coinvolgimento attivo di gruppi di studenti delle secondarie di secondo grado è stato fatto, poi i tempi burocratici dell'alternanza non coincidevano con il calendario dei corsi e abbiamo dovuto sospendere l'esperienza, riproveremo l'anno prossimo.
} 
Concludono la prima parte Laura Arcangeli e Moira Sannipoli che presentano la dispersione universitaria degli studenti con disabilità e degli studenti con DSA. Dopo un'introduzione problematica sul monitoraggio e gli andamenti della dispersione in Università viene offerta un'interessante panoramica su DSA e carriera universitaria (interventi di orientamento e riorientamento; interventi su metodi di studio; interventi su didattica universitaria tesi all'inclusione dei soggetti con DSA). Il contributo infine fornisce un dettagliato resoconto dell'esperienza perugina.

La seconda parte viene aperta dal collega danese Christian Christrup Kjeldsen che ci parla di una realtà del tutto diversa. Il problema delle non frequenze e le strategie messe in campo per arginare il fenomeno in Danimarca, adottando una prospettiva sociale e ponendo un problema di equità e giustizia del sistema educativo. Le domande del collega interrogano fortemente anche il sistema di istruzione italiano. Come potremmo rispondere, infatti, a questa sua affermazione: "Se si dovesse giudicare - da un punto di vista di giustizia sociale - un sistema educativo, il responso potrebbe apparire piuttosto chiaro guardando alle politiche inerenti gli studenti più svantaggiati, provenienti da famiglie con uno status socio-economico basso. Qual è la loro libertà di vivere la vita a cui attribuiscono valore? Quali scelte vengono date - se ne vengono date?" Sappiamo, infatti, che in Italia gli studenti svantaggiati sono le prime "vittime" della dispersione scolastica. L'approccio per capabilities, proposto dal collega danese, prevede non soltanto di indagare la possibilità formale, ma quella sostanziale, compresa la strumentazione atta a muovere la volontà di farlo, pare rivelarsi un approccio promettente per distinguere le opportunità formali da quelle sostanziali e dunque indagare più a fondo sull'accesso reale di un sistema di istruzione. Il contributo è poi offerto al lettore italiano con l'integrazione di una preziosa appendice sul sistema di istruzione danese.

Rosario Salvato (co-curatore del volume) ci propone un'approfondita analisi dei documenti istituzionali circa le politiche di welfare dal livello internazionale sino a quello nazionale e regionale umbro. Riflettendo sulle varie linee guida, emanate a diversi livelli, le politiche di welfare vengono rilette alla luce del fenomeno della dispersione scolastica.

Cristiano Corsini riflette sul momento della valutazione e di come la valutazione stessa, spesso al centro delle decisioni di abbandono degli studenti, possa, diventare strumento di successo formativo. Quale valutazione? La valutazione autentica che si connette, esplicitamente, a motivazione e apprendimento degli studenti. La valutazione, insomma, che si trasforma da momento critico a fattore preventivo della dispersione scolastica. 
Floriana Falcinelli ci propone le indicazioni emergenti dalla ricerca educativa riguardo al rapporto tra nuove tecnologie e apprendimenti, suggerendo che un uso corretto di nuovi media e nuove tecnologie possa, attraverso le potenzialità compensative, comunicative, di mediazione insite in questi strumenti, costituire un'opportunità di incremento del successo formativo e dunque di prevenzione della dispersione. L'introduzione alla didattica digitale e al superamento di alcuni falsi miti costituiscono ulteriori motivi di interesse.

Mina De Santis e Annalisa Morganti ci propongono un insegnante "abile ed efficace" come antidoto alla dispersione. Viene approfondito il ruolo delle competenze emotive e sociali che l'insegnante deve sviluppare al fine di essere in grado, anche con i suoi atteggiamenti, di produrre una didattica inclusiva capace di raggiungere risultati e non escludere. In coerenza con questo approccio viene poi sottolineato il ruolo fondamentale delle didattiche attive in relazione alla lotta alla dispersione.

Giovanna Farinelli oltre a offrirci una precisazione linguistica circa il termine dispersione scolastica ne traccia un interessante relazione diacronica (leggendone gli sviluppi in termini di inclusione sociale) con il diritto all'istruzione e con i fondamenti normativi al quale si lega. Il contributo giunge sino all'analisi dei documenti più recenti del MIUR (sempre in dialogo con i documenti europei e internazionali) e alle proposte di soluzione.

Questo volume, in ideale continuità con il volume Dispersione scolastica: ascoltare i protagonisti per comprenderla e prevenirla (Franco Angeli, 2016), costituisce un ulteriore output di un processo di ricerca continuo.

La dispersione scolastica è un tema particolare attorno al quale si è aggregato un ampio gruppo di ricerca che, dopo un impulso iniziale della cattedra di Pedagogia Sperimentale, ha rapidamente allargato i propri confini, partendo dall'area educativa del Dipartimento FISSUF dell'Università degli Studi di Perugia, per coinvolgere poi altri settori scientifico disciplinari dello stesso dipartimento, collaborazioni con altri dipartimenti ed Atenei e poi con colleghi di altre nazioni. In questo senso va letta la recente nascita, nel 2017, di "IReNE" International Research Network "Nobody Excluded" coordinata da chi scrive.

La lettura è un'altra area di ricerca che all'interno del Dipartimento FISSUF sta trovando uno spazio sempre maggiore. All'uscita di questo volume dovrebbe corrispondere l'avvio di un progetto di sperimentazione nazionale circa gli effetti di training di lettura in scuole primarie e secondarie di primo grado. 
Il volume trova un suo ideale complemento nella piattaforma: indagineumbria.dispersione.it (realizzazione Mariangela Nitti). La piattaforma, navigabile, consente di vedere i dati relativi all'indagine realizzata sulla percezione della dispersione tra studenti, genitori e insegnanti, permette di ispezionare i dati scuola per scuola, verificare le differenze tra diverse tipologie di rispondenti e, come il volume, è open access.

Il ringraziamento ai curatori del volume Irene Scierri, Marco Bartolucci, Rosario Salvato è d'obbligo, ma non formale, anche per essersi, forse avventatamente, rivolti a me per l'introduzione. Spero di non aver troppo "sciupato" un volume davvero utile e importante.

Il ringraziamento agli studenti tutti, per essersi lasciati coinvolgere, dopo comprensibili resistenze, mi riempie di gioia. Una menzione particolare per quelli così tenaci da arrivare sino alla pubblicazione, grazie. I ringraziamenti vanno anche a tutti i colleghi dell'area educativa del Dipartimento FISSUF coinvolti nel volume e a quelli non coinvolti ma che ci hanno sostenuto nella ricerca e nelle fasi successive. Il lavoro ri-iniziato negli ultimi anni, insieme, sta producendo frutti. Cristiano Corsini è ormai un amico oltre che un collega con cui lavorare insieme, lo ringrazio per la sua serietà scientifica e la sua umanità. Ringrazio il collega Christian Christrup Kjeldsen per il rilevante contributo fornitoci e per l'apertura di un dialogo di ricerca che credo fecondo. Un ringraziamento particolare a un gruppo di cui sarebbe lungo elencare i meriti: Irene Scierri, Marco Bartolucci, Giulia Toti, Adriana Timpone, Alberto Santoro, Mariangela Nitti.

Venere (AR), aprile 2018

Federico Batini 


\section{Parte I}

\section{Lettura e dispersione scolastica}




\title{
Tra lettura e dispersione
}

\author{
di Federico Batini
}

\section{Introduzione: uno strano binomio}

Lettura e dispersione scolastica costituiscono uno strano binomio, quasi un ossimoro. In modo e con tempi diversi sono state, entrambe, al centro dell'interesse delle ricerche di area educativa (e non solo), entrando, però, difficilmente in dialogo.

Il tipo di relazione che si crea è, in effetti, una relazione particolare, inversamente proporzionale, un po' come se il discorso suonasse "dove cresce la presenza della lettura e di pratiche di lettura, la dispersione diminuisce". L'affermazione può apparire eccessiva, certo però che si può osservare, senza difficoltà, come lettura precoce e successo formativo siano associati in più ricerche ${ }^{2}$. Molti studi in letteratura hanno permesso di registrare, a seguito di interventi di lettura ad alta voce, un incremento sia del vocabolario "attivo", i termini usati nel linguaggio quotidiano, che di quello "passivo",

\footnotetext{
${ }^{1}$ Questa relazione, dalle nostre osservazioni, è così forte da rubricarla al rango di ipotesi: la pratica della lettura quotidiana sarebbe cioè in grado di esercitare un effetto moderatore sulla dispersione scolastica.

${ }^{2}$ Riprendendo la definizione di Pontecorvo (Pontecorvo e Pontecorvo, 1986, p. 190): «L'abilità di lettura è stata, ed è tuttora, considerata la base essenziale per il successo scolastico tanto che spesso l'istruzione elementare ruota, si può dire, tutta intorno a questo obiettivo. Tuttavia l'attuale interesse per la lettura è anche teorico, nel senso che si aspira oggi a costruire una buona teoria psicologica della lettura». La competenza di lettura, infatti, «comprende un'ampia gamma di competenze cognitive, che vanno da quella, di base, della decodifica, alla conoscenza delle parole, della grammatica e di strutture e caratteristiche linguistiche e testuali più estese, alle conoscenze enciclopediche. Essa comprende anche competenze metacognitive: la capacità di ricorrere ad una pluralità di strategie appropriate nell'elaborazione dei testi e il farlo in modo consapevole. Le competenze metacognitive vengono attivate quando i lettori riflettono circa la loro attività di lettura, la controllano e l'adattano, in vista di un determinato scopo» (OECD, 2009, p. 23).
} 
tutti i termini di cui conosciamo il significato e che siamo in grado di comprendere e utilizzare correttamente in una frase (Duursma, Augustyn e Zuckerman, 2008). La lettura pare essere, quindi, lo strumento principale per favorire lo sviluppo lessicale già dalla primissima infanzia (Isbell et al., 2004). Inoltre, sembra esserci una relazione tra il numero di ore che i genitori trascorrono leggendo ai propri figli e la successiva capacità dei bambini di leggere, di utilizzare frasi complesse, di comprendere significati letterali e inferenziali (Johnston, 2016). Le ricerche indicano come già in età prescolare si possano ottenere benefici sull'attenzione, sulla capacità di ascolto e sulla comprensione del testo con un effect size pari a 0,51 . In età scolare si hanno ripercussioni positive sul piacere della lettura, l'ampliamento del vocabolario e la capacità di fare inferenze (Emili, 2017). Il vantaggio in termini di comprensione e di vocabolario, ottenuto attraverso la lettura o la lettura ad alta voce, si traduce in un vantaggio rispetto al successo formativo perché rende gli studenti maggiormente capaci di comprendere ciò che dice l'insegnante e ciò che c'è scritto nei libri di testo (Matthiessen, 2013). Ulteriori vantaggi riguardano la capacità di ragionamento critico (Batini e Bartolucci, 2016b). Non sarà difficile quindi verificare come al successo formativo corrispondano, di conseguenza, percentuali risibili di dispersione scolastica (chi non ha problemi di rendimento a scuola difficilmente abbandona). La dispersione scolastica, seppur sempre legata a molte cause, è saldamente associata all'insuccesso (Dalton, Gennie e Ingels, 2009; Sabates et al., 2010). È anche nota l'associazione tra dispersione e fallimenti formativi, intesi sia nei termini di ripetenze di anni scolastici (Alivernini e Lucidi, 2011) che di insuccesso selettivo (relativo a una sola disciplina) (Mata, Monteiro e Peixoto, 2012).

La lettura, sebbene comporti benefici che vanno molto al di là di questo, correla significativamente, come abbiamo visto, con il successo formativo. L'associazione, come tenteremo di mostrare in questo contributo, non è quindi peregrina e dovrebbe essere perseguita in modo maggiormente sistemico e strutturato per trarre vantaggio dal potenziale preventivo dell'una nei confronti dell'altra.

\subsection{Una proposta evidence based}

Le ricerche e le esperienze sul campo condotte negli ultimi venti anni ci hanno, infatti, portato a mettere in dialogo queste due aree di interesse della ricerca educativa. Le dimensioni "cognitive" della ricerca (la comprensione e i tentativi di spiegazione) incrociate alle dimensioni "sociali" della ricerca 
stessa (ovvero l'urgenza della domanda posta dalla realtà e il dialogo con le politiche allo scopo di fornirgli orientamento) hanno reso indifferibile il confronto con le pratiche concrete per produrre cambiamento.

Si è quindi posta, progressivamente, con forza, la necessità di proporre un'azione e attenzione preventiva al fenomeno della dispersione scolastica mediante la lettura ad alta voce, proposta in modo sistematico per tutto il primo ciclo di istruzione senza abbandonare la pratica nel grado successivo ${ }^{3}$.

Le dimensioni osservate circa i vantaggi di azioni sistematiche di lettura, anche protratte per brevi periodi, hanno infatti mostrato di poter agire direttamente, o in modo integrato ad altre tipologie di intervento, sulle funzioni cognitive (Sénéchal, 1997; Beck, McKeown e Kucan, 2002; Lane e Wright, 2007; Speer et al., 2009; Horowitz-Kraus, Vannest e Holland, 2013; Hutton et al., 2015; Batini, Bartolucci e De Carlo, 2017), su più aspetti legati al riconoscimento delle emozioni in sé e negli altri (Coplan, 2004; Ronfani et al., 2006; Mar e Oatley, 2008; Aram e Aviram 2009; Aram e Shapira, 2012; Cambi, 2012; Catarsi, 2011), sull'acquisizione di un proprio vocabolario e nell'apprendimento delle tecniche di lettura, sulla comprensione di testi (Sénéchal e LeFevre, 2002; Farrant e Zubrick 2013; Korat, Shamir e Heibal, 2013) e su molto altro.

Queste dimensioni (e gli stessi strumenti di rilevazione utilizzati) hanno mostrato relazioni significative con il successo formativo. Ad esempio il CAS, una batteria utilizzata per rilevare le funzioni cognitive: lo strumento ha mostrato una correlazione significativa tra punteggi al test e risultati scolastici (Naglieri e Rojahn, 2004; Naglieri et al., 2006).

La dispersione scolastica rappresenta uno "spreco": di tempo, di capacità cognitive, di risorse. I ragazzi che si allontanano dal percorso con varie modalità (ritiri, abbandono formale, abbandono di fatto, non frequenza, frequenza saltuaria) costituiscono, rapportati al totale degli iscritti inizialmente, il tasso di abbandono. I ragazzi che ripetono l'anno, perché "bocciati”, rapportati ancora al totale degli iscritti costituiscono, invece, il tasso di ripetenza (Batini e Benvenuto, 2016). La somma del tasso di abbandono e del tasso di ripetenza determina la percentuale di dispersione scolastica.

${ }^{3}$ La proposta di lettura ad alta voce, con cadenza quotidiana all'interno del sistema di istruzione è una proposta di LaAV (www.narrazioni.it). Qualora la proposta venisse presa in considerazione si potrebbe, per le generazioni a venire, effettivamente rallentare la proposta di lettura ad alta voce, nella secondaria di secondo grado, proponendo agli studenti di diventare, a loro volta, lettori per altri (i propri compagni di classe, studenti di classi inferiori, soggetti portatori di bisogni nel territorio etc...). Al momento sarebbe opportuno, invece, avviare una pratica di lettura quotidiana anche alle secondarie di secondo grado. 
La definizione proposta è quella più "stretta" di dispersione, secondo alcuni, infatti dovremmo aggiungere a queste percentuali anche coloro che, decidendo di cambiare scuola o percorso, incorrono, per questo ripensamento, in qualche forma di ritardo (dispersione di tempo) o coloro che pur permanendo nello stesso percorso (magari anche portandolo a termine) non conseguono gli apprendimenti previsti o comunque quelli che sarebbero in grado di raggiungere (dispersione di potenzialità cognitiva) (Batini e Benvenuto, 2016). «La dispersione scolastica deve essere vista non solo come evasione dall'obbligo o abbandono della scuola da parte degli studenti prima della fine del ciclo di studi intrapreso. [...] Il concetto di abbandono scolastico (o school dropping out) è da intendere in rapporto all'idea di scolarizzazione esistente in una determinata società: per i Paesi occidentali una formazione regolare è prevista fino ai 18 anni. C'è dispersione di talenti ogni volta che ci si trova di fronte ad un sentimento di grave malessere che impedisce all'alunno di vivere un'esperienza scolastica pienamente formativa. Si tratta di un problema individuale e sociale.» (Bombardelli, 2001, p. 75). Più in generale la letteratura di settore ha sempre fatto riferimento almeno a tutte quelle cause che fermano o rallentano, impediscono o interrompono lo svolgimento regolare del percorso scolastico (Liverta Sempio, Confalonieri e Scaratti, 1999).

La dispersione scolastica è un fenomeno di lunghissima durata: caratterizza particolarmente la classe d'inizio di ogni ciclo ed è "leggibile" come forma di resistenza al processo di "scolarizzazione" e/o di una certa "scrematura" iniziale dell'utenza (secondo alcune analisi diacroniche costituirebbe la risposta alla scolarizzazione di massa). Visto che si tratta di un fenomeno complesso occorre intervenirvi su più livelli.

Da alcuni anni, le azioni di ricerca sulla dispersione del Gruppo di Ricerca di Area Educativa ${ }^{4}$, pur con differenti accenti, seguono tre direttrici principali:

- il tentativo di una quantificazione precisa, almeno ai livelli locali, del fenomeno (si veda il paragrafo successivo) e la diffusione di una cultura della prevenzione;

- la ricerca dei significati attribuiti alla dispersione da parte di tutti gli attori principali in gioco (studenti a rischio e studenti eccellenti, dropout, insegnanti, dirigenti, genitori) (Bartolucci e Batini, 2016; Batini e Benvenuto, 2016; Batini e Bartolucci, 2016a; Batini, 2014);

${ }^{4} \mathrm{Ci}$ si riferisce al gruppo di Ricerca di Area Educativa "Scienze Pedagogiche e Didattiche", coordinato da chi scrive, presso il Dipartimento FISSUF dell'Università degli Studi di Perugia e, in particolare, alle ricerche legate alla cattedra di Pedagogia Sperimentale. 
- la definizione di protocolli di intervento didattico (e non solo) capaci di portare al successo formativo, rilevato con strumenti interni ed esterni al contesto di istruzione in cui ci si trova, e quindi, confrontandosi con la ricerca evidence based sul tema, offrire percorsi open access ripetibili

(Batini, Bartolucci e De Carlo, 2017; Batini e Evangelista, 2017; Batini, Cini e Paolini, 2016; Batini et al., 2016).

Nella terza direttrice, ovviamente, trova ampio spazio la lettura e lo studio degli effetti che interventi mediati dalla lettura ad alta voce o dalla lettura integrata ad altri interventi possano determinare all'interno di un contesto "reale" come il sistema di istruzione.

\section{Di che cosa parliamo quando parliamo di "dispersione scolastica"?}

Come è stato chiarito poco sopra, la dispersione scolastica, anche fermandosi alla definizione più stringente, riguarda almeno gli abbandoni, le non frequenze e le ripetenze. Ultimamente, tuttavia, in una terminologia già non priva di ambiguità, si sono innestate scelte politiche che lasciano perplessi. Sono due le problematiche principali: l'utilizzo del termine complessivo, dispersione scolastica, per indicare il solo abbandono formalizzato e l'utilizzo di costrutti relativi a fasce di età successive al "periodo critico" per individuarne la consistenza, con l'esito tragico di "contare i morti" anziché tentare di prevenire il morbo e ridurre la mortalità.

\subsection{Ragazzi fantasma?}

Il 17 novembre 2017 esce il rapporto MIUR (Miur, 2017) che, già nella nota di presentazione, si autodefinisce come un proseguimento, migliorativo, del Focus sulla dispersione del 2013: «Il presente lavoro costituisce l'aggiornamento del focus sulla dispersione scolastica pubblicato nel 2013 con dati relativi all'anno scolastico 2011/2012. La base informativa utilizzata in questa sede, come verrà illustrato nei prossimi paragrafi, è più completa e dettagliata.» (Miur, 2017, p. 3).

La funzione dell'intero processo che ha portato alla costruzione dell' Anagrafe Nazionale viene fatta risalire a «quello di costituire uno strumento di monitoraggio e, di conseguenza, di fornire strumenti per il contrasto alla dispersione scolastica; è per questo che le istituzioni scolastiche statali e pari- 
tarie sono tenute ad aggiornare in tempo reale la frequenza scolastica, comunicando l'eventuale interruzione della frequenza di ogni singolo alunno o il trasferimento ad altra scuola.» (Miur, 2017, p. 7; Miur, 2013) . $^{5}$.

La dispersione viene definita così nel rapporto: «consideriamo la dispersione come il fenomeno di allontanamento dal sistema scolastico e formativo da parte degli studenti» (Miur, 2017, p. 3).

Questa definizione, generica perché non chiarisce che cosa si intende con "allontanamento", parrebbe comunque comprendere almeno le non frequenze, tuttavia esse non si esauriscono in coloro che abbandonano tra un anno e l'altro, ma sono spesso rappresentati dalle ripetenze (che si tradurranno spesso, a loro volta, in una frequenza discontinua ma mai formalizzata).

Si ribadisce quindi come la dispersione venga analizzata, in questo rapporto, con maggiore completezza di dati rispetto al Focus che lo ha preceduto (Miur, 2013): «dopo quattro anni, l'Anagrafe è uno strumento affidabile ed è possibile arricchire lo studio del fenomeno della dispersione considerando non solo l'abbandono nel corso dell'anno scolastico ma anche quello che avviene tra un anno e l'altro, sia all'interno dello stesso ordine di scuola sia nel passaggio tra cicli scolastici, utilizzando un nuovo e più completo approccio.» (Miur, 2017, p. 10). In realtà l'attenzione del rapporto si concentra molto nel definire le varie tipologie di interruzione di frequenza ${ }^{6}$.

${ }^{5}$ Si ricorda che l'iscrizione agli anni successivi al primo è automatica.

${ }^{6}$ Il rapporto delinea cinque tipologie di interruzione di frequenza: «È possibile quindi considerare cinque "tasselli della dispersione", che congiuntamente formano l'insieme di alunni che escono dal sistema scolastico nazionale (tavola B). Essi sono rappresentati, nel dettaglio, da:

1. alunni che frequentano la scuola secondaria di I grado e che interrompono la frequenza senza valida motivazione prima del termine dell'anno (abbandono in corso d'anno);

2. alunni che hanno frequentato l'intero anno scolastico (I e II anno di corso della scuola secondaria di I grado) e che non passano nell'anno successivo né al II o III anno in regola, né al I e II anno come ripetenti, e non passano alla scuola secondaria di II grado (abbandono tra un anno e il successivo);

3. alunni che hanno frequentato l'intero anno scolastico (III anno di corso della scuola secondaria di I grado) e che non passano nell'anno scolastico successivo alla scuola secondaria di II grado né frequentano nuovamente la scuola secondaria di I grado come ripetenti del III anno di corso (abbandono tra un anno e il successivo nel passaggio tra cicli scolastici);

4. alunni che frequentano la scuola secondaria di II grado e che interrompono la frequenza senza valida motivazione prima del termine dell'anno (abbandono in corso d'anno);

5. alunni che hanno frequentato l'intero anno scolastico (dal I al IV anno di corso della scuola secondaria di II grado), che non passano nell'anno successivo né al II, III, IV, V anno in regola, né al I, II, III e IV anno come ripetenti (abbandono tra un anno e il successivo).» (Miur, 2017, p. 11). 
Non viene spiegato, tuttavia, quali indicatori siano forniti alle scuole per l'intervento tempestivo, "in tempo reale" al quale sono chiamate. Come si definisce l'interruzione di frequenza in corso d'anno? Quante sono le assenze consecutive o le assenze in un mese perché si possa parlare di interruzione di frequenza e quindi di "rischio abbandono"? Dove vengono conteggiati i ragazzi che frequentano saltuariamente? Dove vengono conteggiati coloro che incorrono in una ripetenza? La ripetenza, com'è noto (Alivernini e Lucidi, 2011; Batini e Bartolucci, 2016a) è un predittore dell'abbandono e non tenere sotto controllo le ripetenze costituisce un vulnus gravissimo alle possibilità di reale monitoraggio della dispersione ${ }^{7}$. Le non frequenze, gli abbandoni di fatto mai formalizzati, riguardano, spesso, le situazioni più complesse e il rischio che una parte consistente di questi si perda nella regolare reiscrizione all'anno successivo (che corrisponde a una volontà o a un automatismo?) è molto alto.

La maggiore accuratezza oggi possibile nel presentare i dati, grazie al funzionamento dell'Anagrafe Nazionale, consente senza dubbio l'individuazione di alcune problematiche: una su tutte la percentuale superiore all $180 \%$ (Miur, 2017, p. 34) di abbandono per i ragazzi di cittadinanza non italiana nati all'estero. Questa spaventosa percentuale pone un reale problema di inclusione e di diritto all'apprendimento da garantire ai minori, tuttavia i problemi individuati ne nascondono altri, alcuni richiamati poco sopra.

L'utilizzo sinonimico di dispersione e abbandono, quasi che la prima si esaurisse nel secondo, è dunque un problema. Un problema non soltanto nominalistico. Che accade ai ragazzi che non vengono osservati in alcun modo e non vengono contati in nessuna statistica?

Quasi dieci anni prima il MIUR aveva chiarito di aver presenti due modalità per conteggiare la dispersione (Miur, 2008): una molto simile a quella qui proposta e una relativa agli indicatori europei (per i quali si fa riferimento

\footnotetext{
${ }^{7}$ Non sarà un caso che anche all'interno delle proprie modalità di conteggiare la dispersione lo stesso rapporto riconosca come, già per la secondaria di primo grado sia possibile osservare: «La percentuale di alunni che hanno abbandonato il sistema scolastico è pari al $5,1 \%$ per gli alunni in ritardo, e allo $0,4 \%$ per gli alunni in regola. Da notare come anche gli alunni anticipatari presentino una percentuale di abbandono superiore a quella degli alunni in regola $(1,1 \%)$. La distribuzione per fasce di età mostra come l'abbandono sia più elevato per gli alunni che hanno età superiore a quella dell'obbligo scolastico, ossia superiore ai 16 anni. Nel dettaglio, dei frequentanti a settembre 2015 ha abbandonato la scuola secondaria di I grado, nel corso dell'a.s. 2015/2016 e nel passaggio all'a.s. 2016/2017, il 40,9\% degli alunni con età superiore ai 16 anni.» (Miur, 2017, p. 21). Risulta evidente come la probabilità di abbandono per i ripetenti sarebbe dunque molto più alta, mentre per i pluri-ripetenti diventa quasi una possibilità su due. Analoghi sono i rapporti nella secondaria di primo grado.
} 
al paragrafo successivo $)^{8}$. E proponeva pertanto: «Per quanto riguarda, invece, la chiave di lettura nazionale, sopra esposta, essa dimostra che l'entità degli abbandoni scolastici è rappresentata dal numero degli studenti che durante un determinato anno scolastico interrompono gli studi senza comunicarlo alla scuola. Dalle rilevazioni sugli esiti degli scrutini effettuate dal Ministero, è possibile individuare alla fine di ogni anno scolastico uno scarto, più o meno rilevante, tra il dato iniziale degli alunni iscritti e quello relativo agli alunni che di fatto al termine dell'anno risultano scrutinati. [...] In una definizione del concetto di dispersione che comprenda anche i fenomeni di irregolarità e di insuccesso scolastico, gli indicatori che delineano l'area di esposizione al fenomeno sono il tasso di ripetenza e ritardo, tasso di non ammissione e di ammissione con debito formativo.» (Miur, 2008, pp. 3-5) ${ }^{9}$.

Il diritto all'apprendimento, che viene violato anche per chi, uscito dal sistema di istruzione si rivolge alla formazione professionale ${ }^{10}$, in questo caso non può applicarsi, perché, semplicemente, questi ragazzi non esistono.

La necessità di dimensionare in modo serio il problema della dispersione scolastica, di definirne i confini e conoscere le percezioni degli attori in gioco

\footnotetext{
${ }^{8}$ «Richiamando la definizione già data nel precedente notiziario, si può affermare che esistono due dimensioni di analisi del fenomeno della dispersione scolastica. La prima è quella che fa coincidere il fenomeno della dispersione scolastica con il numero di drop-outs rilevati nel corso di un anno scolastico. Tenuto conto della complessità di variabili, sia di natura scolastica, sia relative al contesto socio-economico che influiscono sull'abbandono degli studi, si è ritenuto opportuno, in base alle informazioni disponibili di fonte ministeriale, porre l'accento su indicatori di possibile causalità del fenomeno quali:

- numero di ripetenti;

- promossi con debito;

- ritardi accumulati nei vari anni di corso;

- passaggi ad altro indirizzo.

La seconda, invece, utilizza una chiave di lettura europea che analizza il fenomeno dell'abbandono in base all'indicatore degli early school leavers, costruito utilizzando l'Indagine "Forze di Lavoro" dell'Istat. Tale indicatore fa riferimento alla quota dei giovani dai 18 ai 24 anni d'età che posseggono la sola licenza media e sono fuori dal sistema di istruzione-formazione.» (Miur, 2008, p. 2). A sua volta questa definizione, la prima, richiederebbe di definire il concetto di drop-outs, tuttavia l'elencazione degli indicatori dei quali tener conto consente di ipotizzare una visione maggiormente realistica della dispersione scolastica.

${ }^{9}$ Correttamente infatti poco oltre si stimava la dispersione a partire da un dato forte, ovvero dal dato relativo alla percentuale di successo formativo: "Andando a considerare l'età degli iscritti per ciascun anno di corso risulta infatti che la quota degli studenti regolari diminuisce dal primo al quinto anno per effetto del numero di anni di ripetenza che si sommano nel corso degli studi. Alla fine del percorso della scuola secondaria il 72,7\% degli studenti conclude gli studi senza aver incontrato ostacoli.» (Miur, 2008, p. 5).

${ }^{10}$ Lasciandogli pochissime o nessuna opzione di scelta a causa dell'attivazione di corsi relativi solo ad alcuni dei già pochi profili previsti negli accordi Stato-Regione e con l'aggravante del possibile riempimento del profilo prescelto.
} 
è rilevante, riguarda moltissimi destini individuali, ma ha un impatto notevole sull'intera società. Poco si conciliano, infatti, letture diacroniche ottimistiche con altre modalità di conteggio della dispersione scolastica (Batini e Bartolucci, 2016a; Batini, 2014).

\subsection{Ex post}

Per circoscrivere un fenomeno occorre conoscerlo, per intervenirvi affinché non si verifichi occorre monitorarlo in modo continuo e verificare gli effetti delle varie tipologie di intervento. Per intervenire in modo efficace sulla dispersione scolastica occorre che si diffonda la conoscenza del fenomeno e la gravità delle conseguenze che determina ${ }^{11}$. Occorre che le denominazioni utilizzate siano le medesime e, laddove possibile, che si intervenga prima che il fenomeno si sia manifestato.

Negli ultimi anni l'Italia utilizza sempre più costrutti che fanno riferimento alla condizione di chi:

- compreso tra 18 e 24 anni non ha raggiunto alcun titolo successivo alla secondaria di primo grado: gli early school leavers (ELS) ${ }^{12}$;

- compreso tra 18 e 24 anni d'età possiede al più il titolo di scuola secondaria di I grado o una qualifica di durata non superiore ai 2 anni e non è più in formazione: gli early leaving from education and training (ELET).

Si tratta, ovviamente, di costrutti che incrociano la dispersione dopo che è avvenuta, ponendoci dunque di fronte a un duplice problema: non permettono interventi preventivi (per l'età anagrafica dei soggetti presi in considerazione) e non sono in grado di fotografare il prodursi della dispersione (e

${ }^{11}$ Le conseguenze di un elevato tasso di dispersione scolastica possono essere, nel medio periodo, tragiche in termini di costi individuali, sociali, familiari e comunitari (Save the Children, 2017). I giovani che hanno abbandonato prematuramente l'istruzione e la formazione incontrano maggiori difficoltà nel mercato del lavoro. Il tasso di disoccupazione per i giovani tra i 25 e i 34 anni che non hanno completato l'istruzione secondaria superiore, tra i paesi OECD, è circa del 17\%, quasi il doppio rispetto al $9 \%$ di chi, invece, ha portato a termine un percorso di istruzione secondaria di secondo livello (OECD, 2017).

${ }^{12} \mathrm{Nel}$ Focus sulla dispersione scolastica (Miur, 2013), in una nota già si osservava quanto qui argomentato: «L'indicatore degli early school leavers, relativo a tutte le persone che abbandonano precocemente la scuola e che non sono incluse in programmi di formazione, consiste nella quota di popolazione di età 18-24 anni con titolo di studio non più alto dell'istruzione secondaria inferiore e non inseriti in programmi di formazione. Tale indicatore, fotografando una situazione riferita ad epoche pregresse, non consente una misura del fenomeno allo stato attuale.» (Miur, 2013, p. 5) (La sottolineatura è di chi scrive). 
quindi comprenderne meglio le dinamiche e la collocazione dei diversi possibili interventi). Nemmeno gli interventi curativi sono, a partire da quei costrutti, interventi tempestivi.

Se lo scopo che ci poniamo è quello di ridurre la dispersione scolastica in modo sensibile occorre quindi avere un monitoraggio costante e attendibile della stessa. I costrutti come quello degli ESL, degli ELET (o degli ELVET) servono per il controllo di medio periodo, non certo per una fotografia attendibile del presente. Se voglio comprendere, infatti, ciò che sta avvenendo all'interno del sistema di istruzione non posso riferirmi a soggetti che ne sono fuori, in molti casi, già da diversi anni.

Un'osservazione ex post, insomma, non sembra poter aiutare. Occorre dunque accordarsi sulla modalità più corretta per misurare la dispersione senza dimenticare che si deve trattare della modalità più adeguata a intervenirvi, tempestivamente, per prevenirla.

\section{La lettura e i suoi benefici}

Nelle ricerche che abbiamo realizzato in questi ultimi anni abbiamo potuto osservare come un training di lettura ad alta voce (solitamente articolato in circa quaranta incontri di lettura, di durata variabile ma tendenti a un'ora), inserito nel quotidiano contesto scolastico dei diversi ordini e gradi, integrato o meno da altri interventi, sia in grado di produrre risultati significativi afferenti a diversi domini e dunque cooperi, fattivamente, al raggiungimento del successo formativo.

\subsection{Interventi realizzati attraverso la sola lettura ad alta voce}

Alla scuola dell'infanzia abbiamo potuto osservare, in più sperimentazioni (cfr. fig. 1 per un esempio), realizzate nel corso degli ultimi tre anni, la capacità di un training di lettura ad alta voce di produrre risultati statisticamente significativi misurati attraverso il TEC - Test of Emotion Comprehension (Pons e Harris, 2000) - che si inserisce nel filone di ricerca di quello che spesso viene definito "emotional perspective-taking", ovvero la capacità di assumere e riconoscere le prospettive emotive degli altri. 


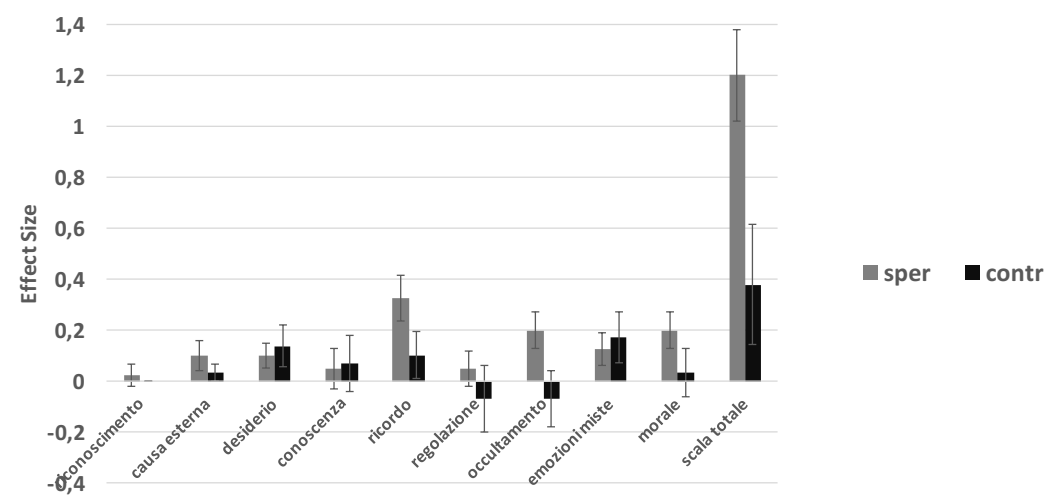

Fig. 1 - Confronto della media degli incrementi al TEC test per gruppo sperimentale e gruppo di controllo, scuola dell'infanzia

In particolare si segnala come ricorra, negli incrementi dei gruppi sperimentali rispetto ai gruppi di controllo, la significatività statistica delle scale relative alla comprensione della differenza tra emozione apparente ed emozione provata e quella relativa alla comprensione dell'influenza dei ricordi, oltre alla scala totale (come nel grafico di una delle sperimentazioni realizzate in Umbria, qui riprodotto in fig. 1), che è quella che risulta maggiormente interessante.

Analogamente si può osservare, nella scuola primaria, come le dimensioni che vengono sollecitate, rilevate attraverso lo stesso strumento, siano anche maggiori proprio in virtù del progressivo sviluppo di alcuni strumenti cognitivi ed emotivi per i quali il training di lettura ha svolto, senza dubbio, una funzione facilitante.

Se prendiamo in esame, ad esempio, i risultati di una classe terza della primaria possiamo osservare risultati di estremo interesse: le scale che raggiungono significatività statistica sono, nell'attribuzione delle motivazioni:

- dimensione III (comprensione del ruolo dei desideri);

- dimensione IV (comprensione del ruolo delle credenze);

- dimensione V (comprensione dell'influenza dei ricordi);

- dimensione VII (distinzione emozione apparente/provata);

- dimensione VIII (categorizzazione di emozioni miste);

- dimensione IX (comprensione del ruolo della morale); 
- scala totale.

Il TEC, infatti, ha proprio lo scopo di essere uno strumento in grado di valutare 1 'insieme delle componenti della comprensione delle emozioni ${ }^{13}$. La comprensione delle emozioni proprie e altrui rappresenta un pre-requisito fondamentale per una positiva socializzazione nel contesto scolastico e per una autoregolazione nella complessa serie di relazioni che si instaurano nell'ambiente scolastico e riveste quindi interesse per la prevenzione della dispersione.

Il clima di classe e le interazioni tra insegnante e studente hanno, infatti, un effetto significativo sia sull'apprendimento e sul rendimento scolastico che sull'impegno degli studenti (Pierce 1994; Bennacer, 2000; Fredriksen e Rhodes, 2004; Englund, Egeland e Collins, 2008; Vellos e Vadeboncoeur, 2015). D'altro lato, un contesto classe negativo con insegnanti punitivi contribuisce ad aumentare il rischio di abbandono (Franklin e Streeter, 1995; Reyes e Jason, 1991; Fortin et al., 2006; Nairz-Wirth e Feldmann, 2017).

Se invece proseguiamo l'osservazione alla scuola dell'infanzia e ci soffermiamo sul Test di Riordino di Storie Figurate (una sottoscala della WISCR) possiamo rubricare un'altra area di effetto della lettura. Il Test di Riordino delle Storie Figurate è un test che misura la capacità di analisi di dati narrativi visivi e di pianificazione sequenziale e, in questo caso, risultano significativi tutti e 3 i livelli (fig. 2). Si tratta di microstorie rappresentate attraverso delle immagini che debbono essere messe in ordine. Per individuare l'ordine corretto, ovviamente, occorre, interpretando le immagini, cogliere il significato della storia.

\footnotetext{
${ }^{13}$ Una sola componente viene valutata con un compito di riconoscimento, per il resto la struttura globale della prova è sempre simile: lo sperimentatore mostra al bambino un'immagine ( 23 in totale) in cui il protagonista ha il viso lasciato in bianco, raccontando contemporaneamente la relativa storia; mostra poi quattro volti con differenti espressioni emotive, e il bambino deve rispondere indicando quella pertinente rispetto alla storia.
} 


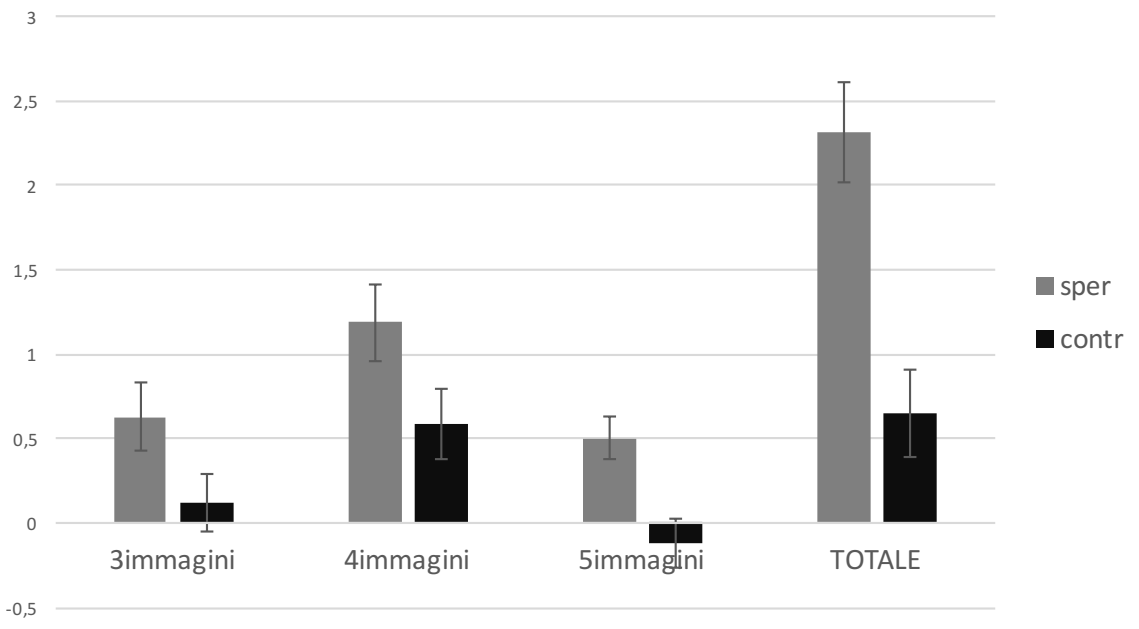

Fig. 2 - Confronto della media degli incrementi al Test di Riordino Storie figurate per gruppo sperimentale e gruppo di controllo, scuola dell'infanzia

Il test è composto da storie a 3, 4 e 5 immagini (difficoltà crescente.) Con bambini più grandi possono risultare statisticamente significative le ultime due tipologie (a 4 e 5 immagini) o l'ultima (a 5) specie se le performances con 3 immagini (o con 3 e 4) erano già buone nella rilevazione iniziale. Il test intercetta abilità legate alla comprensione e alla pianificazione. Nei training svolti presso la scuola primaria, tuttavia, seppure nella maggioranza dei casi risultino statisticamente significative la scala relativa alle storie composte da cinque immagini e la scala totale, è accaduto anche che sia risultata statisticamente significativa la scala relativa alle storie a tre immagini. In questo caso le osservazioni da fare sono due: i gruppi sperimentale e di controllo partono da livelli di abilità molto bassi e il gruppo di controllo non riesce a crescere attraverso la normale esperienza scolastica. Uno degli aspetti rilevanti che ci è saltato agli occhi - nei vari training sperimentali condotti - è stato, infatti, il ruolo del gruppo di controllo che, con la stratificazione dei risultati, rileva la scarsissima capacità della didattica tradizionale di impattare sullo sviluppo di abilità e competenze che avrebbero un riflesso positivo nella scuola stessa.

Le capacità di pianificazione hanno un ruolo fondamentale nel contesto scolastico e la loro mancanza rende complessa l'organizzazione del proprio lavoro e del proprio studio comportando conseguenze sul rendimento scolastico e sul successo formativo. 
Isolando una classe, tra le tante investigate, nella scuola primaria ci troviamo di fronte a una situazione del tipo illustrato in figura 3.

\section{Classe $5^{\circ}$ Primaria}

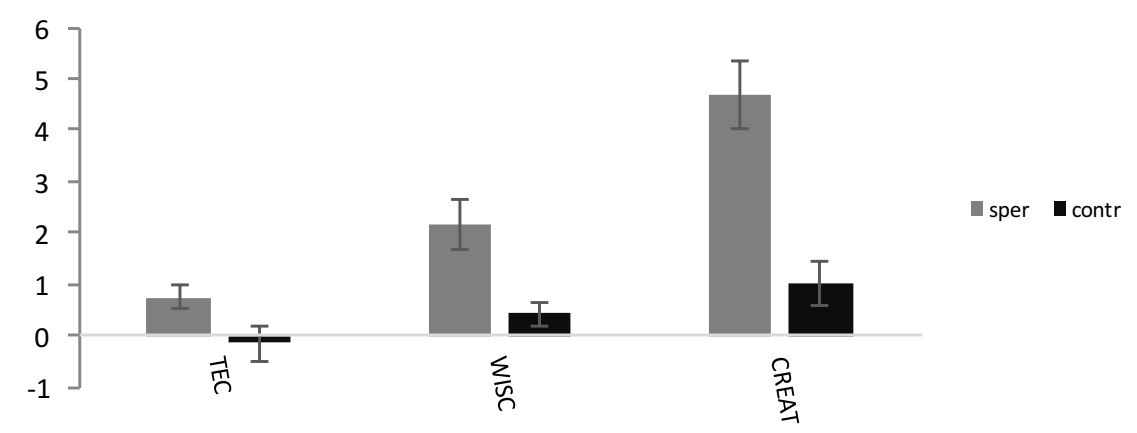

Fig. 3 - Confronto della media degli incrementi tra gruppo sperimentale e gruppo di controllo al TEC, al Test di Riordino Storie figurate, al test della creatività. Test per gruppo sperimentale e gruppo di controllo, scuola primaria (classe quinta). Tutti i 3 test qui presentati hanno dato risultati statisticamente significativi

Si è già evidenziato, commentando il grafico relativo alla scuola dell'infanzia, come i risultati raccolti mediante i primi due strumenti abbiano rilevanza sia in termini assoluti (per le abilità e competenze che si sono sviluppate nei bambini/ragazzi partecipanti) sia in relazione al successo formativo, come la letteratura ha ampiamente dimostrato (risultati analoghi si sono ottenuti con classi seconde, terze, quarte).

Per il test sulla creatività, del quale abbiamo sperimentato diverse forme $^{14}$, si tratta, invece, di una aggiunta di enorme interesse. Il test cerca di indagare la capacità di utilizzo del pensiero creativo che, pur essendo un'abilità che la scuola dichiara di promuovere è, in realtà, spesso limitata. La regola, l'applicazione della regola, la logica della risposta giusta, la logica dell'una e una sola soluzione mal si legano allo sviluppo di abilità creative.

${ }^{14}$ In una forma, della quale si trovano diversi utilizzi in letteratura, si chiede ai bambini (o al target di riferimento) di pensare, in un tempo definito, ai possibili usi di una graffetta da disegno. Nella seconda forma, invece, si presentano alcuni fogli pieni di piccoli cerchi e si chiede di farli diventare forme dotate di significato (mantenendo però il cerchio). In entrambi i casi il maggior numero di soluzioni creative si hanno quando si "sposta" il confine (posso immaginare, per esempio, che la graffetta pesi $10 \mathrm{~kg}$; posso collegare tra loro più cerchi per dare vita a un'immagine più complessa, etc.). 
In questo caso occorre dunque non soltanto coltivare e facilitare l'emersione della creatività nel percorso di istruzione, ma occorre anche fare in modo che la creatività sia apprezzata e valorizzata in contesto scolastico anziché, come a volte avviene, limitata, ingabbiata o sanzionata. Anche in questo caso i risultati tendono a replicarsi anche cambiando lo strumento e questo ci consente di ipotizzare quindi un ruolo di facilitazione del training di lettura anche sulla propensione alla creatività e sulle abilità collegate.

Concentrando l'osservazione ancora sulla scuola primaria (fig. 4) si possono, adesso, osservare i risultati ottenuti mediante un altro strumento più complesso, una batteria denominata AMOS che risulta particolarmente interessante per l'incrocio di dimensioni cognitive, metacognitive e motivazionali (Cornoldi et al., 2005).

La batteria AMOS permette, infatti, di valutare fattori metacognitivi, strategici, emotivo-motivazionali e cognitivi connessi allo studio ${ }^{15}$ ed ha mostrato di correlare in modo significativo con il rendimento scolastico e il successo formativo (Barbero Vignola e Duca, 2016).

AMOS

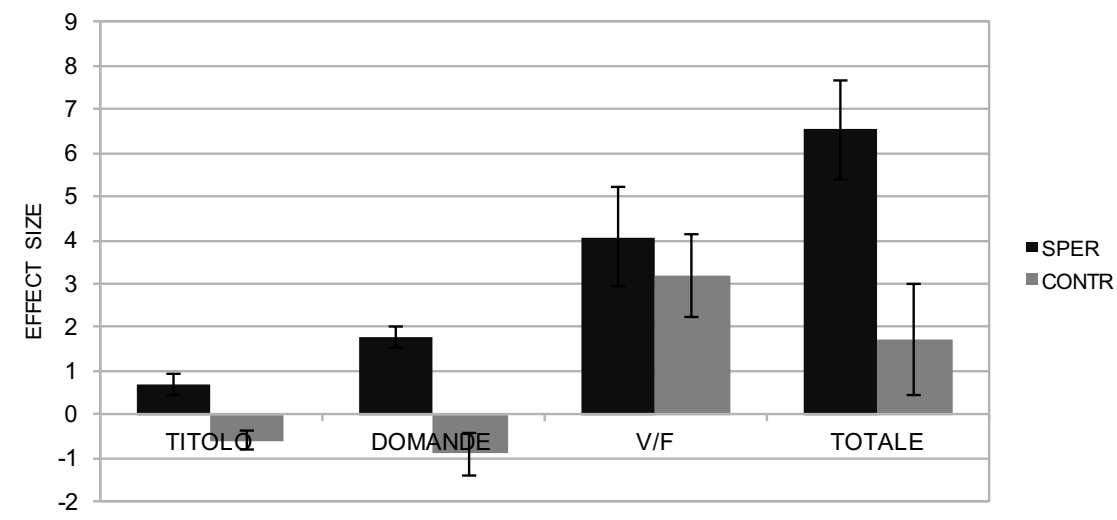

Fig. 4 - Confronto della media degli incrementi tra gruppo sperimentale e gruppo di controllo (classe quarta primaria). Risultano significative la scala relativa all'attribuzione di un titolo, la scala relativa alle domande aperte e la scala totale

${ }^{15}$ Il test AMOS 8-15 è uno strumento che valuta le abilità e le motivazioni allo studio degli studenti dagli 8 ai 15 anni (Cornoldi et al., 2005). La batteria permette di valutare fattori metacognitivi, strategici, emotivo-motivazionali e cognitivi, attraverso quattro strumenti: Questionario sull'approccio allo studio (QAS); Questionario su utilità e uso delle strategie di studio (QS1 e QS2); Questionario su convinzioni (QC1I, QC2F, QC30) e attribuzioni (QA); Prova di studio (PS). 
Le strategie di apprendimento e di autoapprendimento, d'altronde, hanno già mostrato la loro rilevanza nel determinare il successo formativo (Yusuf, 2011; Komarraju e Nadler, 2013; Batini, 2014; Fan e Wolters, 2014) così come è noto il ruolo della motivazione e delle attese degli studenti (Weber et al., 2013).

Oltre alle dimensioni motivazionali e metacognitive l'AMOS, attraverso la prova di studio, è in grado di identificare anche competenze specifiche legate alla comprensione dei testi e, nell'esempio proposto, la conferma si trova con il risultato della classe sperimentale, statisticamente significativo se comparato a quello della classe di controllo (in relazione agli incrementi medi dei due gruppi), nella somministrazione di prove rilasciate dall'Invalsi (e con altre tipologie di prove) $)^{16}$.

Di estremo interesse risultano anche gli esiti del SSQ test (Social Stories Questionnaire), uno strumento che misura la capacità empatica di un soggetto. Vengono presentati 10 racconti brevi in cui il partecipante deve identificare le espressioni che potrebbero causare turbamenti ${ }^{17}$. I partecipanti, appartenenti a una classe quarta della scuola primaria, hanno mostrato una differenza statisticamente significativa nel confronto tra gruppi (sperimentale e di controllo) prima e dopo il test (fig. 5).

${ }^{16}$ Per i test Invalsi sono stati utilizzati i fascicoli di Italiano per le classi 5 degli anni 2014/2015 e 2015/2016. Nella prima somministrazione la media dei punteggi del gruppo sperimentale è risultata di 0,49 (d.s 0,23 ), per il gruppo di controllo, invece, la media è stata pari a 0,60 (d.s. 0,06). Nella seconda somministrazione le prove del gruppo sperimentale hanno raggiunto una media di 0,65 (d.s. 0,16$)$ mentre quella del gruppo di controllo è di 0,63 (d.s. $0,14)$. Come si può osservare facilmente, il gruppo di soggetti che ha partecipato al training di lettura ad alta voce ha ottenuto un incremento medio notevole (maggiore di quello del gruppo di controllo in tutte le parti del test). Questa differenza, tuttavia, raggiunge significatività statistica solo considerando il test nel suo complesso $(F=5,619 ; \operatorname{Sign}=0,027)$. Anche i risultati di prove di controllo condotte mediante le prove PIRS si sono rivelati ottimi con il gruppo sperimentale che produce un risultato significativamente migliore rispetto al gruppo di controllo che rimane, sostanzialmente, fermo.

${ }^{17}$ Si tratta di un questionario formulato dall'Università di Cambridge (Lawson, BaronCohen e Wheelwright, 2004) che va ad indagare le capacità di empatia dell'individuo. Tale questionario presenta 10 racconti brevi in cui il partecipante deve identificare le espressioni che potrebbero causare turbamenti. Il miglioramento statisticamente significativo, rilevato nel gruppo sperimentale rispetto al gruppo di controllo per quanto riguarda il SSQ test, potrebbe essere dovuto al fatto che il gruppo sperimentale, beneficiando del training di lettura ad alta voce, si è confrontato con testi che hanno consentito partecipazione e immedesimazione nei vari personaggi e nelle situazioni che questi ultimi vivevano e dunque ha "allenato" la capacità di assumere il punto di vista altrui (o anche più punti di vista rispetto alla stessa situazione). 


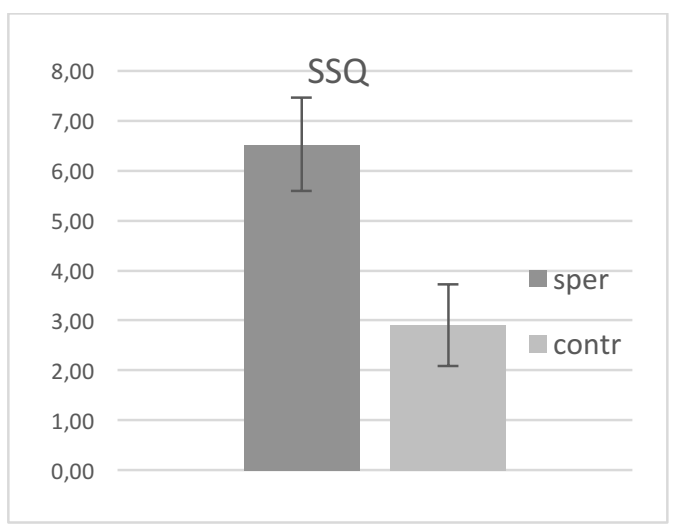

Fig. 5 - Confronto della media degli incrementi tra gruppo sperimentale e gruppo di controllo per SSQ test. Scala totale (la scala risulta statisticamente significativa)

La comprensione di ciò che potrebbe causare turbamento agli altri risulta un predittore piuttosto importante dello sviluppo di competenze empatiche e riguarda quindi un fattore fondamentale per la socializzazione con i pari che costituisce uno degli obiettivi fondamentali dell'esperienza di istruzione (ma anche per la comprensione reciproca con gli adulti che insistono nel sistema scolastico).

Spostando lo sguardo sulla scuola secondaria di primo grado possiamo osservare con interesse i risultati di una batteria complessa come il CAS (Cognitive Cognitive Assessment System).

Il CAS si fonda sulla teoria PASS di A.R. Lurija, che considera il funzionamento cognitivo basato su quattro processi essenziali: Pianificazione; Attenzione; Simultaneità; Successione. Studi in letteratura hanno ampliamente dimostrato la correlazione fra performances a questo test e successo scolastico (Naglieri e Rojahn, 2004; Naglieri et al., 2006).

In un esperimento realizzato con una classe seconda di una secondaria di primo grado (fig. 6) possiamo evidenziare come il gruppo sperimentale riveli una tendenza all'incremento superiore al gruppo di controllo in tutte le scale e come nella scala totale questa differenza di incremento diventi statisticamente significativa. 


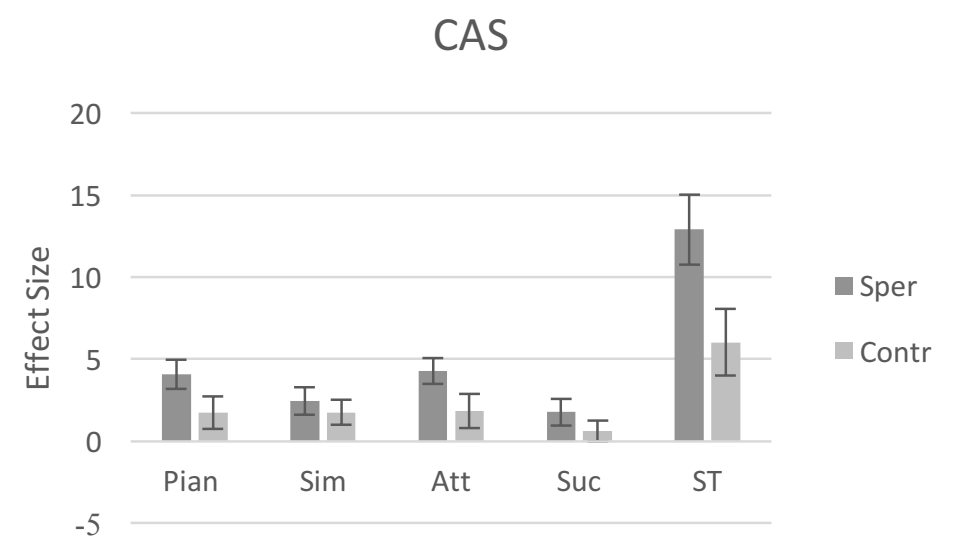

Fig. 6 - Confronto della media degli incrementi tra gruppo sperimentale e gruppo di controllo per batteria CAS (classe seconda, scuola secondaria di primo grado). La scala totale risulta statisticamente significativa

La batteria CAS ha, come già specificato, una correlazione forte con il successo formativo e testimonia una capacità della lettura di agire anche su dimensioni spiccatamente cognitive.

\subsection{Interventi integrati con la lettura ad alta voce}

Oltre agli interventi attraverso training di lettura, le sperimentazioni di questi anni, in particolar modo con un progetto pluriennale di prevenzione della dispersione denominato NoOut ${ }^{18}$ (Batini, 2018; Batini, Bartolucci e De Carlo, 2017; Batini e Evangelista, 2017; Batini e Bartolucci, 2016a; Batini, Cini e Paolini, 2016; Batini et al.,2016), hanno consentito di osservare gli effetti della lettura integrata con altri strumenti, in particolare con l'approccio dell'orientamento narrativo (Batini, 2015) e con una didattica attiva e partecipativa (impreziosita dall'utilizzo di compiti di realtà e di giochi non aleatori) mirata al recupero e allo sviluppo delle competenze di base.

${ }^{18}$ Il progetto è stato finanziato nel 2015-2017 dalla Fondazione CR di Firenze con il partenariato dell'Università di Perugia, dell'ISFOL, e delle Associazioni Pratika e Nausika (Arezzo). Il progetto nasce con l'intento di prevenire la dispersione scolastica in due territori toscani (provincia di Arezzo e provincia di Firenze) per tutti i gradi scolastici e di modellizzare gli interventi in modo da costruire percorsi e strumenti replicabili e utilizzabili dagli insegnanti per prevenire la dispersione. I materiali sono, infatti, rilasciati in modalità open access. Si veda a questo proposito www.dispersione.it. 
Si evidenziano, allora, in NoOut e in altre sperimentazioni comunque caratterizzate da un approccio integrato (orientamento narrativo, recupero competenze di base, lettura ad alta voce), risultati simili a quelli già commentati nel paragrafo precedente, con alcune differenze interessanti riguardo ai due strumenti più complessi: AMOS e CAS. Per quello che riguarda il test AMOS è interessante notare come gli approcci nei quali la lettura risulta integrata con gli altri due interventi (è bene ricordare che si trattava di interventi della durata massima di 70 ore complessive, più spesso di durata minore, svolti in orario curricolare) producano miglioramenti significativi anche nei questionari relativi all'approccio allo studio e alle strategie di studio.

Fondamentali risultano gli esiti della batteria CAS che, utilizzata in tutte le scuole coinvolte, dalla primaria sino ai ragazzi dropout, già fuoriusciti dal sistema di istruzione (e inseriti in percorsi per l'acquisizione di una qualifica professionale per assolvere al diritto dovere all'istruzione/formazione), ha mostrato non soltanto effetti statisticamente significativi in tutti i gradi (e nelle diverse aree geografiche in cui il progetto ha svolto le proprie attività) ma consente alcune osservazioni ulteriori:

- nella scuola primaria oltre alla scala totale risultano significative tutte le sottoscale, mentre i gruppi di controllo tendono a decrescere. Questo rilievo determina un punto interrogativo circa la relazione tra funzioni cognitive e didattica tradizionale;

- nella scuola secondaria di primo grado oltre alla scala totale risultano significative le sottoscale relative a attenzione, pianificazione, successione;

- nella scuola secondaria di secondo grado oltre alla scala totale risultano significative le sottoscale relative a pianificazione e successione;

- con i ragazzi dropout oltre alla scala totale risulta significativa la sottoscala relativa a simultaneità.

Risulta evidente come si possa determinare, a parità di condizioni, una maggiore facilità di intervento nei gradi iniziali. Interventi mediati dalla lettura ad alta voce o interventi integrati tra orientamento narrativo, didattiche attive e lettura sono dunque utili per tutte le fasce di età e sono in grado di produrre effetti visibili in tempi decisamente brevi, tuttavia se utilizzati in un'età infantile o prossima all'infanzia dispiegano al massimo la propria potenza (e possono contribuire maggiormente a una logica di prevenzione anziché di cura).

La lettura ad alta voce, utilizzata in modo semplice e quotidiano e la lettura integrata con altri strumenti possono costituire un potente antidoto alla dispersione scolastica, specie se utilizzati con continuità. 


\section{Conclusioni: verso una proposta}

In questi dieci anni abbiamo avuto l'opportunità di osservare molteplici effetti della lettura in contesti diversi e con soggetti di differenti condizioni ed età anagrafiche, sino ad arrivare a soggetti molto anziani, affetti da malattie dementigene (Batini, Toti e Bartolucci, 2016).

Negli stessi anni la nostra lente sulla dispersione scolastica, assieme agli sforzi per definirne i confini e le dimensioni e a rifuggire dalle semplificazioni e da scelte di misurazione insoddisfacenti, ha cercato di sollevare l'attenzione su un fenomeno che è lecito considerare un'emergenza sociale. L'intenzione che ci ha mosso è stata quella di provare a intervenirvi con varie modalità, tutte orientate alla promozione dello studente e dei suoi apprendimenti, in una logica complessiva di empowerment per tentare di definire percorsi, basati sulle evidenze e sull'ascolto di tutte le voci (Bartolucci e Batini, 2016; Batini, 2014).

L'interesse specifico per gli effetti di determinate scelte didattiche nei confronti di quei soggetti che incontrano maggiori difficoltà nel sistema di istruzione ${ }^{19}$ ci ha convinto, a seguito di esiti incoraggianti, dell'importanza dell'attivazione mirata al recupero di competenze di base o al loro potenziamento (Hattie, 2009; 2012). L'integrazione dell'orientamento costituisce un ulteriore antidoto alla dispersione (Batini, 2014). Laddove si struttura una collaborazione positiva con gli insegnanti, percorsi integrati come quelli qui sinteticamente presentati ${ }^{20}$, riescono a compiere veri e propri "miracoli" didattici risvegliando motivazioni, attivando risorse, risignificando l'esperienza scolastica, trasformando uno svantaggio in un percorso da fare, anziché in una montagna invalicabile.

Crediamo dunque di poter proporre, approfittando di una particolare congiuntura, che vede, in Italia, il lancio del percorso 0-6, sempre meno pensato in termini di semplice accudimento e sempre più in termini educativi e formativi, un itinerario possibile per una riduzione sistemica della dispersione che sarebbe interessante mettere alla prova su un territorio più vasto.

\footnotetext{
${ }^{19}$ Ricordando che ci situiamo in una situazione che si rivela di doppio svantaggio: negli istituti con un indice socio-economico-culturale più basso, infatti, più di 1 quindicenne su 4 (il $27,4 \%$ ) è ripetente, mentre negli istituti con indice alto la quota scende quasi a 1 su 23 (il $4,4 \%$ ). Uno studente di quindici anni su 2 (il 47\%) proveniente da un contesto svantaggiato, inoltre, non raggiunge il livello minimo di competenza in lettura, otto volte tanto rispetto a un coetaneo cresciuto in una famiglia agiata (Save The Children, 2017).

${ }^{20}$ Si rimanda per l'accesso ai materiali di ricerca e didattici open access al sito dispersione.it.
} 
Si tratta di promuovere, in modo attivo, percorsi quotidiani di lettura ad alta voce sistematici e progressivi, sin dal nido, con un'intenzionalità didattica e una progettualità pedagogica che favorisca l'espressione delle proprie sensazioni, riflessioni, commenti ma che non persegua ansie da pre-scolarizzazione e valutative. La lettura ha, in sé, un potere e un potenziale enorme.

Sin dalla scuola primaria sarebbe opportuno avviare, parallelamente all'attività di lettura, che non deve essere interrotta né sottratta alla dimensione quotidiana, percorsi di didattica attiva orientata allo sviluppo e alla "prova sul campo" delle competenze di base. Le attività legate all'orientamento, inteso come percorso formativo teso prima alla conoscenza di sé, poi progressivamente al dialogo e al gioco (poi confronto, analisi) con le professioni per svilupparsi, dalla secondaria, nella riflessione sulle relazioni (con sé stessi, con gli altri, con la società e il mondo) per giungere a lavorare su quelle competenze che consentono l'autorientamento (dalla capacità di selezionare le informazioni, sino all'individuazione dei propri desideri, alla capacità di trasformarli in obiettivi e allo sviluppo di competenze progettuali $\mathrm{e}$ disposizione all'agentività, per disegnare tragitti e percorrerli).

Questa proposta triadica (lettura, didattiche attive orientata alle competenze di base, orientamento narrativo) potrebbe, a costi minori di quelli affrontati negli ultimi anni per fronteggiare la dispersione, produrre un'inversione di rotta, tentando, cioè, di rendere possibile quello che possibile deve essere (anche laddove appare impossibile): il diritto di ogni bambina e bambino, di ogni ragazza e ragazzo di avere reali opportunità di apprendimento, di poter immaginare un futuro e di poter prendere possesso degli strumenti per costruirlo.

Il guaio è che i grandi si illudono di sapere che cosa succede dentro la testa di un bambino di dieci anni. Ed è impossibile sapere di una persona che cosa pensa, se quella persona non lo dice (Ian Mc Ewan, 2002, L'inventore di sogni, Torino, Einaudi, p. 8).

Se ci piaceva leggere lei non se lo domandava. Lei ci leggeva storie, una dopo l'altra, ogni giorno, senza darne troppo conto e senza chiederci mai troppo. Prima o poi, ciascuno, trovò la storia che lo riguardava (Brano estratto da documento privato).

\section{Riferimenti bibliografici}

Alivernini F. and Lucidi F. (2011), "Relationship between social context, self-efficacy, motivation, academic achievement, and intention to drop out of high school: A longitudinal 
study", Journal of Educational Research, 104: 241-252.

Aram D. and Aviram S. (2009), "Mothers' storybook reading and kindergartners' socioemotional and literacy development", Reading Psychology, 30, 2: 175-194.

Aram D. and Shapira R. (2012), "Parent-child shared book reading and children's language, literacy, and empathy development", Rivista Italiana di Educazione Familiare, 7, 2: 5565.

Barbero Vignola G. e Duca V. (2016), "Stare bene a scuola, apprendere e crescere in modo positivo", Studi Zancan, 3: 29-38.

Bartolucci M. e Batini F. (2016), C'era una volta un pezzo di legno, FrancoAngeli, Milano.

Batini F. (2014), Dropout, Fuorionda-Altreducazione, Arezzo.

Batini F. (2015), "To Choose or to Plan? The Narrative Orientation as an Answer to the Change of Orientation Need", Pedagogia Oggi, 1.

Batini F. (2018), Prevenire l'abbandono scolastico con la didattica per competenze: un progetto evidence based, in Ulivieri S., Binanti L., Colazzo S. e Piccinno M., (a cura di), Scuola Democrazia Educazione Formare ad una nuova società della conoscenza e della solidarietà, Pensa Multimedia, Lecce-Rovato (BS).

Batini F. e Bartolucci M., a cura di (2016a), La dispersione scolastica. Ascoltare i protagonisti per comprenderla e prevenirla, FrancoAngeli, Milano.

Batini F. e Bartolucci M. (2016b), "Chi legge ragiona meglio? Abitudini di lettura e funzioni di ragionamento", Ricerche di pedagogia e didattica, 11, 3: 37-45.

Batini F., Bartolucci M. e De Carlo M.E. (2017), "Fight Dispersion Through Education: The Results of the First Cycle of the NoOut Project", Mind, Brain and Education, XI, 4: 201 212.

Batini F. e Benvenuto G. (2016), "Le parole disperse. La voce degli studenti drop-out e la ricerca etnografica in pedagogia (Student drop-out voice and ethnographic research in education)", in Szpunar G., Sposetti P. e Sanzo A., a cura di, Narrazione e educazione, Nuova Cultura, Roma.

Batini F., Cini S., Evangelista M. e Pastorelli L. (2016), Io non ho paura. Asse linguistico, Loescher, Torino.

Batini F., Cini S. e Paolini A. (2016), Io non ho paura. Asse matematico, Loescher, Torino.

Batini F. e Evangelista M. (2017), Da studenti a ricercatori. Percorso completo di didattica della ricerca, Giunti, Firenze.

Batini F., Toti G. e Bartolucci M. (2016), "Neuropsychological benefits of a narrative cognitive training program for people living with dementia: A pilot study", Dementia e Neuropsychologia, 10, 2: 127-133.

Beck I.L., McKeown M.G. and Kucan L. (2002), Bringing words to life: Robust vocabulary instruction, Guilford Press, New York, NY.

Bennacer H. (2000), "How the Socioecological Characteristics of the Classroom Affect Academic Achievement", European Journal of Psychology of Education, 15, 2: 173-189.

Bombardelli O. (2001), "Scuola e famiglia contro la dispersione scolastica" in Bombardelli O., a cura di, Educatori del terzo millennio, A.ge-Uciim, Tione di Trento.

Cambi F. (2012), "Genitori e figli attorno al libro", Rivista Italiana di Educazione Familiare, 7, 2: 23-27.

Catarsi E., a cura di (2011), Educazione alla lettura e continuità educativa, Edizioni Junior, Bergamo.

Coplan A. (2004), "Empathic engagement with narrative fictions", The Journal of aesthetics and art criticism, 62, 2: 141-152.

Cornoldi C., De Beni R., Zamperlin C. e Meneghetti, C. (2005), Amos 8-15. Abilità e motivazione allo studio: prove di valutazione per ragazzi dagli 8 ai 15 anni, Erickson, Trento. 
Dalton B., Gennie E. and Ingels S.J. (2009), Late High School Dropouts: Characteristics, Experiences, and Changes Across Cohorts (NCES 2009-307), National Center for Education Statistics, Institute of Education Sciences, US Department of Education, Washington, DC.

Duursma E., Augustyn M. and Zuckerman B. (2008), "Reading aloud to children: the evidence", Arch Dis Child, 93: 554-557.

Emili E.A. (2017), "Tecniche per la promozione della lettura nella scuola primaria", Lifelong Lifewide Learning, 12, 29: 101-113.

Englund M.M., Egeland B. and Collins W.A. (2008), "Exceptions to High School Dropout Predictions in a Low-Income Sample: Do Adults make a Difference?", Journal of Social Issues, 64, 1: 77-94.

Fan W. And Wolters C.A. (2014), "School motivation and high school dropout: The mediating role of educational expectation”, British Journal of Educational Psychology, 84, 1: 22-39.

Farrant B.M. and Zubrick S.R. (2013), "Parent-child book reading across early childhood and child vocabulary in the early school years: Findings from the Longitudinal Study of Australian Children", First Language, 33, 3: 280-293.

Fortin L., Marcotte D., Potvin P., Royer E. and Joly J. (2006), "Typology of Students at risk of Dropping out of School: Description by Personal, Family and School Factors", European Journal of Psychology of Education, 21, 4: 363-383.

Franklin C. and Streeter C.L. (1995), "Assessment of middle class youth at-risk to dropout: School, psychological and family correlates", Children and Youth Services Review, 17, 3: 433-448.

Fredriksen K. and Rhodes J. (2004), "The role of Teacher relationships in the Lives of Students", New Directions for Youth Development, 103: 45-54.

Hattie J. (2009), Visible learning: A synthesis of over 800 meta-analyses on achievement, Routledge, London.

Hattie J. (2012), Visible learning for teachers: Maximizing impact on learning, Routledge, London.

Horowitz-Kraus T., Vannest J.J. and Holland S.K. (2013), "Overlapping neural circuitry for narrative comprehension and proficient reading in children and adolescents", Neuropsychologia, 51, 13: 2651-2662.

Hutton J.S., Horowitz-Kraus T., Mendelsohn A.L., DeWitt T., Holland, S.K. and C-MIND Authorship Consortium (2015), "Home reading environment and brain activation in preschool children listening to stories", Pediatrics, 136, 3: 466-478.

Isbell R., Sobol J., Lindauer L. and Lowrance A., (2004), “The effects of storytelling and story reading on the oral language complexity and story comprehension of young children", Early Childhood Education Journal, 32, 3.

Johnston V. (2016), "Successful Read-Alouds in Today's Classroom", Kappa Delta Pi Record, 52, 1: 39-42.

Komarraju M. and Nadler D. (2013), "Self-efficacy and academic achievement: Why do implicit beliefs, goals, and effort regulation matter?", Learning and Individual Differences, 25: $67-72$.

Korat O., Shamir A. and Heibal S. (2013), "Expanding the boundaries of shared book reading: E-books and printed books in parent-child reading as support for children's language", First language, 33, 5: 504-523.

Lane H.B. and Wright T.L. (2007), "Maximizing the effectiveness of reading aloud", The Reading Teacher, 60, 7: 668-675. 
Lawson J., Baron-Cohen S. and Wheelwright S. (2004), "Empathising and systemising in adults with and without Asperger Syndrome", Journal of Autism and Developmental Disorders, 34, 3: 301-310.

Liverta Sempio O., Confalonieri E. e Scaratti G., a cura di (1999), L'abbandono scolastico. Aspetti culturali, cognitivi, affettivi, Cortina, Milano.

Mar R.A. and Oatley K. (2008), "The function of fiction is the abstraction and simulation of social experience", Perspectives on psychological science, 3, 3: 173-192.

Mata M.D.L., Monteiro V. and Peixoto F. (2012), "Attitudes towards mathematics: effects of individual, motivational, and social support factors", Child Development Research.

Matthiessen C. (2013), "The hidden benefits of reading aloud - even for older kids", Great School.

MIUR - Ufficio Statistica e Studi (2008), La dispersione scolastica, maggio 2008, Roma.

MIUR - Ufficio Statistica e Studi (2013), Focus La dispersione scolastica, giugno 2013, Roma.

MIUR - Ufficio Statistica e Studi (2017), La dispersione scolastica nell'a.s. 2015/2016 e nel passaggio all'a.s. 2016/2017, novembre 2017, Roma.

Naglieri J.A., De Lauder B.Y., Goldstein S. and Schwebech A. (2006), WISC-III and CAS: Which correlates higher with achievement for a clinical sample?, School Psychology Quarterly, 21, 1: 62-76.

Naglieri J. A. and Rojahn J. (2004), "Construct validity of the PASS Theory and CAS: correlations with achievement", Journal of Educational Psychology, 96: 174-181.

Nairz-Wirth E. and Feldmann K. (2017), “Teachers' views on the impact of teacher-student relationships on school dropout: a Bourdieusian analysis of misrecognition", Pedagogy, Culture \& Society, 25, 1: 121-136.

OECD (2009), PISA 2009 Assessment Framework. Key competencies in reading, mathematics and science, OECD Publishing, Paris.

OECD (2017), Education at a Glance 2017: OECD Indicators, OECD Publishing, Paris.

Pierce C. (1994), "Importance of Classroom Climate for at-risk Learners", The Journal of Educational Research, 88, 1: 37-42.

Pons F. and Harris P. (2000), Test of emotion comprehension: TEC. University of Oxford.

Pontecorvo C. e Pontecorvo M. (1986), Psicologia dell'educazione. Conoscere a scuola, Il Mulino, Bologna.

Reyes O. and Jason L.A. (1991), "An evaluation of a high school dropout prevention program", Journal of Community Psychology, 19, 3: 221-230.

Ronfani L., Sila A., Malgaroli G., Causa P., Manetti S., Garofolo T. and AIB A.I.B. (2006), "La promozione della lettura ad alta voce in Italia", Quaderni acp, 1.

Sabates R., Akyeampong K., Westbrook J. and Hunt F. (2010), "School Drop-out: Patterns, Causes, Changes and Policies", Background paper prepared for the Education for All Global Monitoring Report 2011.

Save the Children (2017), Futuro in partenza? L'impatto delle povertà educative in Italia, testo disponibile al sito: https://www.savethechildren.it/cosa-facciamo/pubblicazioni/futuro-partenza.

Sénéchal M. (1997), “The differential effect of storybook reading on preschoolers' acquisition of expressive and receptive vocabulary", Journal of Child language, 24, 1: 123-138.

Sénéchal M. and LeFevre J.A. (2002), "Parental involvement in the development of children's reading skill: A five-year longitudinal study", Child development, 73, 2: 445-460.

Speer N.K., Reynolds J.R., Swallow K.M. and Zacks J.M. (2009), "Reading stories activates neural representations of visual and motor experiences", Psychological science, 20, 8: 989-999.

Vellos R.E. and Vadeboncoeur J.A. (2015), "Rebuilding Attendance Practices with Youth: 
The role of Social mediation", Educational Studies, 41, 1-2: 91-108.

Weber HS., Lu L., Shi J. and Spinath F.M. (2013), "The roles of cognitive and motivational predictors in explaining school achievement in elementary school”, Learning and Individual Differences, 25: 85-92.

Yusuf M. (2011), "The impact of self-efficacy, achievement motivation, and self-regulated learning strategies on students' academic achievement", Procedia-Social and Behavioral Sciences, 15: 2623-2626. 


\section{II contributo della lettura contro la dispersione scolastica}

di Agnese Rosati

\section{Introduzione}

Il presente saggio invita ad una riflessione sul significato formativo della lettura, posto in relazione con un fenomeno, qual è quello della dispersione scolastica, che riguarda in primo luogo i soggetti coinvolti, le loro famiglie e, soprattutto, le politiche istituzionali. I dati dichiarano che in Italia come in altri paesi, il problema della dispersione costituisce un tema sul quale intervenire con una mobilitazione capace di coinvolgere il tessuto sociale, le istituzioni e le scelte politiche.

Quello che in queste pagine si intende tuttavia porre in risalto è l'aspetto formativo della lettura. Il recupero e la valorizzazione di questa dimensione, nella quale come pedagogisti, educatori e docenti crediamo, contribuisce a fare della lettura un prezioso strumento per la crescita dei singoli e della collettività.

Nelle pagine che seguono sono indicate le finalità educative della lettura, da individuare nella formazione di un pensiero libero, autonomo, immune ai pregiudizi e ai luoghi comuni, a testimonianza di una solida maturità intellettuale che, a sua volta, si esprime compiutamente nel modo di essere e di pensare sistemico degli uomini (Morin, 1999, 2001, 2015, 2016; Goleman e Senge, 2016). Libertà di pensiero, autonomia delle scelte e creatività delle idee, sono le ragioni che mobilitano e sostengono un impegno alla lettura, in onore a quella "flessibilità intellettuale" che allena le competenze logiche, emotive e sociali dei soggetti. 


\section{La promozione della lettura: un impegno condiviso}

In altri contributi e convegni si è avuto modo ed occasione di richiamare l'attenzione sulla lettura. Inseparabile e ricorrente, accanto al tema, è la dimensione progettuale, che non può restare separata per riguardare la classe $\mathrm{e}$ dunque esclusivamente gli allievi e i maestri, poiché coinvolge anche i genitori e la società intera, quel mondo extra-scolastico nel quale le persone vivono esperienze di alterità e condividono storie, narrazioni, vicissitudini e sentimenti. Sensibili alla diffusione del cosiddetto 'abito del lettore', inoltre, sono coloro che nell'ambito della lettura operano in forme diverse: autori, editori, giornalisti, pubblicisti e associazioni. Queste ultime, in particolar modo, si impegnano attivamente in un'opera di sensibilizzazione nella popolazione, per rendersi protagoniste di numerose iniziative di promozione alla lettura. Fra queste si ricordano le giornate autunnali di "Piovono libri", "Più libri più liberi", fiera nazionale della piccola e media editoria e "Libriamoci", sostenuta dal Ministero dei Beni Culturali e del Turismo in accordo con il MIUR, come le campagne nazionali di "Tempo di libri" (fiera internazionale di Milano del mese di Marzo), la "Giornata mondiale del libro e del Diritto d'Autore" (23 Aprile), il "Maggio dei libri" (dal 2011) e la "Festa del libro" (23 Maggio). Scopo di questa intensa e capillare azione di promozione, sostenuta in primo luogo dal Centro per il Libro e la Lettura (CEPELL) e dall'Associazione italiana editori (Aie), è l'incremento delle pratiche di lettura che da attività o passione di poche persone si auspica possa tradursi in una consueta abitudine, capace di alimentare un nuovo comportamento sociale, indipendentemente dall'età dei soggetti coinvolti.

Per rafforzare il buon comportamento dei piccoli e dei giovani lettori non mancano inoltre progetti ed iniziative che incoraggiano al "consumo" dei libri, contando sulla collaborazione degli adulti, come genitori, personale bibliotecario, addetti alle ludoteche, educatori ed insegnanti.

L'elemento più interessante e da sottolineare in queste esperienze è abbastanza spesso il coinvolgimento delle famiglie e della comunità. Il momento della condivisione, difatti, ben si presta ad una pratica di scambio e ad una negoziazione di significati che fa del libro uno strumento prezioso nei percorsi di cittadinanza (perché leggere è un diritto!) e nei momenti di scambio interculturale, favoriti ad esempio dai racconti di storie e di esperienze che, con una puntuale e dettagliata descrizione dei soggetti, dei contesti e dei tempi, consente ai lettori di intraprendere un viaggio esplorativo alla scoperta di realtà nuove, insolite e sconosciute, spesso distanti dal proprio mondo e dalla consueta quotidianità. La lettura avvicina le persone, promuove il dialogo e lo scambio fra culture e generazioni. Questo è quanto accade anche 
nelle occasioni formative ideate dalle istituzioni scolastiche, come ad esempio quella di Buffalo a New York. Accanto alle "gare" che collocano sul podio il lettore più appassionato (lo studente che ha letto più libri in un anno scolastico), ci sono anche spazi e momenti di ascolto, di riflessione e di confronto che uniscono istituzioni, studenti, genitori e territorio. Nella città di Buffalo, docenti e dirigente scolastico hanno pensato ad una sfida, detta appunto "La sfida dei principi", allo scopo di incentivare fra gli allievi la lettura dei testi. Si tratta di una vera e propria competizione che si conclude con il conferimento di una cintura di diverso colore. Proprio come avviene nello sport del karate, ai diversi ritmi di lettura (relativi al tempo impegnato nell'attività e al numero dei testi letti) corrisponde un colore. Al miglior lettore viene assegnata la doppia cintura nera, simbolo del record conseguito con oltre dieci mila minuti di lettura, registrati nel Lit Fit. Quella di Buffalo, in cui il Dirigente scolastico, i docenti e la stessa comunità crede molto, non rappresenta una realtà isolata, poiché le scuole statunitensi, indipendentemente dal grado e dall'età degli allievi, investono molto tempo nelle attività di lettura. In queste scuole si scommette e si investe su esperienze del genere, perché contribuiscono a definire il profilo e la "cultura" delle singole istituzioni, in quanto tale «insieme di modi di vivere accettati e caratteristici di un gruppo di persone, - con - i loro comportamenti, le loro convinzioni e verità» (Casey Carter, 2016, p. 15) possono tradursi in una cultura in grado di influire profondamente sulla formazione del carattere, così da far emergere le buone abitudini e la unicità delle persone (Cfr. ibidem). Anche molte scuole italiane, di primo e secondo ciclo, sono particolarmente attive nel sostenere la lettura, valorizzata da numerosi progetti finanziati anche dall'Unione Europea. Fra queste importanti attività si ricordano "In vitro. Un progetto sperimentale di promozione della lettura" (2016), "Read On" (2017) nell'ambito del Programma Creative Europa 2014-2020, "Open the doors to reading" nell'ambito del Programma Erasmus Plus, oltre a tante altre esperienze ed iniziative messe in atto dai comuni e dalle regioni. Il bisogno di leggere e la sollecitazione a soddisfare tale necessità, ispira molti progetti a livello internazionale, sostenuti dai ministeri nazionali, dalle fondazioni e dalle libere associazioni, come ad esempio il "National Literacy Trust" promotore dei programmi "National Young Readers", "Early Reading Connects" e "Reading For Life". Il Ministero della Cultura in Spagna ha avviato un programma, denominato "Plan de fomento de la lectura", articolato nel triennio 2017-2020, dopo il primo avvio nel 2001. Piani Nazionali, inoltre, sono stati attivati anche in Cile (2015-2020) e Messico, come in Germania, Irlanda, Norvegia e Slovacchia, dove molte interessanti iniziative coinvolgono bam- 
bini, giovani e adulti insieme. Un esempio è quello della "Semana da Leitura" (05-09 Marzo 2018) che in Portogallo rientra nella serie di iniziative volte ad animare il "Plano Nacional de Leitura", in risposta alla carente propensione alla lettura fra la popolazione che emerge dalle analisi comparative (Forum del libro, 2015, p. 11). Le ricerche pubblicate nel 2015, dichiarano che gli Svedesi sono abili lettori, mentre i Portoghesi non coltivano particolarmente questo interesse, come gli Italiani (Rapporto sullo stato dell'editoria, 2017) ${ }^{1}$, i Romeni, gli Ungheresi e i Greci. Dallo studio si evince un maggiore interesse culturale nei paesi del Nord rispetto a quelli dell'Europa occidentale e orientale, dove negli ultimi dieci anni gli Stati hanno investito nella promozione della lettura e delle iniziative culturali. Le statistiche tengono comunque conto anche della forte crisi economica che ha influito in maniera considerevole sulla spesa della popolazione destinata alle attività culturali (cinema, teatro, manifestazioni culturali e acquisto di libri). A livello locale e nazionale sono state realizzate un po' ovunque numerose esperienze ed attività di lettura, con laboratori dedicati a bambini e adulti, per incentivare l'incontro con i libri e gli autori. Nelle piazze, negli spazi espositivi aperti, come in luoghi pubblici (cinema, teatri, musei e parchi) e nelle librerie, momenti di lettura silenziosa e di scrittura creativa si alternano a riflessioni condivise e a letture ad alta voce. Il fiorire di queste apprezzabili iniziative mostra la maggiore e diffusa consapevolezza circa il fatto che un popolo che non legge non può contribuire allo sviluppo sociale ed economico del proprio paese, perché non possiede le competenze creative, ideative e imprenditoriali che permettono di sostenere il cambiamento, anche in ambito organizzativo e professionale. Chi non legge, legge raramente e male, ha pochi stimoli culturali, necessari invece per generare nuove idee ed interessi. L'identikit del "debole lettore" è quello di una persona scarsamente motivata, poco interessata a ciò che accade, generalmente apatica e indifferente al contesto sociale perché non si informa, non ha obiettivi conoscitivi né avverte bisogni di formazione personale. Leggere, allora, può rappresentare un passo iniziale per un cambiamento culturale significativo, in quanto possibilità di accesso a quei processi multidirezionali che rendono possibile una maggiore inclusione sociale. La lettura, difatti, offre gli strumenti per riflettere sulla realtà, per indagarne la natura complessa, le fitte problematiche

${ }^{1}$ In Italia, tuttavia, nei primi dieci mesi del 2017 il fatturato del settore della piccola e media editoria è cresciuto nei primi dieci mesi dell'1,5\%. Le opere e i diritti di 7.445 titoli italiani venduti all'estero sono aumentati del 13,6\% rispetto all'anno precedente. Resta comunque per l'Italia una bassa percentuale di lettori rispetto agli altri Paesi (Spagna, Germania, Stati Uniti, Canada, Francia e Norvegia) come si legge nel Rapporto sullo stato dell'editoria 2017, disponibile al sito: www.illibraio.it (consultato il 04/03/2018). 
relazioni e gli intrecci spazio-temporali. Proprio per questo trattasi di una risorsa, un bene collettivo che avvicina gli uomini nell'esercizio rispettoso dei diritti e dei doveri propri e altrui presupposto per una partecipazione attiva alla vita democratica. Vedere nella pratica della lettura uno strumento di crescita personale, significa anche discutere e riconoscere i valori sociali che rendono coesa e unita una comunità, offrendo inoltre molteplici occasioni ed esperienze di alterità, utili per percepire consapevolmente la presenza dei volti (Lévinas, 2004; Riva, 2008), i messaggi affidati ai linguaggi, le storie narrate da coloro che abitano lo stesso mondo. Questo vuol anche dire superare i confini geografici e le barriere culturali, per un invito alla solidarietà e alla condivisione di quanto è altro da noi stessi e il nostro piccolo e ristretto mondo. Leggere di più, come recita il programma portoghese («Leer+»), serve dunque per essere e saper essere uomini migliori, capaci di critica rispettosa e di autentica saggezza.

\section{Perché leggere}

La lettura è un processo interattivo (Solé, 1993, pp. 25-27), una relazione che il lettore instaura con il testo nel momento in cui lo "interroga", per cercare informazioni e trovare risposte ai problemi quotidiani. Attraverso le pagine scritte, con un dialogo fra lettore e testo, le idee si prestano al confronto e si relazionano, le conoscenze si integrano e si arricchiscono con l'ausilio della memoria e dell'immaginazione. Il nuovo sapere veicolato dal testo consente di incrementare le conoscenze possedute dal lettore, in un gioco infinito fra segni e parole che si rincorrono e si incontrano fra le righe e la mente di chi legge. Questa dialettica rende possibile la personalizzazione del sapere, per "caricarlo" di tutte quelle tonalità emotive che sostengono e sollecitano ogni forma di apprendimento. Esperienze, storie, narrazioni e ricordi si accompagnano sempre alle emozioni, che conferiscono vivacità e sentimento alle decisioni e alle scelte personali. Interesse, sicurezza, motivazione, fiducia nelle proprie competenze e senso di autoefficacia, inoltre, sostengono ed orientano la disponibilità individuale all'apprendimento, anche della lettura (Cfr. Bandura, 1996).

Un insegnante appassionato alla lettura, un genitore e un adulto "buon lettore" rappresentano i migliori esempi per i più piccoli e i giovani, perché la lettura non può essere considerata soltanto un obbligo, anche se questa è la percezione che hanno molti studenti. L'insegnante a volte invita a leggere gli studenti quando sono distratti o disturbano, come se leggere fosse una punizione! Il messaggio che loro recepiscono, chiaramente, è sbagliato. Sono 
pochi, difatti, quegli insegnanti che per premio o ricompensa verso i propri allievi offrono e propongono momenti condivisi di lettura. Il gusto ed il piacere della lettura, invece, merita di essere coltivato, a partire dalla scuola dell'infanzia e primaria, dove leggere deve essere motivo ed occasione di gioco, scoperta, apprendimento e partecipe interesse. All'inizio c'è la fatica, lo sforzo del leggere e riconoscere i suoni delle singole parole e i significati delle frasi, per diventare poi una conquista personale, un successo da vivere con calma e serenità, senza alcuna competizione.

Essere buoni lettori è una competenza che si perfeziona e si acquisisce nel tempo, un traguardo che si consegue con pazienza, coinvolgimento, dedizione, scelte accurate di testi, ma anche con la cura dei luoghi e degli spazi. Questo lo sanno bene le educatrici dei servizi per l'infanzia e le maestre della scuola dell'infanzia che dedicano precisi momenti alla lettura, frutto di una scelta pensata ed attenta, che invita loro a selezionare materiali adatti alle età e agli interessi dei piccoli allievi, nel rispetto dei tempi di apprendimento e di lettura. Non è un caso, difatti, se nelle strutture che accolgono i più piccoli sono presenti spazi distinti per colore e arredamento, "angoli" dove i libri possono essere scelti direttamente dai bambini per la forma, il colore e la materia di cui sono realizzati (in tessuto, con elementi naturali, con accessori musicali, forme componibili, spazi da riempire con espressioni personali). Trasmettere l'interesse e il gusto per la lettura ad incominciare dalla seconda infanzia è sicuramente la buona strada per formare un lettore competente, capace di allenare le abilità intellettuali, nell'atto di comprensione e di interpretazione che la lettura sollecita, sia che venga fatta in solitudine e in silenzio, per «mettere un diagramma temporaneo fra sé e il mondo» (Simone, 1990, p. 31), che in compagnia.

Il buon lettore è colui che, coerentemente ai propri interessi e gusti, sa scegliere i testi ed orientarsi in biblioteca come in libreria. Leggendo e documentandosi riesce a scegliere in base alle differenti tipologie ${ }^{2}$ (narrativa, saggistica, romanzi, poesia, fantasy), perché è la stessa abilità nel leggere che lo indirizza alle letture, nella volontà di soddisfare i propri bisogni e gli interessi culturali. Il buon lettore sa dove cercare nel testo, fra le singole parti, i contenuti che maggiormente lo interessano, per ottenere le informazioni che

\footnotetext{
${ }^{2}$ Sono indicate alcune tipologie, in base alle differenziazioni più comuni. Fra gli studi sul tema si ricordano i seguenti contributi: J.M. Adam (1985), "Réflexion lingüistique sur le types de texte et de compétences en lecture", in L'orientation scolaire et professionale, 14, 4, pp. 293-304; J.A. León y J.A. García Madruga (1989), “Comprensión de textos e instrucción”, in Cuardernos de Pedagogía, 169, pp. 54-59; J.D. Cooper. (1990), Cómo mejorar la comprensión lectora, Visor/Aprendizaje, Madrid; A.M. Kaufman e M.E. Rodriguez (1993), La escuela y los textos, Santillana, Buenos Aires.
} 
vuole, per capire quello che avverte come implicito e non chiaro. Il processo di comprensione è attivo, vitale, affidato alle parole, ai verbi, ai sostantivi, alle espressioni e alla punteggiatura. Attraverso questi segni il soggetto ricostruisce il messaggio per elaborare un proprio senso che integra, arricchisce e rinnova quanto già conosciuto ed esperito, quel background che rappresenta la memoria del «sapere di sfondo rilevante» (Antiseri, 2013, p. 73).

La lettura, dunque, non è un atto meccanico, per essere piuttosto lavoro lento e paziente, con un coinvolgimento di quelle capacità (di analisi, sintesi e di rielaborazione personale) che permettono, appunto, di costruire e delineare nuove risposte di senso, per conferire significati ed esprimere giudizi di valore. Le risposte che il libro può offrire andranno ricercate nella connessione dei fatti, nella sequenza delle storie, nella presentazione di immagini e di parole che permettono di dare forma a nuovi pensieri, per costruire ipotesi sulle quali sostenere nuove forme e modalità di pensiero. Leggere vuol dire anche informarsi, sapere di più circa una disciplina, una realtà da esplorare $\mathrm{e}$ da conoscere che si affida alla penna dello scrittore, con una serie di messaggi sul/al mondo di cui parla anche Ricoeur.

Il lettore competente possiede e coltiva la capacità di "porsi in ascolto del testo", che interpella costantemente, in un'attivazione fra domande e risposte che reciprocamente si delineano, per confrontarsi. Scorrendo le pagine del testo, il lettore, se attento, può capire quello che fin troppo spesso viene dato per ovvio, scontato o implicito, quel "non detto" che, al termine della lettura, assume un significato nuovo, anche inatteso. Il testo «è la parola che permane [...], un archivio a disposizione della memoria individuale e collettiva» (Ricoeur, 2016, p. 135) da comprendere, interpretare e spiegare. Questi atti intenzionali consentono di "entrare" nel testo, per individuarne le idee principali, quelle secondarie e le conclusioni, ma, soprattutto, per adottare un nuovo punto di vista, trovare un' angolazione migliore dalla quale sia anche possibile valutare ed esprimere un proprio giudizio che riguarda in primo luogo il testo e, secondariamente, la capacità individuale di elaborazione dello stesso giudizio. In altre parole, si tratta di riflettere sulle modalità di analisi e comprensione, in uno sforzo meta cognitivo che caratterizza il pensiero maturo. Significativo, inoltre, sarà l'apporto dato dalla lettura nel momento in cui il soggetto si rende conto che ha trascurato o ignorato alcuni aspetti, essenziali per esercitare la propria capacità di giudizio, dalla quale derivano nuove consapevolezze e prospettive. Integrare il contenuto che il testo presenta con quello che il soggetto già sa, rappresenta il percorso della identità narrativa che, ricorda Ricoeur, invita a pensare che il Sé e l'altro che in questo caso sono l'autore o il protagonista del testo e il lettore - sono 
attivamente coinvolti nella progettazione e nella definizione della propria identità (Cfr. Ricoeur, 1986).

La competenza della lettura, dunque, va ben oltre la iniziale codificazione di segni e fonemi, per risultare un atto di scelta, ovvero un processo intenzionale e volontario, lo stesso che guida il soggetto nella preferenza del testo. Sarà proprio quest'ultimo poi a prestarsi ad un commento libero del lettore che nell'opera cerca possibili risposte alle sue domande nel tentativo di trovare soluzione ai problemi (Savater, 2006; Popper, 2001). Leggere, allora, vuol dire stimolare ed esercitare il pensiero (Cfr. Harste e Burke, 1982; Ferreiro e Gómez Palacio, 2013): per rendersi conto di ciò basta pensare alle operazioni compiute nel momento della lettura. Gli stessi processi più semplici e naturali, che sono quelli di sottolineatura di una parte o di un capoverso, la evidenziazione o la marcatura delle parole, la ricerca di definizioni concettuali (parole-chiave) ed anche la stessa scelta di un ordine di lettura dell'intero testo, rappresentano a tutti gli effetti meccanismi del pensiero, strategie messe in atto nelle fasi di analisi, selezione e rielaborazione. Queste sono le comuni modalità attraverso le quali il lettore cerca di capire il contenuto del testo, sperimenta le capacità individuali, acquisisce e mette in atto un metodo di studio e di lettura di cui può personalmente valutare l'efficacia e la rispondenza, sostenuta dall'interesse e dalla motivazione nell'attività svolta. Leggere un quotidiano o un manuale di studio non impegna allo stesso modo, con la medesima intensità, anche se per comprendere il messaggio del testo servono concentrazione ed attenzione. Una lettura veloce e rapida, non sempre denota interesse e volontà di approfondimento, per offrire semmai uno sguardo d'insieme utile per individuare quelle parti che esigono maggiore attenzione, sulle quali successivamente ritornare e soffermarsi. Sicuramente una lettura appassionata ed appassionante regala nuove prospettive, cattura interesse ed attenzione, sollecita curiosità e desiderio, quella forza che permette al lettore di leggere un libro "tutto d'un fiato" per arrivare in breve tempo all'ultima pagina, in modo da contestualizzare e comprendere quello che il testo offre. Una sintesi finale, in tal caso, diventa spunto per un nuovo avvio, nella ricerca di significati che arricchiscono quanto appreso. Una lettura attenta ed impegnata, sostenuta dall'interesse e dalla motivazione, potrà dirsi critica quando potenzia il pensiero strategico, per cui il leggere, lo scrivere, il parlare e l'ascoltare divengono momenti ed occasioni di scambio fra punti di vista e idee (Adler, 2003; Adler e Van Doren, 2013). L'esercizio sinergico di queste competenze permette al lettore di capire, analizzare, descrivere, distinguere e interrogare. Le attività di lettura servono proprio a potenziare la sinergia di saperi, di capacità e di competenze che 
emergono nel momento in cui i soggetti elaborano proprie strategie e producono nuove idee. In questi elementi costruttivi può essere individuato il valore formativo della lettura, che garantisce ai soggetti autonomia e libero pensiero, alla base di scelte personali e comunitarie. Un buon libro stimola il sapere, alimenta il pensiero, genera nuove idee e produce nei soggetti un cambiamento personale che si rivela nel modo di essere e di affrontare la vita, con libertà, creatività e senso di responsabilità (Antiseri, 2013). Se il libro soddisfa le attese del lettore, perché lo sceglie per trovare suggerimenti, proposte alternative in base agli obiettivi individuali, sarà un "buon libro", non tanto perché offre risposte immediate e soluzioni, quanto perché sollecita l'umano "sentire", in accordo con un pensiero volto a ricercare ogni volta connessioni fra idee, teorie e problemi che mobilitano tutte le energie e le motivazioni indispensabili per apprendere. «Alla persona è $[\ldots]$ richiesto di farsi apprendista continuo nei luoghi della quotidianità, di prendersi cura del soggettivo apprendimento, di essere coach di se stessa e di diventarlo sempre più e sempre meglio» (Rossi, 2017, p. 48). In questo «processo di cura dell'esser-già e del poter-essere dell'uomo» (ibidem, p. 50), la lettura fa emergere il contributo che può offrire, nel momento in cui potrà irrobustire la intenzionalità che si esprime nelle scelte, nei propositi e negli impegni, per rivelare così l'autenticità dei soggetti (Cfr. ivi, p. 68).

\section{Buone abitudini: contro la dispersione scolastica}

La lettura, come si è detto, consente alle persone di scegliere e di attribuire un proprio senso alle esperienze fatte: arricchisce e stimola l'introspezione, sollecita la riflessione e il colloquio con se stessi. Inoltre avvicina i soggetti, poiché crea pretesti ed occasioni per confrontarsi con gli altri, con i quali condividere le perplessità, i dubbi, le idee ed i problemi. Sicuramente agevola la formazione di un pensiero maturo, dunque incline al ripensamento e alla ridefinizione di sé. Accrescere il sapere, essere predisposti alla problematizzazione e alla continua riflessività, producono maggiore consapevolezza nei soggetti circa il cambiamento di prospettive e dei punti di vista, aspetti, questi, che sottolineano il valore della lettura, utile anche per offrire nuove aspettative, emozioni ed interessi che mobilitano le capacità riflessive e decisionali delle persone (Cfr. Marchesi Ullastres, 2005, pp. 15-35). Vale allora la pena di chiedersi se la lettura possa anche servire per scoraggiare scelte immediate che spesso sono il frutto di reazioni istintive, di assenza di stimoli, apatia e demotivazione che, stando agli studi, sono da relazionare alla dispersione scolastica. Si tratta di un fenomeno che, malgrado le ultime 
indagini ufficiali che ne dichiarano la attenuazione, potrebbe trovare sostegno nella pratica della lettura. Occorre riflettere sul fatto che se leggendo di più e coltivando maggiori interessi verso le manifestazioni culturali (mostre, musei, cinema, teatro, ecc.) gli studenti possano non cedere con tanta facilità alla tentazione di abbandonare gli studi. Sappiamo che i fattori che influiscono sulla dispersione scolastica sono perlopiù il senso di fallimento, la perdita di interessi e motivazioni, il desiderio di rivendicare una precoce autonomia verso i genitori e, in una considerevole misura, la mancanza di "risposte" da parte delle istituzioni scolastiche ai bisogni degli allievi, i quali avvertono la scuola e i docenti lontani dal loro mondo. I dati forniti recentemente dal Miur prendono in esame una situazione che dovrebbe far riflettere coloro che nella scuola operano. Può essere sufficiente una rivoluzione didattica per coinvolgere gli studenti? E perché è così comune fra i giovani la sensazione di disagio e di rifiuto verso la scuola e le istituzioni? Di che cosa hanno bisogno gli studenti a cui la scuola e i docenti non sanno rispondere? Gli stessi dati avvertono che il bisogno di accettazione, di riconoscimento e di integrazione sociale non viene soddisfatto dalla scuola, realtà che resta distante dal mondo dei più giovani e dalle difficoltà percepite. Se a questi fattori si aggiungono poi le problematiche di natura socio-economica, evidenziate dagli stessi dossier, ecco che il quadro che si delinea è abbastanza negativo, forse più di quanto emerga dagli stessi dati ufficiali. Il problema, che ha una natura culturale oltre che socio-economica, si sposta dalle condizioni concrete ai modelli e alle figure di riferimento, per investire una ricerca che nella sua volontà di fare chiarezza, con la concretezza dei dati, forse trascura alcune questioni.

La lettura, tornando all'argomento in oggetto, se imposta e avvertita come un obbligo non risolve i problemi, non offre soluzioni, ma le buone abitudini possono in un certo senso evitare condizionamenti peggiori. Può essere considerata una buona abitudine quella di leggere? Sicuramente sì, soprattutto quando offre contenuti per riflettere, quando dà spunti per farsi un'idea propria, forte dinnanzi alle spinte all'omologazione, alla perdita di identità e alla arrendevolezza che invita alla pigrizia intellettuale.

Accrescere le possibilità e le modalità di accesso alle forme culturali che la lettura promuove, significa fornire nuovi stimoli, alimentare prospettive e orizzonti aperti che presentano in maniera diversa le esperienze fatte e le scelte intraprese. Soppesare le circostanze, possedere una coscienza più severa e critica della realtà vuol anche dire instaurare un rapporto maggiormente profondo con se stessi, gli altri e le cose. Leggere consente di farsi una 
propria idea, attraverso lo sviluppo e il potenziamento degli strumenti cognitivi, affettivi e sociali, sui quali si allena la competenza meta cognitiva, importante per orientare le azioni individuali.

La lettura di un libro, da considerare dunque una sana e buona abitudine, permette al lettore, indipendentemente dalla sua età, di nutrire aspettative, di modificare punti di vista, per provare anche nuove emozioni e sentimenti che concorrono a rafforzare le abilità logiche e argomentative, attraverso i processi di rielaborazione, di catalogazione e di generalizzazione delle informazioni (Cfr. Petrini, 1990, p. 194). In ragione di ciò la lettura deve essere diritto e possibilità per tutti, in quanto promuove abilità intellettuali, sociali e culturali utili per lo sviluppo e la crescita personale. Leggere è modalità di apertura all' esperienza dell'alterità, con uno sforzo di comprensione dei molteplici linguaggi, delle storie, delle esperienze, dei tempi e degli spazi degli altri. Viaggiare nei mondi e nelle realtà differenti dalle proprie, condividere emozioni e sentimenti è quanto la lettura può offrire, in un impegno, sostenuto dallo sforzo intellettuale, che guida nella decifrazione dei segni e dei simboli che animano e 'vivono' nelle pagine, articolati nei capoversi, strutturati nei paragrafi e nei capitoli, per una condivisione di messaggi che rende efficace la comunicazione fra lettore e autore.

Le osservazioni di cui si è dato conto, invitano dunque a ricordare il ruolo della lettura nei processi di crescita umana, connotati dal saper prendere scelte in maniera autonoma per elaborare proprie visioni del mondo e della vita. Ecco allora che la lettura può rappresentare strumento di consolidamento ed esercizio di pensiero, per avviare una rivoluzione culturale (Cfr. Marchesi Ullastres, 2005) a favore di una società democratica, costituita da uomini liberi che sanno pensare con la propria testa, perché inclini alla riflessione, desiderosi di essere informati per capire, valutare e scegliere (Antiseri, 2013; Berti, 2011; Severino, 2012). In ragione di ciò possiamo affermare che la pratica della lettura, che abbiamo definito una "buona abitudine", da acquisire e sollecitare fra i giovani, può rappresentare uno strumento di cui dispone ogni società per cambiare, evolversi in senso civile e democratico con il riconoscimento ed il rispetto di altri modi di pensare, di vivere e di credere, tratti nei quali affiora la diversità e, dunque la singolarità, di ogni persona.

\section{Conclusioni}

L'impegno che anima le politiche istituzionali di molti paesi, attivamente coinvolti nelle campagne di promozione della lettura, trova legittimazione 
nel suo riconoscimento come risorsa, prezioso strumento di evoluzione culturale e di progresso socio-economico. Le istituzioni nazionali, gli organismi deputati all'interesse e al benessere delle comunità, insieme alle associazioni ufficiali e non, credono ed investono nel potere della lettura, sulla quale scommettono per assicurare a tutti la possibilità di farsi una propria idea, con la quale esprimere l'immaginazione e un pensiero maturo indispensabile per dilatare lo spazio e il tempo che come uomini condividiamo, con tutte le difficoltà che emergono e le possibili incomprensioni.

\section{Riferimenti bibliografici}

Adam J.M. (1985), "Réflexion lingüistique sur le types de texte et de compétences en lecture", L'orientation scolaire et professionale, 14, 4: 293-304.

Adler M.J. (2003), Saper parlare, saper ascoltare, Armando, Roma.

Adler M.J. e Von Doren C. (2013), Come leggere un libro. Per essere un lettore competente, Sovera Edizioni, Roma.

Antiseri D. (2013), Dalla parte degli insegnanti, La Scuola, Brescia.

Bandura A. (1996), Il senso di autoefficacia. Aspettative su di sé e azione, Erickson, Trento. Berti E. (2011), Invito alla filosofia, La Scuola, Brescia.

Casey Carter S. (2016), Quando la scuola educa. 12 progetti formativi di successo, trad .it., Città Nuova Editrice, Roma (tit. orig. On Purpose. How Great School Cultures Form Strong Character, Corwin Press (2011), United States).

Cooper J.D. (1990), Cómo mejorar la comprensión lectora, Visor/Aprendizaje, Madrid.

Ferreiro E. y Gómez Palacio M., eds. (2013), Nuevas perspectivas sobre los procesos de lectura y escritura, Siglo XXI, México.

Forum del libro, a cura di (2015), "Esperienze internazionali di promozione della lettura", Quaderni di Libri e Riviste d'Italia, Roma.

Goleman D. e Senge P. (2016), A scuola di futuro. Manifesto per una nuova educazione, trad. it., Rizzoli Etas, Milano.

Harste J. and Burke C. (1982), Predictibilidad en lecto-escritura, pp. 64-66, in E. Ferreiro y M. Gómez Palacio, eds., Nuevas perspectivas sobre los procesos de lectura y escritura, Siglo XXI, 2013, México.

León J.A. y García Madruga J.A. (1989), “Comprensión de textos e instrucción”, Cuardernos de Pedagogía, 169: 54-59.

Lévinas E. (2004), Totalità e infinito. Saggio sull'esteriorità, trad.it., Jaca Book, Milano.

Marchesi Ullastres A. (2005), "La lectura como estrategia para el cambio educativo", Revista de Educación, núm. extraordinario: 15-35.

Morin E. (2015), Insegnare a vivere. Manifesto per cambiare l'educazione, trad.it., Cortina, Milano.

Morin E. (2001), I sette saperi necessari all'educazione del futuro, trad. it., Cortina, Milano.

Morin E. (2016), 7 lezioni sul pensiero globale, trad. it., Cortina, Milano.

Morin E. (1999), La testa ben fatta. Riforma dell'insegnamento e riforma del pensiero, trad. it., Cortina, Milano.

Petrini E. (1990), La nascita del lettore, in R. Simone, a cura di, Un mondo da leggere, La Nuova Italia, Firenze. 
Popper K.R. (1996), Tutta la vita è risolvere problemi, Rusconi, Milano; rst. Bompiani, 2001, Milano.

Kaufman A.M. and Rodriguez M.E. (1993), La escuela y los textos, Buenos Aires, Santillana. Ricoeur P. (2016), Che cos'è un testo, in Dal testo all'azione. Saggi di ermeneutica, trad. it., Milano, Jaca Book (ed. orig., Qu'est-ce qu'un texte? Expliquer et comprendre, in AA.WW., Hermeneutik und Dialektik, J.B.C. Mohr, 1970, Tübingen).

Ricoeur P. (1986), Tempo e racconto I, trad. it., Jaca Book, Milano (ed. orig. Temps et récit I, Seuil, 1983, Paris).

Riva F., a cura di (2008), E. Lévinas, G. Marcel, P. Ricoeur. Il pensiero dell'altro, Edizioni Lavoro, Roma.

Rossi B. (2017), Educare all'ammirazione. Di sé, dell'altro, della Terra, Pensa Multimedia, Lecce.

Severino E. (2012), Educare al pensiero, La Scuola, Brescia.

Simone R., a cura di (1990), Un mondo da leggere, La Nuova Italia, Firenze.

Savater F. (2006), Le domande della vita, trad.it., Laterza, Roma-Bari.

Solé I. (1993), "Estrategias de comprension de lectura y aprendizaje", Cuadernos de Pedagogía, 216: 25-27.

Siti web consultati:

www.illibraio.it

www.planonacionaldeleitura.gov.pt

www.rizzolieducation.it

www.miur.gov.it/web/guest/pubblicazioni

www.miur.gov.it/dispersionescolastica

www.ilsole24ore.com

www.tuttoscuola.com

www.hubmiur.pubblicaistruzione.it

www.indire.it

www.istat.it/archivio/lettura

www.sololibri.net

www.tpi.it

www.unesdoc.unesco.org 


\title{
Le pratiche di lettura nelle scuole dell'infanzia e primarie umbre
}

\author{
di Marco Bartolucci e Adriana Timpone
}

\section{Perché la lettura fa bene}

Numerosi sono gli aspetti positivi associati alla lettura che si possono trovare in letteratura scientifica. Esaminando gli aspetti strettamente cognitivi, processare un elemento di narrazione va ben al di là della semplice comprensione linguistica. Leggere e comprendere una storia significa innescare numerosi e diversi meccanismi nella cognizione umana (Mar, 2004). I lettori rappresentano ciò che il testo descrive attraverso immagini mentali. Queste rappresentazioni sono anche note come "modelli situazionali" e incorporano informazioni sullo spazio, sul tempo, sui rapporti causali, sui personaggi (Zwaan e Radvansky, 1998). Pertanto, le regioni cerebrali coinvolte nella comprensione del testo sono necessariamente molteplici e non solo strettamente linguistiche: probabilmente, qualsiasi rete che supporta il linguaggio, la memoria, e persino la percezione gioca un ruolo in questo processo (Mar, 2006).

In generale, l'atto di leggere attiva, a livello cerebrale, un vasto network di aree (Batini e Bartolucci, 2015), operando a diversi livelli cognitivi, e costituendo una vera e propria "palestra" per diverse funzioni, come ad esempio le funzioni mnestiche ma anche quelle emotive (Billington et al., 2012), le funzioni esecutive e la velocità di elaborazione dell'informazione (Uchida e Kawashima, 2008).

Per sua natura l'essere umano è fruitore e creatore di storie. Dapprima le ascoltiamo, poi impariamo a leggerle e a narrarle. Attraverso le storie conosciamo il mondo che ci circonda, entriamo in contatto con la cultura di appartenenza, costruiamo la nostra identità, diamo un senso agli eventi ponendoli in un certo ordine. Se pensiamo all'attività della lettura, il primo scopo che ci viene in mente è sicuramente l'acquisizione di informazioni. È altret- 
tanto vero, però, che la lettura non è finalizzata solo all'apprendimento diretto, ovvero non si legge solo per imparare. Per molte persone, infatti, la lettura rappresenta un'attività che si svolge per il semplice piacere di farlo, un valido modo per impiegare il proprio tempo libero.

Quando una persona si avvicina ad un testo scritto, gli effetti che quest'ultimo avrà sulla persona sono innumerevoli e, a volte, impercettibili a prima vista. Ciò che viene trasmesso al lettore è molto più della semplice informazione veicolata dal testo, anche perché il modo in cui il testo è recepito «dipende tanto dal lettore quanto dal testo stesso. La lettura, infatti, non è da intendere come una diretta e automatica interiorizzazione [...] perché non è affatto un processo a senso unico: essa è interazione dinamica tra testo e lettore» (D'Alessandro e Domanin, 2005, p. 19).

Mentre leggiamo e diamo un senso a ciò che è scritto, la lettura stessa modella noi, la nostra relazione con gli altri e con il mondo. Apre la nostra mente e ci fa addentrare verso ampi e sconosciuti orizzonti. Ci fa viaggiare con la fantasia e andare al di là del reale. Ci fa conoscere e identificare con tanti personaggi diversi. La lettura è un "antidoto" alla manipolazione narrativa altrui (della pubblicità, della politica), è una difesa dal "pensiero unico" (che costituisce il "seme" del pregiudizio), è uno strumento di costruzione e ricostruzione del Sé e della propria vita, incrementa le competenze utili alla gestione del proprio futuro, potenzia le capacità di comprensione di se stessi e degli altri, rinforza le capacità di immaginazione (Batini, Toti e Bartolucci, 2016).

I benefici della lettura sono quindi innumerevoli: dall'empowerment della literacy (Clark e Douglas, 2011), dell'intelligenza verbale e delle conoscenze generali sul mondo (Cunningham e Stanovich, 1998), allo sviluppo sociale della persona (Taylor, 1970).

Quando il lettore si immerge in una storia, in qualche modo si immedesima in ognuno dei suoi personaggi, cerca di capire i suoi scopi e di provare i suoi sentimenti. La capacità di attribuire stati mentali a se stessi e agli altri viene definita "Teoria della Mente" o "Mentalizing" ed è «una competenza fondamentale che permette le complesse relazioni sociali che caratterizzano le società umane» (Kidd e Castano, 2013). Una vera e propria "simulazione semplificata della realtà» (Mar e Oatley, 2008) dove le complesse dinamiche sociali che si svolgono nel nostro mondo vengono riprodotte nel testo in modo realistico, ma semplificato, che ci aiuta a capirle e ad entrare nella mente dei protagonisti. 


\subsection{La lettura come strumento di sviluppo in tenera età}

È oramai noto che la lettura per/con i bambini è una pratica fondamentale per lo sviluppo del bambino e per la condivisione di emozioni con i genitori (Cambi, 2012). Numerosi sono gli studi sui benefici delle pratiche di lettura durante i primi anni di vita, sia dal punto di vista dello sviluppo cognitivo (Horowitz-Kraus, Vannest e Holland, 2013; Speer et al., 2009; Hutton et al., 2015; Sénéchal e LeFevre, 2002) che di creazione di legami e condivisione emotiva tra chi legge e chi ascolta (Aram e Aviram, 2009; Catarsi, 2011; Cambi, 2012; Ronfani et al., 2006).

La lettura è un'attività complessa che coinvolge diverse aree della persona: l'area socio-affettiva, l'area cognitiva e l'area comunicativa, essa sollecita lo sviluppo delle capacità critiche e analitiche, il gusto estetico e l'espressione emozionale (Golfetto, 2007). Permettere al bambino di ascoltare liberamente storie, fiabe o eventi reali, senza la fatica di dover comprendere un testo scritto, è molto importante durante i primi anni di scolarizzazione, non solo per migliorare le capacità di comprensione del testo. Nell'ascolto della lettura, i bambini vivono, traendone piacere, un'esperienza linguisticamente e cognitivamente complessa che predispone ad abilità cognitive altrettanto complesse di manipolazione dei simboli verbali (Cardarello, 1995). Tale pratica favorisce lo sviluppo della comprensione di un testo e l'acquisizione di un vocabolario ricco, l'implementazione di significati, la restituzione di senso e la comprensione di micro-avvenimenti legati alla vita quotidiana, oltre ad una serie di esperienze emotive (Batini e Giusti, 2010). Attraverso sessioni giornaliere di lettura si sviluppano capacità come il pensiero narrativo, l'ascolto e la concentrazione, lasciando ampio spazio all'immaginazione e alla creatività.

Altro elemento fondamentale è la funzione sociale della lettura, che attraverso lo sviluppo dell'empatia e della condivisione delle emozioni con i compagni, aumenta le capacità di espressione e narrazione.

Ruolo fondamentale è ricoperto dalla motivazione e dal coinvolgimento dei bambini nel processo di lettura. Le ricerche individuano quattro requisiti fondamentali che associano la motivazione alla lettura: il libero accesso ad un'ampia scelta di libri presenti in classe; l'opportunità per i bambini di scegliere da soli i libri che vogliono leggere; la familiarità con i libri che non appaiono come oggetti estranei ma perfettamente integrati nelle attività quotidiane; la possibilità di interagire socialmente con altri, siano essi pari o adulti, durante la lettura (Gambrell, 1996). 
Nell'ascolto della lettura i bambini fanno piacevolmente, traendone diletto, un'esperienza linguisticamente e cognitivamente complessa che predispone ad abilità cognitive complesse di manipolazione dei simboli verbali (Cardarello, 1995). L'ascolto di testi scritti, infatti, potenzia, a più livelli, le capacità linguistico-cognitive dei bambini, che interverranno anche nell'apprendimento delle capacità strumentali, e che rappresentano una competenza importante per la comprensione e l'apprezzamento della lettura.

Il potenziamento del linguaggio, a cui ci si riferisce genericamente quando si sostiene che l'ascolto aiuti a sviluppare lo stesso, non riguarda dunque il potenziamento delle sole capacità lessicali, ma anche di quelle sintattiche, immaginative, inferenziali che, nel loro insieme, costituiscono fondamentali supporti per affrontare la lettura vera e propria.

Diversi studi hanno dimostrato come i lettori vadano incontro ad una crescita empatica durante la lettura, considerando la possibilità che il testo narrato e i suoi personaggi consentano di fare esperienza indiretta di interazioni sociali, delle quali vengono mostrare anche le conseguenze.

Numerosi studi attestano il ruolo della simulazione motoria anche nella lettura della finzione narrativa, affermando che l'assunzione di una prospettiva empatica è una parte usuale del coinvolgimento del lettore con la finzione narrativa (Coplan, 2004). Lo sviluppo dell'empatia è un ingrediente importante nello sviluppo socio-emozionale. La conoscenza emozionale fornisce infatti ai bambini informazioni riguardo le situazioni e le norme comportamentali in uso nella società (Denham et al., 2003).

\section{Abitudini di lettura in Italia e a scuola}

L'ultima analisi ISTAT pubblicata, stima che in Italia vi sia un calo progressivo dei lettori: si passa dal 42\% del 2015 (popolazione dai 6 anni in su) al 40,5\% nel 2016 (percentuale di chi ha risposto di aver letto almeno un libro negli ultimi 12 mesi, per motivi non strettamente connessi al lavoro o allo studio). Tra questi si distinguono i lettori "deboli" e "forti" rispettivamente il $45,5 \%$ e il $13,7 \%$. Se si analizza poi il dato per fasce di età, l'unica che supera il 50\% è quella tra gli 11 e i 19 anni. Il sud segna l'area geografica più critica con solo il $28,8 \%$ di lettori.

Il decremento dei lettori è principalmente legato a una diminuzione di quelli deboli, che con più facilità si trasformano in "non lettori". L'ostacolo alla lettura sembra essere la mancanza di tempo e l'accesso limitato ai libri per bambini. La mancanza di tempo porta alcuni adulti a distaccarsi progressivamente dalla lettura, mentre il limitato accesso, che può essere o meno 
una diretta conseguenza della mancanza di tempo, va di pari passo con la scarsità di luoghi in cui viene promossa la lettura di testi per l'infanzia.

Chiaramente il mancato avvicinamento alla lettura in tenera età può essere dannosa, in quanto studi mostrano come le abitudini di lettura apprese in questa età hanno una diretta corrispondenza sulle abitudini di lettura da adulti: la maggior parte dei "lettori forti" ha avuto genitori che, nei primi cinque anni di vita, hanno dedicato tempo alla lettura ad alta voce (Duursma et al., 2008). Oltre a questo si affiancano altre cause, come lo status socio economico della famiglia: i bambini che crescono in famiglie a basso reddito hanno meno accesso a libri cartacei e a momenti di lettura condivisa e questo ha ovvie ripercussioni sull'alfabetizzazione e sulle future potenzialità di lettura dei bambini (McCormick, 1977; Wolf, 2009).

La padronanza delle abilità di lettura è da sempre considerata uno dei principali obiettivi della scuola, il saper leggere infatti è ritenuto essenziale per i successivi apprendimenti, dato che gran parte del processo di apprendimento-insegnamento avviene tramite la lingua scritta e in maniera particolare tramite la lettura di testi.

Il tema dell'insegnamento della lettura è legato ad una problematica di ampie proporzioni che ha trovato anche in Italia molto spazio sia nel dibattito culturale, sia nel campo della ricerca teorica e pedagogico-didattica. Tuttavia risulta complesso capire se, a questa consapevolezza diffusa nella società dell'importanza della lettura nei processi formativi dell'individuo - sia come strumento per affrontare felicemente il curricolo degli studi, sia come strumento indispensabile per non essere discriminato in una società sempre più complessa e selettiva nei confronti degli individui scarsamente alfabetizzati - corrisponda una consapevolezza altrettanto adeguata nelle scuole, soprattutto in relazione alle strategie e alle pratiche didattiche.

Oggi la scuola rappresenta il luogo fondamentale per l'incontro con la lettura e ricopre un ruolo importante per formare "autentici" lettori, desiderosi di ripercorrere autonomamente questa esperienza. Il problema centrale diventa quello di educare al gusto della lettura, di sviluppare una forte motivazione personale a instaurare un libero incontro con il libro, durante il quale ogni ragazzo si lasci coinvolgere e trasportare dentro la realtà narrata.

Un buon lettore si costruisce nel tempo, attraverso un lungo percorso che inizia dall'infanzia, età in cui il bambino incontra il libro e lo esplora con l'aiuto di una voce esterna che legge. La scuola dell'infanzia può essere considerata una sorta di ponte fra i genitori e la scuola primaria, in cui è necessario che gli insegnanti siano mediatori fra i bambini e il testo scritto, in quanto a sei anni - quando incominceranno la scuola primaria - avranno già acquisito una grande varietà di informazioni. In un secondo momento, 
quando il soggetto ha appreso a leggere, il ruolo dell'insegnante rimane comunque di rilievo, poiché è attraverso di esso che il bambino può alimentare la sua motivazione a leggere, confrontandosi con l'insegnante su contenuti, personaggi, luoghi e significati delle storie, poiché egli giocherà un ruolo fondamentale nell'aiutare i bambini a divenire lettori che leggeranno sia per piacere che per reperire informazioni (Gambrell, 1996).

\section{I risultati della ricerca in Umbria}

L'obiettivo dell'indagine qui presentata è analizzare le abitudini di lettura dei docenti delle scuole dell'infanzia e primarie dell'Umbria. La ricerca è stata condotta dalla cattedra di Pedagogia sperimentale del dipartimento FISSUF dell'Università degli studi di Perugia, nell'anno accademico 2017/2018. I questionari sono stati somministrati nel mese di novembre 2017, grazie alla collaborazione delle studentesse e degli studenti frequentanti il corso ${ }^{1}$, a gran parte delle scuole dell'infanzia e primarie del territorio umbro ${ }^{2}$.

\footnotetext{
${ }^{1}$ Hanno partecipato alla ricerca i seguenti studenti del corso di Pedagogia Sperimentale dell'anno accademico 2017/18: Lucia Accorroni, Alessandra Alfredini, Agnese Arcangeli, Elena Baglioni, Ilaria Baglioni, Roberta Balloni, Rebecca Balucani, Giulia Basso, Aurora Bastioli, Elena Batella, Giulia Beccafichi, Federica Bellucci, Lucia Biagetti, Gregorio Bianchini, Nicole Bianchini, Lucia Binario, Beatrice Bitocchi, Marika Bosi, Agnese Bozzalla, Giulia Brizi, Rosa Brizzi, Diana Bruno, Marta Bugattelli, Benedetta Cardia, Vanessa Cavalletti, Lucia Cavargini, Aurora Ceccarelli, Giulia Ceccarelli, Francesca Cerati, Chiara Cerquiglini, Eleonora Cesarini, Sofia Cinfrignini, Gabriele Corradi, Angela De Nicola, Giulia De Vergori, Giulia Deibianchi, Erima Del Principe, Daniele Di Lisa, Ylenia Di Vito, Eleonora Dominici, Martina Dominici, Annalisa Finocchi, Mirella Fiorucci, Martina Furno, Valeria Gentili, Elena Giunti, Andrea Giustiniani, Glenda Gnagni, Francesca Gorini, Gloria Grandolini, Giulia Iorio, Laura Lana, Ilaria Lanterna, Giorgia Lauri, Sofia Mafucci, Beniamino Manoni, Clara Mapelli, Matteo Marcelli, Margherita Marchini, Beatrice Mariano, Maria Marzocchella, Sofia Marzoli, Prisca Mastini, Angelica Meloni, Miriam Mencarelli, Linda Mengoni, Sara Minciaroni, Vanessa Monacelli, Giovanna Montalto, Benedetta Monteleone, Linda Morelli, Catia Morosi, Michela Moscatelli, Francesca Niglio, Marta Padoin, Maddalena Palermi, Federica Passerini, Martina Pasteni, Aurora Pauselli, Gemma Pellegrini, Ylenia Pettinelli, Erika Poderini, Gloria Presicce, Valentina Profidia, Chiara Radi, Camilla Rampini, Tommaso Reali, Marina Rellini, Eleonora Renzetti, Sofia Riganelli, Laura Ripa, Michela Rosati, Rebecca Rossi, Giada Rossi, Marina Ruffini, Arianna Santarelli, Pasquale Santonastaso, Ilaria Saraca Volpini, Elena Saracini, Marta Schianin, Alice Servettini, Valentina Sgombra, Federica Sgrigna, Sara Sportellini, Sara Spurio Passamonti, Gloria Tinti, Claudia Tomassoni, Federica Trampolini, Ilenia Traversini, Elena Uccellani, Virginia Umena, Benedetta Venturi, Camilla Vivenzio, Giulia Volpi, Veronica Zangarelli.

${ }^{2}$ Hanno preso parte all'indagine i seguenti istituti: IC Perugia 1; IC Perugia 3; IC Perugia 4; IC Perugia 5; IC Perugia 6; IC Perugia 8; IC Perugia 11; IC Perugia 12; IC Perugia 14; $1^{\circ}$
} 
Il questionario, implementato ad hoc, presenta la seguente struttura, finalizzata ad indagare alcune dimensioni fondamentali connesse al fenomeno in esame:

1) informazioni generali;

2) esperienza professionale;

3) attitudine personale alla lettura;

4) lettura in famiglia;

5) lettura in classe;

6) organizzazione;

7) contenuti;

8) percezione;

9) obiettivi della lettura;

10) considerazioni finali.

Per quanto riguarda la prima parte del questionario, ovvero la parte anagrafica, possiamo notare che, il campione, composto da 598 rispondenti, presenta una netta prevalenza di insegnanti donne $(96,5 \%, \mathrm{n}=577)$. L'età media è di 47 anni. Un terzo dei docenti insegna con il diploma magistrale, mentre il $42,1 \%$ ha una laurea magistrale o di vecchio ordinamento (V.O.). Circa il $41 \%$ degli insegnanti ha due figli; il $44 \%$ ha un'anzianità di servizio al di sotto dei 16 anni.

Circolo Didattico di Città di Castello; $2^{\circ}$ Circolo Didattico di Città di Castello; IC Leonardo da Vinci (San Giustino); $1^{\circ}$ Circolo Didattico di Marsciano; $2^{\circ}$ Circolo Didattico di Marsciano; IC di Sigillo; $1^{\circ}$ Circolo Didattico di Gubbio; $2^{\circ}$ Circolo Didattico di Gubbio; $3^{\circ} \mathrm{Cir}-$ colo Didattico di Gubbio; IC G. Marconi (Terni); IC Alto Orvietano (Fabro); Direzione Didattica di Todi; IC di Gualdo Tadino; IC Bevagna-Cannara (Foligno); $2^{\circ}$ Circolo Didattico di Foligno; Direzione Didattica F. Rasetti (Castiglion del Lago); IC Torgiano-Bettona (Torgiano); IC di Orvieto-Montecchio (Orvieto); Direzione Didattica di Bastia Umbra; IC Narni Scalo (Narni). 


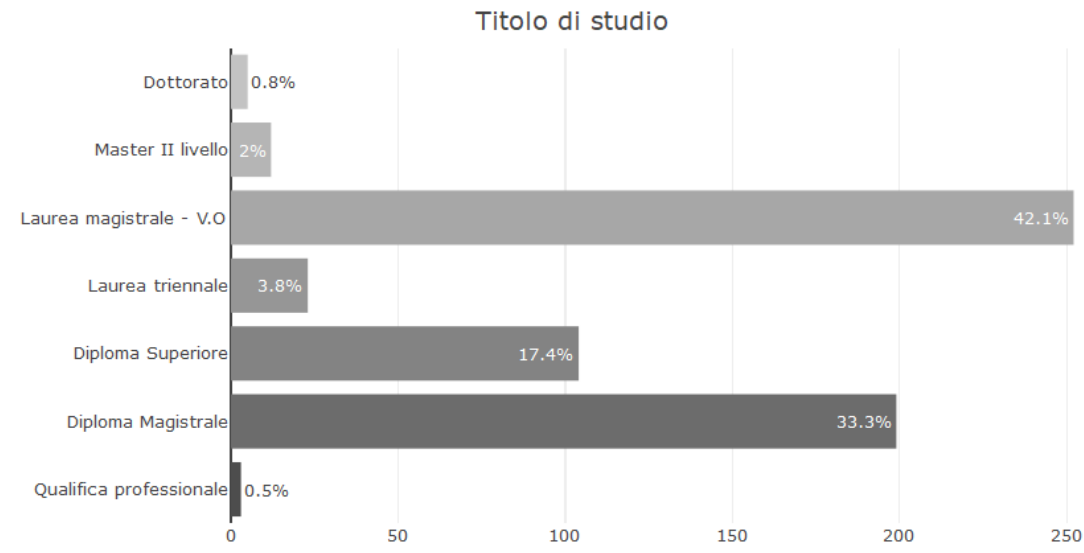

Fig. 1 - Risultati relativi alla domanda "Titolo di studio"

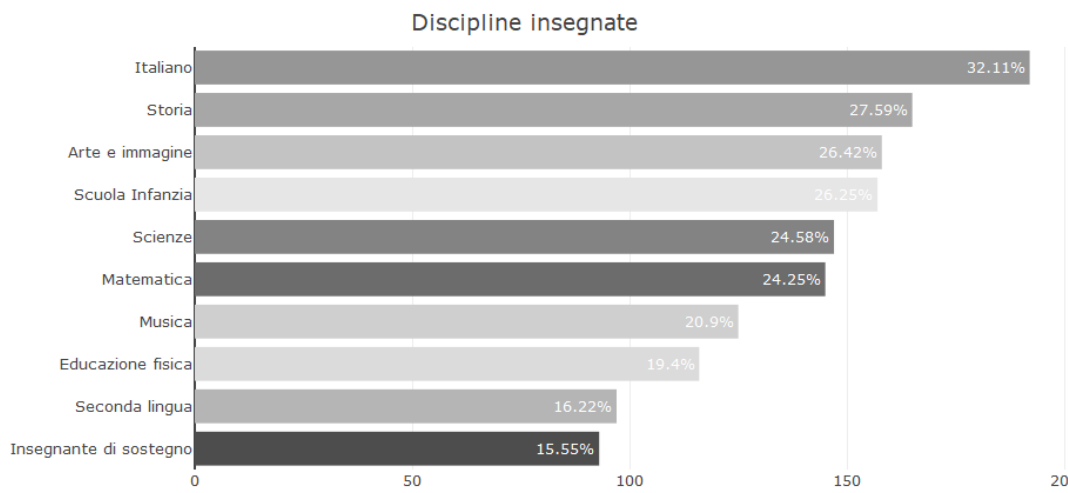

Fig. 2 - Risultati relativi alla domanda "Discipline insegnate"

Il 24\% dei rispondenti insegna in una scuola dell'infanzia $(n=143)$. Per la sola scuola primaria, un terzo dei rispondenti insegna Italiano (il 32,11\% sul totale delle materie insegnate). Si noti che ciascun docente può insegnare più materie. Circa il $16 \%$ del campione è rappresentato da insegnanti di sostegno. 


\section{Partecipazione a corsi di aggiornamento}

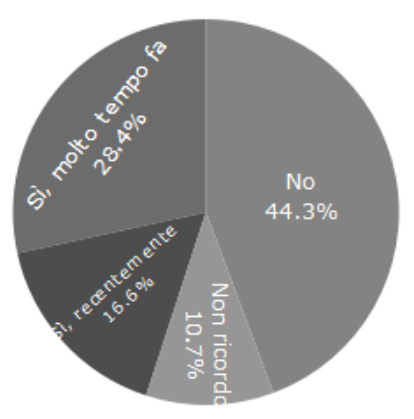

Fig. 3 - Risultati relativi alla domanda "Partecipazione a corsi di aggiornamento sulla lettura"

Quasi la metà dei docenti $(44,3 \%)$ non ha mai partecipato a corsi di aggiornamento sulla lettura (dato che non cambia se filtriamo i dati per scuola dell'infanzia e primaria).

Il 66,5\% dei docenti afferma di amare molto la lettura. La media dei libri letti in un anno è di circa 8 libri per piacere e 5 per formazione professionale.

Oltre 1' $80 \%$ dei rispondenti predilige il supporto cartaceo.

La seconda parte del questionario si concentrava sulle abitudini di lettura in famiglia, nello specifico si chiedeva ai rispondenti come avessero avvicinato i figli alla lettura. I dati mostrano che lo strumento più utilizzato è la semplice presenza dei libri in casa, seguita dalla lettura condivisa serale. 


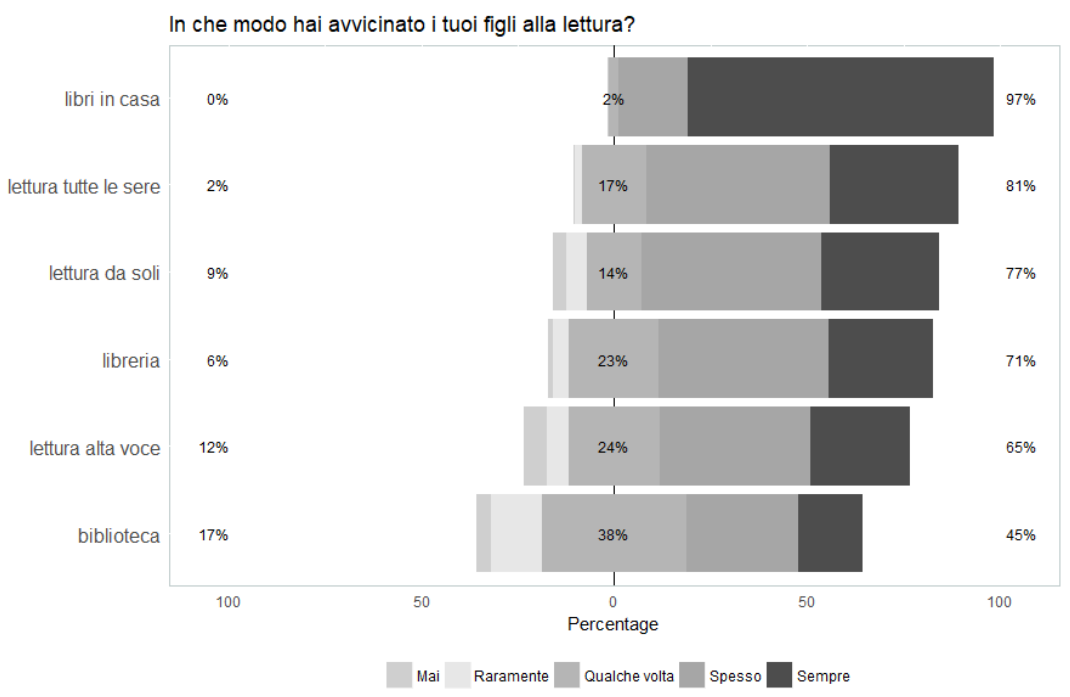

Fig. 4 - Risultati relativi alla domanda "In che modo hai avvicinato $i$ tuoi figli alla lettura"

Un'ulteriore sezione indagava l'organizzazione della lettura in classe. La quasi totalità dei docenti $(96 \%)$ dichiara di leggere ad alta voce in classe. Di questi, un quarto $(24,7 \%)$ lo fa più volte al giorno e non c'è un particolare momento della giornata prediletto per la lettura in classe.

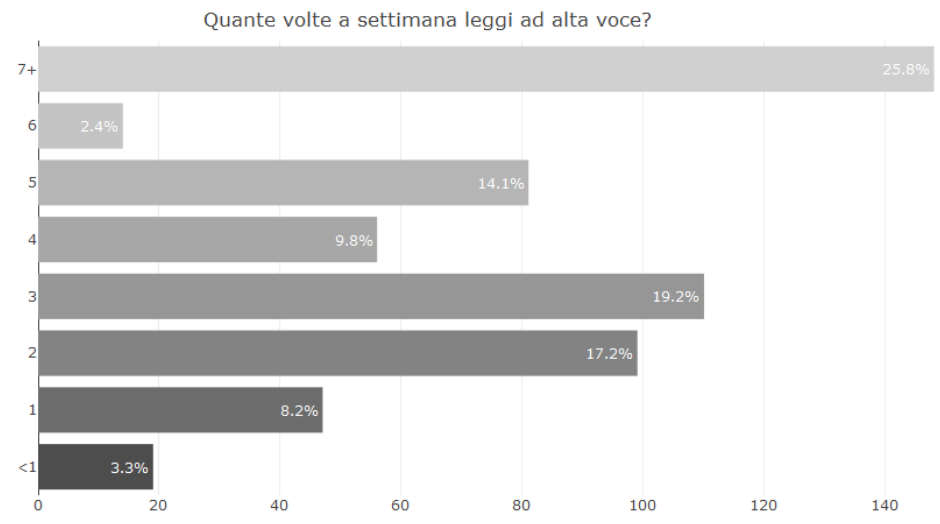

Fig. 5 - Risultati relativi alla domanda "Quante volte a settimana leggi ad alta voce" 


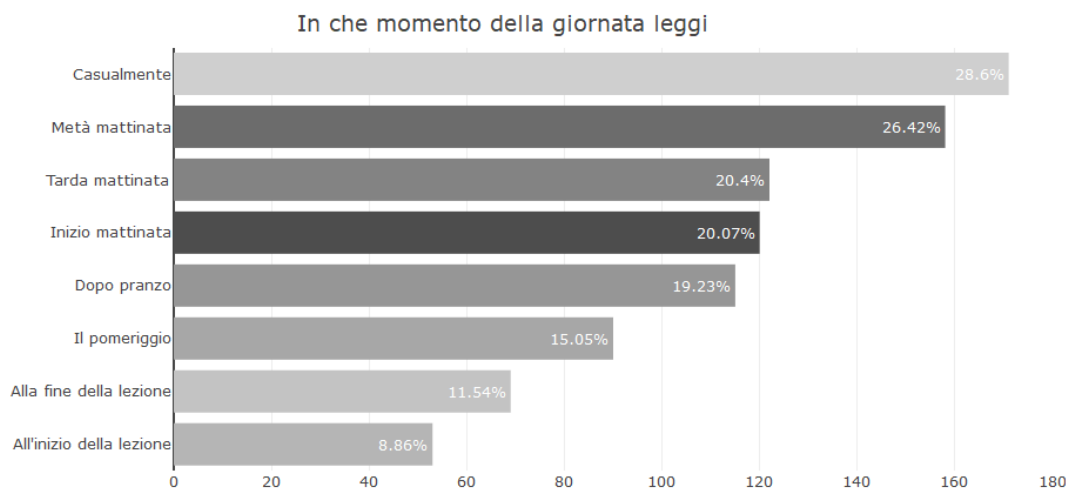

Fig. 6 - Risultati relativi alla domanda "In che momento della giornata leggi"

La stragrande maggioranza dei docenti dichiara, inoltre, di leggere per non più di trenta minuti consecutivi (meno di 20 minuti il 74\% degli intervistati), dato che non cambia sostanzialmente tra scuola primaria e scuola dell'infanzia.

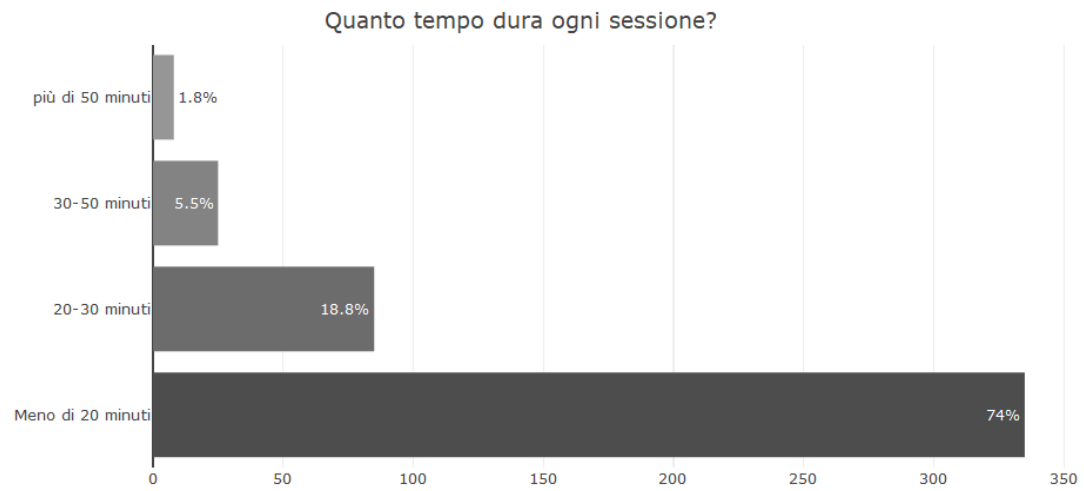

Fig. 7 - Risultati relativi alla domanda "Quanto tempo dura ogni sessione" relativa alla domanda precedente "In che momento della giornata leggi"

La frequenza settimanale sembra, nella maggioranza del campione, attestarsi intorno alle $2 / 3$ volte, anche se un quarto dei rispondenti dichiara di leggere anche più di 7 volte. 


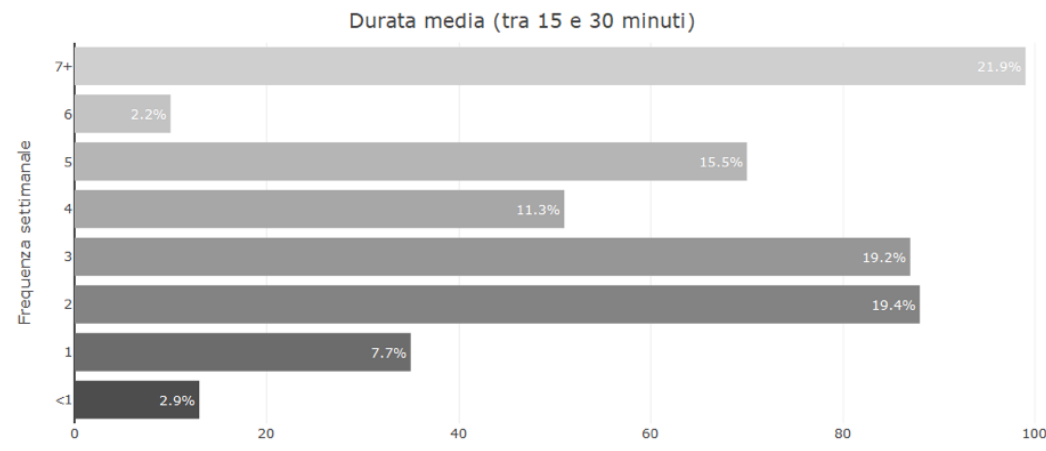

Fig. 8 - Risultati relativi alla domanda "Con che frequenza settimanale leggi" filtrati per i rispondenti che alla domanda "quanto dura ogni sessione" hanno risposto fra 15 e 30 minuti

Le storie lette richiedono più incontri solo "qualche volta" per più della metà dei rispondenti ed il setting di lettura prediletto sembra essere "seduti ai banchi" oppure "seduti in cerchio" con una netta variazione fra gli ordini scolastici: infanzia "seduti in cerchio" $89,2 \%$; primaria "seduti ai banchi" $62,3 \%$.

\section{Leggi storie che richiedono più incontri?}

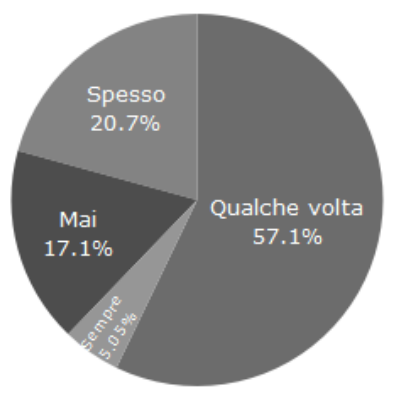

Fig. 9 - Risultati relativi alla domanda "Leggi storie che richiedono più incontri" 


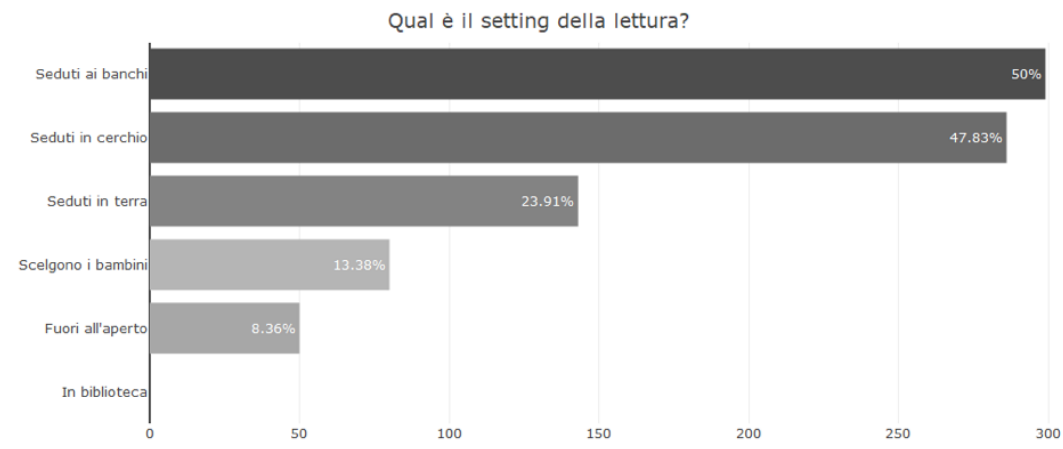

Fig. 10 - Risultati relativi alla domanda "Quale è il setting della lettura"

Vengono spesso utilizzati stimoli visivi durante la lettura, con una maggioranza di utilizzo di immagini cartacee e disegni.

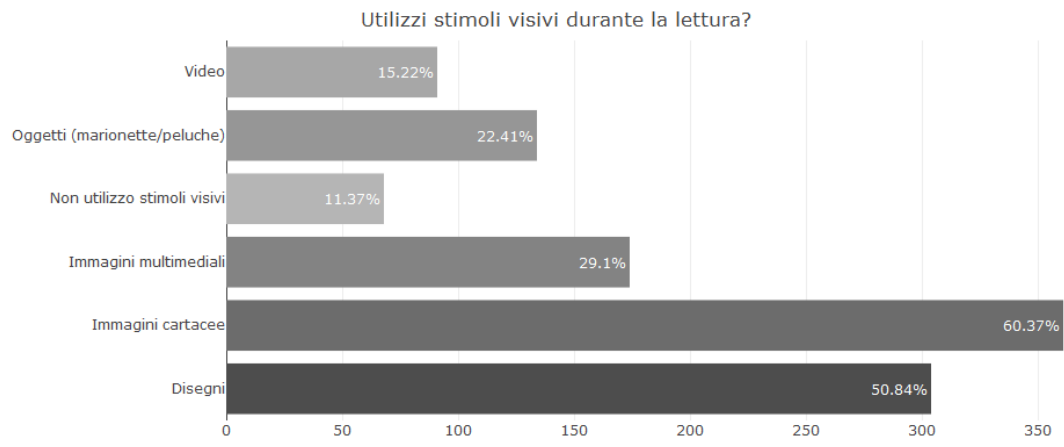

Fig. 11 - Risultati relativi alla domanda "Utilizzi stimoli visivi" 


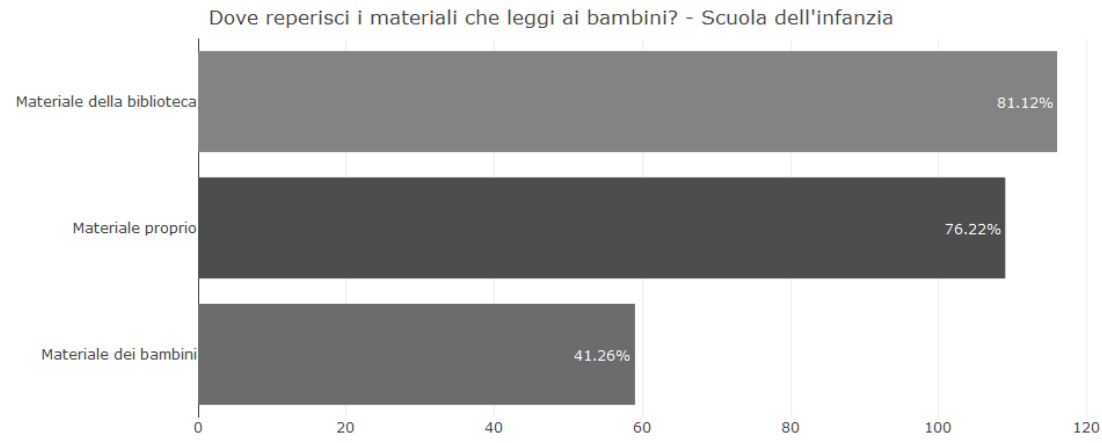

Fig. 12 - Risultati relativi alla domanda "Dove reperisci i materiali da leggere" filtrati per rispondenti insegnanti della scuola dell'infanzia

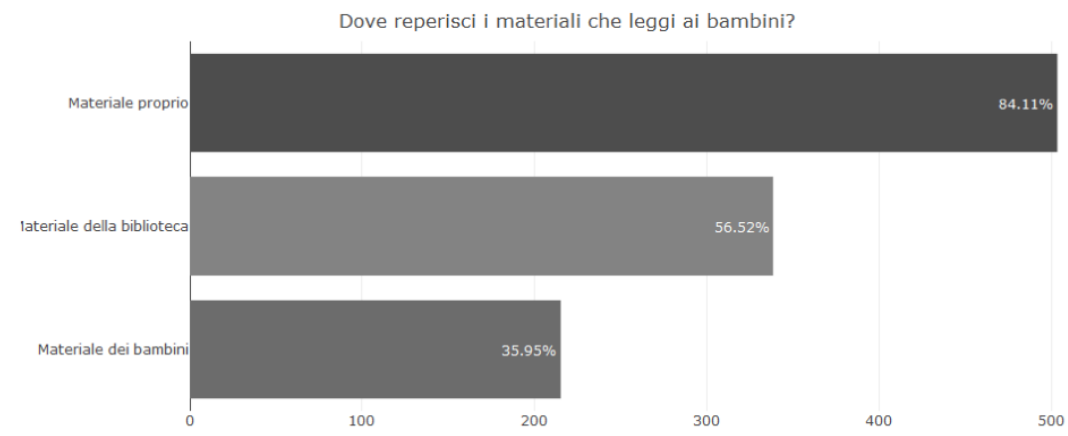

Fig. 13 - Risultati relativi alla domanda "Dove reperisci i materiali da leggere" filtrati per rispondenti insegnanti della scuola primaria

Riguardo al reperimento di materiali, i materiali portati e suggeriti dai bambini vengono utilizzati in entrambi gli ordini di scuola, anche se in maniera marginale rispetto al materiale della biblioteca, che nella scuola dell'infanzia si attesta all' $82 \%$ circa, e al materiale proprio che nella scuola primaria si attesta all' $86,6 \%$. 


\section{Sono previste attività legate alla lettura anche con la presenza di esperti esterni?}

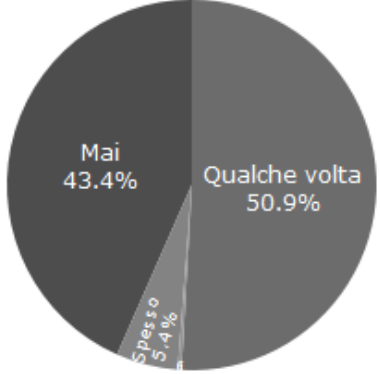

Fig. 14 - Risultati relativi alla domanda "Sono previste attività legate alla lettura anche con la presenza di esperti esterni"

In entrambi gli ordini le attività di lettura sono previste anche con la presenza di esperti esterni solo nel $50 \%$ dei casi, e spesso (49,3\%) o sempre $(22 \%)$ sono associate alla didattica, denotando, soprattutto per quel che riguarda la scuola primaria, la scarsità di momenti in cui si propongono letture a livello di semplice piacere narrativo.

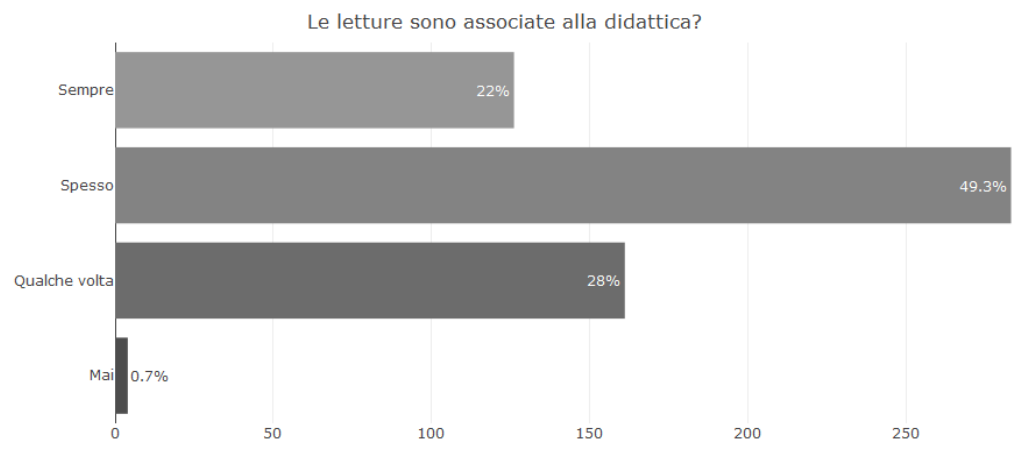

Fig. 15 - Risultati relativi alla domanda "Le letture sono associate alla didattica" 


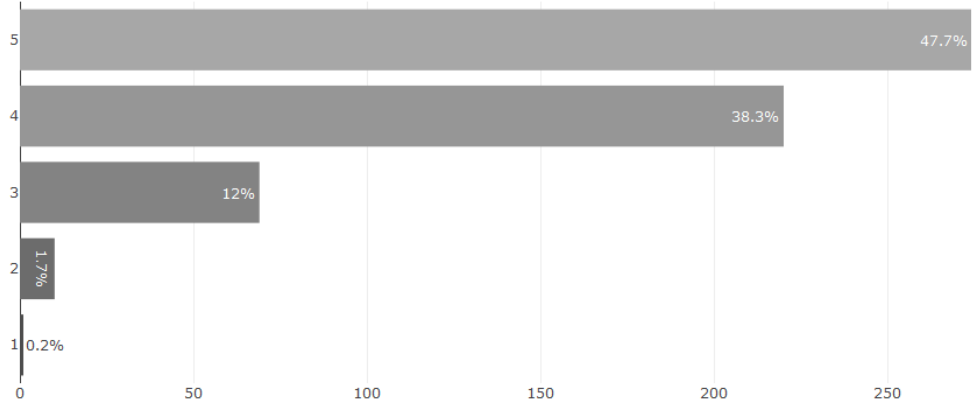

Fig. 16 - Risultati relativi alla domanda "É un'attività che piace ai tuoi alunni"

Sebbene strettamente legata alla didattica, la lettura sembra comunque essere una pratica molto gradita dagli alunni di entrambi gli ordini di scuola.

Nell'ultima parte del questionario, si è invece andati ad indagare quelli che sono gli obiettivi che si prefiggono gli insegnanti nell'utilizzo della lettura con i ragazzi. Al primo posto si pensa che possa stimolare sopratutto la fantasia e la creatività, con al secondo posto le competenze di ascolto e quelle linguistiche. Al quarto il potenziamento cognitivo, metre agli ultimi posti della "classifica" troviamo il potenziamento della memoria, l'acquisizione del punto di vista di altri e lo svago.

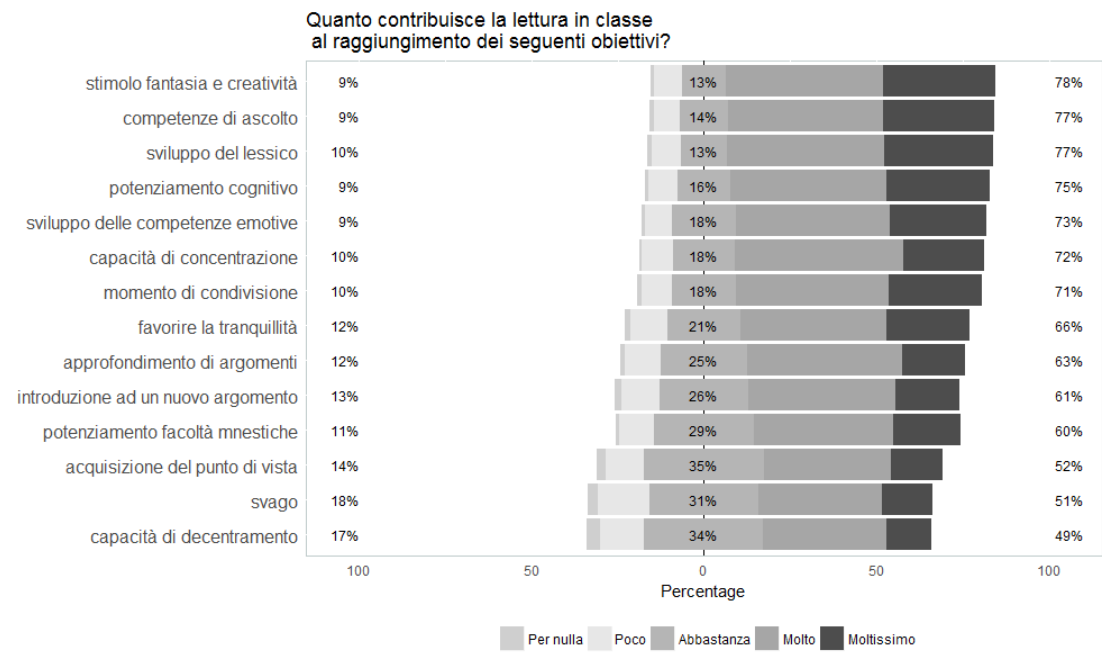

Fig. 17 - Risultati relativi alla domanda "Quanto contribuisce la lettura in classe al raggiungimento dei seguenti obiettivi" 


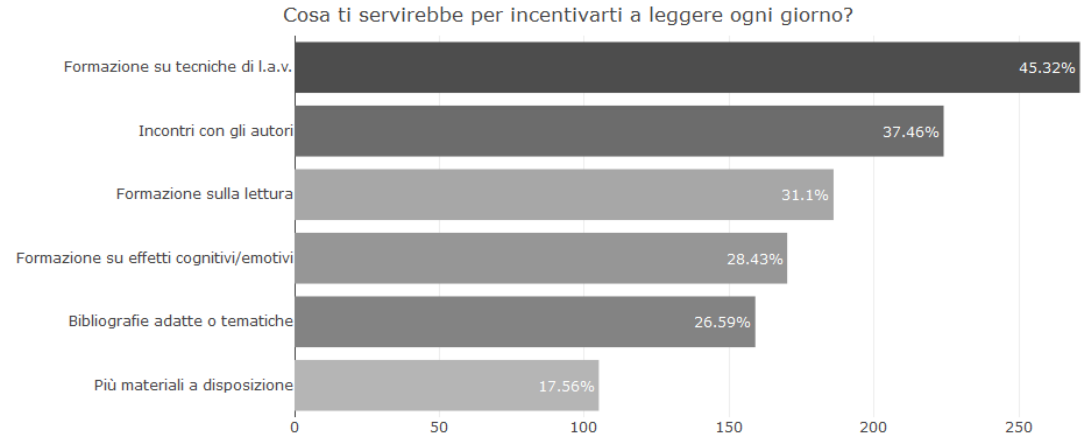

Fig. 18 - Risultati relativi alla domanda "Cosa ti servirebbe per incentivarti a leggere ogni giorno"

Gran parte dei docenti $(45,32 \%)$, infine, ritiene che una formazione specifica sulle tecniche della lettura ad alta voce possa costituire un incentivo all'esercizio costante di lettura in classe.

\section{Conclusioni}

I dati qui riportati mostrano un panorama abbastanza incoraggiante su quelle che sono le abitudini degli insegnanti e gli atteggiamenti riguardo la lettura in generale e la sua applicazione nel contesto scolastico.

Dalle risposte dei partecipanti si evincono dati in linea con la letteratura scientifica, ad esempio risulta come sia forte l'influenza del contesto familiare e l'accessibilità ai libri come indicatore di quanto i bambini possano avvicinarsi alla lettura. Sebbene la quasi totalità dei docenti dichiari di leggere ad alta voce in classe, la frequenza di tale attività risulta comunque marginale e presente in media solo 2 o 3 volte a settimana. La mancanza di un particolare momento nella giornata dedicato alla lettura, indica, inoltre, quanto questa pratica non sia strutturata e quindi ancora inserita casualmente all'interno del curricolo scolastico. Allo stesso modo, la durata dei momenti di lettura è limitata e molto probabilmente poco associata a scopi didattici specifici, perdendo quindi di rilevanza. Questo dato è supportato anche dal fatto che solo qualche volta le letture (della stessa storia/romanzo) vengono protratte per più incontri consecutivi. Inoltre, specie nella scuola primaria, anche il setting preferito (quello seduti ai banchi) fa pensare che la lettura sia un mezzo didattico per gli insegnanti, piuttosto che una specifica attività, che 
richiederebbe, per la condivisione dei contenuti, la fruibilità e la partecipazione, setting invece piu vicini a quelli utilizzati nella scuola dell'infanzia, come l'essere seduti in cerchio.

Che la lettura non venga ritenuta e utilizzata come una specifica attività di empowerment di varie competenze e dimensioni personali, lo si evince anche dal fatto che solo marginalmente i ragazzi sono stimolati alla ricerca personale e alla condivisione dei propri gusti e delle proprie scelte letterarie, così come la discussione di gruppo, come metodo di verifica della comprensione del testo utilizzato, si situa soltanto al terzo posto.

I rispondenti rendono esplicito il fatto che la lettura sia solo un'attività "accessoria" (il 70\% del campione ritiene che la lettura è spesso o sempre associata alla didattica).

Nonostante queste modalità, la lettura è molto gradita dagli studenti, che forse ne "avvertono" i benefici, indicando un indice di gradimento molto alto.

I dati relativi agli obiettivi che la lettura si prefigge, mostrano in generale che gli/le insegnanti ritengono che tale pratica sia benefica per molte dimensioni, sopratutto quelle cognitive, del lessico, delle competenze di ascolto e della creatività. Questi dati sono supportati, come menzionato nei precedenti paragrafi, da molti studi in letteratura scientifica, quindi si potrebbe ipotizzare che un'adeguata formazione per gli insegnanti possa poi favorire l'instaurarsi di questa attività a pieno titolo nel curricolo scolastico, piuttosto che configurarsi come un'attività accessoria. In un'ottica verticale, questo porterebbe, come dimostrato in letteratura, allo sviluppo e potenziamento di varie abilità a seconda del livello di età, in una modalità continua, su molte dimensioni personali: dalle competenze di ascolto alla concentrazione e allo sviluppo emotivo nell'infanzia, al ragionamento critico, competenze lessicali, funzioni esecutive, empatia e resilienza nei gradi successivi, accompagnando quindi l'alunno in differenti momenti di vita e di sviluppo. In quest'ottica, inoltre, ogni alunno sarebbe stimolato sin dall'infanzia a sviluppare i propri gusti personali riguardo la lettura, favorendo l'installarsi di tale attività come routine forte all'interno della vita scolastica ed extrascolastica dell'alunno.

\section{Riferimenti bibliografici}

Aram D. and Aviram S. (2009), “Mothers' storybook reading and kindergartners' socioemotional and literacy development", Reading Psychology, 30, 2: 175-194.

Batini F. e Giusti S. (2010), Imparare dalle narrazioni, Unicopol, Milano.

Batini F. e Bartolucci M. (2015), "Paper or Facebook? An experiment on the comprehension of texts with a group of dropouts",Form@re, 15, 1: 40. 
Batini F., Toti G. and Bartolucci M. (2016), "Neuropsychological benefits of a narrative cognitive training program for people living with dementia: A pilot study", Dementia \& Neuropsychologia, 10, 2: 127-133.

Billington J., Carroll J., Davis P., Healey C. and Kinderman P. (2012), “A literature-Based Intervention for Older People Living with Dementia", Perspect Public Health, 133, 3:165-73.

Catarsi E., a cura di (2011), Educazione alla lettura e continuità educativa, Edizioni Junior, Bergamo.

Cambi F. (2012), "Genitori e figli attorno al libro", Rivista Italiana di Educazione Familiare, 7, 2: 23-27.

Cardarello R. (1995), Libri e bambini. La prima formazione del lettore, La Nuova Italia, Firenze.

Clark C. and Douglas J. (2011), "Young People's Reading and Writing: An In-Depth Study Focusing on Enjoyment, Behaviour, Attitudes and Attainment", National Literacy Trust.

Coplan A. (2004), "Empathic engagement with narrative fictions", The Journal of aesthetics and art criticism, 62, 2: 141-152.

Cunningham A.E. and Stanovich K.E. (1998), "What reading does for the mind", American educator, 22: 8-17.

D'Alessandro P. and Domanin I. (2005), Filosofia dell'ipertesto: esperienza di pensiero, scrittura elettronica, sperimentazione didattica. Apogeo Editore.

Denham S.A., Blair K.A., DeMulder E., Levitas J., Sawyer K., Auerbach-Major S. and Queenan P. (2003), "Preschool emotional competence: Pathway to social competence?", Child development, 74, 1: 238-256.

Duursma E., Augustyn M. and Zuckerman B. (2008), "Reading aloud to children: the evidence", Archives of disease in childhood, 93, 7: 554-557.

Gambrell L.B. (1996), "Creating classroom cultures that foster reading motivation", The reading teacher, 50, 1: 14.

Golfetto G. (2007), "Educare alla lettura: dal lettore emozionale alla familiarizzazione con la lettura", Lombello Soffiato D., a cura di, «Novel» e «romance»: strumenti per l'analisi dei generi letterari in prospettiva pedagogica, Cleup, 35-52.

Horowitz-Kraus T., Vannest J.J. and Holland S.K. (2013), "Overlapping neural circuitry for narrative comprehension and proficient reading in children and adolescents", Neuropsychologia, 51, 13: 2651-2662.

Hutton J.S., Horowitz-Kraus T., Mendelsohn A.L., DeWitt T., Holland S.K. and C-MIND Authorship Consortium (2015), "Home reading environment and brain activation in preschool children listening to stories", Pediatrics, 136, 3: 466-478.

Kidd D.C., and Castano E. (2013), "Reading literary fiction improves theory of mind", Science, 342, 6156: 377-380.

Mar R.A. (2004), "The neuropsychology of narrative: story comprehension, story production and their interrelation", Neuropsychologia, 42, 10: 1414-1434.

Mar R.A., Oatley K., Hirsh J., dela Paz J. and Peterson J.B. (2006), "Bookworms versus nerds: Exposure to fiction versus non-fiction, divergent associations with social ability, and the simulation of fictional social worlds", Journal of Research in Personality, 40, 5: 694-712.

Mar R.A. and Oatley K. (2008), "The function of fiction is the abstraction and simulation of social experience", Perspectives on psychological science, 3, 3: 173-192.

McCormick S. (1977), "Should you read aloud to your children?", Language Arts, 54, 2: 139163.

Ronfani L., Sila A., Malgaroli G., Causa P., Manetti S., Garofolo T. and AIB A.I.B. (2006), La promozione della lettura ad alta voce in Italia. Quaderni acp, 1. 
Sénéchal M. and LeFevre J.A. (2002), "Parental involvement in the development of children's reading skill: A five-year longitudinal study", Child development, 73, 2: 445-460.

Speer N.K., Reynolds J.R., Swallow K.M. and Zacks J. M. (2009), "Reading stories activates neural representations of visual and motor experiences", Psychological science, 20, 8: 989-999.

Taylor D.M. (1970), "Explanation and meaning”.

Uchida S. and Kawashima R. (2008), "Reading and solving arithmetic problems improves cognitive functions of normal aged people: a randomized controlled study", Age, 30, 1: 21-29.

Wolf M. (2009), Proust e il calamaro. Storia e scienza del cervello che legge, Vita e Pensiero, Milano.

Zwaan R.A. and Radvansky G.A. (1998), "Situation models in language comprehension and memory", Psychological bulletin, 123, 2: 16. 


\section{Lettura e intercultura nelle scuole dell'infanzia e primarie dell'Umbria}

di Roberta Balloni, Ylenia Di Vito e Laura Lana

\section{Introduzione}

\subsection{Popolazione scolastica di origine straniera in Italia}

La scuola italiana è oggi una realtà multietnica e multiculturale. Multietnica per la presenza di una pluralità di etnie diverse, multiculturale per la pluralità di modelli culturali di riferimento che si manifestano in stili di vita, orientamenti e comportamenti disomogenei (Besozzi, 2006).

Secondo gli ultimi dati forniti dal Miur (riferiti all'anno scolastico 2015/2016), sono quasi 815.000 gli alunni con cittadinanza non italiana presenti nelle classi, dalla scuola dell'infanzia alla secondaria di II grado, il $9,2 \%$ del totale della popolazione scolastica. Di questi, quasi il $60 \%$ sono nati nel nostro Paese. Negli ultimi cinque anni, dal 2011/2012 al 2015/2016, l'incremento delle seconde generazioni è stato del $43,2 \%$. È tra i banchi della primaria che si ha il maggior numero di presenze.

\subsection{La risposta della scuola italiana: l'educazione intercul- turale}

La scuola italiana ha risposto alla presenza di studenti con cittadinanza straniera con la proposta di un'educazione interculturale che tagli trasversalmente tutte le discipline della scuola e si proponga di modificare percezioni e convinzioni “sull'Altro". L'educazione interculturale va, quindi, intesa non come una nuova disciplina a sé stante, ma come un'ottica diversa con cui guardare tutti i saperi disciplinari (Besozzi, 2006). 
Nel 2007 l'Osservatorio nazionale per l'integrazione degli alunni stranieri e per l'educazione interculturale, promosso dal Miur, ha pubblicato $L a$ via italiana per la scuola interculturale e l'integrazione degli alunni stranieri rivolgendosi a dirigenti scolastici ed insegnanti. Nel documento si evidenziano due dimensioni dell'educazione interculturale su cui gli educatori devono soffermarsi. La prima mira ad ampliare il campo cognitivo, fornendo informazioni e promuovendo la capacità di decentramento, con l'obiettivo di mostrare la varietà di punti di vista da cui osservare una situazione. La seconda va ad agire sul piano affettivo e relazionale attraverso il contatto, la condivisione di esperienze, il lavoro per scopi comuni, la cooperazione, in quanto «il pregiudizio più radicato non viene messo in dubbio soltanto dalla smentita alle proprie opinioni» (Miur, 2007, p. 17).

\subsection{La lettura come strumento di educazione intercultu- rale}

Piano cognitivo, affettivo e relazionale trovano nell'esperienza della lettura perfetta sintesi. Nel 2014 il Miur ha emanato le Linee guida per l'accoglienza e l'integrazione degli alunni stranieri. Tra le indicazioni fornite nel documento per promuovere la dimensione multilingue e multiculturale delle classi, particolare risalto è dato alla lingua. Tra le modalità di riconoscimento e valorizzazione della diversità linguistica, viene annoverato l'uso di storie bilingui, perché «attraverso la diffusione di fiabe del mondo in versione bilingue o plurilingue, si possono far conoscere a tutti gli alunni esempi di un patrimonio narrativo ampio ed intrecciato e presentare alla classe lingue, scritture e alfabeti differenti» (Miur, 2014, p. 19).

In molte scuole è ormai presente da anni lo scaffale multiculturale di promozione della lettura, un'esperienza ideata da Vinicio Ongini negli anni ' 90 e avvalorata dal Miur in tutti i più recenti documenti normativi. Lo scaffale accoglie libri di divulgazione, fiabe e storie, testi nelle lingue d'origine (di narrativa e divulgazione), bilingui e plurilingui, autobiografie degli immigrati e degli emigrati italiani, strumenti per l'avviamento ai testi e i dizionari nelle diverse lingue, video e cd rom multimediali sulle diverse lingue e culture prodotti dall'editoria (Luatti, 2008). 


\subsection{II potere interculturale della lettura ad alta voce}

È soprattutto la lettura ad alta voce che si configura come strumento potente di accoglienza ed inclusione capace di operare un apprendimento significativo veicolato dalle emozioni. Frezza (2016) utilizza la metafora della "marea": grazie alla lettura ad alta voce l'ascoltatore riesce ad essere contemporaneamente qui e altrove, nell'aula e dentro i luoghi della narrazione, va e viene e, nel momento del ritorno, porta con sé sentimenti, emozioni, vissuti per condividerli con gli altri e stabilire il confronto con esperienze sconosciute o culturalmente opposte. L'esperienza della lettura ad alta voce permette, infatti, dopo la narrazione vera e propria, di condividere domande e far venir fuori spunti da cui aprire temi e problemi e cercare altri libri che possano rispondervi. Il tutto in un ambiente protetto, nel quale l'alunno può affrontare le proprie paure giocando ad una "simulazione" della realtà per poi tornare ad essa più forte di nuove risposte.

È proprio in questo scambio che si può costruire il concetto di rispetto, «inteso come considerazione di quella persona-portatrice di valori ed esperienze da apprezzare e tutelare come patrimonio comune. Perché se il patrimonio diventa comune, comune sarà la sua valorizzazione e comune lo sviluppo del senso di appartenenza inteso come partecipazione attiva nei confronti di una comunità» (Frezza, 2016, p. 99).

\subsection{I libri interculturali}

La letteratura interculturale conta oggi decine e decine di pubblicazioni, dagli albi illustrati per la scuola dell'Infanzia alle memorie di migranti per i più grandi.

Bertazzo (2017) fornisce alcune interessanti indicazioni su come scegliere un libro interculturale. Innanzitutto, ciò che non deve avere: una categorizzazione della diversità semplicistica (stereotipi, tratti attribuiti a gruppi specifici, etc.); una diversificazione concettuale per coppie di opposti (vero/falso, normale/diverso, nero/bianco, ricco/povero, etc.); una visione etnocentrica, che presenta le altre culture proiettando criteri, valori e modelli della propria. Fondamentale, invece, che le opere scelte sappiano dare una rappresentazione della diversità che permetta un decentramento del proprio punto di vista, che allarghi i confini culturali decostruendo stereotipi, stimolando domande, scambi e conflitti. 
Ogni storia è sempre il risultato dell'intreccio con altre storie, altri racconti: non esistono storie uniche, monoculturali. Perché il libro ci restituisce la normalità e la ricchezza di noi stessi e del mondo di cui siamo parte. Così come nella vita, anche nella narrativa le storie si contagiano e, con loro, le grammatiche del linguaggio e delle culture.

Ma, soprattutto, un libro deve essere bello.

Una volta, quando mi è stato chiesto come deve essere un libro interculturale, ho risposto "Deve essere bello!". Non basta che ci siano alcuni ingredienti, come esser stato scritto da un autore straniero, essere tradotto e provenire da altri paesi, essere scritto in lingue diverse dall'italiano o bilingue. Conta anche che sia una bella storia, che sappia incuriosire, contano le illustrazioni e la traduzione e, soprattutto, conta come viene letto o raccontato, in quale contesto, da chi e per quale bambino (Ongini 2007, p. 32).

\section{Presentazione della ricerca}

\subsection{Popolazione scolastica di origine straniera in Umbria}

In Umbria risiedono 95.935 abitanti di origine straniera (il 10,8\% della popolazione complessiva); la percentuale di stranieri è più alta rispetto alla media italiana $(8,3 \%)$, ma in linea con quella del Centro Italia $(10,7 \%)$. Gli alunni stranieri sono 16.945 su una popolazione scolastica regionale complessiva di 123.118 unità, pari al 13,8\% del totale. L'Umbria è la terza regione italiana con più alta incidenza di studenti stranieri, dopo Emilia Romagna e Lombardia (IDOS, 2017; Caritas-Migrantes, 2017).

\subsection{II questionario}

I risultati qui presentati derivano da un'indagine conoscitiva condotta dalla cattedra di Pedagogia sperimentale del dipartimento FISSUF dell'Università degli studi di Perugia, nell'anno accademico 2017/2018. I questionari sono stati somministrati nel mese di novembre 2017 - grazie alla collaborazione delle studentesse e degli studenti frequentanti il corso - a gran parte degli istituti scolastici dell'infanzia e primaria del territorio umbro. Obiettivo dell'indagine: indagare le abitudini di lettura dei docenti. 
La rilevazione è stata condotta attraverso l'uso di un questionario messo a punto dal gruppo di ricerca con la partecipazione delle studentesse e degli studenti. Lo strumento di rilevazione presenta la seguente struttura, finalizzata ad indagare alcune dimensioni fondamentali connesse al fenomeno in esame: 1) informazioni generali; 2) esperienza professionale; 3) attitudine personale alla lettura; 4) lettura in famiglia; 5) lettura in classe; 6) organizzazione; 7) contenuti; 8) percezione; 9) obiettivi della lettura; 10) considerazioni finali.

In questa sede ci focalizzeremo su alcuni quesiti in particolare, relativi alla scelta di libri provenienti da altre culture.

\subsubsection{II campione}

Il campione è composto da 598 rispondenti, presenta una netta prevalenza di insegnanti donne $(96,5 \%, \mathrm{n}=577)$. L'età media è di 47 anni. Un terzo dei docenti insegna con il diploma magistrale; il $42,1 \%$ ha una laurea magistrale o Vecchio ordinamento. Circa il $41 \%$ degli insegnanti ha due figli. Per quanto riguarda l'esperienza professionale, circa il $44 \%$ dei docenti ha un'anzianità di servizio al di sotto dei 16 anni. Il 24\% dei rispondenti insegna in una scuola dell'infanzia $(\mathrm{n}=143)$. Per la sola scuola primaria, un terzo dei rispondenti insegna Italiano (il $32,11 \%$ sul totale delle materie insegnate). Si noti che ciascun docente può insegnare più materie. Circa il 16\% del campione è rappresentato da insegnanti di sostegno.

\section{Risultati}

Prima di tutto, è stato importante capire il contesto e lo spazio narrativo nel quale si muovono i docenti intervistati. Agli insegnanti è stato, infatti, chiesto cosa leggessero di solito ai loro alunni (fig. 1). Dalle loro risposte è emerso che i racconti sono il genere prediletto per la lettura in classe $(67 \%)$, seguiti da fiabe/favole (56\%), storie con figure $(55,5 \%)$, filastrocche $(43 \%)$, poesie $(32,9 \%)$, libri consigliati dai bambini $(29,6 \%)$; in coda i grandi classici $(9,8 \%)$, i romanzi $(4,5 \%)$ e i testi teatrali $(4 \%)$. 


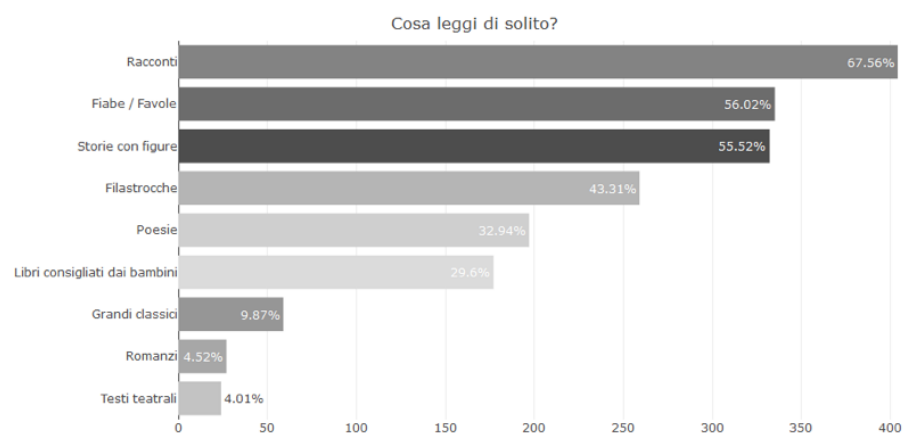

Fig. 1 - Consuetudini di lettura in classe

Entrando nello specifico dei due ordini di scuola, si evincono alcune differenze: i docenti dell'Infanzia prediligono fiabe/favole (88\%) e storie con figure (83\%), data anche l'età degli alunni (fig. 2).

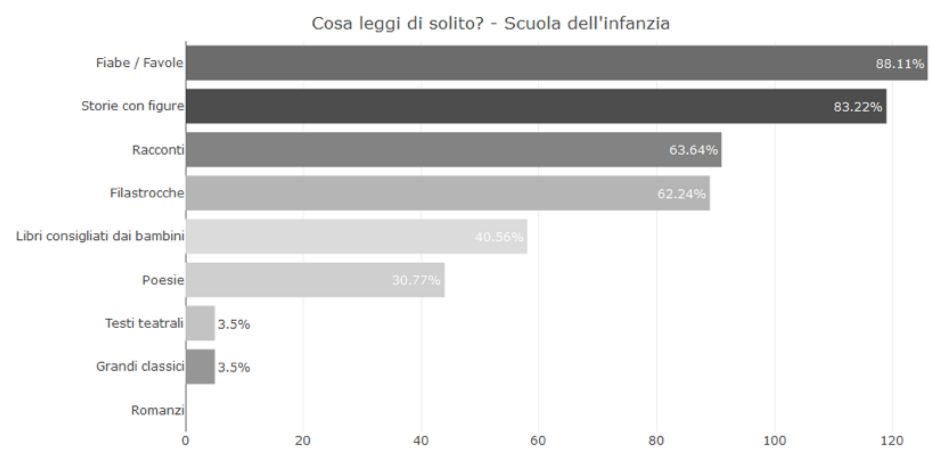

Fig. 2 - Consuetudini di lettura in classe nella scuola dell'infanzia

I docenti della primaria optano, invece, fortemente per i racconti (68\%). In entrambi i casi, si posizionano di nuovo in coda i testi teatrali, i grandi classici e i romanzi (fig. 3). 


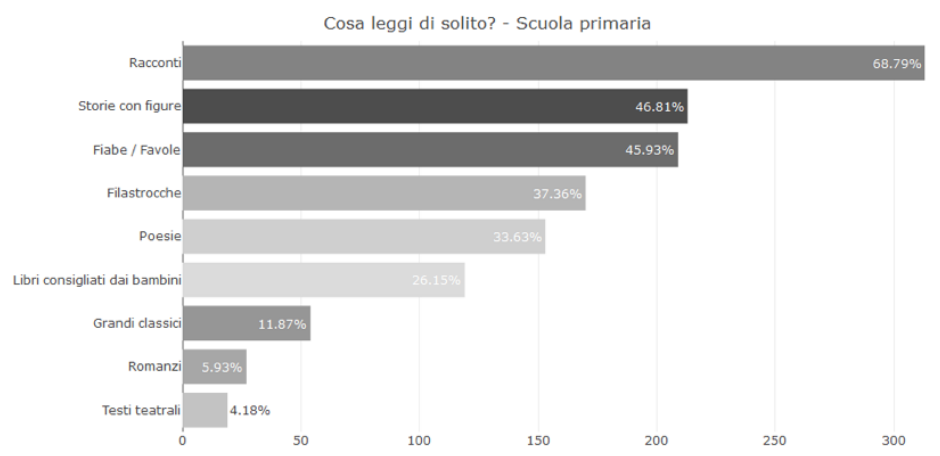

Fig. 3 - Consuetudini di lettura in classe nella scuola primaria

Se c'è un'alta presenza di studenti stranieri in classe e i generi prediletti dai docenti per la lettura con i propri alunni sono racconti e favole, quanti di questi sono tratti da libri provenienti da altre culture? Ė emerso che solo una minima parte delle unità campionarie interrogate utilizza "sempre" libri provenienti da altre culture, precisamente lo $0,5 \%$, percentuale che si alza di poco nelle unità campionarie che utilizzano "spesso" questi libri con il 7,1\%. Il $48 \%$ del campione, quindi quasi la metà, utilizza libri di altre culture "qualche volta" e il $44 \%$ ha dichiarato, invece, che non li utilizza "mai" (fig. 4). I dati sono più che eloquenti.

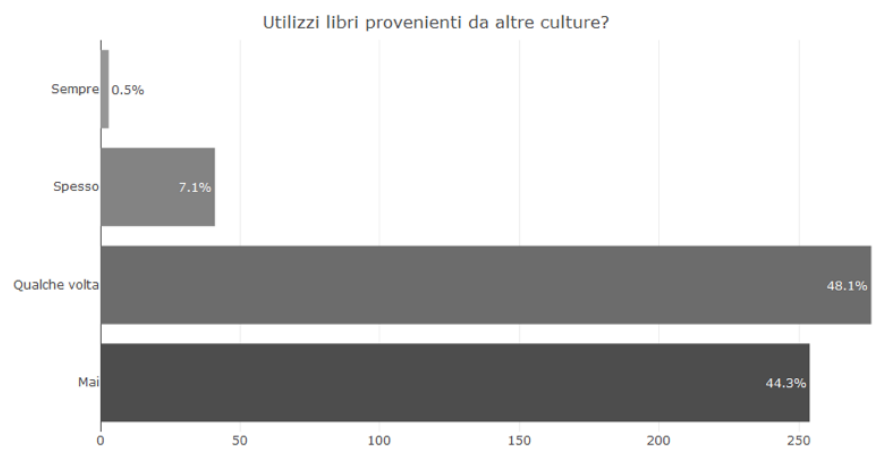

Fig. 4 - Utilizzo di libri provenienti da altre culture

Entrando nello specifico dei due ordini di scuola, per i docenti dell'infanzia la situazione resta sostanzialmente analoga: si mantengono molto basse le percentuali relative a coloro che utilizzano "sempre" o "spesso" libri provenienti da altre culture, rispettivamente lo $0 \%$ e l'1,4\%. Si abbassa al $44 \%$ la percentuale delle unità campionarie che scelgono certi testi 
"qualche volta", ma si alza a più della metà (54\%) la percentuale relativa ai docenti che non li utilizzano "mai" (fig. 5).

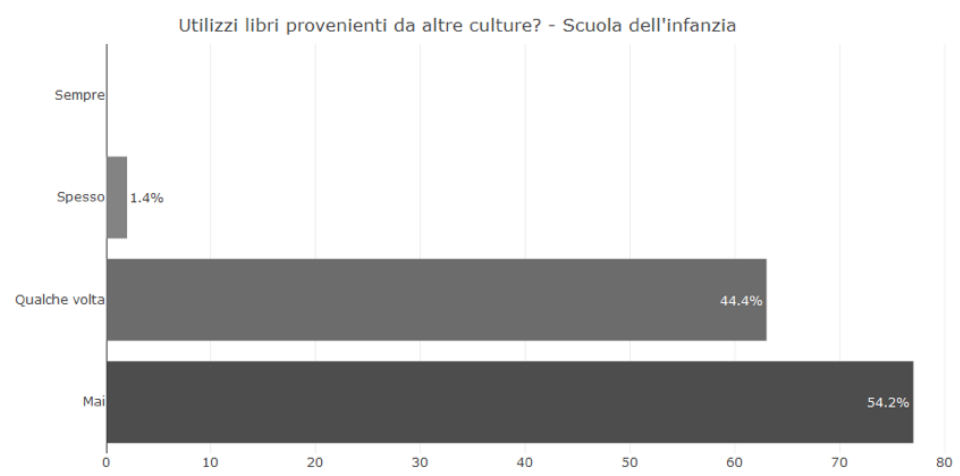

Fig. 5 - Utilizzo di libri provenienti da altre culture nella scuola dell'infanzia

Prendendo in considerazione esclusivamente i dati relativi alla scuola primaria, si riconferma il trend generale (fig. 6), ovvero una percentuale molto bassa di insegnanti che optano "sempre" per libri di altre culture $(0,7 \%)$; la percentuale di coloro che li utilizzano "spesso" si alza al $9 \%$ rispetto alla scuola dell'infanzia. Risultano analoghe le percentuali di chi li utilizza "qualche volta" (49,3\%) e "mai" (41\%).

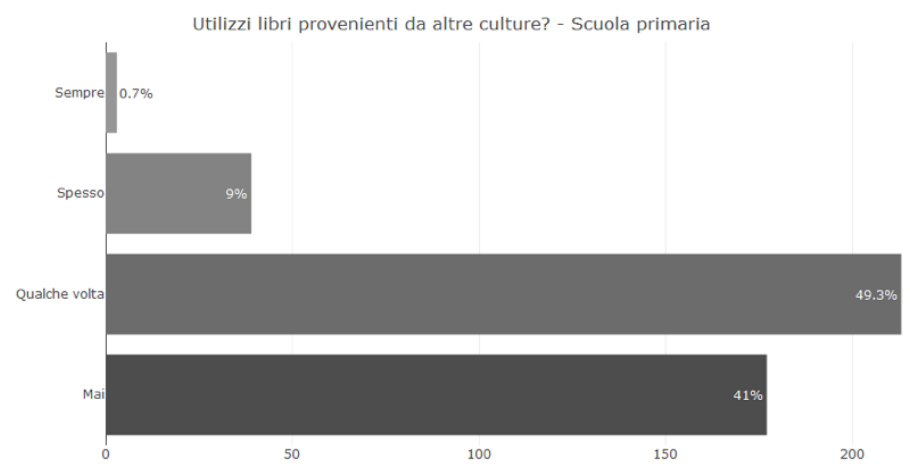

Fig. 6 - Utilizzo di libri provenienti da altre culture nella scuola primaria

Andando poi a chiedere nello specifico l'ultimo libro di altre culture letto in classe, nella maggioranza dei casi i docenti del campione non hanno fornito veri e propri titoli, ma indicazioni generiche come "fiabe africane" o "racconti arabi" (fig. 7). 


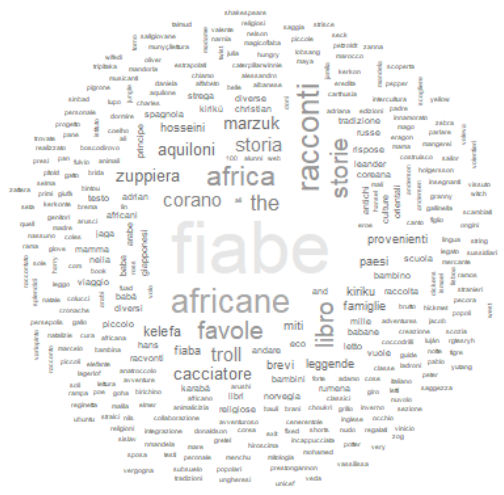

Fig. 7 - Ultimi libri di altre culture letti in classe

Solo per la scuola dell'infanzia è stato fornito qualche titolo in più, come "La zuppiera di Marzuk" e "Troll" (figg. 8-9).

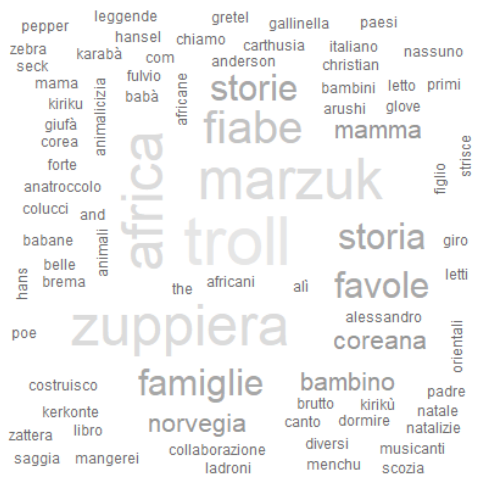

Fig. 8 - Ultimi libri di altre culture letti nella scuola dell'infanzia 


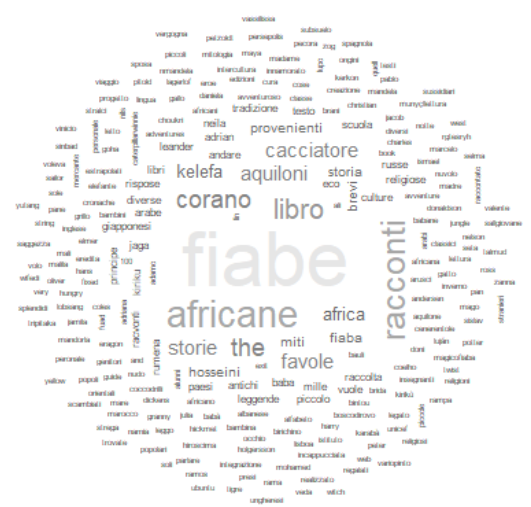

Fig. 9 - Ultimi libri letti di altre culture letti nella scuola primaria

Appare, quindi, evidente il divario tra una società già stabilmente multiculturale e una scuola che, al contrario, fatica a costruire interculturalità, intesa come dialogo e conoscenza tra le culture.

\section{Considerazioni conclusive}

A seguito dell'analisi dei dati esposti, una domanda sorge spontanea, proprio in virtù delle riflessioni teoriche riportate all'inizio di questo articolo: quali cittadini del futuro sta formando la scuola italiana? Eppure, almeno dal punto di vista normativo e legislativo, la scuola continua a ribadire l'importanza di percorrere la via dell'interculturalità, come sottolineato anche nel più recente testo normativo di riferimento emanato dal Miur, le Indicazioni nazionali per il curricolo della scuola dell'infanzia e del primo ciclo d'istruzione, datate 2012:

La scuola affianca al compito «dell'insegnare ad apprendere» quello «dell'insegnare a essere». L'obiettivo è quello di valorizzare l'unicità e la singolarità dell'identità culturale di ogni studente. La presenza di bambini e adolescenti con radici culturali diverse è un fenomeno ormai strutturale e non può più essere considerato episodico: deve trasformarsi in un'opportunità per tutti. Non basta riconoscere e conservare le diversità preesistenti, nella loro pura e semplice autonomia. Bisogna, invece, sostenere attivamente la loro interazione e la loro integrazione attraverso la conoscenza della nostra e delle altre culture (Miur, 2012, p. 10). 
Solo attraverso la conoscenza reciproca si può avviare un autentico percorso di integrazione. La lettura, in questo - specialmente la lettura ad alta voce che trova nel gruppo-classe luogo favorito di attuazione - ci appare uno strumento privilegiato, perché è attraverso le storie narrate e ascoltate che conosciamo noi stessi e gli altri. Un'esigenza, questa, dettata oggi, più che in passato, dalla realtà: flussi migratori, accoglienza, costruzioni di muri, spinte protezionistiche e reazionarie, episodi di violenza razziale, etc. riempiono ogni giorno pagine di quotidiani e ore di programmi televisivi.

Le narrazioni ci permettono di dare un senso alle esperienze che facciamo nella vita di ogni giorno e anche a esperienze che solo immaginiamo. Un senso che viene costantemente rielaborato per tenere conto delle cose nuove che capitano a noi, o intorno a noi, o dentro di noi e che è costruito in collaborazione con altre persone, reali o immaginarie. La narrazione è sempre, più o meno apertamente, un dialogo [...] Perché sottolineare la dimensione interculturale? Perché oggi, in un momento in cui viviamo fianco a fianco con persone provenienti da altre parti del mondo, la prossimità attiva i fantasmi razzisti che da secoli frequentano le società europee. Ora abbiamo bisogno di narrazioni che ci aprano al mondo in un modo incomparabilmente più profondo e sistematico di quanto avveniva quando la nostra conoscenza dell'India o dei Caraibi era affidata alle avventure di Sandokan. Che ci facciano conoscere i mondi morali di persone di altre "culture" in modo che esse non siano più estranee per noi ma le riconosciamo come nostro prossimo (Mantovani, 2013, p. 61).

Un prossimo che, forse, è più vicino a noi di quanto pensiamo. È ancora veramente possibile definire le culture del mondo "altre" da noi in maniera così netta? A tal proposito proponiamo un breve, ma significativo racconto intitolato Il piccolo mondo di Ambra, tratto da un libro scritto da maestre per altre maestre desiderose di costruire percorsi significativamente interculturali. Con la speranza che possa essere spunto di riflessione per chi questo mestiere già lo fa e per chi si prepara a farlo.

In un paese né grande né piccolo, da qualche parte in Italia, vive una bambina che si chiama Ambra, nome derivato dalla parola "anbar", che in arabo indica una pietra preziosa. Al mattino Ambra si alza presto e fa colazione con i "corn flakes" prodotti a base di mais, cereale originario del Messico. Suo padre la invita a far presto mentre beve il suo tè. Poi si veste, indossando una felpa di cotone, pianta originaria dell'India, introdotta in Europa dagli arabi a metà del secolo IX. L'etichetta della felpa dichiara: "Made in Taiwan". Ambra va scuola e risolve problemi utilizzando numeri indiani, portati in Europa dagli arabi. Durante la ricreazione mangia una banana cresciuta ai tropici e fa una partita a scacchi, gioco di antichissima origine, probabilmente indiana. Racconta poi alla sua amica Sara (che porta il nome, di origine ebraica, della santa protettrice degli zingari) come ha trascorso la domenica. 
Utilizza parole quali computer, videogame, film, judo, chimono, rispettivamente prese a prestito dall'inglese e dal giapponese. Alla mensa scolastica mangia spaghetti al pomodoro, e forse non sa che la pasta è stata inventata dai cinesi e che il pomodoro, sconosciuto in Europa fino al '500, fu importato dalle Americhe. Nel pomeriggio, l'insegnante di inglese parla di Halloween, la festa più amata dai bambini americani e Ambra si ricorda di aver sentito raccontare qualcosa di molto simile dalla sua nonna originaria della Calabria. Tornata a casa, si concede un po' di tempo davanti alla tv. Mentre guarda i "suoi" cartoni animati giapponesi e un documentario sui Masai, sgranocchia una barretta di cioccolato, ottenuta dalla lavorazione del cacao, coltivato esclusivamente nelle zone tropicali. Per sfuggire la presenza di sua sorella che si sta impiastricciando i capelli con l'hennè, polvere naturale colorata usata tradizionalmente dalle donne del Medio Oriente e del Maghreb, Ambra si rifugia nell'angolo preferito della sua stanza, su un tappeto pakistano, probabilmente fabbricato da un suo coetaneo. Fantastica di praterie, cavalli e tepee, masticando una caramella balsamica all'eucalipto, pianta di origine australiana. Nel frattempo anche papà è tornato. A tavola Ambra ascolta confusa un suo commento alle notizie del telegiornale: "Tutti questi stranieri minacciano la nostra tradizione e non hanno proprio niente da insegnarci" (Mezzini Rossi, 1997, p. 70).

\section{Riferimenti bibliografici}

Bertazzo M. (2017), Narrare a scuola: storie dentro e oltre i confini, STUDIUM EDUCATIONIS, 1: 123-136.

Besozzi E. (2006), Società, cultura, educazione, Carocci, Roma.

Caritas-Migrantes (2017), Dossier statistico Immigrazione 2016.

Frezza S. (2016), La lettura ad alta voce come strumento didattico nella Scuola Primaria per l'apprendimento delle lingue "altre", in Nanni S., a cura di, Educare oltre confine. Storie narrazioni intercultura, FrancoAngeli, Milano.

IDOS Centro Studi e Ricerche (2017), Dossier Statistico Immigrazione 2017, Edizioni IDOS Centro Studi e ricerche, Roma.

Luatti L. (2008), La via narrativa all'educazione interculturale, in Brogi V. e Mori L., a cura di, Il bambino ir-reale., Edizioni ETS, Pisa.

Mantovani G. (2013), Aprire la mente su mondi lontani e vicini: l'India, per esempio, in Batini F. e Giusti S., a cura di, Imparare dalla lettura, Loescher Editore, Torino.

Mezzini M. e Rossi C. (1997), "Gli specchi rubati" Percorsi didattici interculturali per la scuola, Meltemi, Roma.

MIUR (2007), La via italiana per la scuola interculturale e l'integrazione degli alunni stranieri. Osservatorio nazionale per l'integrazione degli alunni stranieri e per l'educazione interculturale.

MIUR (2012), Indicazioni nazionali per il curricolo della scuola dell'infanzia e del primo ciclo d'istruzione.

MIUR (2014), Linee guida per l'accoglienza e l'integrazione degli alunni stranieri.

Ongini V. (2007), "L’angolo della lettura”, Sesamo. Bambini stranieri in classe, 3: 32. 


\section{Dispersione scolastica: visioni a confronto. Indagine sulla percezione della dispersione tra studenti, insegnanti e genitori delle scuole secondarie umbre}

di Irene Dora Maria Scierri e Mariangela Nitti ${ }^{1}$

\section{Introduzione}

\subsection{Popolazione scolastica e dispersione in Umbria}

Nell'anno scolastico 2016/2017, nelle scuole secondarie statali di primo grado dell'Umbria si registrano 23.475 studenti, di cui il 75,9\% nella provincia di Perugia e il $24,1 \%$ nella provincia di Terni. Nelle scuole secondarie statali di secondo grado dello stesso territorio, si rilevano 37.234 studenti, di cui il $75,7 \%$ nella provincia di Perugia e il $24,3 \%$ nella provincia di Terni. Di questi $20.093(54,0 \%)$ sono iscritti in un liceo, $10.176(27,3 \%)$ in un istituto tecnico e 6.965 in un istituto professionale $(18,7 \%)$; dati che rispecchiano la distribuzione media nazionale per tipologia di istituti superiori (rispettivamente: $48,0 \% ; 31,5 \% ; 20,6 \%)^{2}$.

In relazione al livello di dispersione scolastica, al di là della difficoltà diffusa di stimare con precisione il fenomeno, l'Umbria rappresenta una delle regioni italiane con i livelli più bassi, sia in relazione alla percentuale di early leaving form education and training (ELET) ${ }^{3}$ che al tasso di abbandono scolastico. L'Italia, infatti, si colloca agli ultimi posti tra i 28 paesi

\footnotetext{
${ }^{1}$ Il contributo è frutto di un lavoro svolto in collaborazione, possono, tuttavia, essere attribuiti a Mariangela Nitti i paragrafi 2 e 3.6 e a Irene D.M. Scierri i restanti paragrafi (1.1, $1.2,3.1,3.2,3.3,3.4,3.5,3.7,4)$. Mariangela Nitti ha inoltre realizzato il report interattivo accessibile dal sito web dispersione.it.

${ }^{2}$ Nostre elaborazioni di dati disponibili al sito web dati.istruzione.it (i dati presenti nel sito sono aggiornati al 21/02/2017).

${ }^{3}$ In ambito europeo il tasso di ELET viene utilizzato come indicatore del fenomeno della dispersione scolastica, si riferisce ai giovani tra i 18 e i 24 anni privi di titolo o qualifica superiore alla scuola secondaria di I grado e non più in formazione. Più nel dettaglio, il nu-
} 
dell'Unione Europea (dopo di lei solo Portogallo, Romania, Spagna e Malta) con una percentuale di ELET pari al 13,8\%, contro una media EU-28 di $10,7 \%$ (Eurostat, 2017). La media nazionale risulta, pertanto, ancora al di sotto del benchmark del 10\% di ELET stabilito dalla Strategia Europa 2020. Bisogna tuttavia rilevare la forte variabilità del tasso di ELET all'interno del nostro Paese: di fronte a regioni ancora molto lontane dal target europeo, altre lo hanno già superato, tra cui l'Umbria (fig. 1).

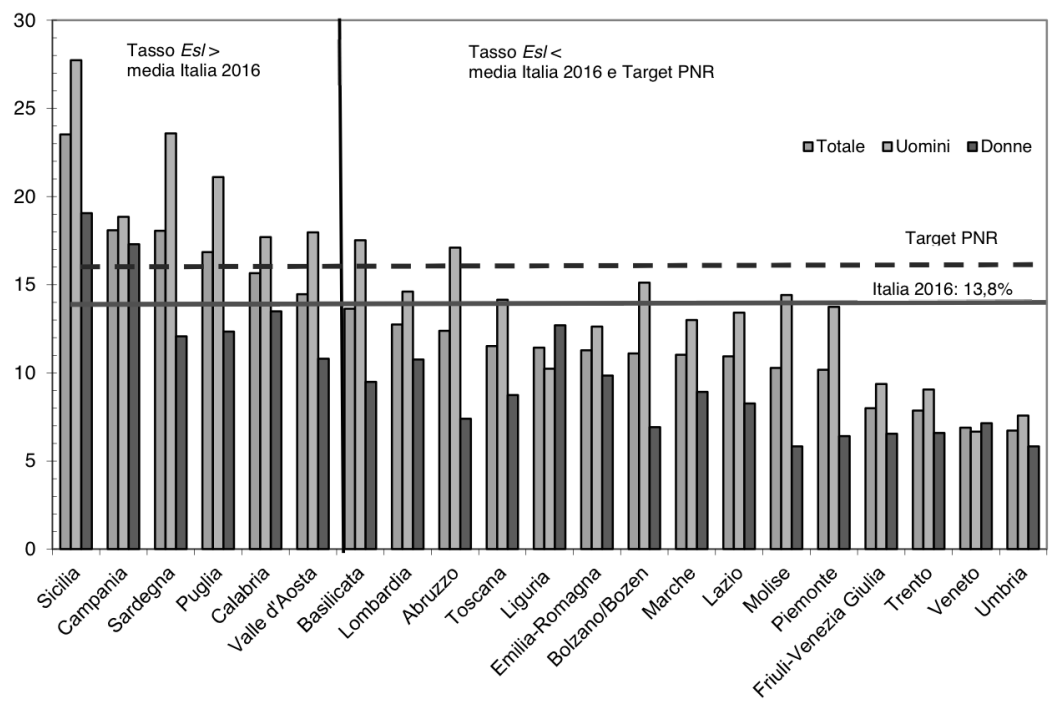

Fig. 1 - Tasso di Early school leavers in Italia nel 2016 Fonte: Istat - Rilevazione sulle forze di lavoro

L'ultimo focus sulla dispersione scolastica (Miur, 2017) - relativo, ai soli abbandoni - registra che, nell'anno scolastico 2015-2016, 14.258 studenti della scuola secondaria di primo grado, pari allo $0,8 \%$ di coloro che frequen-

meratore dell'indicatore si riferisce alle persone di età compresa tra 18 e 24 anni che soddisfano le seguenti due condizioni: (a) il livello più alto di istruzione o formazione che hanno completato è ISCED (International standard classification of education) 2011 livelli 0, 1 o 2 (livelli ISCED 1997 0, 1, 2 o 3C short) e (b) non hanno ricevuto alcuna istruzione o formazione (in altre parole né formale né non formale) nelle quattro settimane precedenti l'indagine. Il denominatore è la popolazione totale della stessa fascia di età, esclusi i rispondenti che non hanno risposto alle domande "massimo livello di istruzione o formazione completato con successo" e "partecipazione all'istruzione e alla formazione" (Eurostat, 2017). 
tavano le lezioni, hanno abbandonato gli studi prima di giugno o nel passaggio fra un anno e l'altro. Nella scuola secondaria di secondo grado l'abbandono riguarda 112.240 studenti, pari al 4,3\%. Anche in questa statistica, l'Umbria si colloca al di sotto della media nazionale sia in relazione alla scuola secondaria di primo grado - con un tasso di abbandoni pari allo $0,7 \%$ (fig. 2) - che, soprattutto, in relazione alla scuola superiore di secondo grado, in cui registra il tasso più basso, pari al 2,9\% (fig. 3).

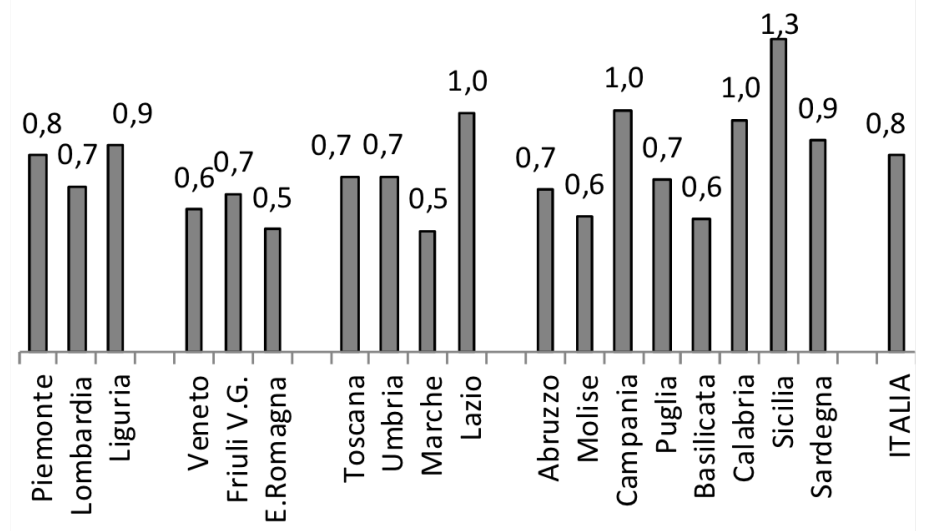

Fig. 2 - Abbandono complessivo nella scuola secondaria di I grado (\%) Fonte: MIUR - DGCASIS - Ufficio Statistica e Studi - Anagrafe Nazionale degli Studenti

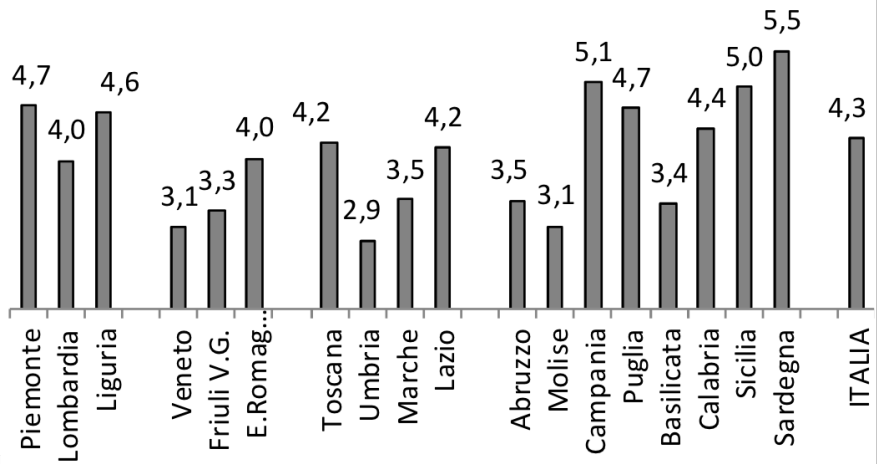

Fig. 3 - Abbandono complessivo nella scuola secondaria di II grado (\%) Fonte: MIUR - DGCASIS - Ufficio Statistica e Studi - Anagrafe Nazionale degli Studenti 
L'Umbria rappresenta dunque, all'interno del territorio italiano, un contesto peculiare per verificare quanto la problematica della dispersione scolastica sia vicina agli studenti - e ai rispettivi docenti e genitori - e quale sia la loro conoscenza e percezione del fenomeno.

\subsection{Contrastare la dispersione partendo dal punto di vista dei protagonisti}

La misurazione corretta dell'entità di un fenomeno è senza dubbio funzionale a mettere sotto i riflettori il problema e ad innescare politiche e strategie di intervento. Affinché le azioni risultino efficaci è, tuttavia, indispensabile indagare il punto di vista dei protagonisti, in primo luogo studenti ma anche docenti e genitori. Sempre più le ricerche sulla dispersione scolastica tendono, infatti, ad andare oltre le semplici analisi numeriche per aggiungere le voci e le esperienze degli studenti (Gallagher, 2002; Jeffries, Nix e Singer, 2002; Bridgeland, DiIulio e Morison, 2006; Batini, 2014; Bunting e Moshuus, 2017).

Uno degli aspetti principali legati alla comprensione della dispersione scolastica è la conoscenza dei fattori di rischio ad essa associati. La dispersione scolastica è un processo complesso, influenzato da diversi fattori, più o meno modificabili. La letteratura di riferimento ne individua generalmente quattro classi: individuali, scolastici, familiari e socioeconomici. Agire esclusivamente sui fattori di rischio individuali, ovvero quelli centrati sullo studente, non si è rivelato utile nella creazione di interventi efficaci di prevenzione (Jimerson et al., 2000; Dynarski e Gleason, 2002), al contrario i programmi che si sono dimostrati più efficaci tendono a includere diverse strategie d'azione (Glogowski, 2015). D'altro lato, se agire sui fattori di natura strutturale (come, ad esempio, lo status socioeconomico) è difficile, su altri di carattere scolastico - come la capacità di autoregolazione nell'apprendimento, il benessere nel contesto di apprendimento, la rete di relazioni con i pari e con gli insegnanti - è possibile agire, grazie a interventi efficaci da parte di docenti ed educatori (Alivernini, Manganelli e Lucidi, 2017). Tuttavia, gli studi sugli interventi di contrasto all'abbandono suggeriscono che si continua ad avere un'attenzione eccessivamente focalizzata sulle caratteristiche degli studenti piuttosto che adottare un approccio più ecologico (Davis e Dupper, 2004). In particolare, l'inclusione della relazione studente-insegnante è fondamentale sia nella comprensione delle motivazioni dell'abbandono sia nello sviluppo di interventi più efficaci a sostegno della persistenza degli allievi nel contesto scolastico (Davis e Dupper, 2004). I fattori 
legati alle pratiche disciplinari e al rapporto studenti-insegnanti influenzano, infatti, le decisioni educative degli studenti (Tyler e Lofstrom, 2009). È noto da tempo, ad esempio, il ruolo che le aspettative positive dei docenti rivestono sul successo formativo degli alunni (Rosenthal, 1973). Diverse ricerche hanno, inoltre, confermato che l'attitudine e il comportamento dei docenti, nonché la qualità della relazione insegnante-studente, hanno un impatto significativo sia sui risultati scolastici (Fredriksen e Rhodes, 2004; Fortin et al., 2006; Englund, Egeland e Collins, 2008) che sul disimpegno e l'abbandono (Pierce 1994; Bennacer, 2000; Potvin e Paradis, 2000; Christenson et al., 2001; Vellos e Vadeboncoeur, 2015; Nairz-Wirth e Feldmann, 2017).

Da parte dei docenti, la conoscenza di quali siano i fattori di rischio della dispersione e la consapevolezza del ruolo che possono avere nel contrastarla è alla base di un'efficace prevenzione. Alcuni studi hanno rilevato che le percezioni degli insegnanti sulle cause dell'abbandono tendono a concentrarsi su fattori al di fuori del loro controllo, mentre i fattori legati alla relazione studente-insegnante sono stati valutati in maniera meno forte come contributi dell'abbandono (Knesting-Lund, Reese e Boody, 2013; Bartolucci e Batini, 2016; Nairz-Wirth e Feldmann, 2017).

Le percezioni generali sui fattori chiave che influenzano l'abbandono scolastico sono significativamente diverse tra i giovani che hanno abbandonato la scuola e i rispettivi insegnanti (Obasohan e Kortering, 1999; Doll, Eslami e Walters, 2013), ma anche tra studenti, insegnanti e genitori in relazione alle cause e alle cure dell'abbandono (Bridgeland et al., 2010). Inoltre, una buona parte di insegnanti ritiene di avere poca o nessuna influenza sulle decisioni degli studenti di rimanere o abbandonare la scuola (Knesting-Lund, Reese e Boody, 2013).

Se, come accennato, diversi studi qualitativi hanno dato voce al punto di vista e alle esperienze degli studenti, le voci degli insegnanti e dei genitori sono per la maggior parte assenti. Per adottare un approccio preventivo ecologico ed efficace è necessario, per prima cosa, ascoltare la voce di tutti. In particolare, capire quale sia il livello di familiarità e di conoscenza del fenomeno della dispersione scolastica da parte di docenti, studenti e genitori è fondamentale per sviluppare interventi che includano tutti i protagonisti del contesto scolastico.

\section{Presentazione della ricerca}

Vengono qui presentati i risultati di un'indagine conoscitiva condotta dalla cattedra di Metodologia della ricerca educativa, dell'osservazione e 
della valutazione del dipartimento FISSUF dell'Università degli Studi di Perugia nell'anno accademico 2017/2018. I questionari sono stati somministrati nel mese di novembre 2017 - grazie alla collaborazione delle studentesse e degli studenti frequentati il corso ${ }^{4}$ - a gran parte degli istituti scolastici secondari di primo e secondo grado del territorio umbro 5 . Obiettivo dell'indagine è quello di indagare la percezione della dispersione scolastica delle

\begin{abstract}
${ }^{4}$ Hanno partecipato alla realizzazione della ricerca gli studenti: Rita Amato, Chiara Baldassarri, Francesco Bambini, Alessia Barbonari, Marta Belloni, Eleonora Benemio, Gaia Bettacchioli, Aurora Bianchi, David Bianconi, Cecilia Bini, Agnese Borrazza, Arianna Brunetti, Antea Busto, Margherita Calvieri, Marta Cancellieri, Dania Cardetta, Veronica Cardinali, Filippo Carinella, Lisa Carlini, Leonardo Carloni, Lucrezia Cassiani, Martina Castraberti, Martina Cattarossi, Ilaria Cavalloro, Elisa Cecchetti, Giorgia Cerquiglini, Chiara Cesarini, Francesca Cesarini, Enrica Chiffi, Giulia Chipa, Elisa Cioccoloni, Giulia Cioccoloni, Alessia Coppola, Anna Cosucci, Eleonora Crocco, Marta De Luca, Nancy De Luca, Daniele De Maio, Marika Di Corrado, Maria Chiara Di Lello, Maria Elena Emili, Teresa Espositi, Giulia Fagiolo, Sophia Farnesi, Leonardo Fasi, Giacomo Faticoni, Giovanni Ferrari, Serena Forti, Francesca Frati, Eleonora Frescura, Erica Garofoli, Nadia Gentile, Sara Giorgi, Noemi Giulivi, Alessia Giustozzi, Olivia Gola, Jessica Gurrieri, Elisa Impero, Lucrezia Laudani, Eva Liberti, Giorgia Lisetti, Mattia Loreti, Valeria Loverso, Claire Dianne Magnaye, Martina Mammoli, Giulia Mancini, Chiara Marchetta, Lucia Marconi, Eleonora Mariani, Lorenza Mariani, Marta Mariani, Chiara Mattioli, Gloria Mencacci, Carolina Mencarelli, Camilla Menichelli, Alessandra Merli, Alba Metushi, Elvira Mia, Annamaria Moio, Gessica Moretti, Elisa Morini, Elisa Mosconi, Ermira Muco, Xhensila Murati, Lucrezia Nardi, Letizia Nicastro, Giovanni Ottaviani, Halise Ozgun, Valentina Pacini, Chiara Pallotta, Alessia Panetta, Martina Paolinelli, Natascia Paradisi, Tania Paradisi, Letizia Paroli, Giorgia Pascolini, Claudia Pellicani, Anna Pennoni, Annalaura Pettirossi, Leonardo Piccotti, Giorgia Pini, Virginia Pizzichini, Giulia Pompei, Deborah Pompili, Erica Porzi, Marta Posti, Valeria Preziosi, Maria Vittoria Quintili, Alice Ramazzotti, Lucia Rampagni, Martina Rencricca, Alessia Rinaldoni, Debora Rizzi, Andrea Rogaia, Giulia Romeo, Leyla Roosta khorasani, Francesca Rossi, Michela Rossi, Luca Sabatini, Giulia Salvadori, Jessica Santini, Greta Sarri, Alessandro Sisti, Alessandra Sisto, Oksana Skrypkina, Arianna Sorrentino, Irina Spigarelli, Noemi Splendorini, Arianna Stallone, Samuele Strati, Noemi Tarani, Naomi Tinarelli, Sara Togni, Ilaria Tomassini, Marta Toro, Loredana Tortorella, Giulia Tosti, Giorgia Trabalza, Sara Troka, Vincenza Tusa, Francesca Vagnetti, Valentina Vagnozzi, Grazia Vannuzzi, Emanuela Verdura, Carlotta Vichi, Valentina Vinti, Simone Vitaletti, Maria Jole Zito.

${ }^{5}$ Hanno aderito all'indagine 12 istituti di primo grado e 19 istituti superiori: Bonazzi-Lilli (PG); Istituto comprensivo Galileo Ferraris (Spello); Istituto comprensivo 9 (PG); Istituto omnicomprensivo Dante Alighieri (Nocera Umbra, sezione Valtopina), Francesco Mari (Nocera Umbra), Istituto comprensivo B. Bonfigli (Corciano), Alighieri-Pascoli (Città di Castello), Carducci-Purgotti (PG), Frate Francesco (Assisi), Istituto comprensivo 5 (Foligno), Ugo Foscolo (PG), Galeazzo Alessi (Assisi), Centro internazionale Maria Montessori (PG), FrezziBeata Angela (Foligno), IIS Cassatta Gattapone (Gubbio), IIS Cavour Marconi Pascal (PG), IIS Giodano Bruno (PG), IIS Leonardo Da Vinci (Umbertide), IIS Marco Polo-Bonghi (Assisi), Istituto alberghiero G. De Carolis (Spoleto), Istituto omnicomprensivo Salvatorelli-Moneta (Marsciano), ITE Scarpellini (Foligno), ITET Capitini (PG), ITT Leonardo Da Vinci (Foligno), ITTS A. Volta (PG), Liceo delle scienze umane Don Gino Sigismondi (Nocera
\end{abstract}


categorie di soggetti più direttamente interessanti al fenomeno: studenti, docenti e genitori. In particolare, si è scelto di rilevare la percezione della dispersione tra gli studenti frequentanti le classi seconde e terze delle scuole di primo grado e le classi prime e seconde del secondo grado di istruzione. A questa tipologia di rispondenti si aggiungono i rispettivi insegnanti e genitori.

La rilevazione è stata condotta attraverso l'uso di tre questionari, differenziati per tipologia di rispondenti, messi a punto dal gruppo di ricerca con la partecipazione delle studentesse e degli studenti. Al di là di lievi differenze, prevalentemente linguistiche, i tre strumenti di rilevazione presentano la stessa struttura, finalizzata ad indagare alcune dimensioni fondamentali connesse al fenomeno della dispersione scolastica: 1) esperienza diretta della dispersione; 2) conoscenza del fenomeno; 3) fattori di rischio; 4) misure di prevenzione e contrasto; 5) stima della dispersione; 6) esperienza di formazione specifica sulla dispersione (solo docenti).

La numerosità delle variabili rilevate non consente di riportare in questa sede un'analisi dettagliata dei risultati. A tale scopo, il report "La Percezione della Dispersione Scolastica nelle Scuole Umbre", accessibile dal sito web indagineumbria.dispersione.it, consente una visualizzazione interattiva dei dati e delle elaborazioni statistiche. Il report, riproducendo la struttura del questionario, consente, per ciascuna delle dimensioni indagate, di affinare il dettaglio delle analisi a diversi livelli (per tipo di rispondente, per caratteristiche del rispondente, per istituto scolastico, ecc.).

Di seguito vengono presentati i dati generali e sottolineate analogie e differenze - tra le risposte di studenti, docenti e genitori - che appaiono di maggiore rilievo.

\section{Risultati}

\subsection{I rispondenti}

Sono stati raggiunti nel complesso 1713 studenti: 861 femmine $(50,3 \%)$, 812 maschi (47,4\%), 40 di sesso "non specificato" (2,3\%); 594 appartenenti alla secondaria di I grado (34,7\%), 1119 alla secondaria di II grado (65,3\%).

Umbra), Liceo A. Pieralli (PG), Liceo artistico A. Magnini (Deruta), Liceo Donatelli (TR), Liceo scientifico Galileo Galilei (PG), Liceo scientifico Raffaele Casimiri (Gualdo Tadino). 
Gli studenti rispondenti frequentano, dunque, prevalentemente gli istituti superiori. Nello specifico: 518 un liceo $(46,3 \%), 423$ un istituto tecnico $(37,8 \%)$ e 178 un istituto professionale (15,9\%) (fig. 4$)$.

Tab. 1 - Caratteristiche anagrafiche delle tre tipologie di rispondenti

\begin{tabular}{cccc}
\hline & Femmine (\%) & Maschi (\%) & Età media (anni) \\
\hline Studenti & 50,3 & 47,4 & $12,8^{*} ; 14,7^{* *}$ \\
Docenti & 75,7 & 24,3 & 48,0 \\
Genitori & 72,1 & 27,3 & 46,1 \\
\hline
\end{tabular}

* I grado; ** II grado

Per quanto riguarda i docenti, hanno risposto al questionario 367 insegnanti: 278 femmine $(75,7 \%)$ e 89 maschi $(24,3 \%) ; 125$ insegnano in una scuola di primo grado $(34,1 \%), 195$ in una scuola di secondo grado $(53,1 \%)$, 47 non hanno specificato il livello di scuola $(12,8 \%)$. Oltre la metà dei docenti intervistati insegna da almeno 16 anni (fig. 5).

Livello di scuola

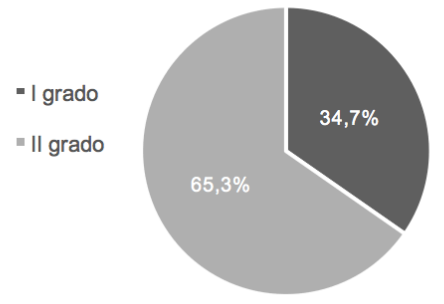

Tipologia di scuola di Il grado

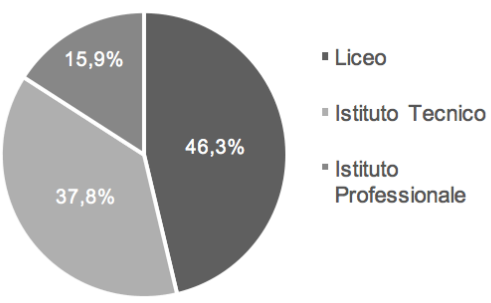

Fig. 4 - Distribuzione degli studenti per livello e tipologia di scuola

Livello di scuola

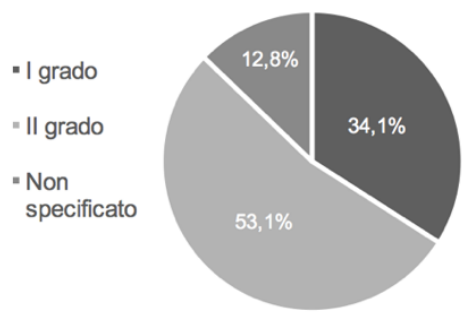

Anzianità di servizio

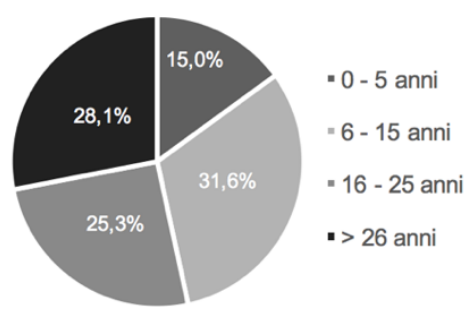

Fig. 5 - Distribuzione dei docenti per livello di scuola e anzianità di servizio 
La tipologia di rispondenti meno raggiunta è stata quella dei genitori, ovvero 183 soggetti, di cui 132 femmine (72,1\%), 50 maschi $(27,3 \%), 1$ di sesso non specificato $(0,6 \%)$. Il titolo di studi prevalente è il diploma di maturità $(50,3 \%)$, seguito dal diploma universitario/laurea $(28,4 \%)$, licenza media o avviamento $(13,7 \%)$, titolo post-laurea $(7,1 \%)$, altro titolo $(0,5 \%)$.

È interessante notare che solo il 4,9\% dei genitori che hanno risposto al questionario ha figli che hanno subito bocciature e nessuno ha figli che hanno abbandonato la scuola. Possiamo ragionevolmente supporre che i genitori che hanno risposto al questionario siano quelli più vicini e interessati alla vita scolastica in senso lato dei propri figli, con cui hanno probabilmente un rapporto non problematico e comunicativo (erano infatti i figli il tramite tra la ricerca e i genitori) e, come emerso dalle risposte sopradescritte, poco vicini alla problematica della dispersione scolastica.

\subsection{L'esperienza diretta della dispersione}

La prima sezione del questionario ha lo scopo di indagare quanto studenti, docenti e genitori abbiano una esperienza diretta della problematica. A tale scopo è stato chiesto a tutte e tre le tipologie di rispondenti se avessero conoscenza di ragazze o ragazzi 1) intenzionati ad abbandonare la scuola e 2) che hanno abbandonato la scuola.

Alla domanda Hai mai conosciuto ragazzi intenzionati ad abbandonare la scuola? sono soprattutto docenti $(78,5 \%)$ e studenti $(56,1 \%)$ a dare una risposta affermativa, mentre il $78,7 \%$ dei genitori dichiara di non conoscere nessun soggetto intenzionato ad abbandonare la scuola (fig. 6). Questo conferma l'ipotesi sulla tipologia di genitori che abbiamo raggiunto, ovvero poco vicina al problema della dispersione, non presente né all'interno della propria famiglia - ricordiamo che non ci sono genitori di figli che hanno abbandonato e solo una percentuale residua ha figli che hanno subito bocciature - né, come emerge da questa domanda, nel loro contesto relazionale.

In relazione a studenti e insegnanti è interessante rilevare, per questa come per altre dimensioni indagate, la differenza tra chi frequenta o insegna negli istituti di primo grado rispetto al secondo grado. Sono infatti principalmente i docenti che insegnano nella scuola secondaria di secondo grado a conoscere studentesse e studenti intenzionati ad abbandonare: 1 ' $86,7 \%$ contro il $68,0 \%$ dei docenti della secondaria di primo grado. 

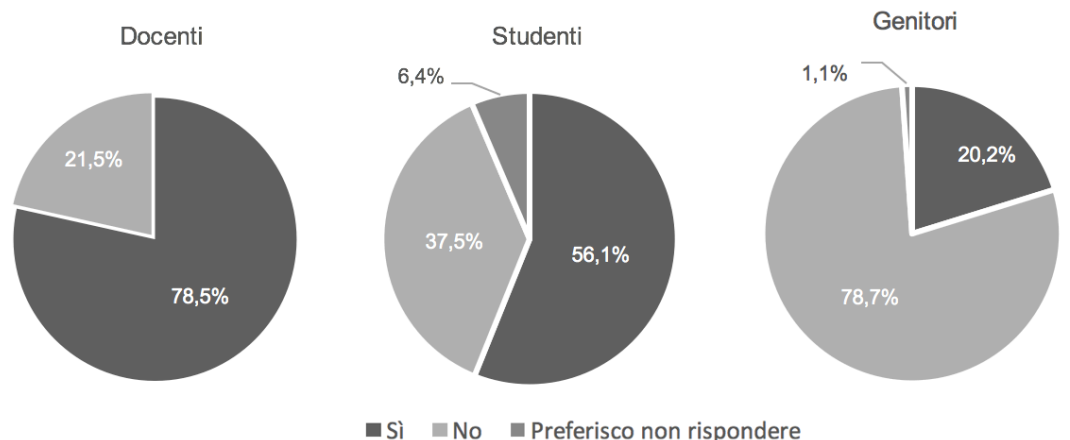

घì $\quad$ No $\quad$ Preferisco non rispondere

Fig. 6 - Conoscenza diretta di soggetti intenzionati ad abbandonare la scuola

Analoga situazione per gli studenti: il $61,0 \%$ di coloro che frequentano un istituto superiore risponde "sì" alla domanda, contro il 46,6\% degli studenti che frequentano la secondaria inferiore.

Questa maggiore vicinanza alla realtà della dispersione negli istituti superiori rispecchia l'effettiva maggiore incidenza del problema entro questo livello di scuola, in particolar modo nei primi due anni del secondo ciclo di istruzione. Inoltre, approfondendo le risposte degli studenti delle superiori per tipologia di scuola, è possibile notare che, anche in questo caso, l'esperienza diretta rispecchia l'incidenza della dispersione rilevata a livello nazionale: maggiore negli istituti professionali, minore negli istituti tecnici e ancora minore nei licei. Il 79,2\% degli studenti frequentanti un istituto professionale, infatti, dichiara di conoscere qualcuno intenzionato ad abbandonare la scuola, circa 24 punti percentuali in più rispetto agli studenti liceali (fig. 7).

Alla domanda Conosci ragazzi che hanno abbandonato la scuola? sono di nuovo i docenti e gli studenti a rispondere prevalentemente in maniera positiva: rispettivamente il $72,8 \%$ e il $62,1 \%$. La maggioranza dei genitori, al contrario, non conosce soggetti che hanno abbandonato la scuola (risponde "no" il 59,6\%) (fig. 8).

Approfondendo la distinzione tra primo e secondo grado, è possibile nuovamente evidenziare una maggiore vicinanza all'esperienza di abbandono nei docenti e negli studenti delle scuole superiori di secondo grado. L'83,1\% degli insegnanti di scuola di secondo grado risponde, infatti, "sì" alla domanda, contro il 59,2\% dei loro colleghi di primo grado. Per quanto riguarda 
gli studenti, sono il $69,6 \%$ di coloro che frequentano un istituto superiore a dichiarare di conoscere coetanei che hanno già abbandonato la scuola, contro il $47,8 \%$ degli studenti di primo grado.

In relazione agli studenti della secondaria di secondo grado, ben 1' $83,7 \%$ di coloro che frequentano un istituto professionale dichiara di conoscere ragazzi che hanno abbandonato la scuola, la percentuale diminuisce progressivamente in relazione agli studenti degli istituti tecnici $(70,4 \%)$ e dei licei (64,1\%) (fig. 9).

\section{Hai mai conosciuto ragazzi intenzionati ad abbandonare la scuola?}

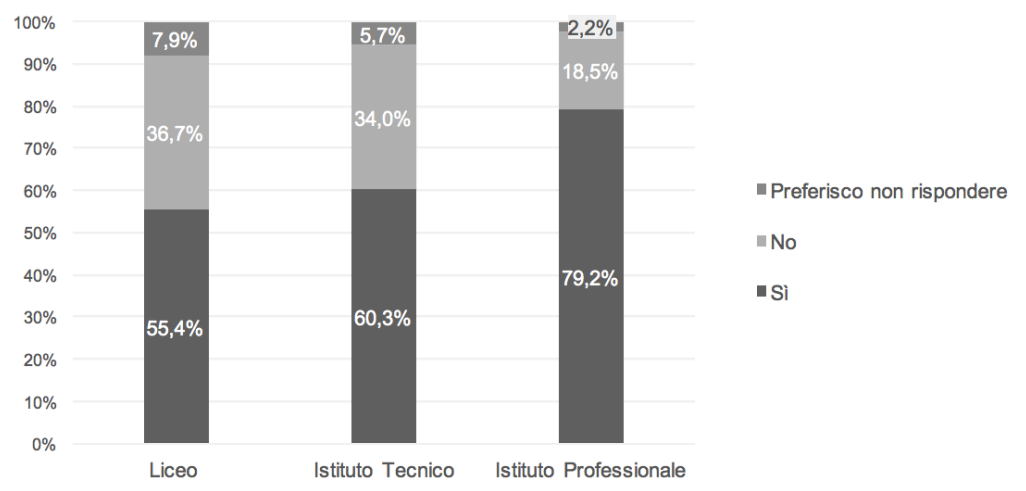

Fig. 7 - Conoscenza diretta di soggetti intenzionati ad abbandonare la scuola (studenti secondaria di II grado)

La terza domanda di questa sezione è stata rivolta esclusivamente agli studenti, con l'intenzione di rilevare quanti di essi avessero pensato di abbandonare la scuola. Tra gli studenti della scuola di primo grado ha pensato di abbandonare la scuola il $26,3 \%$ contro il $35,6 \%$ degli studenti della secondaria di secondo grado (fig. 10). 

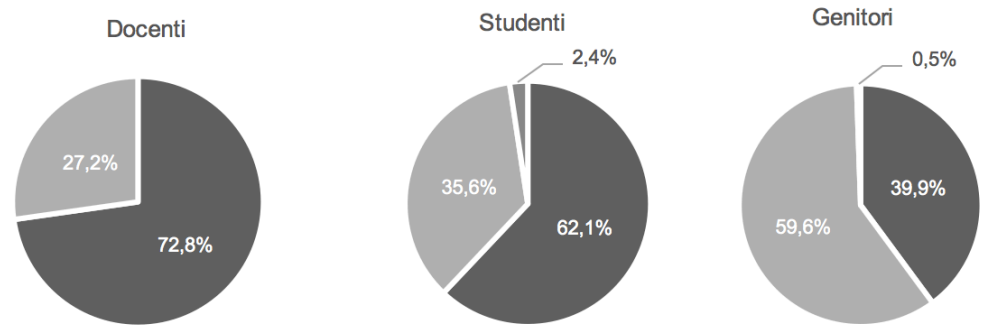

- Sì No $\quad$ Preferisco non rispondere

Fig. 8 - Conoscenza diretta di soggetti che hanno abbandonato la scuola

\section{Conosci ragazzi che hanno abbandonato la scuola?}

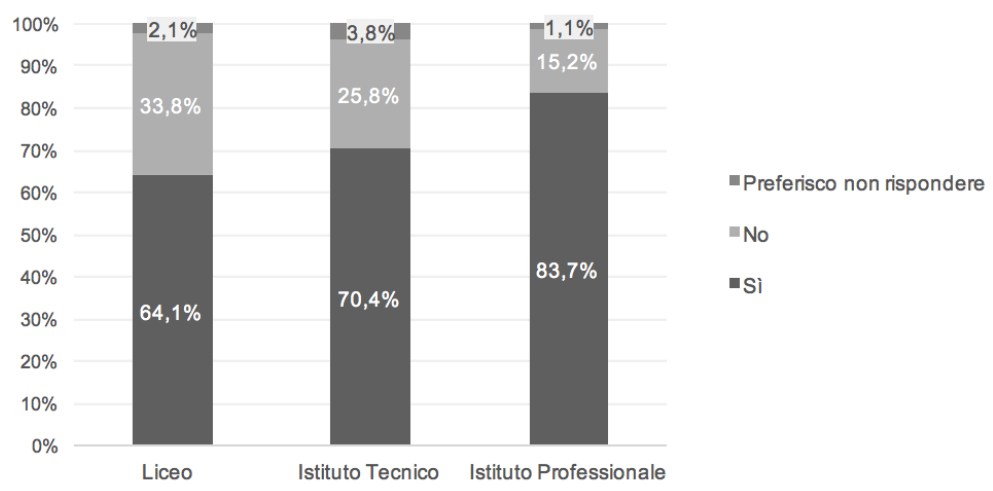

Fig. 9 - Conoscenza diretta di soggetti che hanno abbandonato la scuola (studenti secondaria di (I grado)

Se analizziamo la distribuzione delle risposte fornite dagli studenti delle superiori per tipologia di istituto, quasi la metà degli studenti frequentanti un istituto professionale ha pensato di abbandonare la scuola $(48,8 \%)$, di cui il $13,5 \%$ dichiara di pensarci spesso. Le percentuali relative al pensiero di abbandono diminuiscono tra $\mathrm{i}$ rispondenti appartenenti a un istituto tecnico $(36,6 \%)$ e a un liceo $(30,4 \%)$ (fig. 11). I dati mostrano, complessivamente, che circa uno studente delle superiori su tre ha pensato di abbandonare la scuola. 
In sintesi, in relazione all'esperienza diretta del fenomeno della dispersione scolastica, quello che emerge è che docenti e studenti hanno un'alta familiarità con questa realtà: sette docenti e sei studenti su dieci, infatti, hanno conoscenza di ragazze o ragazzi che hanno abbandonato la scuola.

\section{Personalmente, hai mai pensato di abbandonare la scuola?}

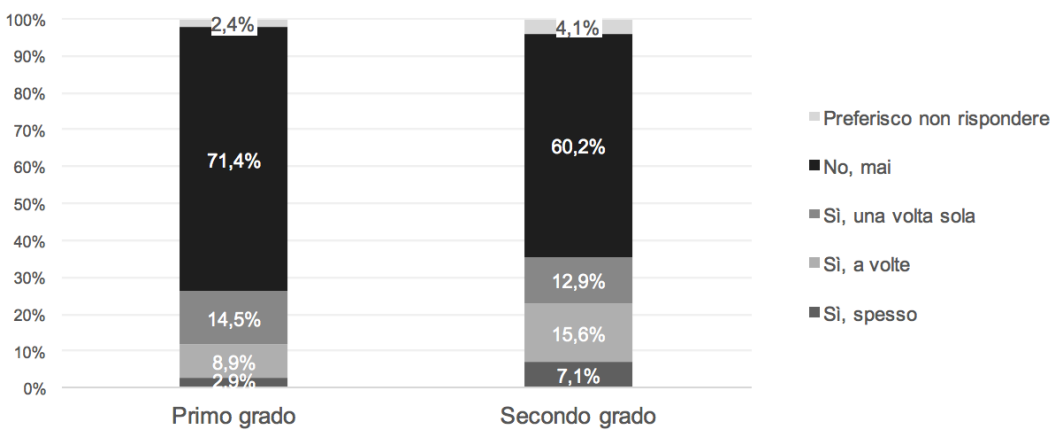

Fig. 10 - Pensiero di abbandono scolastico degli studenti per livello di scuola

Personalmente, hai mai pensato di abbandonare la scuola?

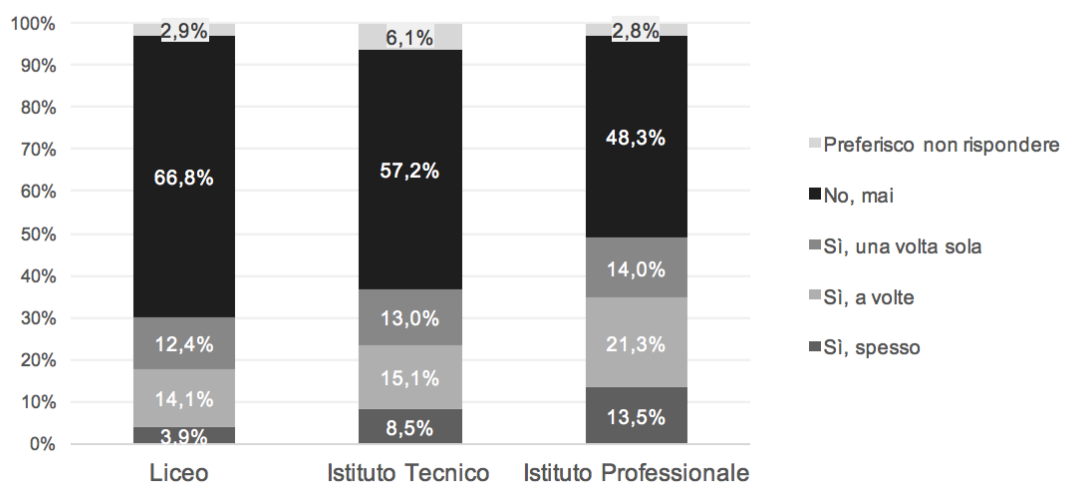

Fig. 11 - Pensiero di abbandono scolastico degli studenti per tipologia di istituto superiore 


\subsection{Conoscenza del fenomeno della dispersione}

Come appena rilevato, l'esperienza diretta della dispersione scolastica è molto diffusa nel territorio in esame. È, dunque, importante capire quanto e cosa si conosce di un fenomeno che appare così vicino alla realtà scolastica di docenti e studenti. Indagare il livello di conoscenza della dispersione scolastica è lo scopo della seconda sezione del questionario.

La prima domanda - Hai mai sentito parlare di dispersione scolastica? - è stata posta esclusivamente a studenti e genitori (dando per scontato che, almeno l'esistenza della problematica, rientri tra le conoscenze professionali di base di ogni docente). A questa domanda ha risposto "no" il 76,4\% degli studenti (fig. 12). Ricordiamo che il 62,1\% degli stessi studenti dichiara di avere conoscenza di soggetti che hanno abbandonato la scuola. Emerge chiaramente una discrepanza tra la conoscenza reale di persone che abbandonano e le informazioni che gli studenti posseggono sul fenomeno. Tra i genitori, come è logico aspettarsi, la percentuale di coloro che hanno almeno sentito parlare di dispersione è più alta (il 61,2\% risponde "sì").

\section{Hai mai sentito parlare di dispersione scolastica?}

Studenti

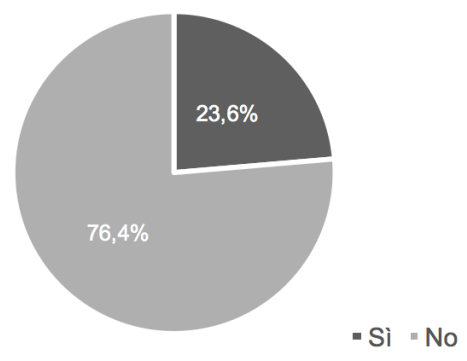

Genitori

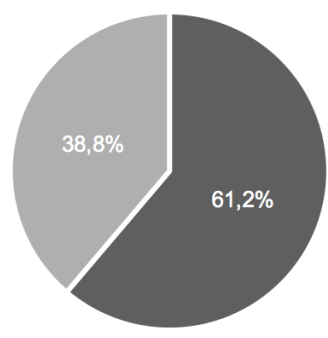

Fig. 12 - Conoscenza del fenomeno della dispersione scolastica

La seconda domanda di questa sezione intende andare oltre la semplice conoscenza superficiale del fenomeno (l'averne sentito parlare), mirando a scoprire a quali categorie di studenti viene associato il termine "dispersione scolastica". In letteratura la dispersione viene riferita a soggetti che hanno abbandonato formalmente la scuola, i bocciati e coloro che, seppur regolar- 
mente iscritti, non frequentano le lezioni. Sono state pertanto individuate dodici categorie di soggetti, ed è stato chiesto ai rispondenti di valutare - su una scala likert a tre passi (per niente, in parte, molto) - quanto ognuna delle categorie fosse attinente all'espressione "dispersione scolastica".

Dalle risposte fornite dagli studenti (fig. 13), è possibile rilevare come le prime tre categorie che hanno ottenuto l'associazione più forte col fenomeno della dispersione sono, correttamente, i "ritirati" (ovvero coloro che abbandonano formalmente gli studi), i "non frequentanti" e i "bocciati". "Ritirati" e "non frequentanti" si situano ugualmente tra le prime due categorie riconosciute come associate alla dispersione da parte di docenti e genitori (figg. 14, 15). Tuttavia, più dei "bocciati" $i$ docenti associano alla dispersione $i$ soggetti "a cui non piace la scuola" e i genitori coloro "non interessati alle discipline".

In relazione alle categorie di soggetti che rientrano nel concetto di dispersione, ciò che sorprende maggiormente sono le risposte dei docenti, che dovrebbero possedere informazioni adeguate su una delle problematiche principali della realtà scolastica. Se, infatti analizziamo le percentuali di risposta che hanno ottenuto le tre categorie che classicamente rientrano nella definizione di "dispersione scolastica", rileviamo che, ad esempio, solo quattro docenti su dieci ritengono che la categoria "bocciati" abbia molto a che fare con la dispersione. La categoria associata in maniera più forte alla dispersione è quella dei "ritirati", ciò rileva che, come spesso accade, si tende a far coincidere abbandono e dispersione, non riconoscendo come dispersione anche il numero di bocciati e di non frequentanti.

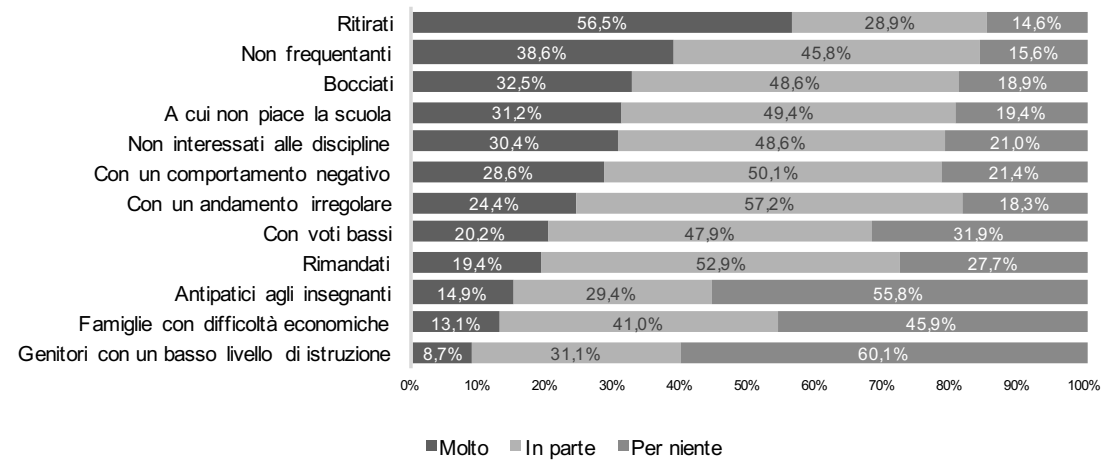

Fig. 13 - Valutazione dell'associazione tra determinate categorie di soggetti e dispersione scolastica (studenti) 


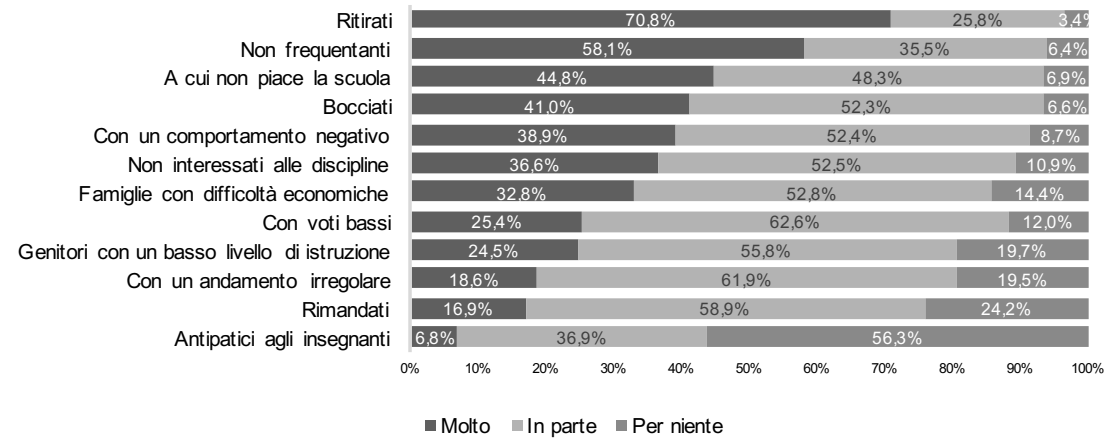

Fig. 14 - Valutazione della associazione tra determinate categorie di soggetti e dispersione scolastica (docenti)

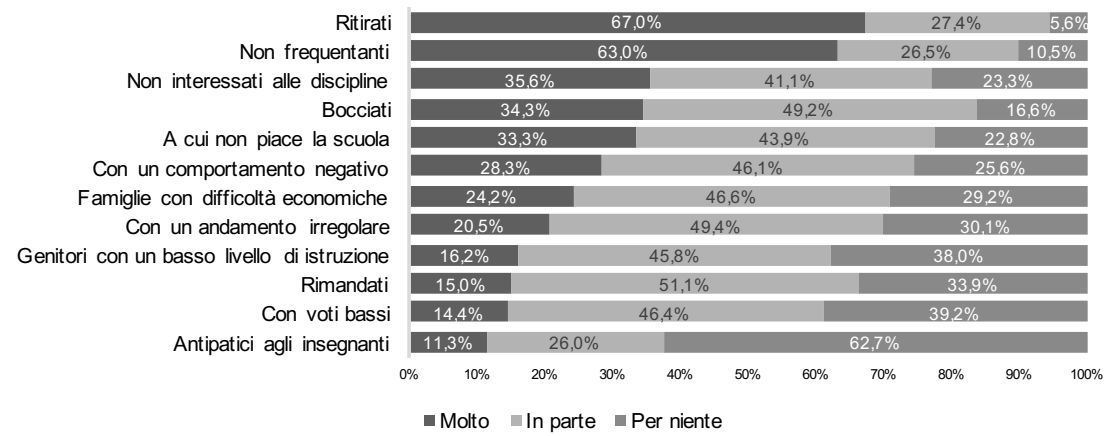

Fig. 15 - Valutazione della associazione tra determinate categorie di soggetti e dispersione scolastica (genitori)

\subsection{Fattori di rischio}

La terza sezione del questionario intende valutare la percezione dei fattori di rischio che possono essere associati alla dispersione scolastica. Questa sezione è stata presentata dopo aver fornito una definizione del concetto in esame, al fine di ottenere una valutazione dei fattori di rischio alla luce di una concezione non distorta della dispersione. 
In base alla letteratura sull' argomento sono stati individuati trenta fattori di rischio, appartenenti a quattro tipologie: "studio", "caratteristiche personali", "famiglia e amici" e "scuola". È stato quindi chiesto ai rispondenti di esprimere quanto - su una scala likert a 5 passi (per niente, poco, abbastanza, molto, moltissimo) - i fattori presentati possano influire sulla dispersione scolastica. Nelle figure 16-18 sono riportati i primi dieci fattori per ogni categoria di rispondente, in relazione alla maggiore influenza loro attribuita sulla dispersione. Soffermando l'attenzione sui primi tre fattori, possiamo notare come, se da un lato gli studenti attribuiscono maggiore influenza ai fattori connessi esclusivamente allo studio ("risultati deludenti", "scarsa motivazione connessa allo studio" e "difficoltà nello studio"), dall'altro, i docenti tendono ad estendere la tipologia dei fattori alle caratteristiche personali dello studente ("essere poco motivato" e "avere una bassa autostima") mentre i genitori al contesto familiare e amicale dello studente ("ambiente familiare che non valorizza i risultati scolastici" e "amicizia con coetanei poco interessati alla scuola"). Quello che qui interessa sottolineare è che studenti, docenti e genitori sono concordi nell'individuare la scarsa motivazione allo studio come uno dei principali fattori di rischio connessi alla dispersione.

${ }^{6}$ Di seguito i 30 fattori di rischio presentati nei questionari, raggruppati per tipologia. Studio: risultati deludenti a scuola; scarsa motivazione allo studio; difficoltà nello studio; difficoltà linguistiche (nel caso di studenti stranieri). Caratteristiche personali: essere vittima di derisione da parte di altri studenti; avere rapporti conflittuali con gli insegnanti; essere già stati bocciati; essere poco motivato; bassa autostima; avere interessi extrascolastici; avere bassi livelli di competenze di base; essere intimidito dagli insegnanti; avere problemi di salute/essere portatore di disabilità; difficoltà a fare nuove amicizie. Scuola: insegnanti poco disponibili; insegnanti troppo severi; quantità di lavoro richiesta; fissità della valutazione; rapporto difficile tra insegnante e studente; mancanza di supporto individuale agli studenti; prevalenza di lezioni frontali, tradizionali o troppo teoriche; classi numerose; mancanza di attività extra-scolastiche; istituto troppo grande. Famiglia e amici: ambiente familiare che non valorizza i risultati scolastici; amicizie con coetanei poco interessati alla scuola; ambiente familiare troppo interessato ai risultati scolastici; necessità di lavorare; difficoltà economiche della famiglia; bassa istruzione dei genitori. 


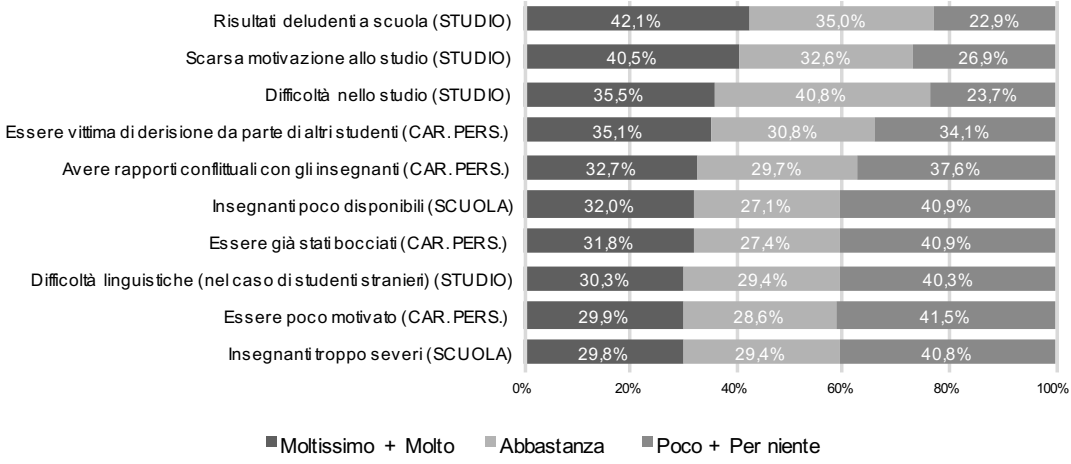

Fig.16 - Valutazione dell'incidenza dei fattori di rischio sulla dispersione (studenti)

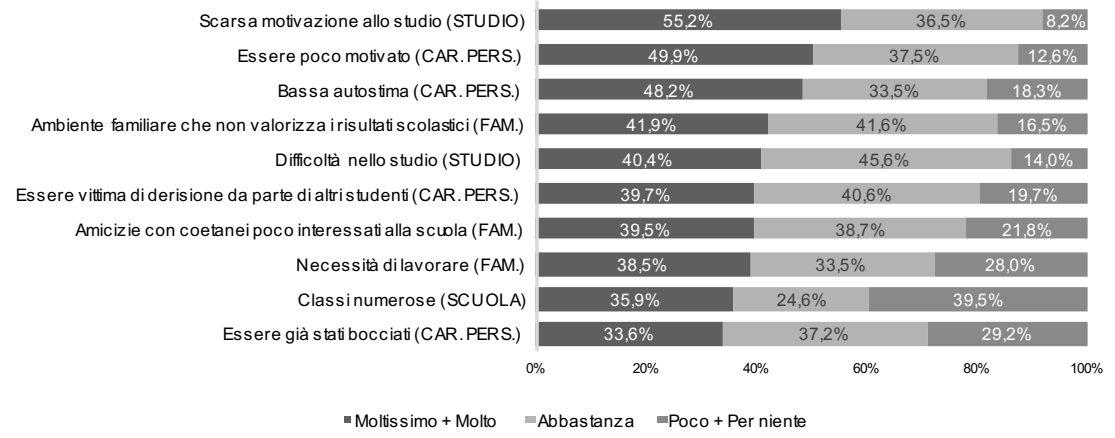

Fig.17 - Valutazione dellincidenza dei fattori di rischio sulla dispersione (docenti)

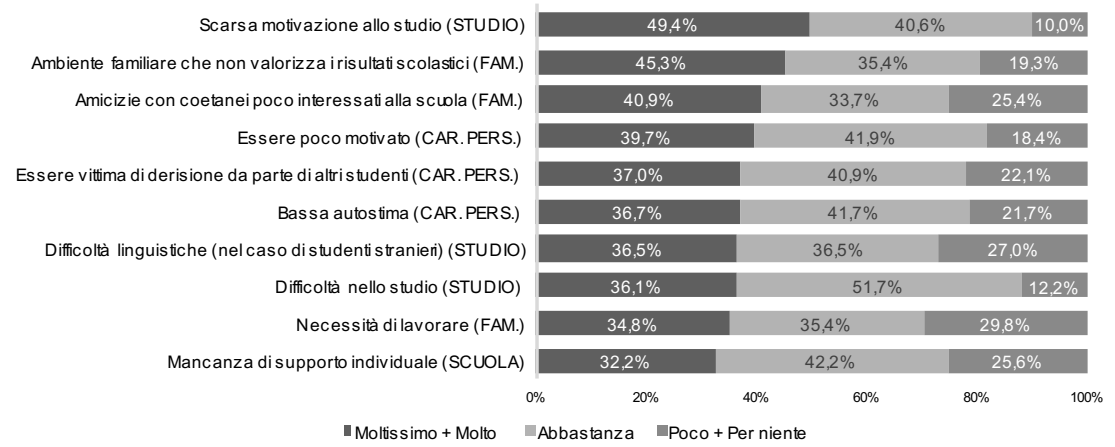

Fig. 18 - Valutazione dellincidenza dei fattori di rischio sulla dispersione (genitori) 


\subsection{Misure di prevenzione e contrasto}

La quarta sezione del questionario riguarda, in primo luogo, le misure di prevenzione e contrasto alla dispersione riconosciute efficaci da studenti, docenti e genitori e, in secondo luogo, realizzate all'interno delle scuole dei soggetti partecipanti. Sono state a tale scopo individuate dieci azioni connesse alla prevenzione della dispersione ed è stato chiesto ai rispondenti di valutare - su una scala likert a 5 passi (per niente, poco, abbastanza, molto e moltissimo) - quanto ciascuna di esse sia efficace per prevenire o contrastare la dispersione.

Le figure 19-21 mostrano le risposte di studenti, docenti e genitori in relazione alla maggiore efficacia riconosciuta. Soffermando l'attenzione sulle tre azioni ritenute maggiormente efficaci, è possibile notare un sostanziale accordo tra studenti e genitori nel riconoscere l'efficacia di: lezioni innovative e coinvolgenti, strumenti didattici per favorire l'attivazione degli studenti ed esperienze concrete di apprendimento. Differiscono le risposte dei docenti che riconoscono come azioni maggiormente efficaci il tutoraggio, $\mathrm{i}$ corsi di sostegno individuale e i corsi per lo sviluppo personale e sociale. $\mathrm{Se}$, quindi, studenti e genitori sono concordi nel riconoscere come maggiormente efficace agire sulla didattica, nella direzione di un maggiore coinvolgimento degli studenti, i docenti ritengono che possa dare risultati più efficaci agire sullo studente, nei termini di un maggiore supporto individuale.

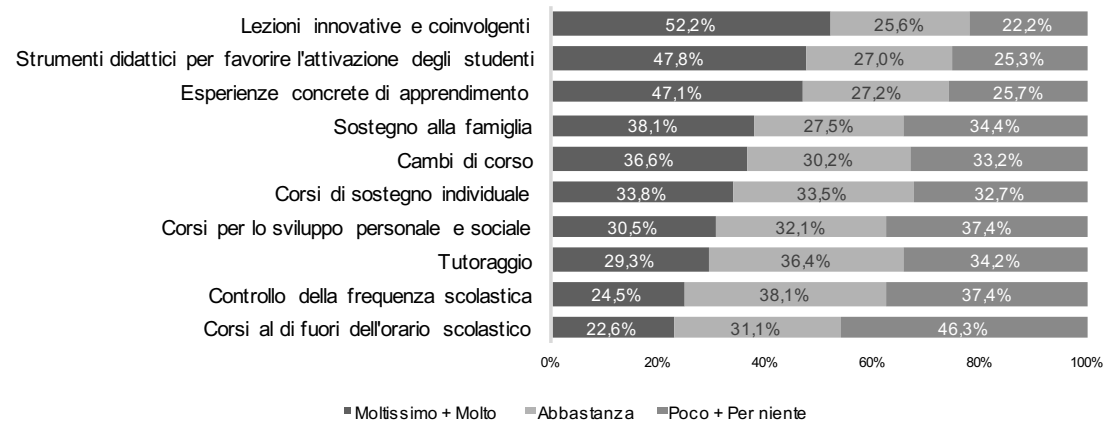

Fig. 19 - Valutazione di efficacia delle misure di prevenzione e contrasto alla dispersione (studenti) 


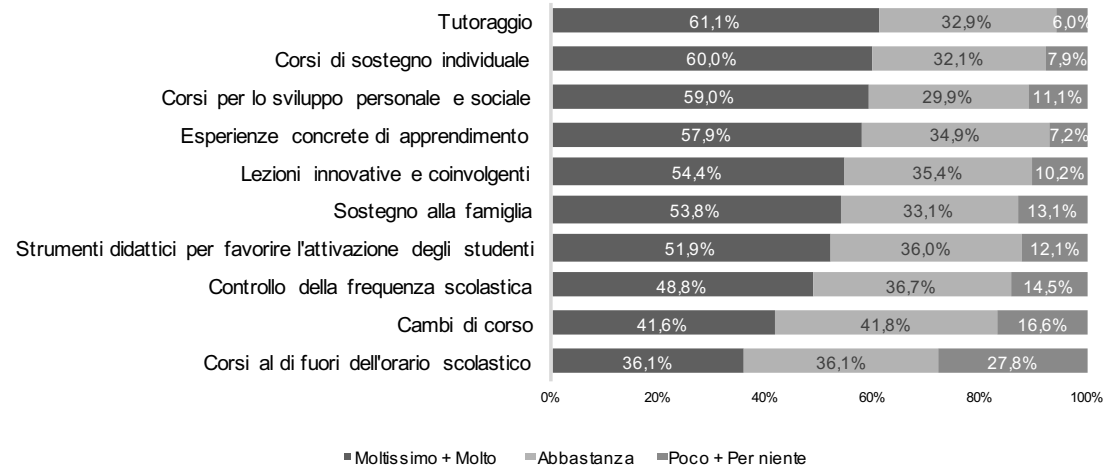

Fig. 20 - Valutazione di efficacia delle misure di prevenzione e contrasto alla dispersione (docenti)

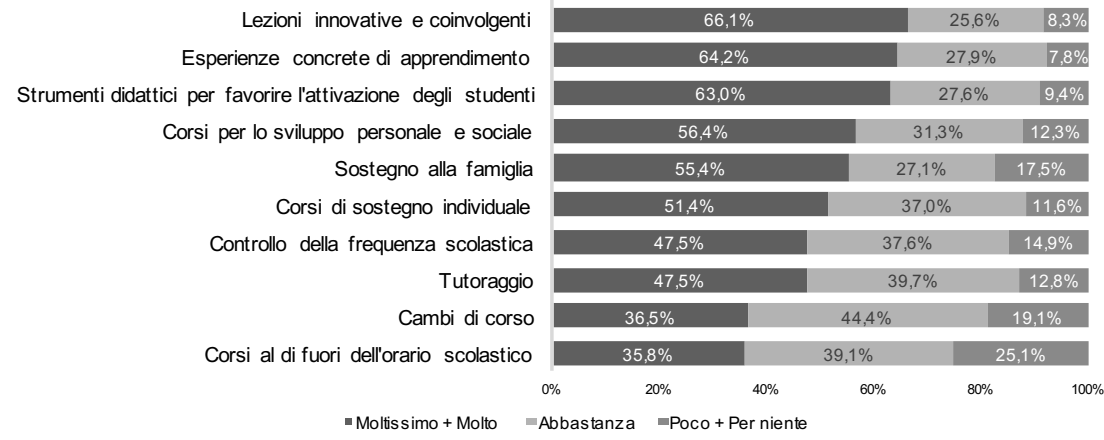

Fig. 21 - Valutazione di efficacia delle misure di prevenzione e contrasto alla dispersione (genitori)

In seconda istanza è stato chiesto a studenti e docenti quali azioni, tra quelle elencate, fossero già realizzate all'interno della propria scuola. Confrontando le risposte di studenti e docenti è possibile notare come l'azione principale messa in atto sia il controllo della frequenza scolastica (figg. 22, 23); azione che - dal punto di vista degli studenti - rientra tra quelle meno utili, insieme ai corsi al di fuori dell'orario scolastico (fig. 19). È, infine, interessante notare come ci sia una diversa percezione tra docenti e studenti relativamente alla realizzazione di lezioni innovative e coinvolgenti, esperienze concrete di apprendimento e strumenti didattici per favorire l'attivazione degli studenti. Oltre il $70 \%$ dei docenti ritiene, infatti, di realizzare nella propria scuola tali approcci didattici (fig. 23), tuttavia, solo circa la metà degli studenti ritiene che questi approcci vengano realizzati (fig. 22). 


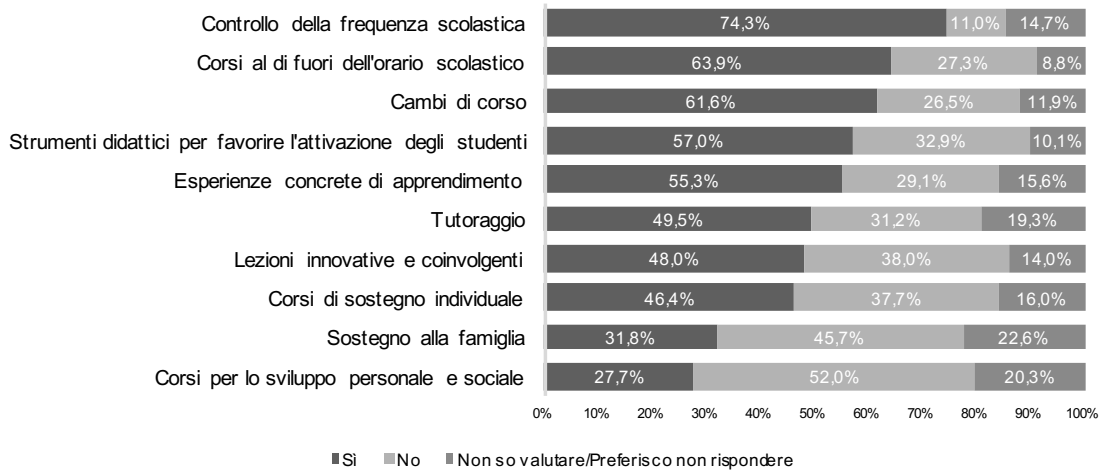

Fig. 22 - Misure di prevenzione e contrasto alla dispersione messe in atto nella propria scuola (studenti)

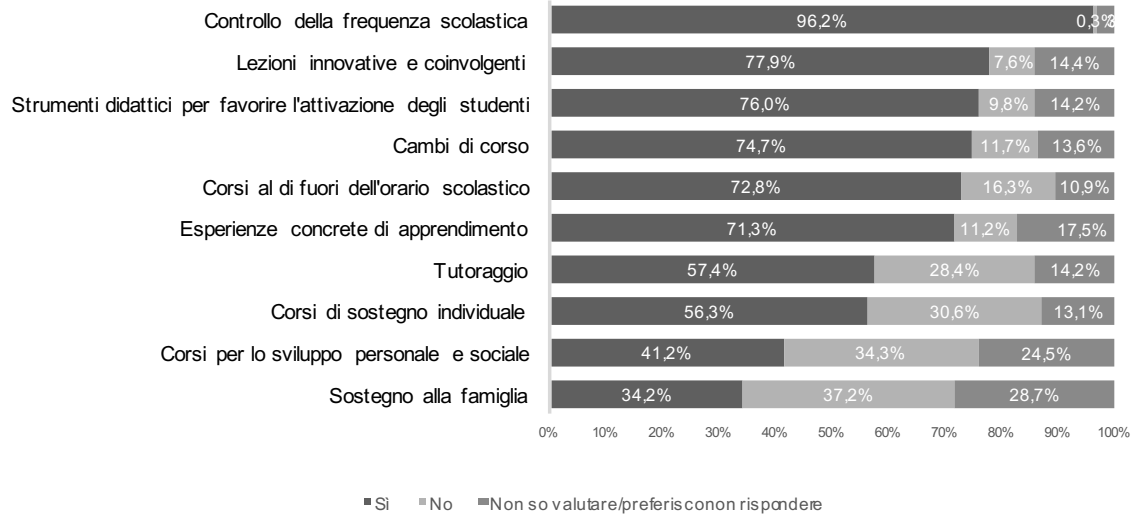

Fig. 23 - Misure di prevenzione e contrasto alla dispersione messe in atto nella propria scuola (docenti)

\subsection{La stima della dispersione scolastica}

L'ultima sezione del questionario è volta alla rilevazione della percezione soggettiva della diffusione del fenomeno della dispersione scolastica.

La stima viene effettuata a tre livelli: da uno più prossimo - quale la scuola di appartenenza (nel caso di studenti e docenti) o la scuola frequentata dal figlio/a (nel caso in cui i rispondenti siano i genitori) - a livelli meno prossimi, ovvero il contesto regionale e quello nazionale.

Va sottolineata qui la differenziazione terminologica utilizzata nello strumento somministrato agli studenti. Mentre, infatti, a docenti e genitori viene 
chiesto di fornire una valutazione in termini percentuali (ad esempio: "Secondo la sua opinione, su 100 studenti della sua REGIONE, quanti sono quelli interessati dal fenomeno della dispersione scolastica (abbandonano o non frequentano o vengono bocciati)?"), per gli studenti si è scelto di richiedere una stima in termini di studenti per classe ("Secondo te, in una classe qualsiasi della tua REGIONE, quanti ragazzi potrebbero essere interessati dal fenomeno della dispersione scolastica (bocciati, a rischio di abbandonare la scuola, frequentano saltuariamente)?").

La scelta, determinata dall'intento di rendere più immediata la comprensione del quesito e favorire la formulazione di una stima più attendibile (lo studente avrebbe più semplicità ad esprimersi rispetto ad un contesto conosciuto, quale è quello della classe), ha rivelato un risvolto negativo per la libertà lasciata agli studenti di indicare qualsiasi valore numerico, persino valori poco plausibili poiché eccedenti la capienza massima di una classe. Si è pertanto deciso di ritenere solo le risposte entro la soglia limite di $10^{7}$. Valori oltre 10 potrebbero essere indice di una scorretta comprensione del quesito, oltre che di una mancata conoscenza del fenomeno, e sono pertanto esclusi dall'analisi.

Le risposte degli studenti sono riassunte in fig. 24 e tab. 2. Dalla distribuzione delle risposte degli studenti (fig. 24) si evidenzia un picco, al livello della scuola di appartenenza, per valori pari a 3: 196 studenti (circa il 23\% delle risposte valide) ritengono che, in una classe qualsiasi della propria scuola, tre soggetti siano generalmente interessati dal fenomeno della dispersione scolastica. Cumulativamente, il $60 \%$ dei rispondenti stima un'incidenza della dispersione inferiore o uguale a tre soggetti per classe (che, in una classe di 25 alunni, rappresentano il 12\%).

A livello regionale, il $37 \%$ delle risposte si concentra tra valori compresi tra 4 e 5 , che corrispondono ad una incidenza della dispersione nelle classi delle scuole umbre tra il $16 \%$ e il $20 \%$.

Infine, a livello nazionale, le risposte presentano due picchi per i valori 5 (incidenza del 20\%) e 10 (incidenza del 40\%). Nell'interpretare questo dato occorre tenere conto di distorsioni derivanti dalla scarsa cognizione del fenomeno e dalla tendenza a rispondere "a caso" rispetto a temi poco conosciuti.

${ }^{7}$ In considerazione del fatto che una classe è composta, mediamente, da 25 alunni, un numero di soggetti interessati dalla dispersione pari a 10 implica un tasso di incidenza della dispersione pari al $40 \%$. Le osservazioni rientranti in questo valore-soglia sono pari a $n=864$. 


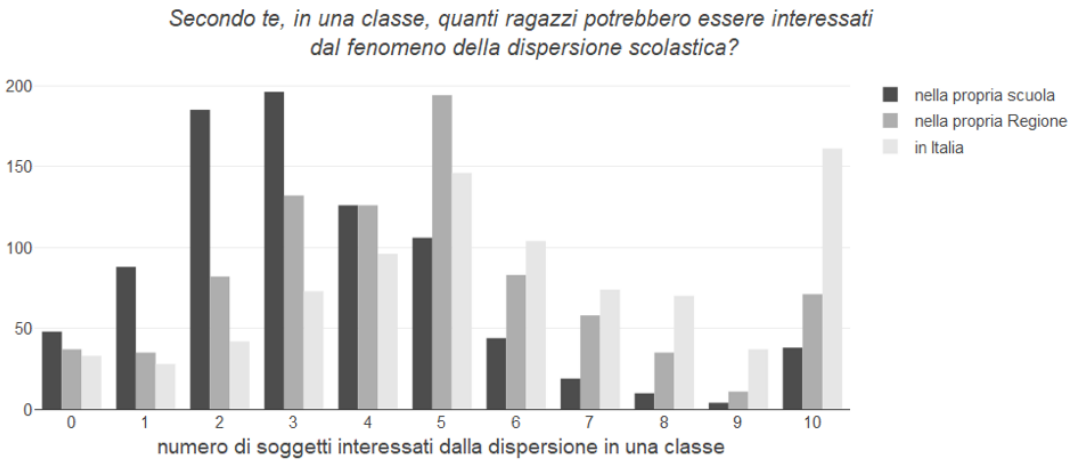

Fig. 24 - Stima della diffusione del fenomeno della dispersione - Distribuzione delle risposte (studenti)

Coerentemente con quanto si nota dalla distribuzione delle risposte, la tab. 2 evidenzia la tendenza a considerare la dispersione scolastica meno diffusa (mediamente 3,4 soggetti per classe) nel contesto più prossimo, ossia la propria scuola, e più consistente a livello regionale (4,7 soggetti in una classe umbra e 5,9 in una classe di una scuola italiana).

Seguono la medesima tendenza le risposte di genitori e docenti. Le figg. 25 e 26 mostrano la distribuzione delle risposte di queste due categorie di rispondenti, mentre la tab. 3 mette a confronto le medie delle stime fornite.

Tab. 2 - Stima della diffusione del fenomeno della dispersione-Media e Percentuale (studenti)

\begin{tabular}{lcc}
\hline \multicolumn{1}{c}{ Livello } & Media & Percentuale $^{*}$ \\
\hline nella propria scuola & 3,4 & $13,60 \%$ \\
nella propria Regione & 4,7 & $18,80 \%$ \\
in Italia & 5,9 & $23,60 \%$ \\
\hline
\end{tabular}

* in rapporto ad una classe media di 25 alunni

Si ricorda che il quesito utilizzato per genitori e docenti chiede di esprimere una valutazione della diffusione del fenomeno della dispersione rapportata a 100 studenti (della propria scuola/della scuola del figlio, di una scuola della propria Regione, di una scuola italiana). 


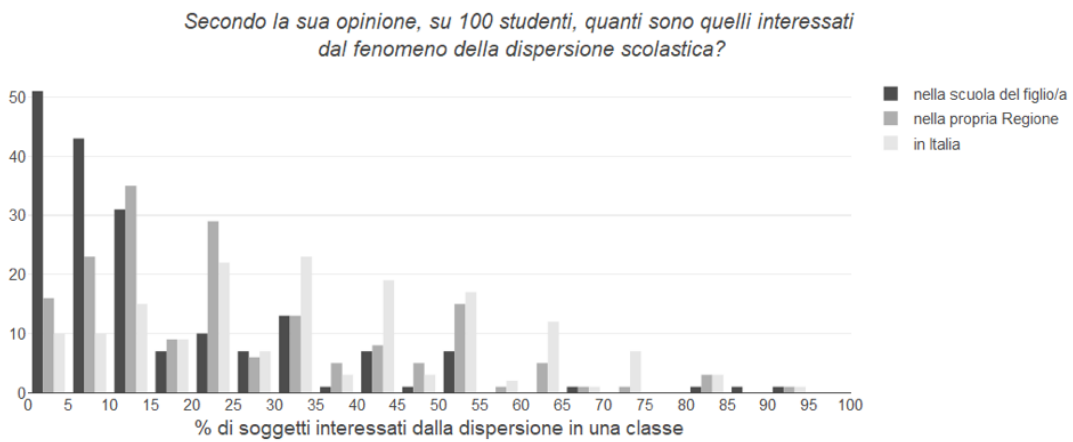

Fig. 25 - Stima della diffusione del fenomeno della dispersione - Distribuzione delle risposte (genitori)

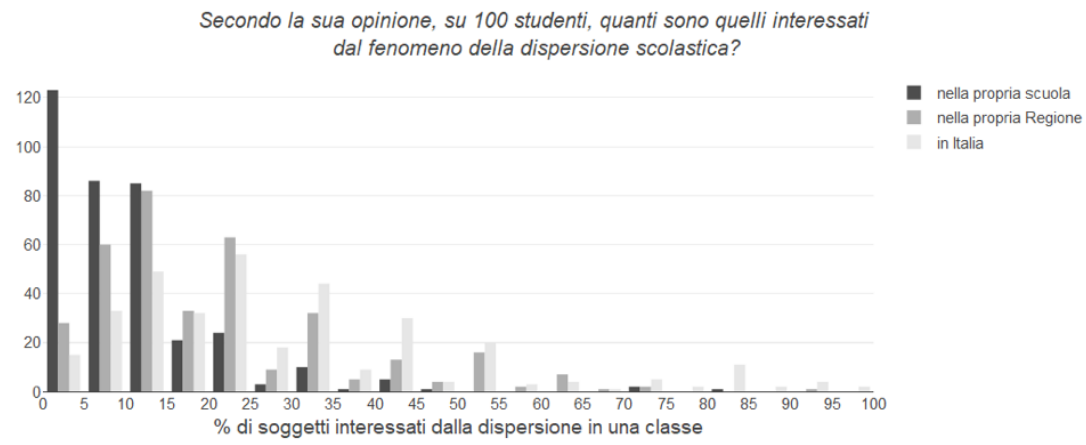

Fig. 26 - Stima della diffusione del fenomeno della dispersione - Distribuzione delle risposte (docenti)

Si evidenziano consistenti differenze nella percezione dell'incidenza della dispersione tra le due tipologie di soggetti: in generale, per i genitori la dispersione scolastica è un fenomeno più diffuso rispetto a quanto ritenuto dagli insegnanti.

La maggior differenza riguarda la valutazione del contesto italiano, con circa 12 punti percentuali di differenza (il $39 \%$ per i genitori e il $27 \%$ per i docenti). La differenza minore (di circa 5 punti percentuali) si situa al livello più prossimo: per i genitori, nella scuola del proprio figlio/a, la dispersione scolastica ha una diffusione del $14,4 \%$; per i docenti, nella scuola in cui si insegna, la diffusione è di circa il $9 \%$. 
Tab. 3 - Stima della diffusione del fenomeno della dispersione - Media delle risposte di genitori e docenti

\begin{tabular}{lcc}
\hline \multicolumn{1}{c}{ Livello } & Media genitori & Media docenti \\
\hline nella scuola & $14,40 \%$ & $8,98 \%$ \\
nella propria Regione & $26,09 \%$ & $18,80 \%$ \\
in Italia & $39,08 \%$ & $27,38 \%$ \\
\hline
\end{tabular}

\subsection{Esperienza di formazione specifica sulla dispersione da parte dei docenti}

All'interno del questionario indirizzato ai docenti è stata inserita un'ulteriore sezione dedicata alla formazione sul tema della dispersione scolastica. Il 65,6\% dei docenti dichiara di non aver mai partecipato a percorsi formativi sul tema. Analizzando la distinzione per livello di scuola, si rileva come siano principalmente i docenti della scuola secondaria di primo grado a non avere alcuna esperienza in merito: solo il 18,4\% risponde "si" contro il 46,7\% dei docenti delle superiori (fig. 27).

\section{Personalmente, ha mai preso parte a percorsi formativi sul tema della dispersione scolastica?}

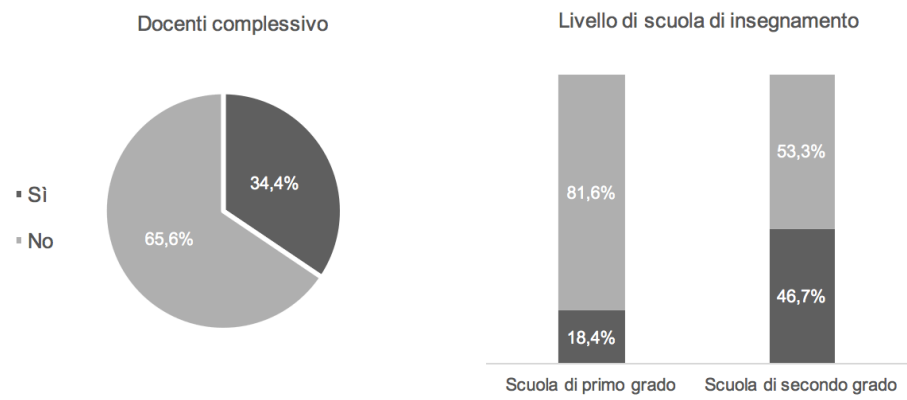

Fig. 27 - Partecipazione a percorsi formativi sul tema della dispersione scolastica

In secondo luogo, è stato chiesto, a chi ha frequentato percorsi formativi sulla dispersione, quanto - su una scala likert a 5 passi (per niente, poco, abbastanza, molto e moltissimo) - ritenga che siano stati utili. L'opinione dei docenti sull'utilità dei corsi frequentati è abbastanza positiva: nel complesso, il 43,3\% li ritiene abbastanza utili, il 26,1\% molto utili e il 7,6\% moltissimo (fig. 28). 
È stato, infine, chiesto ai docenti quanto fossero interessati ad approfondire la formazione sul tema della dispersione. Anche in questo caso la propensione dei docenti ad approfondire l'argomento è tendenzialmente positiva: il $28,8 \%$ si dichiara molto interessato ad approfondire e il $17,3 \% \mathrm{~mol}$ tissimo (fig. 29).

\section{Se ha frequentato percorsi formativi sul tema della dispersione scolastica, quanto} ritiene siano stati utili?
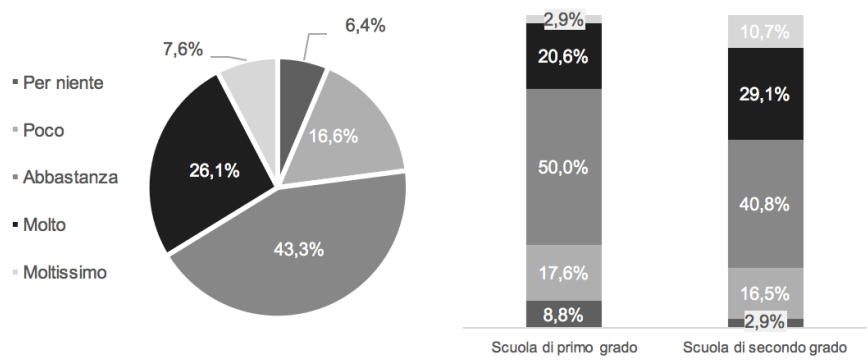

Fig. 28 - Valutazione dell'utilità dei percorsi formativi sulla dispersione scolastica

Quanto è interessato ad approfondire la formazione su questo tema?
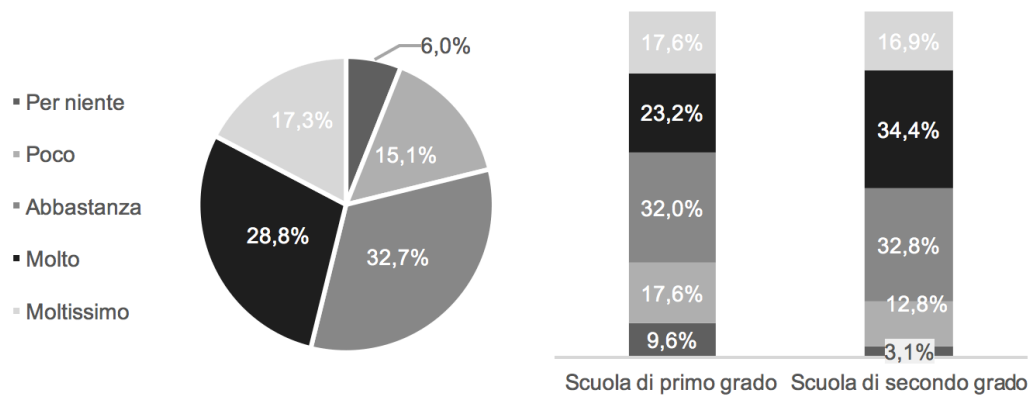

Fig. 29 - Livello di interesse ad approfondire la formazione sul tema della dispersione scolastica

\section{Conclusioni}

Il quadro che emerge dall'indagine condotta è quello di una realtà scolastica umbra, relativamente alla scuola secondaria, vicina al fenomeno della dispersione scolastica e, contemporaneamente, poco (in)formata sul tema. 
La maggior parte degli studenti e dei docenti che hanno risposto al questionario dichiara, infatti, di conoscere soggetti che hanno abbandonato la scuola (oltre il 60\% degli studenti e il 70\% dei docenti). Da questi dati sembra che l'abbandono scolastico non sia un fenomeno raro nel territorio in esame, nonostante le stime nazionali riferiscano tassi di abbandono tra i più bassi.

Di fronte ad una realtà che tocca più o meno direttamente gran parte di studentesse e studenti, questi non sembrano avere gli strumenti per poter leggere correttamente il fenomeno: circa il 76\% dichiara di non aver mai sentito parlare di "dispersione scolastica".

L'evidente mancanza, nelle scuole, di canali informativi sul tema predisposti per gli studenti, si allarga alla formazione specifica dei docenti. Tra quest'ultimi, infatti, si rileva una scarsa conoscenza della dispersione scolastica nei suoi aspetti precipui: ad esempio, solo il $41 \%$ dei docenti intervistati associa fortemente la categoria dei "bocciati" alla dispersione.

Emerge dunque una concezione della dispersione scolastica che rispecchia la confusione terminologica - presente anche nei report e nelle statistiche ufficiali - per cui si tende a far coincidere la dispersione con il solo $a b$ bandono, sottostimando in questo modo un fenomeno più ampio che comprende anche coloro che, sebbene ancora presenti all'interno del sistema di istruzione, non riescono a portare a termine il proprio percorso nei tempi e nei modi più soddisfacenti e funzionali al proprio sviluppo personale e professionale.

La scarsa conoscenza del fenomeno trova parziale spiegazione nel numero di docenti che ha seguito corsi di formazione specifici sul tema, ovvero solo il 35\%. Riguardo a quest'ultimo aspetto, bisogna tuttavia sottolineare la percezione positiva di coloro che hanno partecipato a corsi di formazione in termini di utilità dei corsi stessi e di interesse ad approfondire la propria formazione sul tema: circa 8 insegnanti su 10 forniscono una valutazione positiva in relazione a queste due dimensioni.

Un ulteriore dato interessante, emerso dall'indagine, è la percezione dei fattori di rischio della dispersione. Studenti, docenti e genitori si rivelano, infatti, concordi, nel riconoscere la bassa motivazione allo studio come principale fattore associato alla dispersione scolastica.

Di fronte al riconoscimento dell'importanza della scarsa motivazione come fattore di rischio, si rilevano risposte coerenti, da parte di studenti e genitori, in relazione alla percezione di quali siano le azioni maggiormente efficaci per prevenire la dispersione. Questi, infatti, valutano la didattica attiva come tipologia di azione più efficace. Al contrario, i docenti assegnano un maggiore valore al sostegno individuale e al tutoraggio. 
Quello che pare venir fuori dai risultati appena riassunti è la necessità di un approccio preventivo di tipo ecologico e sistemico, che parta innanzitutto dal predisporre un adeguato piano di (in)formazione sul problema che coinvolga studenti, docenti e genitori.

Tra gli attori implicati nel sistema scolastico, il ruolo ricoperto dai docenti è indubbiamente centrale nell'azione di prevenzione della dispersione, a cui va affiancata una corretta informazione rivolta a studenti e genitori.

Da parte dei docenti conoscere, ad esempio, che la noia e la mancanza di connessione percepita di ciò che si apprende a scuola con il mondo extrascolastico sono tra le motivazioni principali dell'abbandono (Bridgeland, DiIulio e Morison, 2006; Bridgeland et al., 2010), potrebbe consentire loro di riconoscere che le modalità più efficaci per prevenire e contrastare la dispersione non possono situarsi esclusivamente in azioni di sostegno individuale (che, per lo più, finiscono per replicare le stesse modalità didattiche esperite in classe). Analogamente, essere consapevoli che non solo i fattori legati alle pratiche disciplinari, ma anche l'attitudine e il comportamento dei docenti, nonché la qualità del rapporto studente-insegnante, possono influenzare le decisioni degli studenti in ordine ad abbandonare il proprio percorso di istruzione (Pierce 1994; Bennacer, 2000; Potvin e Paradis, 2000; Christenson et al., 2001; Vellos e Vadeboncoeur, 2015; Nairz-Wirth e Feldmann, 2017), così come i loro risultati scolastici (Fredriksen e Rhodes, 2004; Fortin et al., 2006; Englund, Egeland e Collins, 2008), potrebbe spingere i docenti stessi a riflettere sulle proprie modalità di relazione e sulla rilevanza dei propri atteggiamenti nel rafforzare (o indebolire) la motivazione degli alunni. È stato rilevato come molti studenti che decidono di allontanarsi dalla scuola hanno relazioni più povere con gli insegnanti rispetto a coloro che permangono nel sistema scolastico (Glogowski, 2015; Lehr et al., 2003). Stabilire un rapporto interpersonale significativo tra studenti e insegnanti si rileva dunque fondamentale. Altrettando rilevante è puntare sulla promozione della motivazione degli studenti, che, come è noto (cfr., ad esempio, Weber et al., 2013), ha una forte incidenza sul successo formativo.

In ultima analisi, una formazione adeguata dei docenti appare il fulcro di un'azione sistemica di prevenzione e contrasto alla dispersione scolastica che ha come presupposto il riconoscimento dell'importanza di agire su uno dei fattori principali connessi al successo (o all'insuccesso) formativo, ovvero la motivazione di studentesse e studenti - come le visioni riportate nella presente indagine rivelano e la letteratura scientifica conferma. Motivazione all'apprendimento che può essere promossa da una didattica centrata sull'attivazione e il coinvolgimento degli alunni e caratterizzata da una relazione interpersonale docente-studente significativa e, a sua volta, motivante. In 
questa cornice didattica trovano ampia legittimazione le pratiche di lettura ad alta voce e di orientamento narrativo che, come altrove analizzato in questo volume, aiutano a promuovere sia l'empowerment complessivo dell'individuo che specifiche dimensioni cognitive e socio-relazionali, indispensabili per supportare il successo formativo e un clima relazionale positivo nel contesto classe.

\section{Riferimenti bibliografici}

Alivernini F., Manganelli S. e Lucidi, F. (2017), "Dalla povertà educativa alla valutazione del successo scolastico: concetti, indicatori e strumenti validati a livello nazionale", Journal of Educational, Cultural and Psychological Studies (ECPS Journal), 1, 15: 21-52.

Bartolucci M. e Batini F. (2016), Ascoltare studenti, insegnanti, dirigenti: che cos'è la dispersione? in Batini F. e Bartolucci M., a cura di, Dispersione scolastica. Ascoltare $i$ protagonisti per comprenderla e prevenirla, pp. 123-133, FrancoAngeli, Milano.

Batini F. (2014), Drop-out, Fuori|onda, Arezzo.

Bennacer H. (2000), "How the Socioecological Characteristics of the Classroom Affect Academic Achievement”, European Journal of Psychology of Education, 15, 2: 173-189.

Bridgeland J.M., Balfanz R., Moore L.A. and Friant R.S. (2010), "Raising Their Voices: Engaging Students, Teachers, and Parents to Help End the High School Dropout Epidemic", Civic Enterprises.

Bridgeland J.M., DiIulio Jr J.J. and Morison K.B. (2006), “The silent epidemic: Perspectives of high school dropouts", Civic Enterprises.

Bunting M. and Moshuus G. (2017), "Young peoples' own stories about dropping out in Norway: An indirect qualitative approach", Acta Didactica Norge, 11, 2: 20-sider.

Christenson S.L., Sinclair M.F., Lehr C.A. and Godber Y. (2001), "Promoting successful school completion: Critical conceptual and methodological guidelines", School Psychology Quarterly, 16, 4: 468.

Davis K.S. and Dupper D.R. (2004), "Student-teacher relationships: An overlooked factor in school dropout", Journal of human behavior in the social environment, 9, 1-2: 179-193.

Dynarski M. and Gleason P. (2002), "How can we help? What we have learned from recent federal dropout prevention evaluations", Journal of Education for Students Students Placed At Risk, 7, 1: 43-69.

Doll J.J., Eslami Z. and Walters L. (2013), "Understanding why students drop out of high school, according to their own reports: Are they pushed or pulled, or do they fall out? A comparative analysis of seven nationally representative studies", Sage Open, 3, 4.

Englund M.M., Egeland B. and Collins W.A. (2008), "Exceptions to High School Dropout Predictions in a Low-Income Sample: Do Adults make a Difference?”, Journal of Social Issues, 64, 1: 77-94.

Eurostat (2017), Early leavers from education and training, testo disponibile al sito: http://ec.europa.eu/eurostat/statistics-explained/index.php/Early_leavers_from_education_and_training.

Fredriksen K. and Rhodes J. (2004), "The role of Teacher relationships in the Lives of Students", New Directions for Youth Development, 103: 45-54.

Fortin L., Marcotte D., Potvin P., Royer E. and Joly J. (2006), “Typology of Students at risk of Dropping out of School: Description by Personal, Family and School Factors", European Journal of Psychology of Education, 21, 4: 363-383. 
Gallagher C.J. (2002), "Stories from the strays: What dropouts can teach us about school”, American Secondary Education, 36-60.

Glogowski K. (2015), "What works in dropout prevention: Research evidence, pathways to education design, and practitioner knowledge", Pathways to Education Canada, Toronto, $\mathrm{ON}$.

Jeffries R.B., Nix M. and Singer C. (2002), "Urban American Indians "dropping" out of traditional high schools: Barriers \& bridges to success", The High School Journal, 85, 3: 3846.

Jimerson S., Egeland B., Sroufe L.A. and Carlson B. (2000), "A prospective longitudinal study of high school dropouts examining multiple predictors across development", Journal of school psychology, 38, 6: 525-549.

Knesting-Lund K., Reese D. and Boody R. (2013), “Teachers' perceptions of high school dropout and their role in dropout prevention: An initial investigation", Journal of Studies in Education, 3, 4: 57-71.

Lehr C.A., Hansen A., Sinclair M.F. and Christenson S.L. (2003), "Moving beyond dropout towards school completion: An integrative review of data-based interventions", School Psychology Review, 32, 3: 342-365.

Miur (2017), La dispersione scolastica nell'a.s. 2015/2016 e nel passaggio all'a.s. 2016/2017, testo disponibile al sito:

http://www.miur.gov.it/documents/20182/0/Focus+La+dispersione+scolastica/9bc1c11b-1c40-4029-87ba-40b8ba2bc2bf?version=1.1.

Nairz-Wirth E. and Feldmann K. (2017), “Teachers' views on the impact of teacher-student relationships on school dropout: a Bourdieusian analysis of misrecognition", Pedagogy, Culture \& Society, 25, 1: 121-136.

Obasohan A.N. and Kortering L. (1999), "Dropping Out of School: Comparing the Perceptions of Teachers and Dropouts", Journal of At-Risk Issues, 5, 2: 19-26.

Pierce C. (1994), "Importance of Classroom Climate for at-risk Learners", The Journal of Educational Research, 88, 1: 37-42.

Potvin P. and Paradis L. (2000), "Facteurs de réussite dès le début de l'éducation préscolaire et primaire, Études et recherches, 5 (3)". Québec, Qc: Rapport de recherche présenté au Centre de recherche et d'intervention sur la réussite éducative et scolaire.

Rosenthal R. (1973), "The Pygmalion Effect Lives", Psychology today.

Tyler J.H. and Lofstrom M. 2009, "Finishing High School: Alternative Pathways and Dropout Recovery", America's High Schools, 19, 1: 77-103.

Vellos R.E. and Vadeboncoeur J.A. (2015), "Rebuilding Attendance Practices with Youth: The role of Social mediation", Educational Studies, 41, 1-2: 91-108.

Weber HS., Lu L., Shi J. and Spinath F.M. (2013), "The roles of cognitive and motivational predictors in explaining school achievement in elementary school", Learning and Individual Differences, 25: 85-92. 


\title{
L'atteggiamento dei docenti nei confronti della dispersione scolastica
}

\author{
di Cecilia Bini, Leonardo Fasi, Elisa Morini e Alessandra Sisto
}

\section{Introduzione}

La dispersione scolastica è ancora oggi un fenomeno numericamente consistente e con gravi conseguenze in termini di costi individuali, sociali e comunitari (Save the Children, 2017).

Alla luce della rilevanza del fenomeno, all'interno del corso di Metodologia della ricerca, dell'osservazione e della valutazione (A.A. 2017-2018) si è scelto di condurre un'indagine sulla percezione della dispersione da parte degli studenti delle scuole secondarie di primo e secondo grado del territorio umbro e dei rispettivi insegnanti e genitori.

I temi affrontati e la successiva ricerca sul campo, che ha coinvolto tutti gli studenti del corso, hanno contribuito a sensibilizzarci particolarmente rispetto a questo argomento, spingendoci ad approfondire il tema in diverse direzioni. In particolare, abbiamo voluto, in primo luogo, esaminare il punto di vista e il vissuto dei drop-out per poi affrontare, con maggior consapevolezza, l'analisi dei dati sull'atteggiamento dei docenti.

\section{Il punto di vista dei Drop-Out}

I "drop-out" sono, secondo un termine di origine anglosassone, i giovani fuoriusciti da un percorso d'istruzione, formativo o lavorativo, senza aver acquisito una certificazione formale. Si tratta di moltissimi ragazzi e ragazze con percorsi, motivazioni, trascorsi molto diversi, troppo spesso rappresentati in modo semplificativo come aventi le medesime caratteristiche e definiti attraverso le loro mancanze.

Molti dei soggetti "drop-out" non hanno abbandonato la scuola perché non avevano voglia di studiare, perché non ce la facevano a stare dietro allo 
studio, o per altri "deficit" del singolo, ma piuttosto a causa di un "gap" situato all'interno del sistema scolastico che non è stato in grado di coinvolgerli e di comprenderli:

Io, sinceramente, non ho una visione molto positiva della scuola...non mi sembra molto utile... sì, impari certe cose ma impareresti di più facendo esperienze pratiche di vita. Il momento che preferisco durante la lezione è il confronto sui vari argomenti e i dibattiti... (Corso, 17 anni) (Batini, 2014, p. 52).

Io ho deciso di abbandonare la scuola principalmente a causa della matematica e del professore stesso... c'è stato un sacco di casino... mi offendeva perché sono robusta... lui puntava parecchio su questa cosa e alla fine mio padre si arrabbiò. Così ho scoperto che il problema non ero io ma il professore di matematica che mi aveva convinta che non avessi le capacità. (Michela,17 anni) (ivi, p. 57).

«Le parole di questi ragazzi non possono», afferma Burgio, «allora non interrogare la scuola e gli insegnanti, farci pensare al fatto che questi ragazzi non si sono sganciati dalla scuola ma sono stati abbandonati da questa scuola che non ha saputo includerli, motivarli, comprenderli, che forse non ha saputo ascoltare il loro implicito racconto quando frequentavano le nostre aule, silenziosa o fracassona che fosse la loro presenza, timida o aggressiva, egocentrica o auto-escludentesi» (2014, p. 177). «Se i dropout sono lo specchio delle contraddizioni del sistema di istruzione, sono al tempo stesso la testimonianza e la traiettoria di possibili percorsi alternativi» (Benvenuto, 2014, p. 9). Molte altre testimonianze mostrano, infatti, come i ragazzi desiderino che si sviluppi un buon percorso formativo:

Vorrei che una volta entrati in classe i professori ci dicessero che sarà un anno impegnativo, ma divertente e che ci aiuteranno quando non capiamo o che saranno sempre nel positivo quando spiegano, e che avranno quella passione che contagerà tutta la classe in modo che ci piacerà tutto (Classe prima, ITIS) (De Carlo e Evangelista, 2016, p. 91).

Mi piacerebbe sentirmi dire che non è così importante avere il 10 a tutte le materia, ma sarebbe molto importante uscire dalla scuola con la capacità di seguire un telegiornale e capirlo. Mi piacerebbe che ci dicessero che le emozioni, le emozioni sono una delle cose più importanti nella vita. Che i sogni non sono mai sbagliati che non siamo mai troppo grandi. Che non esistono sogni irrealizzabili, ma solo persone che smettono di crederci. Vorrei che mi facessero capire bene l'importanza di credere nei miei sogni e in me stessa (Classe prima, ITIS) (ivi, p. 91). 
La scuola dovrebbe chiedersi quale ruolo può avere e cosa concretamente può fare per prevenire e contrastare il fenomeno della dispersione scolastica. Per questo è fondamentale valutare, da un lato, quanto di questo fenomeno gli studenti conoscono e, in secondo luogo, approfondire il punto di vista dei docenti.

\section{La percezione degli studenti}

Prima di approfondire il punto di vista dei docenti verso la dispersione, è opportuno sottolineare il livello di conoscenza del fenomeno da parte degli studenti, vera cartina di tornasole per una scuola funzionale (Bartolucci e Batini, 2016).

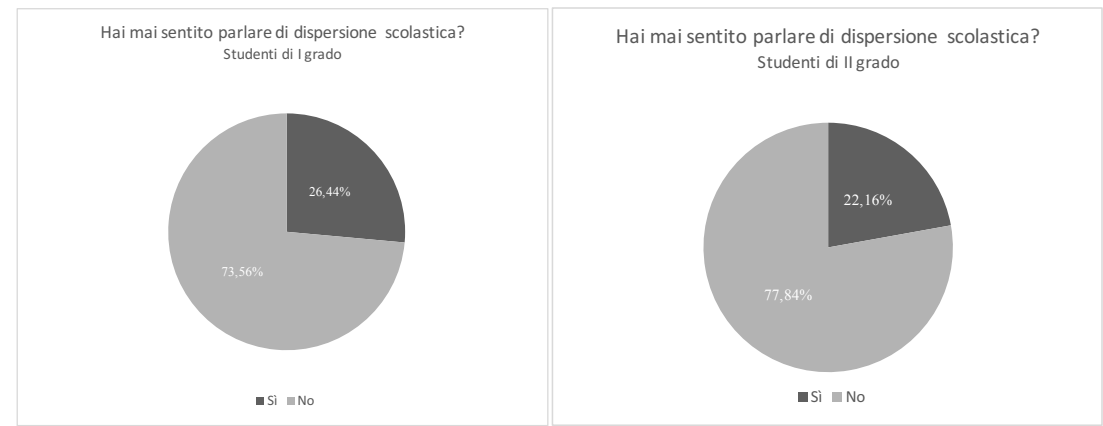

Fig. 1 - Conoscenza del fenomeno della dispersione scolastica da parte degli studenti

La figura 1 mostra come la grande maggioranza degli studenti, in maniera congruente tra $\mathrm{i}$ due gradi scolastici, non ha mai sentito parlare di dispersione: hanno infatti risposto "No" il 73,56\% degli studenti delle scuole secondarie di primo grado e il $77,84 \%$ degli studenti delle scuole secondarie di secondo grado.

Focalizzandoci sulle scuole secondarie di secondo grado, è interessante notare come non vi sia differenza neanche tra le varie tipologie di scuole (liceo; istituto tecnico; istituto professionale) (fig. 2). 


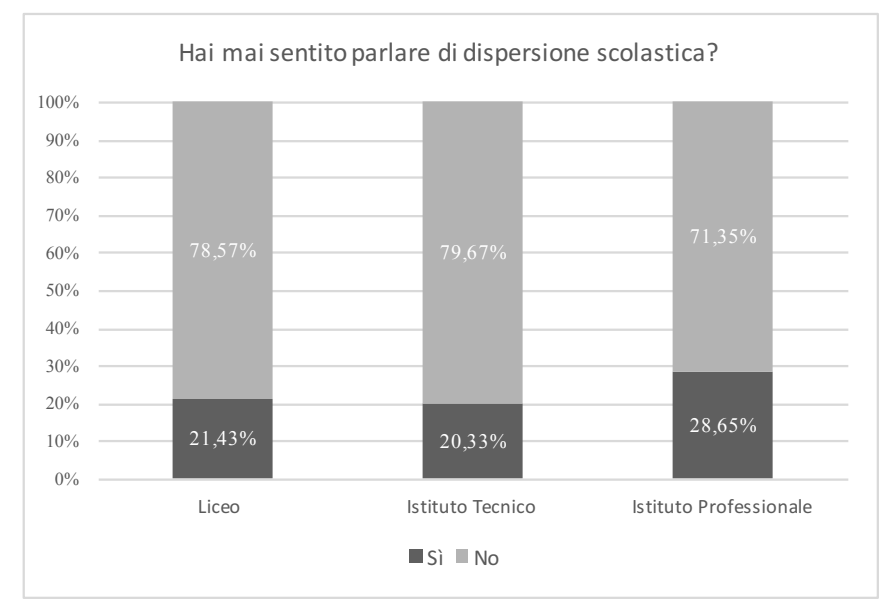

Fig. 2 - Conoscenza della dispersione scolastica da parte degli studenti per tipologia di scuola

Dai dati appena evidenziati, la conoscenza del fenomeno da parte degli studenti risulta quindi essere scarsa, nonostante la dispersione scolastica sia molto diffusa in Italia. Prendendo in considerazione i dati ufficiali pubblicati dal MIUR (2017), l'indice della dispersione, sebbene mostri un miglioramento nel corso degli ultimi anni, si attesta per l'anno 2016 al 13,8\%, ancora al di sotto del target del 10\% stabilito dalla Strategia Europa 2020.

Il luogo deputato per informare i ragazzi sul tema, contribuendo così alla prevenzione del problema, è senza dubbio la scuola. È spontaneo a questo punto porsi alcune domande chiave: La scuola tratta questo argomento? $\dot{E}$ realmente interessata a farlo? Pianifica ed attua di conseguenza progetti di sensibilizzazione? Riceve adeguate informazioni in merito?

Cercheremo di dare risposta a tali quesiti nei paragrafi successivi, andando ad indagare l'atteggiamento del corpo docenti.

\section{La formazione dei docenti}

\subsection{Frequenza ai corsi di formazione sul tema della di- spersione}

Andiamo quindi ad osservare quale sia l'atteggiamento dei docenti, partendo dalla rilevazione di quanti hanno frequentato percorsi formativi sul tema della dispersione scolastica. 
La fig. 3 mostra la partecipazione a percorsi formativi sul tema, per anzianità di servizio.

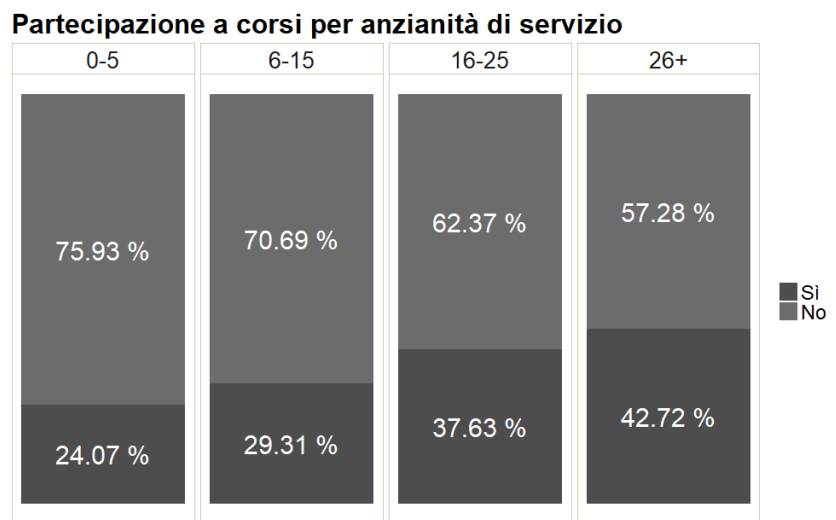

Fig. 3 - Frequenza di percorsi formativi sul tema della dispersione scolastica per anzianità di servizio

Notiamo che minore è il grado di anzianità di servizio, che corrisponde al $15 \%$ del totale, e minore è la frequenza dei percorsi formativi. Considerando invece le due fasce intermedie di anzianità di servizio, cioè quelle che vanno dai sei ai venticinque anni, soltanto un 1 docente su 3 si è formato $(33,47 \%)$.

La fig. 4 rappresenta le risposte alla stessa domanda, con focus sui gradi scolastici.

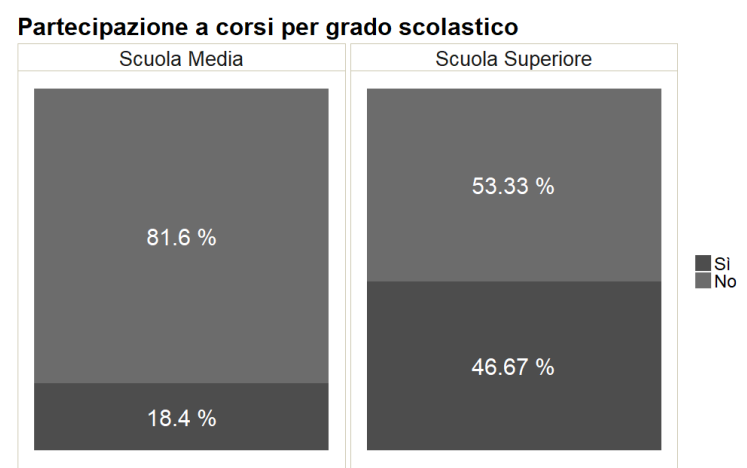

Fig. 4 - Frequenza di percorsi formativi sul tema della dispersione scolastica per livello di scuola 
Emerge qui una prima discrepanza tra i due gradi scolastici: nelle scuole secondarie di primo grado meno di 1 docente su 5 ha preso parte a percorsi formativi $(18,4 \%)$, mentre nelle scuole secondarie di secondo grado poco meno della metà si è formata $(46,67 \%)$.

\subsection{Atteggiamento di coloro che hanno già partecipato a percorsi formativi}

Dal punto di vista di chi ha già intrapreso un percorso di formazione a riguardo, si può constatare che circa il $57 \%$ (ottenuto sommando le due risposte "molto" 23,02\% e "moltissimo" 34,13\%) è ancora molto interessato ad approfondire il tema (fig. 5).

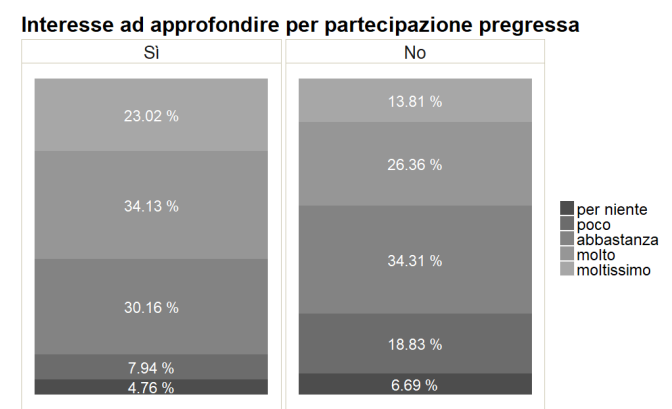

Fig. 5 - Interesse ad approfondire la formazione sul tema della dispersione scolastica in relazione alla partecipazione pregressa a percorsi formativi sullo stesso tema

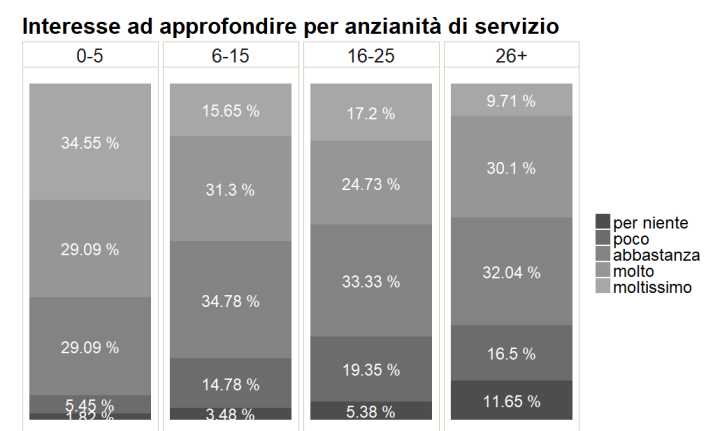

Fig. 6 - Interesse ad approfondire la formazione sul tema della dispersione scolastica in relazione all'anzianità di servizio 
Dalla fig. 6 si evince che i docenti con minor anzianità di servizio sono nettamente più interessati ad approfondirne la formazione, in quanto se prendiamo in esame i gradi "molto" e "moltissimo", si raggiunge circa il 65\% da parte dei docenti con meno di 5 anni di insegnamento.

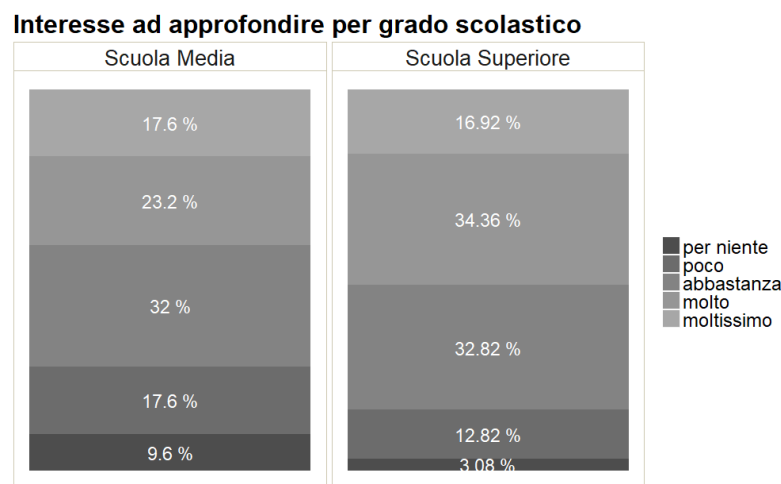

Fig. 7 - Interesse ad approfondire la formazione sul tema della dispersione scolastica in relazione al livello di scuola

Infine, dall'analisi per grado scolastico, è possibile notare un interesse lievemente maggiore tra i docenti delle scuole di primo grado rispetto a quelli delle scuole di secondo grado (circa il 10\% in più) (fig. 7).

In secondo luogo è stato chiesto a quei docenti che hanno preso parte a percorsi formativi sulla dispersione scolastica quanto ritenessero utili tali approfondimenti.

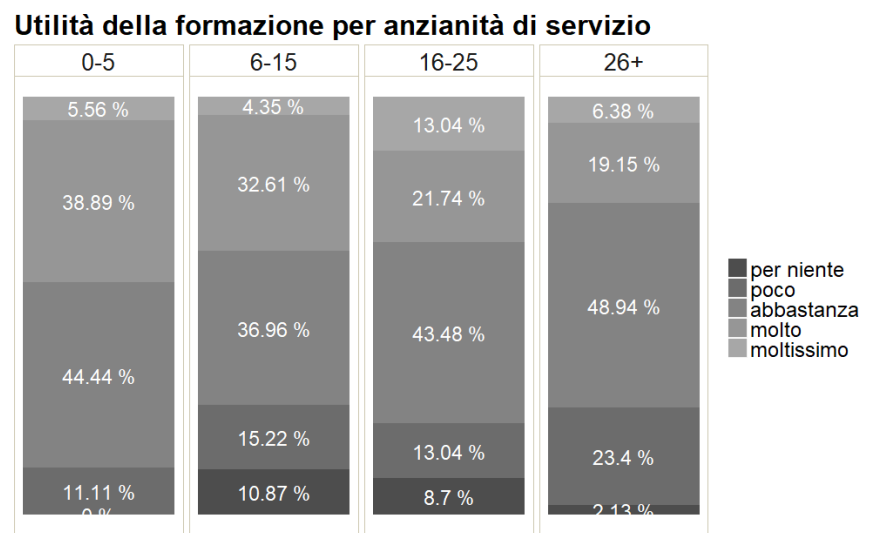

Fig. 8 - Valutazione dell'utilità dei percorsi formativi sulla dispersione scolastica in relazione all'anzianità di servizio 
Si evince che, al crescere dell'anzianità di servizio, si ha una minor considerazione sull'utilità (fig. 8); infatti, coloro che hanno meno esperienza di servizio (fascia $0-5$ anni) ritengono più utili i corsi $(44,45 \%$ sommando $i$ gradi "molto" e "moltissimo"), mentre la maggioranza si colloca in un atteggiamento intermedio, tendente al positivo. Focalizzando l'attenzione sulla fascia centrale di anzianità (da 6 ai 25 anni), che corrisponde al 56,95\% del nostro campione dei docenti, emerge un $9,5 \%$ di completa inutilità.

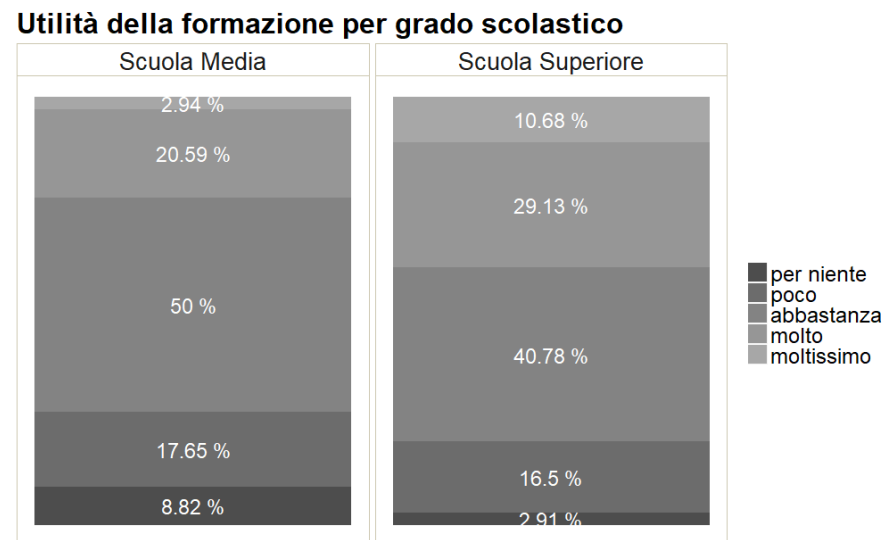

Fig. 9 - Valutazione dell'utilità dei percorsi formativi sulla dispersione scolastica in relazione al livello di scuola

Inoltre i docenti delle scuole secondarie di secondo grado ritengono $\mathrm{i}$ corsi formativi di maggior utilità per un $17 \%$ in più rispetto alle secondarie di primo grado (fig. 9).

\section{Le risposte delle scuole e la loro collaborazione al progetto di ricerca}

A livello processuale, la prima evidenza che abbiamo raccolto è stata l'elevato numero di rifiuti da parte delle scuole nel collaborare al progetto di ricerca: la metà delle scuole contattate, infatti, non ha collaborato. Nel dettaglio: su 61 scuole contattate, 30 hanno rifiutato di collaborare, di cui 21 scuole secondarie di primo grado e 9 scuole secondarie di secondo grado; mentre delle 31 scuole che hanno aderito, 12 erano di primo grado e 19 di secondo grado. Analizzando le risposte delle scuole per gradi scolastici, nelle secondarie di primo grado ci sono stati 21 rifiuti contro 12 adesioni. A nostro 
parere questo dato potrebbe essere indice di una scarsa volontà di collaborazione con l'Università.

Riteniamo infine importante segnalare che in una scuola, ben due famiglie hanno ritirato i propri figli nel giorno della somministrazione del questionario, supponiamo in ragione dello stesso.

\section{Considerazioni conclusive}

\subsection{Criticità emerse}

Rispetto agli interrogativi che ci eravamo posti inizialmente, ovvero se la scuola tratti o meno l'argomento, se sia davvero interessata a farlo, se pianifichi e metta in pratica progetti di sensibilizzazione, e se riceva adeguate informazioni in merito, l'analisi dei dati da noi qui riportata, circa l'atteggiamento dei docenti, ha fatto emergere una serie di risposte negative.

Inoltre, l'elevato numero di scuole che hanno rifiutato la collaborazione con l'Università, può essere inserito in questo quadro generale di disinteresse verso questa tematica così delicata e determinante.

Infine, in base alla nostra esperienza diretta nella somministrazione dei questionari, abbiamo riscontrato una trascuratezza e un disimpegno generale da parte dei docenti stessi; molte domande, infatti, sono state lasciate vuote, e spesso si è verificato un effetto seriale delle risposte "non so valutare/preferisco non rispondere".

I pochi feedback che abbiamo avuto modo di ricevere personalmente, durante o a seguito della somministrazione, sono stati: la noia, l'eccessiva lunghezza dei questionari e l'impegno richiesto nel compilarli. Questo ha contribuito a farci ipotizzare lo scarso interesse verso la questione. La scuola, di fatto, sembra non attuare nessun progetto di sensibilizzazione a riguardo, né tanto meno sembrerebbe ricevere adeguate informazioni in merito.

A nostro parere, riuscire a tessere una relazione positiva tra scuole e Università, in termini di cooperazione e di progettualità verso un fine comune (la lotta alla dispersione scolastica) potrebbe essere una delle possibili vie da intraprendere per contrastare il fenomeno. 


\subsection{Prospettive future e buone pratiche}

Al fine di fornire ai docenti gli strumenti, conoscitivi e metodologici, per poter svolgere un efficace ruolo di prevenzione e contrasto alla dispersione, potrebbe essere utile prevedere corsi di formazione sul tema (eventualmente rendendoli obbligatori), sia presso le scuole polo, tramite una pianificazione idonea ad opera dell'USR Umbria (nell'attuale piano di formazione 2016$2019^{1}$ non si parla di "dispersione"), sia tramite agenzie formative qualificate, che consentano ai docenti l'accesso ai corsi grazie alla "carta del Docente".

In termini concreti, per un'informazione più diffusa ed accessibile, riepiloghiamo nella tabella 1 alcune metodologie didattiche che possono coinvolgere maggiormente gli studenti rispetto alla lezione frontale, promuovendo così un apprendimento significativo.

Tab. 1 - Esempi di metodologie didattiche partecipative (Bartolucci e Batini, 2016)

\begin{tabular}{|c|c|}
\hline Lezione-ricerca & Learning by doing \\
\hline Lezione costruttivista & Project work \\
\hline Lezione visiva & Role playing \\
\hline Lezione mista & Outdoor training \\
\hline Lezione dialogata & Brainstorming \\
\hline Flipped Lesson & Problem solving \\
\hline Lezione laboratorio & E-learning \\
\hline Lezione-esercitazione & Cooperative learning \\
\hline Lezione-gioco & Pear education \\
\hline Lezione/restituzione & $\begin{array}{c}\text { attività-laboratorio (musica, scrittura, } \\
\text { apprendimento cooperativo, } \\
\text { creazione di documentari, } \\
\end{array}$ \\
\hline
\end{tabular}

Quanto suggerito può prendere forma nelle parole e nei desideri di tutti quei ragazzi che si sono dis-persi, chi per demotivazione, chi per frustrazione, chi per incomprensione o a causa di scoraggiamento:

A volte penso che se la scuola fosse stata diversa io non mi sarei persa [...]. Io vorrei imparare a conoscere la vita, soprattutto la mia. Voglio la dimostrazione che le cose trattate abbiano a che fare realmente con essa. [...] in realtà i banchi, a scuola, me li sogno diversi, belli rotondi, dove nessuno è più importante di altri e i docenti possono sedertisi accanto e rapportarsi uno ad uno, senza starsene sulla loro cattedra.

\footnotetext{
http://istruzione.umbria.it/news2017/formazione/pub1/03_Sintesi_Piano_Nazionale_Formazione_Docenti.pdf.
} 
Un'aula diversa dal solito, senza le mura perché 1'aula più bella dovrebbe essere il mondo stesso. [I professori] dovrebbero capire che la mia noia dipende da loro, perché dovrei credere io per prima ad una cosa a cui non credono neanche loro?! [...] Prof. ...che forse mi stai ascoltando, vorrei dirti che io sono la tua più grande sfida, io ragazza che rimango indietro con lo studio e non mi applico... ma che ho tanta voglia di fare le cose che mi piacciono... io, proprio io, dovrei essere il tuo più grande tormento. [...] Se solo tu mi potessi convincere che la scuola possa essere davvero utile... beh allora forse io tornerei a scuola ${ }^{2}$.

\section{Riferimenti bibliografici}

Bartolucci M. e Batini F. (2016), C'era una volta un pezzo di legno. Un progetto Student Voice per le scuole a zero dispersione della Rete di Gubbio, FrancoAngeli, Milano.

Batini F. e Bartolucci M., a cura di (2016), Dispersione Scolastica, FrancoAngeli, Milano.

Batini F. (2014), Drop-out, Fuorionda, Arezzo.

Benvenuto G. (2014), Di cosa parliamo quando parliamo di drop-out?, in Batini F., Dropout, pp. 1-9, Fuorionda, Arezzo.

Burgio G. (2014), Postfazione, in Batini F., Drop-out, pp. 175-177, Fuorionda, Arezzo.

De Carlo M.E. e Evangelista M. (2016), Il progetto Orientadropout-leggere, orientare, raccontare la scuola per uscire dalla dispersione, in Batini F. e Bartolucci M., a cura di, Dispersione scolastica. Ascoltare i protagonisti per comprenderla e prevenirla, pp. 6797, FrancoAngeli, Milano.

Miur (2017), La dispersione scolastica nell'a.s. 2015/2016 e nel passaggio all'a.s. 2016/2017, testo disponibile al sito:

http://www.miur.gov.it/documents/20182/0/Focus+La+dispersione+scolastica/9bc1c11b-1c40-4029-87ba-40b8ba2bc2bf?version=1.1.

Save the Children (2017), Futuro in partenza? L'impatto delle povertà educative in Italia, testo disponibile al sito:

https://www.savethechildren.it/cosa-facciamo/pubblicazioni/futuro-partenza.

Siti web consultati:

http://www.almatu.it/images/pdf/Milanese_Tiziana.pdf

http://www.sipi-adler.it/wp-content/uploads/2015/04/067_CD_Rivoiro.pdf

$\mathrm{http}: / / \mathrm{www}$.qualepsicologia.com/wp-content/uploads/2016/11/41-51-La-dispersione-scolastica-copia.pdf

https://books.google.com/books/about/Marginalia.html?hl=it\&id=KX3huIYlj9QC

http://www.laricerca.loescher.it/istruzione/593-drop-out-storia-di-un-rovesciamento.html

https://books.google.com/books/about/Nel_conflitto_delle_emozioni_Prospet-

tive.html?hl=it\&id=Ue-7BgEmphsC

${ }^{2}$ Estratto di testo scritto da Elisa Morini, frutto di documentazione ed empatizzazione personale. 


\title{
La dispersione universitaria e il processo di inclusione degli studenti con disabilità e/o con DSA
}

\author{
di Laura Arcangeli e Moira Sannipoli
}

\section{Una definizione complessa}

Negli ultimi anni sono cresciuti in maniera significativa gli studi nazionali ed internazionali dedicati al tema della dispersione in ambito universitario. La complessità che caratterizza comunque il fenomeno evidenzia ancora la necessità di costruire una dimensione semantica condivisa e al tempo stesso una lettura più trasversale ed ecologica possibile che provi a superare alcune derive interpretative rispetto al fenomeno stesso (Cfr. Colombo, 2010; Batini e Bartolucci, 2016).

L'esigenza epistemologica di andare a declinare l'intreccio di fattori che ne facilitano l'insorgenza diventa essenziale anche per poter definire misure di intervento adeguate ed efficaci e che chiamino dinamicamente in causa, nella loro molteplicità, tutti gli elementi del sistema: una sorta di accomodamento ragionevole tra scuola secondaria, studente, università (Cfr. Di Pietro e Cutillo, 2008; Heublein et al.; 2003; 2010; Benvenuto e Carci, 2010).

Nel panorama internazionale abbandono, interruzione e/o deviazione del percorso negli studi universitari è spesso descritto con termini quali underachievement, departure, failure, non-continuance, non-completion: in questi contesti il fenomeno è letto in una dimensione per lo più individuale che si contrappone ad un modo funzionale e di profitto di stare nel percorso universitario. L'eziologia del fenomeno è spesso legata allo studente stesso, richiamando sue componenti cognitive, emotive, relazionali e motivazionali, che sembrano non conformarsi in maniera adeguata al sistema accademico (Cfr. McCall, 1994; Balduf, 2009).

Altre interpretazioni si rifanno, invece, ad una matrice ermeneutica che chiama maggiormente in causa il contesto istituzionale universitario, non 
sempre ambiente in grado di facilitare l'apprendimento e di fatto responsabile di un corto circuito tra lo studente stesso e l'istituzione universitaria chiamata ad esercitare una capacità di "retention", cioè di mantenimento e di accompagnamento dello studente stesso in situazione di "attrition", di non avanzamento (Berger e Lyons, 2005; Garza e Bowden; 2014).

Le analisi del fenomeno dispersione richiede quindi un approccio complesso che prediliga una logica connettiva piuttosto che esclusiva, che cerchi di mettere insieme, in maniera dialogica e interlocutoria, attori e azioni che richiamano un piano politico, culturale e di pratiche, dove non c'è sbilanciamento tra una centratura sullo studente o sull'ambiente universitario e sociale.

Questa modalità di lettura è strettamente influenzata da alcuni interventi che nel dibattito internazionale hanno di fatto messo l'accento sull'importanza di definire degli obiettivi comuni per tutti i Paesi membri, considerati essenziali per una società della conoscenza.

La Strategia di Lisbona (2000) con la declinazione dei "cinque livelli di riferimento (benchmark) del rendimento medio europeo" aveva già implicitamente messo l'accento sul tema del contrasto agli abbandoni, obiettivo dichiarato specificatamente sia nel "Processo di Bologna" che nel D. M. $509 / 1999$ che ha riformato gli studi universitari in Italia, proponendo la cosiddetta formula del " $3+2$ ".

La Strategia Europa 2020 (2010) con il riconoscimento della necessità della promozione di una crescita intelligente, sostenibile e inclusiva ha riproposto un'attenzione sul tema, individuando una serie di obiettivi precisi da raggiungere appunto entro il 2020, quali la diminuzione del tasso di abbandono scolastico a meno del $10 \%$ e l'aumento del tasso dei giovani laureati sopra il $40 \%$.

Nonostante ciò a livello europeo non esiste un indicatore ad hoc per il monitoraggio delle carriere accademiche. Nel 2009 l'OCSE aveva proposto come indicatore il tasso di completamento (completion rate), che fa riferimento agli studenti che hanno completato il loro percorso di studi in un arco di tempo che va dalla loro immatricolazione al numero corrispondente alla durata legale del corso. In questa indagine erano stati utilizzati due metodi: il true cohort method, una sorte di coorte di immatricolati seguita nel tempo, misurando il numero di studenti che avevano conseguito il titolo in 8-10 anni e il cross-section cohort method, mettendo in relazione tutti i laureati di un anno agli immatricolati di un certo numero di anni prima, corrispondente alla durata legale del corso. In Italia, in assenza allora di una anagrafe degli studenti, il fenomeno fu analizzato con questo secondo criterio, collocandola all'ultimo posto tra tutti i paesi europei, con una distanza significativa agli 
altri Paesi (45,3 contro 64 per la Francia, 73,9 per la Spagna, 76,9 per la Germania, 80 per cento per il Regno Unito), nonostante l'introduzione della riforma cosiddetta del $3+2$.

Eurostat, l'Ufficio Statistico della Comunità europea, ha recentemente rivisto l'indicatore, introducendo la dizione "Early level from education and training" rispetto al precedente "Early school leaver not in education or training". L'abbandono precoce dell'istruzione e della formazione, precedentemente indicato come abbandono scolastico, si riferisce a una persona di età compresa tra i 18 e i 24 anni che ha completato al massimo l'istruzione secondaria inferiore e non è coinvolta nell'ulteriore istruzione o formazione. L'indicatore "Early school leaver not in education or training" è espresso come percentuale delle persone di età compresa tra 18 e 24 anni con tali caratteristiche, rispetto alla popolazione totale dai 18 ai 24 anni. Rientrano in questo nuovo target i soggetti il cui livello più alto di istruzione o formazione è al massimo dell'istruzione secondaria inferiore (Livello 2 - International Standard Classification of Education) e che non hanno ricevuto alcuna istruzione o formazione (né formale né non formale) nelle quattro settimane precedenti l'indagine.

Il Comitato Nazionale per la Valutazione del Sistema Universitario (CNVSU) già nel 2011 aveva elaborato tre indicatori che facevano riferimento alla regolarità del percorso (percentuale di studenti regolari, o iscritti in corso), alla mancata produttività degli studenti (gli iscritti inattivi) e l'abbandono degli studi (percentuale di mancate re-iscrizione al II anno di corso) (cfr. Carci, 2013).

L'elaborazione degli indicatori di processo seguiva all'epoca un iter particolare: gli Uffici Statistici di ciascun Ateneo italiano trasmettevano i dati richiesti al MIUR, che dopo averli pubblicati, li metteva a disposizione dei Nuclei di valutazione delle singole Università. I Nuclei sottoponevano ad analisi e revisione i dati ricevuti dal MIUR e li mettevano a disposizione del CNVSU.

L’Agenzia Nazionale di Valutazione del Sistema Universitario e della Ricerca (ANVUR) a partire, invece, dal 2014, riprende le tre categorie utilizzate dal comitato ma cambiando le modalità di raccolta, intrecciando insieme l'analisi delle carriere attraverso i dati dell'Ufficio di Statistica del MIUR, l'analisi delle carriere attraverso i dati dell'Anagrafe Nazionale Studenti (ANS) e l'analisi degli indicatori per il Sistema Autovalutazione, Valutazione periodica, Accreditamento (AVA). Inattività, abbandono e ritardo sono anche gli indicatori presenti nell'ultimo Rapporto Biennale sullo Stato del Sistema Universitario e della ricerca prodotto alla fine del 2017 dall'AN- 
VUR e relativo al biennio 2014/2016. I dati relativi alla regolarità dei percorsi di studio mostrano un significativo miglioramento, sia per il numero di quanti terminano gli studi nei tempi previsti, sia con riferimento alla dinamica degli abbandoni precoci, ovvero della quota di immatricolati che non prosegue al secondo anno. Il tutto va letto tenendo conto del calo delle immatricolazioni che rappresenta probabilmente il primo elemento di selezione. Nell'anno 2014/2015 dopo undici anni dall'iscrizione risulta che il $57,8 \%$ degli studenti si è laureato, il 38,7\% ha abbandonato e il 3,5\% è ancora iscritto. Per contro, è da segnalare l'altissima percentuale (tra il $44 \%$ e il $48 \%$ in tutte le coorti) di studenti provenienti da un istituto professionale che dopo tre anni di corso triennale ha abbandonato l'Università e la marcata differenza geografica che vede al Nord i laureati regolari stabili per una quota di $38-40 \%$ mentre al Sud e nelle Isole è del $22-23 \%$. Tra coloro che cambiano corso, circa la metà effettua un trasferimento in un altro Ateneo. Nei corsi triennali, dopo quattro anni di corso, gli studenti che hanno effettuato un passaggio di corso dopo il primo anno hanno un tasso di successo molto simile a quello di coloro che rimangono nello stesso corso, osservati dopo tre anni (intorno al 35\%). Sempre a livello nazionale i laureati regolari stabili (nello stesso Corso di Studi di immatricolazione) sono il 31,9\% del totale, mentre analizzando i dati un anno oltre la durata del corso, i laureati stabili sono pari al $46 \%$.

A fianco degli abbandoni non sono trascurabili i passaggi di corso o di ateneo durante gli studi: i cambi di corso tra il primo e il secondo anno coinvolgono circa il $15 \%$ degli immatricolati nei corsi triennali e a ciclo unico, mentre sono molto contenuti nei corsi di laurea magistrale (sotto il $2 \%$ nelle tre coorti più recenti).

\section{Studenti con disabilità e con DSA all'Università}

Dall'entrata in vigore della legge n. 17 del 28 gennaio 1999, che integra e modifica la legge 104/1992, gli Atenei sono tenuti a garantire a tutti gli studenti in situazione di disabilità il pieno diritto allo studio e ad una più amplia inclusione sociale. Ogni istituzione universitaria è stata chiamata a nominare un docente delegato dal rettore con funzioni di coordinamento, monitoraggio e supporto delle iniziative a favore dell'inclusione, le cui azioni sono finanziate dal MIUR, a partire dal 1999, con un'apposita quota, utilizzando un fondo per il finanziamento ordinario delle università. 
La legge 17/1999 funge da punto di svolta tra due diverse modalità di intervento: prima del 1999, infatti, le azioni per l'inclusione avevano carattere sporadico, estemporaneo ed erano quasi sempre attivate come risposte a richieste specifiche; dopo l'emanazione della legge, l'intervento a favore degli studenti con disabilità diventa sistematico con l'apertura in quasi tutti gli Atenei italiani di un Servizio Studenti Disabili coordinato dal delegato del rettore alla disabilità e spesso affiancato da una Commissione Disabilità, con rappresentanti-referenti dei diversi Dipartimenti e finanziato dal MIUR in base al numero degli studenti disabili iscritti e ai progetti pianificati.

La legge 170 del 2010 "Nuove norme in materia di disturbi specifici di apprendimento in ambito scolastico" entra dopo dieci anni da questi provvedimenti, con lo scopo di garantire il diritto allo studio e di favorire il successo scolastico a tutti gli studenti con DSA. Nel gennaio 2010, con l'istituzione del Comitato Tecnico Scientifico con funzioni istruttorie per la predisposizione del decreto attuativo della legge 170, il Ministero ha richiesto la partecipazione della CNUDD, Conferenza Nazionale Universitaria Delegati Disabilità, che nel luglio del 2001 era stata nominata dalla Conferenza dei Rettori delle Università Italiane quale organo ufficiale di coordinamento delle università italiane a favore degli studenti con disabilità e/o con DSA.

La legge 170/2010 prevede, per la prima volta la possibilità di erogare agli studenti universitari con DSA specifiche misure di supporto e di introdurre strumenti compensativi, misure dispensative e adeguate forme di verifica e valutazione al fine di garantire il diritto allo studio di questi ragazzi.

È dunque previsto che ogni Ateneo offra loro diversi servizi, come quello di tutorato, l'attività di mediazione con il corpo docente e la possibilità di fruire degli strumenti utili allo studio individuale e al sostenimento degli esami curricolari.

Da una rilevazione Censis che ha coinvolto 40 Atenei (il 65\% delle università italiane), presentata nel capitolo "Processi formativi" del $51^{\circ}$ Rapporto sulla situazione sociale del Paese/2017 emerge che nell'anno accademico 2014-2015 gli studenti iscritti con disabilità (invalidità $>66 \%$ ) e con DSA sono stati 14.649: 10,2 studenti ogni mille. L'incremento è stato complessivamente del $+13,3 \%(+1,4 \%$ studenti con disabilità $\mathrm{e}+108,3 \%$ studenti con DSA).

Lo studente in situazione di disabilità e/o con DSA che si affaccia al percorso universitario è un giovane adulto chiamato a fare i conti con la propria identità (Contardi, 2004; Cottini, 2016; De Anna, 2014; Dettori, 2011; Goussot, 2009; Medeghini, 2006; Mura, 2014; Pavone, 2009; Arcangeli, 2017). L'esperienza universitaria è un banco di prova rispetto ai propri punti di forza 
ma anche in relazione a tutte le componenti deficitarie del proprio funzionamento. Un disturbo non è un elemento esclusivo e totalizzante del Sé, ma nemmeno una componente accessoria che è possibile tralasciare e non considerare in tutta la sua complessità.

Molti soggetti con disabilità e/o con DSA che hanno avuto una diagnosi in età infantile, se adeguatamente sostenuti e accompagnati in termini di opportunità e conferme, sono riusciti a riconoscersi dentro e fuori quella scrittura solo sanitaria, con possibilità di leggere il proprio disturbo in termini narrativi, di riconoscimento o estraniamento rispetto al quadro nosografico. Altri studenti, che non hanno avuto possibilità di percepirsi in termini di possibilità e di identità, non sono riusciti a ritrovare tracce di Sé dentro il quadro diagnostico e conclusa l'esperienza scolastica, dove spesso tutto è nato o si è manifestato, vorrebbero "ripartire da zero", fingendo e convincendosi che quel disturbo possa essere taciuto. L'esperienza universitaria, come potrebbe essere quella professionale o personale, finita la scuola, rappresenta una possibilità di ripartire da zero, risanando o almeno evitando il peso della sofferenza e della disconferma che batte ancora dentro. Questo desiderare di sperimentarsi da capo può condizionare fortemente la scelta del corso di studi e della professione, non più opzionato solo per desiderio e opportunità ma perché meglio consente di occultare la difficoltà, di fingersi non più marchiato e visibile. Il nascondere a sé e agli altri il proprio disturbo significa spesso essere disposti a pagare dei prezzi cognitivi ed emotivi altissimi, che si considerano più lievi rispetto a quanto il dichiarare comporterebbe. Molti studenti che hanno raggiunto la maggiore età senza aver ricevuto una diagnosi in età evolutiva possono sentire il bisogno di accedere ad alcuni diritti riconosciuti dalla recente normativa nel momento in cui si trovano ad affrontare l'esame di stato, il test di ammissione all'università, gli esami universitari o il mondo del lavoro. Proprio perché il riconoscimento di tali diritti è subordinato alla presentazione della diagnosi, la richiesta di una valutazione diagnostica da parte di adulti è in costante aumento.

Accompagnare uno studente con disabilità e/o con DSA verso il proprio personale progetto di vita significa in prima battuta aiutarlo a riconoscersi nella propria storia, qualunque essa sia, fare i conti con difficoltà e sconfitte, con potenzialità, compensazioni. Riconoscerne l'identità senza prendere per buona ciò che gli è stata attribuita è il primo passo per aiutarlo a riappropriarsi del proprio senso. A partire da questa nuova e continua ridefinizione di Sè, che cambia mano a mano che i vissuti diventano esperienze e le tracce personali elementi di conoscenza, è possibile attivare scelte consapevoli, fare i conti con il proprio personale progetto. Potrebbe esserci uno scarto considerevole tra l'idea e la possibilità, tra ciò che si pensa di voler scegliere ed 
essere in grado di realizzare e la realtà dei fatti. Il percorso che porta ogni persona a diventare consapevole dei propri punti di forza e dei propri limiti è già esso stesso esperienza di vita. Sbagliare, cambiare, riposizionarsi è diventare adulti, assumersi ovvero la responsabilità delle scelte. Aver cura del progetto di vita dell'altro non è solo «riparare le ferite, ma anche un far fiorire le possibilità dell'essere» (Mortari, 2016, p. 123) e scrivere i capitoli della propria esistenza significa allora aprirsi a tutte le dimensioni della propria cura, diventare attori delle proprie scelte, liberandosi dei retaggi e delle impossibilità sperimentate e diventate spesso una seconda pelle. Misurarsi con contesti che facilitano e altri che ostacolano consente di comprendere bene che non tutto dipende da sé: ogni progetto si dà in tempi e spazi definiti che possono essere disfunzionali, inadeguati, inaccessibili.

\section{Possibili interventi}

Dall'analisi del CENSIS, precedentemente citata, nella sezione dedicata al monitoraggio delle carriere, emerge come nell'A.A. 2014/2015 il 62,5\% degli studenti con disabilità e/o con DSA era in corso, mentre il $31,8 \%$ era fuori corso per un numero di anni non superiore al doppio della durata legale dello stesso. Il restante 5,7\% era irregolare per un numero di anni superiore al doppio della durata legale del corso stesso.

In particolare, interrogati sui possibili ambiti di miglioramento, gli studenti con disabilità partecipanti alla rilevazione ne hanno individuati principalmente tre: abbattimento delle barriere architettoniche, sistema di relazioni (rapporti con colleghi, docenti e altro personale universitario) e disponibilità di materiali didattici e di ausili tecnologici (Cfr. Pace, Pavone e Petrini, 2018). Per quanto riguarda gli studenti con DSA, le modifiche auspicate si concentrano soprattutto sulla dimensione relazionale-didattica: richiedono, infatti, maggiore disponibilità dei docenti verso le loro esigenze, un maggiore accesso al materiale didattico e al suo uso e una più ampia disponibilità di ausili tecnologici.

Inattività, abbandono e ritardo negli studenti con disabilità e/o con DSA sono spesso legati alla difficile relazione tra lo studente e il mondo accademico e alla tipologia di corso scelto. Un'analisi attenta del fenomeno e in particolare l'individuazione dei fattori di rischio, può essere utile per la definizione di una strategia di politiche di contenimento basate sul rafforzamento delle pratiche di orientamento e ri-orientamento, sul potenziamento del metodo di studio, sulla predisposizione di forme di tutorato e anche un ripensamento della didattica universitaria (Cfr. De Anna, 2016). In un'ottica 
di intervento inclusivo, a guadagnarne dovrebbe essere la qualità di tutta la proposta universitaria e, in maniera indiretta, la possibilità di contenere anche i dati e l'incidenza del fenomeno della dispersione degli studenti che vivono o non una situazione di disabilità e/o di svantaggio.

\subsection{Orientamento e ri-orientamento}

La scelta del corso universitario o di una possibile entrata nel mondo del lavoro rappresenta un nodo centrale per ogni studente. In entrambe le alternative c'è sempre un sogno-aspettativa che guida e orienta le decisioni. Una buona pratica di orientamento ha la funzione di connettere ciò che si pone idealmente come principio di piacere con un principio di realtà, che trae linfa dalle possibilità, bisogni, talenti, motivazioni degli studenti stessi e dal contesto. Nonostante negli ultimi anni molto si sia fatto in termini di orientamento scolastico, tutto questo non ha valore se non è collocato in una cornice di progressiva conoscenza e consapevolezza di sé, in termini di possibilità e fragilità.

Nel Decreto legislativo del 14 gennaio 2008 n. 21 si danno indicazioni alle scuole e alle istituzioni di istruzione superiore per la elaborazione di percorsi di orientamento per gli studenti dell'ultimo anno e per facilitare la loro scelta universitaria sulla base della conoscenza del mondo del lavoro, dei settori emergenti, dei percorsi di studio e per individuarne interessi e motivazioni specifiche. È condivisa la convinzione che le pratiche di orientamento devono poter consentire una crescente auto-consapevolezza in termini di progettualità personale, creando anche un'alleanza educativa con tutti $\mathrm{i}$ soggetti in causa. Si parla per questo di una didattica orientativa, azioni di tutorato e soprattutto di progettazione e di realizzazione di reti integrate, contestualizzate sul territorio, per l'orientamento.

La scelta dell' indirizzo di studi merita di essere valutata attentamente misurando con attenzione molteplici fattori, passioni, attitudini e potenzialità e considerando attentamente l'impegno necessario che ogni percorso comporta.

Molti studenti con disabilità e/o con DSA arrivano all'Università dopo un autentico percorso di orientamento e con un'adeguata maturità interna che permette loro una buona riuscita e un successo formativo in termini anche di progetto di vita. Per altri la scelta universitaria è invece figlia di logiche differenti, spesso legate alla necessità di rispondere ad un bisogno di altri, magari di chi si prende cura di loro. Riempire il vuoto successivo alla fine della scolarizzazione, in assenza di un dopo di noi degno di essere definito tale, 
può spingere molte famiglie a tentare il percorso accademico per posticipare lo scontro e il confronto con la realtà. In altri casi, in presenza di disabilità più invisibili, il percorso universitario nel suo poter sperimentare l'anonimato, può consentire il tentativo di cimentarsi in una realtà più "normale" possibile, meno vittima di stereotipi e pregiudizi. In altri casi il ri-orientamento diventa necessario: il percorso intrapreso paga il prezzo di scelte altrui, di solito in buona fede, che sembrano però lontane dalle possibilità e dalle motivazioni degli studenti stessi.

In termini di ri-orientamento l'obiettivo è quello di diventare consapevoli delle proprie mappe e del proprio personale modo di interpretare la realtà, diventare attori di scelte vicine ai bisogni e ai desideri, rimanendo disponibili a negoziare e risignificare i propri orizzonti personali e professionali (Batini e Del Sarto, 2005; Batini e Giusti, 2008).

Accompagnare uno studente in un percorso di orientamento, lavorare per le sue «competenze di autorientamento, con la finalità di scelte (il plurale è d'obbligo) immediate o future, in direzione di una decisione o per una lettura più appropriata di un contesto esistenziale e/o professionale, per progettare un percorso formativo o per migliorare la percezione di sé in direzione di un'efficacia maggiore nell'azione di soddisfacimento dei propri bisogni, di realizzazione dei propri progetti e desideri, in direzione di una maggiore chiarezza su questi stessi» (Batini e Del Sarto, 2007, p. 48). Si tratta di focalizzare l'attenzione sulla storia di ogni studente, le sue percezioni, i suoi vissuti, il suo modo di vivere e significare. La sfida dell'orientamento è consentire allo studente di imparare come scegliere, piuttosto che fare la scelta giusta. Per poter lavorare in termini di deuteroapprendimento, è necessario porsi in un'ottica narrativa che rispetta le variabili temporali e contesuali. La letteratura sistemico-costruttivista legata al counselling (Boscolo e Bertrando, 1993; Cecchin e Apolloni, 2003) ha evidenziato come si tratta di portare a maturare la consapevolezza che nessuna pratica di orientamento dovrebbe ridurre il campo ad un aut aut ma essere sempre un'amplificazione di possibilità. «Come accade nei processi evolutivi, in un mercato incerto le persone si orientano in relazione di adattamento evolutivo con i contesti: scelgono e attivano alcune competenze prima orientate in altri settori o latenti. In tal senso, il celebre dubbio "scelgo tra passione o dovere?" si può trasformare nella domanda "come posso far convivere passione e dovere?", poiché nell'incertezza non c'è spazio per una sola scelta, definitiva e certa, quanto piuttosto per un re-inventarsi ciclico che favorisce l'attivazione delle competenze utili in situazione. Passione e dovere divengono così entrambi luogo di risorse e attivatori di competenze spendibili nel presente o nel futuro, e non elementi tra cui scegliere in via definitiva» (Vitale, 2012, p. 87). 
Ri-orientare significa allora partire da una riflessione sui dispositivi in campo nelle prassi d'orientamento e sui processi di apprendimento che questi favoriscono e consentire la consapevolezza e la messa in discussione delle mappe che orientano ogni studente nelle scelte.

\subsection{Metodo di studio}

L'ingresso nel mondo universitario rappresenta spesso la prova del nove rispetto al proprio metodo di studio, messo a punto negli anni scolastici. Di fatto con l'adozione e lo sviluppo del sistema dei crediti ECTS (European Credit Transfer System), l'attenzione si focalizza non più sul docente e sulle sue ore d'insegnamento, ma sullo studente e sul suo carico di lavoro (composto da ore di lezione, ore di laboratorio e ore di studio): diventa importante di conseguenza riflettere sui suoi processi di apprendimento e sul processo di insegnamento/apprendimento, che è quindi ora sempre più centrato sullo studente.

Lo studio è sicuramente un'attività complessa che intreccia insieme variabili cognitive, metacognitive ed emotivo-relazionali (Cfr. De Beni, Moè e Cornoldi, 2003; Cornoldi et al., 2005). Il metodo di studio ha quindi a che fare con la motivazione, l'organizzazione della attività di studio, le strategie di comprensione e la memoria, ma anche con una crescente consapevolezza del proprio stile cognitivo e dell'approccio messo in campo fino a questo momento.

Gli studenti con disabilità che arrivano all'università hanno sperimentato nel loro percorso di studi l'affiancamento del docente specializzato, un sostegno che dovrebbe essere modulato in base alla specificità del deficit e alla disponibilità delle risorse del contesto scuola. Al di là delle ore di cui si è usufruito, non sempre l'esperienza del sostegno è occasione di crescita in termini di autonomia. Spesso, la delega dell'esperienza, trasforma l'intervento in pratiche di cura scarsamente autentiche, dove a prevalere sono interventi di sostituzione, iperprotezione e anticipazione che non facilitano l'acquisizione di un metodo consolidato ed autonomo.

Le difficoltà possono manifestarsi nel prendere appunti, nel rielaborarli, nell'approcciarsi ad un testo in maniera completa o nella definizione dei tempi di studio e/o ripasso in vista dell'esame stesso.

L'organizzazione dell'attività di studio, che è comunque profondamente diversa tra scuola ed università per tutti gli studenti, è spesso un momento critico: pianificare obiettivi, tempi e modalità di studio è un compito inedito per molti. «Uno studente ben organizzato sa alternare in modo equilibrato 
impegni e svaghi, conosce con esattezza il proprio tempo a disposizione per studiare, evita la preparazione affrettata dell'ultimo momento, conosce la propria velocità di lettura e comprensione, sa usare e organizzare il tempo di cui dispone, secondo le personali esigenze e gli impegni che si è prefissato (Legrenzi, 1994)» (De Beni et al., 2015, p. 16).

Facendo seguito al documento emerso dal progetto "Higher Education Accessibility Guide" promosso dall'European Agency for Development In Special Needs Education e alle linee guida prodotte dalla CNUDD (2014), molti Atenei hanno preso in mano la sfida investendo su attività di tutorato specializzato e alla pari e su servizi di counseling al fine di sostenere lo studente in questo percorso di messa a punto e sostegno del metodo.

\subsection{Didattica universitaria}

Sicuramente buona parte degli studenti con disabilità e/o con DSA che arrivano all'Università hanno già nel precedente percorso scolastico individuato strategie compensative e modalità di apprendimento, che hanno permesso la costruzione di una fiducia verso il sistema-scuola.

La loro presenza può rappresentare per un docente universitario una sfida significativa. Ad essere messa in discussione è infatti la modalità tradizionale e consuetudinaria di organizzare l'insegnamento, proporre i contenuti ma al tempo stesso anche di valutare gli apprendimenti. La didattica universitaria comincia da poco ad interrogarsi su alcuni elementi della sua proposta, sulla diversità di tempi e di apprendimento dei singoli studenti, sull'opportunità di non eccedere nella durata o nella quantità di informazioni da trasmettere durante le singole lezioni. Siamo comunque sempre di fronte ad un sistema di istruzione spesso più prestazionale che formativo, dove a prevalere è la postura cognitiva-performativa.

L'Associazione Italiana Editori e Fondazione Agnelli hanno promosso, per la prima volta in Italia, una ricerca su questo tema, che è stata presentata nel settembre 2016. Dalla ricerca emerge come manca nella totalità dei docenti intervistati, una consapevolezza di come il proprio insegnamento contribuisca al raggiungimento dei risultati di apprendimento attesi del corso di studio nel quale l'insegnamento è inserito, ma in alcuni casi anche ostilità e fastidio per questa tipologia di strumenti che in qualche modo sono accusati di limitare l'autonomia didattica. I docenti appaiono consapevoli dell'importanza dei metodi didattici e di come l'utilizzo di differenti approcci influisca sul processo di apprendimento. A fronte di queste consapevolezza viene meno una considerazione sistemica (soprattutto a livello di corso di studio) 
relativa a quali metodi siano più adeguati per rispondere al meglio al progetto formativo proposto e con i colleghi sono rare le occasioni di confronto e scambio. Molto forte, viceversa, risulta l'influenza della disciplina sull'impostazione metodologica e questo legame, in alcuni casi, sembra indurre una certa conservazione dei metodi didattici. Gli intervistati confermano una maggiore enfasi sulla ricerca rispetto alla didattica, anche in ragione della VQR. Il corpo docente è spesso autonomamente sensibile ai temi dell'apprendimento, ma a mancare è una vera e propria consapevolezza didattica, che richiederebbe investimenti importanti in formazione nelle fasi iniziali della carriera e momenti di aggiornamento e potenziamento delle competenze didattiche in itinere, coinvolgendo con forza le comunità disciplinari. Ad essere ripensati, come emerge dalla ricerca, dovrebbero essere anche $\mathrm{i}$ meccanismi incentivanti del corpo docente che attribuiscano un peso significativo alla sfera didattica, incentivando l'impegno in innovazione, organizzazione e coordinamento.

Spesso i docenti non possiedono sufficienti conoscenze rispetto ai diversi deficit o disturbi, ma nemmeno sufficienti chiavi ermeneutiche per leggere oltre una diagnosi. Ogni studente ha un proprio profilo di funzionamento che non necessariamente coincide con l'immaginario studiato o costruito rispetto alla letteratura. «Nello specifico, i docenti che si confrontano direttamente con il problema, quando esso è ormai strutturato, avvertono il bisogno di ampliare la propria formazione attraverso incontri con esperti o partecipando a convegni e richiedendo il supporto di altre professionalità per poterlo affrontare. Sarà importante conoscere la specificità del disturbo, ma soprattutto considerare l'individuo nella sua unicità» (Guaraldi, Pedroni e Fantera, 2010, p. 165). Nessuna conoscenza può costruirsi senza un incontro: concedersi uno spazio di reciproco ascolto rispetto alla propria proposta didattica e alla specificità di bisogni e risorse che ogni studente può rappresentare, è un'occasione importante per costruire gradualmente una nuova cultura didattica. Altra alleanza da costruire è quella con il mondo della scuola secondaria, che può regalare una storia importante in termini di mediazioni e strategie già sperimentate e collaudate. Il mondo universitario non rappresenta l'anno zero: è possibile ritrovare tra le tracce del percorso scolastico indizi importanti sia sul piano della proposta dell'accessibilità dei contenuti che delle forme di compensazione e/o dispensazione. «Le transizioni tra cicli e tra contesti scolastici possono fornire elementi di studio interessanti anche per comprendere le dinamiche del successo e dell'insuccesso scolastico, in quanto le opportunità di confronto rendono più visibili, sia all'individuo che le vive sia al docente o ricercatore che le voglia comprendere, le complesse dinamiche di interazione tra soggetto e contesto» (Bonica, 2013, p. 56). 
Dentro una didattica inclusiva «gli interventi di cura educativa speciale diventano ordinari, a beneficio di tutti, secondo un approccio multidimensionale, teso a rimuovere gli ostacoli all'apprendimento e alla partecipazione» (Carlini, 2015, p. 75).

Dentro questa cornice oggi trova spazio l'approccio dell'Universal Design for Learning promosso dal gruppo di ricercatori del CAST, pubblicato prima nel 2008 (versione 1.0) e poi nel 2011 (versione 2.0).

La progettazione universale ha lo scopo di individuare una serie di principi che consentono la costruzione di un curriculum che sappia garantire pari opportunità apprenditive e al tempo stesso permettere a tutti gli studenti di sperimentarsi come attori competenti. Di fatto l'UDL fornisce un modello per la creazione di obiettivi, metodi, materiali e strumenti di valutazione che nascono non in una sola dimensione, ma già al plurale, approcci fluidi e flessibili che prendono forma in base ai bisogni e alle capacità dei soggetti a cui si rivolgono. Supera la questione dell'accessibilità a posteriori dei processi di apprendimento-insegnamento per alcune categorie di alunni, evidenziando come ogni didattica che prevede "un unico livello" innalza involontariamente le barriere dell' apprendimento per tutti. A cambiare è la direzione dell'intervento che non chiede agli studenti di adattarsi ad una proposta ma alla pratica didattica di tingersi di accessibilità, riconoscendo come ogni forma di catalogazione e classificazione degli studenti in base a stili cognitivi, bisogni e/o interessi di fatto limita l'orizzonte potenziale degli apprendimenti. L'orizzonte inclusivo si apre dentro questa cornice all'importanza dell'accessibilità del sapere, alla qualità dell'esperienza di apprendimento e al successo formativo di tutti. Lo scopo è quello di formare studenti esperti ben informati e pieni di risorse, determinati e motivati, strategici ed orientati agli obiettivi.

L'approccio intreccia ricerche recenti in ambito neuroscientifico con alcuni studi in ambito psicopedagogico che ormai da tempo, in ottica costruttivista, sostengono la centralità del riconoscimento della variabilità soggettiva negli apprendimenti, frutto di un equilibrio dinamico bio-psicosociale e contestuale.

Partendo da questi studi, l'Universal Design for Learning va ad individuare le reti celebrali interconnesse che intervengono nell'apprendimento: la rete di riconoscimento (il che cosa), la rete strategica (il come) e la rete affettiva (il perché).

Da queste reti derivano i principi elaborati dal CAST:

- Fornire molteplici mezzi di rappresentazione che riguarda il che cosa dell'apprendimento, le differenti modalità in cui è percepita e compresa 
l'informazione. Ne deriva la necessità di fornire opzioni alla percezione, alla lingua e ai simboli e alla comprensione.

- Fornire molteplici mezzi di azione e di espressione che riguarda il come dell'apprendimento, il «modo in cui gli individui affrontano le sfide dell'apprendimento ed esprimono la loro conoscenza» (Rose e Gravel, 2016, p. 35). L'opzione di cui si parla può essere legata all'azione fisica, alle abilità espressive e fluidità e alle funzioni esecutive.

- Fornire molteplici mezzi di coinvolgimento che implica una riflessione sulle differenti motivazioni all'apprendimento. Questo principio si declina nella possibilità di fornire opzioni per suscitare interesse, per attivare uno sforzo sostenibile e la perseveranza, per favorire l'autoregolazione.

La proposta dell'Universal Design risponde ad esigenze che emergono in significativi contributi che oggi chiedono alla didattica di differenziarsi in termini di contenuti, processi e prodotti (Tomlinson, 1999; D'Alonzo, 2016) a partire dal sapere e dalla competenza personale degli studenti, dalla loro motivazione, dal bisogno di autodeterminazione e di relazione. L'UDL sposa una concezione sistemica e costruttivista della relazione insegnamento-apprendimento dove successo o fallimento non dipendono solo dalle caratteristiche personali e dall'impegno e approfondimento dello studente, ma in maniera autocritica anche alla proposta didattica di lavoro, alla relazione e al contesto dentro i quali i saperi sono stati condivisi. La sfida da accogliere è quella di andare oltre la semplice acquisizione di contenuti e nozioni, per affrontare la possibilità di ricercare e costruire conoscenze a partire dalla relazione tra saperi e linguaggi specifici disciplinari maggiormente spendibili in termini di apprendimenti significativi e professionalizzanti. Tutto questo si gioca nella possibilità di orchestrare insieme accessibilità e condivisione, consapevolezza e continuità. L'approccio Scaffolded Knowledge Integration (Linn, Davies e Bell, 2004) dentro questa cornice ha evidenziato l'importanza di rendere accessibili i saperi ma al tempo stesso di rendere visibile il pensiero, aiutare gli studenti a cogliere le connessioni tra le idee con il fine di formare nuove reti di connessione; aiutare gli studenti ad ascoltare e ad apprendere dai pari in modo collaborativo, promuovendo forme di apprendimento autonomo e permanente.

«L'UDL affronta la sfida di attuare un'educazione valida per tutti proponendo criteri attuativi concreti. Una semplice lettura è sufficiente per mostrare come in quelle linee guida si coniughi l'istanza volta ad assicurare una pluralità di risorse e di codici comunicativi, come strumento a cui ricorrere per venire incontro alla specificità del soggetto, con i principi base dell'istruzione» (Calvani, 2012, p. 86). 
Tutto questo vale anche per le strutture universitarie chiamate così come cita la Convenzione sui diritti delle persone con disabilità (2006), ad una «progettazione di prodotti, strutture, programmi e servizi utilizzabili da tutte le persone, nella misura più estesa possibile, senza il bisogno di adattamenti o di progettazioni specializzate», prevedendo anche «dispositivi di sostegno per particolari gruppi di persone con disabilità ove siano necessari» (art. 2).

\section{L'esperienza dell'Università di Perugia}

Nell'Ateneo di Perugia gli studenti con disabilità e/o DSA degli ultimi dieci anni risultano essere n. 1101. Di questi n. 177 studenti hanno dichiarato di voler rinunciare agli studi; n. 65 studenti hanno poi ripreso e 8 di loro si sono iscritti al medesimo corso di studio.

L'Università di Perugia ha scelto di mettere a disposizione sostegni e mediatori di prossimità per evitare i rischi di dipendenza, forme di vittimismo $\mathrm{e}$ richieste risarcitorie, che evolvono con lo studente, divenendo mano a mano meno invasivi per consentire l'appropriarsi di pratiche di empowerment e di auto-organizzazione.

Dovendo attivare una serie di servizi per rispondere a questi bisogni ci si è trovato di fronte ad un bivio di carattere metodologico e politico: optare per un sentiero "tecnico" che procede per categorie e per specialismi o per uno più "prassico" che privilegia un pensiero che parte dai vissuti e dalle storie per attivare di volta in volta procedure contestualizzate ed individualizzate (Arcangeli, 2005; Sannipoli, 2015). La scelta del secondo approccio è stata quella ritenuta più rispettosa e inclusiva per gli studenti iscritti, con la consapevolezza che aver cura del percorso di studi significa non tanto propiziare un passaggio verso la vita adulta ma trovare il modo di rendere più felice e significativo quel soggiorno, potendo sperimentare spazi crescenti di autonomia, libertà e autodeterminazione.

In questo senso negli ultimi anni sono state fatte molte azioni di sostegno in favore di studenti con disabilità e/o con DSA, che trova una sua legittimazione nel nuovo Regolamento di Ateneo per l'inclusione e il diritto allo studio degli studenti con disabilità e/o DSA approvato nel dicembre 2017.

In collaborazione con il Servizio di supporto, si è intensificata l'attività di tutorato specializzato, affiancata da un crescente investimento nel tutorato alla pari, sperimentato ormai dal 2017, con risultati ottimi. Essere accompagnanti da un proprio collega, dove il funzionamento dello studente lo consenta, è occasione di crescita non solo in termini di apprendimento, ma anche di "vita", che evitano ulteriori esperienze di delega e/o di cura inautentica. 
L'esperienza, accompagnata da una formazione iniziale e in itinere e continuamente supervisionata, rappresenta proprio la dimostrazione della possibilità di realizzare interventi coevolutivi che fanno crescere cultura e al tempo stesso confermano la centralità di approcci basati sull'apprendimento situato e cooperativo (Cfr. Mitchell, 2016).

Sono stati progettati e realizzati due servizi aperti a tutti gli studenti dell'Ateneo, ma con una competenza specifica per gli studenti con disabilità e/o con DSA: il servizio FOCUS e il laboratorio Inl@b.

FOCUS è servizio a due facce: uno di Counseling Psicologico e uno Pedagogico-didattico. Il servizio psicologico è uno spazio di ascolto e di sostegno per tutti gli studenti iscritti all'Ateneo di Perugia volto alla gestione di problematiche personali, relazionali, emotive, ansia, dubbi sulle proprie capacità personali legate allo studio universitario che possono emergere durante il percorso accademico. Sulla base del primo colloquio il Servizio non prende in carico i casi che richiedono trattamenti d'urgenza o in cui siano necessarie impostazioni di setting specifici. Il servizio pedagogico-didattico è uno spazio di ascolto e di relazione d'aiuto per tutti gli studenti iscritti all'ateneo di Perugia volto a fornire un sostegno nell'affrontare le problematiche relative ai processi di apprendimento Lo scopo è offrire sostegno nell'acquisizione e/o affinamento della metodologia di studio, nella ridefinizione del proprio percorso di studio in termini di ri-orientamento; nella lettura dei bisogni specifici in direzione della delineazione del Progetto di vita per gli studenti con disabilità, nell'individuazione di strumenti compensativi per gli studenti con DSA. In entrambi i casi l'intervento è limitato nel tempo e prevede un follow-up per coglierne l'efficacia.

Accanto al servizio di Counseling, è stato predisposto un Laboratorio di tecnologie inclusive InL@b, uno spazio, attrezzato di tecnologie dell'informazione e della comunicazione (TIC) per supportare i momenti di studio e di ricerca personali degli studenti. Le TIC, qualora necessario, possono essere date in comodato d'uso sia allo studente che al Dipartimento che ne fa richiesta.

Sono state predisposte linee guida sui DSA per il personale docente e per il personale $\mathrm{TAB}$ con lo scopo, insieme ad alcuni interventi formativi e di disseminazione scientifica, di favorire la costruzione di una cultura più diffusa sua queste tematiche; ad ogni studente con DSA è stata consegnata una chiavetta USB con software gratuiti per la lettura e scrittura.

La costruzione di una sezione del sito dedicata ed accessibile, il monitoraggio delle barriere architettoniche, la predisposizione di accordi per il rinnovo delle diagnosi in caso di studenti con DSA che arrivino con documentazioni non aggiornate e di incontri con le associazioni no profit del territorio 
per spostamenti e assistenza, la possibilità di dotare i Dipartimenti di kit per una didattica che arriva anche a chi non può muoversi dalla propria residenza, sono tra le ultime sfide messe in campo per permettere a questi studenti di stare con diritto nei percorsi universitari.

Coerentemente con le premesse teoriche sopra descritte, si sta concludendo anche un progetto di ricerca che si è proposto di indagare i bisogni evolutivi degli studenti con disabilità e/o con DSA e il processo di costruzione del Sé nel giovane adulto, valutando i profili di funzionamento degli studenti in termini di autostima, autonomia, autoconsapevolezza e resilienza.

La ricerca sta coinvolgendo tutti gli studenti dell'Università degli Studi di Perugia con disturbi specifici dell'apprendimento (n. 88) e studenti con disabilità (n. 204) con un grado di invalidità superiore al $66 \%$ che si sono autosegnalati al momento dell'iscrizione.

Alla luce della complessità dell'oggetto di studio, la dimensione intra e interpersonale nella costruzione del Sé nel giovane adulto, si è scelto di adottare un approccio multi-method (Waszak e Sines, 2003) che coniuga uno studio quantitativo, attraverso strumenti self-report, a uno studio qualitativo mediante intervista. Dopo la compilazione del questionario anagrafico, la Rosenberg Self-Esteem Scale (RSER), il Questionario Ansia e Resilienza (QAR), la Self-Consciousness Scale (SCS-R), l'Identity Stile Inventory-5 (ISI-5) e infine il Questionario sulle Strategie di Studio (QSS). viene condotta la Life Interview (McAdams, 2001, 2012), un'intervista semi-strutturata che rileva la narrative identity (o narrative self). La life interview, per la sua forma semi-strutturata e che accompagna gli studenti a ripercorrere i capitoli della propria storia, incoraggia la persona a parlare di sé in modo libero e autentico, promuovendo un processo di auto-esplorazione e di riflessione su motivazioni più profonde che sottendono ai propri comportamenti, idee e decisioni, motivazione intrinseca.

Uno studente con disabilità e/o con DSA che arriva all'università porta con sé un vissuto che non è solo un bisogno ma che è anche domanda che irrompe nell'ordinario e chiede al mondo accademico di essere altro e di andare oltre le tradizionali forme e vie percorse. Ancora una volta la "specialità" di questi studenti può essere una rinnovata occasione per ripensare la proposta comune, ordinaria, che raffinando la sua forma, ne esce profondamente arricchita. 


\section{Riferimenti bibliografici}

ANVUR (2014), Rapporto sullo stato del sistema universitario e della ricerca 2013, Roma, testo disponibile al sito: http://www.anvur.org/attachments/article/644/Rapporto\%20ANVUR\%202013_UNIVERSITA\%20e\%20RICERCA_integrale.pdf.

ANVUR (2017), Rapporto sullo stato del sistema universitario e della ricerca 2016, Roma, testo disponibile al sito: http://www.anvur.org/index.php?option=com_content $\&$ view $=$ article $\& i d=1045 \&$ Itemid $=708 \&$ lang $=i t$ ).

Arcangeli L. (2005), Il silenzio come possibilità per una didattica speciale, Morlacchi, Perugia.

Arcangeli L., a cura di (2017), Studenti con DSA. Pratiche di empowerment all'Università, Carocci, Roma.

Balduf M. (2009), "Underachievement Among College Students", Journal of Advanced Academics, 20, 2: 274-294.

Batini F. e Bartolucci M., a cura di (2016), Dispersione scolastica. Ascoltare i protagonisti per comprenderla e prevenirla, FrancoAngeli, Milano.

Batini F. e Del Sarto G. (2007), Raccontare storie. Politiche del lavoro ed orientamento narrativo, Carocci, Roma.

Batini F. e Del Sarto G. (2015), Narrazioni di narrazioni. Orientamento narrativo e progetto di vita, Erickson, Trento.

Batini F. e Giusti S. (2008), L'orientamento narrativo a scuola. Lavorare sulle competenze per l'orientamento dalla scuola dell'infanzia all'educazione degli adulti, Erickson, Trento.

Benvenuto G. e Carci G. (2008), "La dispersione universitaria: indicatori nazionali e modelli di analisi longitudinale alla Sapienza, Università di Roma", Psicologia dell'educazione e della formazione, SIPEF, 10, 2: 5-46.

Benvenuto G. e Carci G. (2010), "Passaggi di corso degli studenti e orientamento all'università: uno studio sull'Università Sapienza di Roma”, Giornale Italiano della Ricerca Educativa, 3, 4: 9-20.

Berger J.B. and Lyons S.C. (2005), Past to Present: A Historical Look at Retention, in Seidman A., ed., College Student Retention: Formula for Student Success, 1-29, Praeger Publishers, Westport, CT.

Bonica L. (2013), Transizioni ecologiche e visibilità dei contesti, in Ligorio M.B. e Pontecorvo C., a cura di, La scuola come contesto. Prospettive psicologiche-culturali, Carocci, Roma.

Boscolo L. e Bertrando P. (1993), I tempi del tempo. Una nuova prospettiva per la consulenza e la terapia sistematica, Bollati Boringhieri, Torino.

Calvani A. (2012), Per un 'istruzione evidence based. Analisi teorico-metodologica internazionale sulle didattiche efficaci e inclusive, Erickson, Trento.

Carci G. (2013), Orientamentoring: I servizi di supporto agli studenti universitari per contrastare la dispersione, Libellula, Tricase (LE).

Carlini A. (2015), BES in classe. Modelli didattici e organizzativi, Tecnodid, Napoli.

Cecchin U. e Apolloni T. (2003), Idee perfette. Hybris delle prigioni della mente, FrancoAngeli, Milano.

CENSIS (2017), $51^{\circ}$ Rapporto sulla situazione sociale del Paese/2017, Roma.

CNUDD (2014), Linee Guida, Bergamo, testo disponibile al sito: http://www2.crui.it/crui/cnudd/Llinee_guida_CNUDD/LI-

NEE_GUIDA_CNUDD_2014.pdf. 
CNVSU (2011), Undicesimo Rapporto sullo Stato del Sistema Universitario, Roma, testo disponibile al sito: http://www.cnvsu.it/_library/downloadfile.asp?id=11778).

Colombo M. (2010), Dispersione scolastiche e politiche per il successo formativo: Dalla ricerca sugli early school leaver alle proposte di innovazione, Erickson, Trento.

Contardi A. (2004), Verso l'autonomia. Percorsi educativi per ragazzi con disabilità intellettiva, Carocci, Roma.

Cornoldi C., De Beni R., Zamperlin C. e Meneghetti, C. (2005), Amos 8-15. Abilità e motivazione allo studio: prove di valutazione per ragazzi dagli 8 ai 15 anni, Erickson, Trento.

Cottini L. (2016), L'autodeterminazione nelle persone con disabilità. Percorsi educativi per svilupparla, Erickson, Trento.

D’Alonzo L. (2016), La differenziazione didattica per l'inclusione, Metodi, strategie, attività, Erickson, Trento.

De Anna L. (2014), Pedagogia speciale. Integrazione e inclusione, Carocci, Roma.

De Anna L. (2016), Teaching accessibility and inclusion, Carocci, Roma.

De Beni R., Moè A. e Cornoldi C. (2003), Amos. Abilità e motivazione allo studio: prove di valutazione e orientamento, Erickson, Trento.

De Beni R., Zamperlin C., Fabris M. e Mereghetti C. (2015), Studiare meglio e riuscire all'università. Linee guida e materiali per potenziare le abilità di studio, Erickson, Trento.

Dettori F. (2011), Perdersi e ritrovarsi. Una lettura pedagogica della disabilità in età adulta, FrancoAngeli, Milano.

Di Pietro G. and Cutillo A. (2008), "Degree flexibility and university drop-out: The Italian experience", Economics of Education Review, 27: 5456-555.

European Agency for Development in Special Needs Education (2005), Higher Education Accessibility Guide, Odens C.

Garza E. and Bowden R. (2014), "The Impact of a First Year Development Course on Student Success in a Community College: An Empirical Investigation", American Journal of Educational Research, 2.6: 402-419.

Goussot A., a cura di (2009), Il disabile adulto. Anche i disabili diventano adulti e invecchiano, Maggioli, Santarcangelo di Romagna.

Guaraldi G., Pedroni P. e Moretti Fantera M. (2010), Al diploma e alla laurea con la dislessia. Storie di vita e metodologie per la scuola secondaria di secondo grado e l'università, Edizioni Centro Studi Erickson, Trento.

Heublein U., Hutzach C., Schreiber J., Sommer D. and Besuch G., (2010), "Ursachen des Studienabrruchs in Bachelor- und in herkömmlichen Studiengängen. Ergebnisse einer Bundesweiten Befragung von Exmatrikulierten des Studienjahres 2007/2008", HIS: Forum Hochschule, 2.

Heublein U., Spangenberg H., Sommer D. (2003), "Ursachen des Studienabbruchs: Analyse 2002", Hochschulplanung, HIS, Hannover.

Linn M.C., Davis E.A. and Bell P. (2004), "Inquiry and technology", Internet environments for science education, 3-28.

McAdams D.P. (2001), “The psychology of life stories”, Review of General Psychology, 5: 100-122.

McAdams D.P. (2012), "Exploring psychological themes through life-narrative accounts", Varieties of narrative analysis, 3: 15-32.

McCall R.B. (1994), “Academic Underachievers”, Current Directions in Psychological Science 3, 1: 15-19.

Medeghini R., a cura di (2006), Disabilità e corso di vita. Traiettorie, appartenenze e processi di inclusione delle differenze, FrancoAngeli, Milano.

Mitchell D. (2016), Diversities in education: Effective ways to reach all learners, Routledge, 
London, NY.

Mortari L. (2016), Filosofia della cura, Raffaello Cortina, Torino.

Mura A. (2014), Diversità e inclusione. Prospettive di cittadinanza tra processi storico- culturali questioni aperte, FrancoAngeli, Milano.

Pace S., Pavone M. e Petrini D., a cura di (2018), UNIversal inclusion. Rights and opportunities for students with disabilities in the academic context, FrancoAngeli, Milano.

Pavone M., a cura di (2009), Famiglia e progetto di vita. Crescere un figlio disabile dalla nascita alla vita adulta, Erickson, Trento.

Rose D.H e Gravel J.W. (2016), Universal Design for Learning: un 'introduzione per l'Italia, in Savia G., a cura di, Universal Design for Learning. Progettazione universale per l'apprendimento e didattica inclusive, Erickson, Trento.

Sannipoli M. (2015), Diversità e differenze nella prospettiva coevolutiva, FrancoAngeli, Milano.

Tomlinson C.A. (1999), The differentiated classroom: responding to the needs of all learners, ASCD, Alexandria, VA.

Vitale A. (2012), Metodologie trasformative. Lavorare con operatori e famiglie nei servizi educativi, Guerini Scientifica, Milano.

Waszak C. and Sines, M.C. (2003), Mixed methods in psychological research, in Tashakkori A. and Teddlie C., eds., Handbook of mixed methods in social and behavioral research, Sage, Thousand Oaks, CA. 


\section{Parte II}

\section{Pratiche e politiche per prevenire la dispersione}




\title{
Se me ne vado, ci saranno problemi... \\ La mancanza di adesione alle offerte formative in Danimarca, in una prospettiva di capabilities ${ }^{1}$
}

\author{
di Christian Christrup Kjeldsen
}

If I go, there will be trouble. And if I stay it will be double

The Clash

\section{Introduzione}

I sistemi educativi giusti possono definirsi in modi diversi, a seconda della propria idea di giustizia (Sen, 2009). Queste differenti idee di giustizia sollevano alcune questioni di uguaglianza nell'educazione, per nominarne alcune: La politica educativa punta principalmente a fornire pari opportunità o uguali risultati? Queste opportunità sono considerate come diritti formali o piuttosto come libertà reali per «raggiungere quelle cose a cui [i giovani] hanno ragione di dare valore» (Sen, 2009, p. 231)?

L'uguaglianza nell'educazione è stata una delle questioni principali della sociologia e della politica educativa a partire dalla metà degli anni ' 60 , spesso tuttavia è stata formulata come preoccupazione legata agli investimenti nel capitale umano e al ritorno di tali investimenti, alimentata dall'idea di una "riserva di intelligenze" che dovrebbe essere capitalizzata (Kjeldsen, 2016).

\section{Alunni svantaggiati - una cartina di tornasole per valu- tare i sistemi educativi in termini di giustizia sociale}

Se si dovesse giudicare - da un punto di vista di giustizia sociale - un sistema educativo, il responso potrebbe apparire piuttosto chiaro guardando alle politiche inerenti gli studenti più svantaggiati, provenienti da famiglie con uno status socio-economico basso. Qual è la loro libertà di vivere la vita a cui attribuiscono valore? Quali scelte vengono date - se ne vengono date?

\footnotetext{
${ }^{1}$ La traduzione in italiano del contributo è a cura di Marco Bartolucci e Irene D.M. Scierri. In appendice viene presentato un approfondimento sul sistema scolastico danese.
} 
Adottando la chiave di lettura di Bourdieu, questi studenti, spesso provenienti da famiglie di basso status culturale ed economico-sociale, fanno parte della porzione dominata della società, entro cui la scuola svolge un importante ruolo di riproduzione delle strutture di dominio e potere e di vantaggi e svantaggi, da una generazione all'altra (Bourdieu e Passeron, 1990; Bourdieu, 2007 [1979]). Da questa prospettiva, un'analisi dei risultati della scuola, in relazione alle opportunità educative, può essere intesa come un'analisi che «mette in evidenza la contraddizione tra quella verità oggettiva e la pratica degli agenti, che oggettivamente manifesta il misconoscimento di quella stessa verità» (Bourdieu e Passeron, 1990, p. 12).

Nel caso qui in esame, la verità oggettiva in Danimarca è che l'istruzione superiore è un'opportunità aperta a tutti. Tutti gli studenti che entrano nel sistema di istruzione sono, infatti, nella stessa condizione e hanno diritto al sostegno economico mentre studiano. La comune verità "oggettiva", presente nel discorso pubblico, diventa quindi: "in Danimarca ogni giovane ha le stesse opportunità gratuite per l'istruzione". Persino il rispettato studioso Thomas Piketty ha sostenuto in un discorso pubblico alla London School of Economics che questo è uno dei motivi principali dell'elevata equità trovata nei paesi nordici (Rodgers, 2014). Se si sottoscrive l'idea della giustizia come opportunità formali di parità, il sistema educativo danese sembra piuttosto giusto e noi tutti possiamo dormire sogni traquilli. Tuttavia, è possibile chiedersi: che realtà emerge se noi, invece dei diritti formali, ci concentriamo sui risultati effettivi?

Anche se contestati dai colleghi per le stime, la fondazione Rockwool, in collaborazione con James J. Heckman, ha rilevato che, in Danimarca, la differenza comparativa nella mobilità educativa generazionale è più o meno della stessa dimensione di quella degli Stati Uniti. Concludono gli studiosi:

Nonostante lo stato assistenziale molto più generoso della Danimarca, il suo ampio sistema di asili e le sue tasse scolastiche gratuite, la relazione influenza della famiglia/educazione del bambino è molto simile a quella degli Stati Uniti. In entrambi i paesi, buona parte dell'associazione media tra risorse parentali e livello di istruzione dei bambini può essere spiegato da fattori stabiliti entro i 15 anni, comprese le abilità dei bambini (Landersø e Heckman, 2016, p. 55).

I colleghi che mettono in discussione la conclusione di somiglianza tra Danimarca e Stati Uniti affermano che: «In Danimarca, il 20\% dei figli di genitori senza diploma di scuola superiore raggiunge un diploma universitario, mentre solo il 14\% dei bambini statunitensi lo raggiunge» (Andrade e Thomsen, 2018, p. 99). Può certamente accadere che il $6 \%$ in più di figli con 
genitori senza diploma di scuola superiore ottenga una laurea in Danimarca rispetto agli Stati Uniti, la domanda che trovo più importante è: che ne è dell' $80 \%$ che non lo raggiunge?

La conclusione generale è sempre la stessa: ai giovani provenienti da famiglie con un basso livello di istruzione non vengono offerte le stesse opportunità educative dei loro coetanei. In altre parole, l'uguaglianza generale in Danimarca non è affatto raggiunta attraverso l'offerta delle stesse opportunità educative, ma attraverso una redistribuzione del reddito. L'idea di opportunità formali inoltre maschera il fatto empirico della riproduzione delle disuguaglianze nell' educazione.

Questa riproduzione è documentata ormai da decenni (Hansen, 1995) e ancora il misconoscimento della natura di questa verità si confonde nel discorso pubblico e, in una certa misura, anche in quello scientifico. Un approccio molto promettente per smascherare tali misconoscimenti è il Capability Approach (Kjeldsen, 2014).

Perché i giovani provenienti da famiglie svantaggiate non colgano un'opportunità educativa apparentemente libera, sarà quindi la questione da affrontare.

In una prospettiva di capacità, coloro che diventano esclusi, o che scelgono di escludersi non cogliendo le opportunità educative offerte dal sistema di istruzione, diventano la cartina di tornasole per valutare fino a che punto una politica educativa promuova, nella pratica, una istruzione "giusta". L'approccio delle capacità ci permette dunque di dare una risposta alle domande sopra sollevate intorno al problema dell'uguaglianza nell'educazione. In base a questo approccio, una "capacità" non è solo una abilità acquisita. Il concetto di capability implica le opportunità formali e le risorse necessarie per praticarle. Per fare un esempio, non è sufficiente che gli alunni a scuola imparino a svolgere il loro ruolo di cittadini in termini di acquisizione di alti livelli di conoscenza civica e comprensione dei concetti relativi alla cittadinanza, se le strutture democratiche della società, in modi diversi, proibiscono all'individuo di diventare un cittadino coinvolto e partecipante. Le capabilities in questo senso sono anche chiamate libertà sostanziali e quindi strettamente correlate alla questione della possibilità effettiva di poter mettere in atto queste capacità o meno. Ad esempio, se l'individuo possa o meno cogliere un'offerta educativa. Un altro aspetto importante legato al concetto di capabilities è che le capacità sono valori, entro limiti ragionevoli, dal momento che «danno un ruolo centrale all'abilità effettiva di una persona di fare le diverse cose che desidera fare in quanto gli attribuisce valore» (Sen, 2009, p. 253). 
Le libertà sostanziali si differenziano da tutte le altre libertà di cui un individuo può godere, in quanto sono fondamentali per lo sviluppo umano dell'individuo stesso. Per questo motivo l'educazione è interpretata come un'importante libertà sostanziale, mentre in molte delle azioni della vita è un prerequisito per altre libertà sostanziali (ad esempio partecipare a un dibattito pubblico riguarda la capacità di ragionare come essere umano ecc.). L'educazione intesa come libertà sostanziale può quindi «fare riferimento alla capacità educativa come la vera libertà di scegliere un programma di formazione/curriculum che abbia una ragione di valore» (Bifulco, 2012, p. 1). Tuttavia, l'approccio delle capabilities è liberale, non neoliberista, e l'attenzione è posta sulle barriere, sulle opportunità e sulla scelta che l'individuo ha a portata di mano «piuttosto che sulla celebrazione di uno stile di vita particolare» (Sen, 2009, p. 238). L'approccio alle capabilities è liberale in un modo eticamente individualista, mentre il neo-liberismo, al contrario, è ontologicamente individualista (Walker, 2005, p. 106).

Le opportunità formative formali fornite in Danimarca possono quindi essere intese come mezzi per conseguire un'istruzione. Nell'ambito dell'approccio delle capacità, ciò che consente di passare dai mezzi per il conseguimento (materie prime e altri beni pubblici di cui l'individuo dispone) al raggiungimento effettivo vengono definiti fattori di conversione o circostanze contingenti. Sen indica cinque fonti di variazione che incidono sulla possibilità di trasformare beni, come l'istruzione gratuita, in risultati. Una di queste sono le caratteristiche personali legate a disabilità, malattia, età $o$ sesso. Allo stesso tempo, Sen mostra consapevolezza dei fattori di conversione relativi alla distribuzione all'interno della famiglia (Sen, 1999, p. 70).

Riassumendo: l'approccio basato sulle capacità rende visibile l'equilibrio tra opportunità formative formali (mezzi per conseguire), i fattori di conversione di questi mezzi in capacità (libertà di realizzazione) e risultati effettivi (conseguimento). Equilibrio in cui resta salva la scelta dell'individuo di trasformare - o non trasformare - queste opportunità in realtà.

\section{La competizione globale come motore dietro l'idea di educazione per tutti}

Poco prima della crisi economica, l'ex governo danese ha firmato un accordo politico su come reagire alla competizione economica globale (The Danish Goverment, 2006). L'obiettivo di questo accordo era quello di essere economicamente competitivi in futuro e i mezzi per raggiungere tale scopo 
si rivolgevano principalmente a questioni educative (Plant, 2006). Le preoccupazioni riguardavano la sicurezza delle risorse future per il welfare danese. Tra le soluzioni proposte c'era quella di aumentare la quota di educazione giovanile al $95 \%$.

L'obiettivo fissato stabiliva che il $95 \%$ di tutti i giovani dovesse raggiungere un livello di istruzione post-obbligatoria/secondaria superiore di tre anni. Il punto di vista dominante ritiene che un minimo di dodici anni di istruzione siano virtualmente un obbligo: «I giovani avranno il diritto e il dovere nei confronti dell'istruzione. Non appena i giovani contattano il comune per il sostentamento, il comune deve stipulare un accordo sulla formazione dei giovani» (Danish Government, 2013, p. 17 [propria traduzione]). Ho chiamato altrove questa tendenza verso l'istruzione per tutti "un carico di educazione permanente", mentre tutte le persone, giovani e anziane, ora sono diventate: «condannate allo sviluppo per tutta la vita» (Tulinius, 2012).

La domanda di istruzione per tutti a livello secondario superiore è però bifronte. Da un lato può essere sentita come un" "offerta che non si può rifiutare" e un onere permanente, dall'altro è possibile affermare che la sostanziale libertà di educazione è una delle cose più importanti per lo sviluppo di vite veramente umane (Nussbaum, 2010; Nussbaum, 2011). Si crea dunque una tensione tra, da un lato, le buone ragioni per rifiutare una particolare offerta educativa che non favorisce quella vita che l'individuo considera valida per sé, e dall'altro, l'importanza di investire per il proprio bene e per quello della società. Come dice Amartya Sen: «Per costruire un paese, occorre costruire una scuola» (Sen, 2002, p. 1). A ragione, la maggioranza dei cittadini delle società occidentali considera l'istruzione non solo come un diritto umano, ma anche come obbligo sociale di fornire opportunità educative per tutta la vita dell'individuo. In realtà si sostiene che: «La Danimarca ha una lunga tradizione nel migliorare le competenze della forza lavoro oltre le fasi obbligatorie dell'istruzione. In effetti, la nozione è che l'apprendimento è un'occupazione per tutta la vita» (Ministry of Education, 2010). La capacità di pensare in modo critico ed acquisire competenze di cittadinanza è conseguita attraverso l'istruzione di base, che è anche un luogo che contribuisce alla capacità di provare empatia per gli altri (Nussbaum, 2010, p. 7). I giovani nella scuola dell'obbligo in Danimarca, generalmente, non sembrano pensare che l'istruzione sia un dovere ingiusto e pesante da sopportare sulle spalle, al contrario, riferiscono in larga misura che si sentono inclusi dalla loro scuola, come dalla loro classe e dai loro insegnanti. Questo è uno dei principali risultati della valutazione nazionale condotta sul benessere nella scuola dell'obbligo (grado 0-9) che copre 506.784 alunni con un tasso di risposta del 92\% (Dansk Center for Undervisningsmiljø, 2016). Bisogna 
solo essere consapevoli che questa maggioranza possiede già le attitudini per l'educazione e lo sviluppo personale. Rimane un numero di alunni per cui le cose non stanno così, e l'alto benessere medio può essere per loro di poco conforto di fronte alle esperienze scolastiche negative che si aggiungono a un background familiare svantaggiato.

La presenza di esperienze scolastiche negative, tra quei giovani che abbandonano o non colgono le opportunità educative, non è solo una questione danese. Tale situazione è possibile rintracciarla in tutta l'Europa, dove i giovani svantaggiati «pensano che una delle minacce contro le aspirazioni per (ulteriori) apprendimenti, i desideri legati al lavoro e le idee di prospettive future sono le esperienze scolastiche negative (es. Danimarca, Germania, Svizzera, Francia)» (Kjeldsen, Ley, Jensen e Otto, 2012).

I giovani svantaggiati sperimentano il cambiamento discorsivo nelle politiche sociali ed educative in misura maggiore rispetto ai loro coetanei. Queste politiche alterate diventano un mezzo per la fine della ristrutturazione dello stato sociale nordico (Nordic welfare state) verso uno stato sociale competitivo (competitive social welfare state). Il principale fattore alla base di questo processo è noto come "la formazione generale dello stato competitivo". I paesi nordici, spesso caratterizzati come facenti parte di un particolare modello di welfare socialdemocratico, hanno sperimentato questo cambiamento con un ritmo elevato dall'inizio del XXI secolo. Un cambiamento che storicamente si può trovare dagli anni ' 80 come transizione dal welfare state al workfare state e adesso, nel nuovo millennio, verso un modello competitivo di "payfare" state. Questo ci porta alla prossima questione: come viene concettualizzato il gruppo di giovani che sceglie di non cogliere le offerte educative?

\section{NEET - ma quale bisogno?}

Sono gli studenti che abbandonano l'istruzione, o non colgono le opportunità educative, che metteremo a fuoco. "Un bambino amato ha molti nomi”, recita un tipico proverbio nordico. Questo vale anche per il gruppo sopra menzionato. Giovani che abbandonano la scuola, studenti svantaggiati, alunni con bisogni speciali ecc.

Diverse costruzioni concettuali cercano di definire questo gruppo e fornire misure statistiche comparative. Una di queste è il concetto di giovani non impegnati né nel lavoro, né nell'istruzione, né nella formazione (NEET), che a livello superficiale sembra semplice e definito, ma nella pratica statistica è complesso e ha molte applicazioni diverse (Elder, 2015). 
L'interesse per questa figura e le sue implicazioni si riscontra tra i responsabili politici e del settore pubblico in Danimarca, dove si sostiene che la «responsabilità riguardo al problema dei NEET e come affrontarlo ricada sul governo, che "suona il primo violino" insieme alle parti sociali in merito all'ALMP» (Jørgensen, 2012). Per rispondere ai bisogni dei NEET, le politiche e le statistiche ufficiali sono state considerate principalmente come parte delle Politiche Attive del Mercato del Lavoro (ALMP). Vale la pena notare che la questione del mancato inserimento in istruzione si traduce in ALMP che diventa problematica poiché smonta le ragioni e i risultati nelle due aree politiche dell' istruzione e del mercato del lavoro. Le differenze concettuali nell'applicazione della statistica NEET possono essere esplorate empiricamente. La figura 1 rappresenta il rapporto NEET diviso per età e sesso in Danimarca:

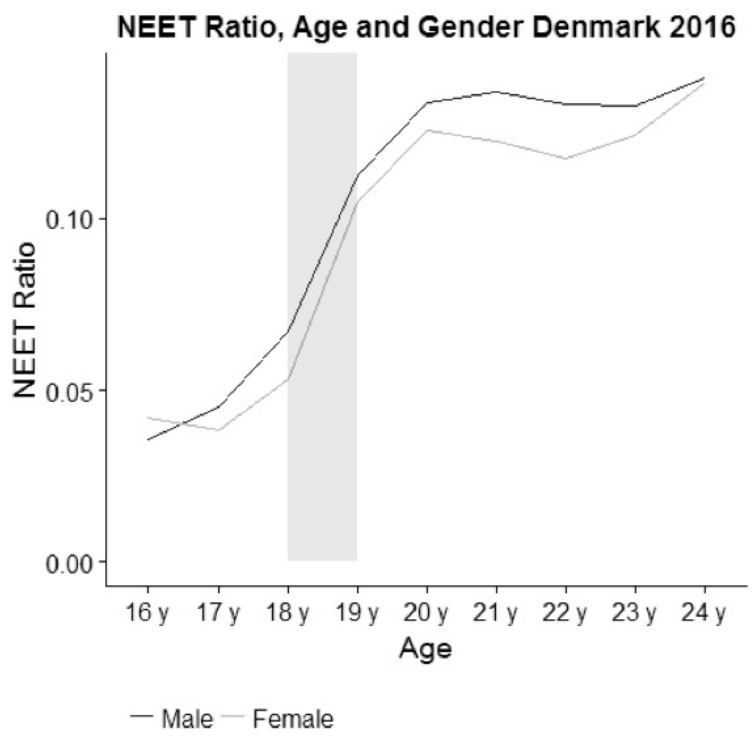

Fig. 1 - Distribuzione dei NEET per età e sesso in Danimarca

È abbastanza chiaro che tra i 18 e i 19 anni troviamo un notevole "salto" nella proporzione dei NEET della coorte completa. È quello il momento in cui i giovani, raggiungendo la maggiore età, diventano titolari di sussidi sociali e quindi un gruppo target per le politiche attive del mercato del lavoro (ALMP). Fino a quando i giovani non raggiungono la maggiore età e non necessitano di sussidi sociali, non vengono presi in considerazione e valutati con precisione dalle statistiche. 


\section{Assenza come dato non percepito di rifiuto delle oppor- tunità educative}

L'immagine del livello di inattività diventa piuttosto sfocata nelle statistiche NEET e fallisce non tenendo conto delle diverse forme di assenza di studenti formalmente iscritti a un'offerta formativa:

Un'altra difficoltà è dovuta alla mancanza di statistiche ufficiali sulla non-frequenza (dove la non-frequenza si riferisce agli studenti che non vanno a scuola o vi si recano di tanto in tanto, senza averlo ufficialmente comunicato alla scuola). Pertanto, abbiamo a che fare con un effetto che potremmo chiamare "ombrello" in quanto vi è una sovrapposizione (inappropriata) di abbandono (persone che hanno formalmente abbandonato la scuola senza iscriversi ad un'altra) e dispersione. Con effetto ombrello intendiamo che solo i numeri relativi all'abbandono formale sono usati per indicare la percentuale totale di dispersione. Infatti, anche se ci limitiamo a una definizione precisa di dispersione, il livello di studenti che frequentemente si allontanano da scuola sarà sempre molto più alto (Batini, Bartolucci e De Carlo, 2017).

\section{Assenza dalla scuola mentre si è ancora iscritti - una parte non riconosciuta del processo di riproduzione delle disuguaglianze nell'istruzione}

Per accedere al problema dei giovani che non hanno raggiunto l'obiettivo di portare al termine il percorso di istruzione secondaria superiore, nel 2017 è stato istituito un gruppo di esperti per fornire raccomandazioni ai responsabili delle politiche nazionali in Danimarca sui "modi migliori per raggiungere un'educazione della gioventù". Tra le loro raccomandazioni c'era un ulteriore miglioramento dell'ex obiettivo del $95 \%$ - che dovrebbe essere sostituito da un obiettivo del $100 \%$ che include quindi tutti i giovani. La maggior parte delle raccomandazioni fa riferimento a iniziative economiche per i comuni, mirate a favorire la transizione dall'istruzione al mercato del lavoro, alla diffusione geografica delle offerte e all'attuazione di una catena di responsabilità al fine di garantire che tutte le istituzioni emittenti siano tenute a gestire la transizione dei giovani da un'offerta all'altra.

Il gruppo di esperti ha rilevato che l'area educativa relativa ai giovani che non hanno né un'occupazione lavorativa né hanno conseguito un titolo di istruzione dopo 7 anni dall'uscita dall' istruzione obbligatoria, ha avuto non meno di 25 riforme politiche dal 2000. Gli esperti hanno inoltre focalizzato 
la propria attenzione sulla relazione esistente tra livello di assenze scolastiche e partecipanti a speciali corsi di istruzione secondaria (una seconda possibilità) che decidono poi di passare all'istruzione regolare. Il gruppo ha trovato una forte correlazione: «Più basso è l'assenteismo degli studenti, più è probabile che passeranno al normale percorso di istruzione dopo il corso speciale» (Expert group on better ways to a youth education, 2017, p. 43 [traduzione propria]). L'associazione tra la non frequenza scolastica nella scuola secondaria inferiore e la transizione all'istruzione secondaria superiore è stata contrassegnata solo come area di interesse. Essi concludono che: «[molte] alcune scuole già lavorano sistematicamente per prevenire le non frequenze, e la ricerca deve sostenere le scuole nell'adozione di misure che aiutano a mantenere gli alunni a scuola [...] strumenti devono essere dati e assegnati in modo inequivocabile alla gestione scolastica per combattere le non frequenze e per garantire che nessuno eviti l'insegnamento» (Expert group on better ways to a youth education, 2017, p. 72 [traduzione propria]).

\section{Dati e metodi}

L'istruzione obbligatoria e l'orientamento dei giovani nella transizione dall'istruzione secondaria inferiore all'istruzione secondaria superiore sono compiti a livello comunale. Durante la ricerca di uno dei programmi educativi per i giovani con bisogni educativi speciali, la cosiddetta educazione speciale organizzata per i giovani, si è dimostrato chiaramente che le possibilità e il contenuto effettivo dell' istruzione riguardano in larga misura le decisioni del comune (Andersen e Pedersen, 2008; Kjeldsen, 2014). In una certa misura, si potrebbe parlare di una "lotteria del codice postale" ${ }^{2}$ per quel che riguarda la traduzione di diritti formali in offerte reali. Bene in linea con la relazione che Bourdieu indica tra lo spazio sociale e lo spazio fisico. Bourdieu sostiene che: «non c'è spazio in una società gerarchizzata che non sia gerarchizzato» e prosegue affermando che: «in effetti, lo spazio sociale si traduce in spazio fisico, ma la traduzione è sempre più o meno sfocata» (Bourdieu, 1999, p. 124). La lotteria del codice postale può avere strutture più intrinseche di quelle che si credono guardando solo la superficie. Un'ulteriore importante scelta, quando si valuta la relazione tra spazio fisico e spazio sociale, riguarda l'unità di analisi e le statistiche disponibili, al fine di

\footnotetext{
${ }^{2}$ Ovvero, in questo caso, il fatto che la qualità dell'offerta educativa dipenda dalla via e dal quartiere in cui lo studente abita [N.d.C.].
} 
esplorare, cercando di comprendere, le forme che strutturano sia il sociale che lo spazio, creando «una mappa che si adatta al suo scopo e non distorce inutilmente i dati sottostanti» (Bivand, Pebesma e Gómes-Rubio, 2008, p. 1).

\subsection{Le scuole come unità di analisi e livello di indagine sociologica}

L'unità di analisi scelta è la scuola obbligatoria in Danimarca. La motivazione di questa scelta è legata a diversi fattori. In primo luogo, le scuole sono, come agenti istituzionali, sia un luogo fisico che sociale, dove possiamo trovare processi di inclusione ed esclusione. Naturalmente, le scuole hanno composizioni abbastanza diverse di alunni con un diverso background familiare in termini di status sociale, culturale ed economico. Una seconda motivazione è la disponibilità di diverse statistiche, che possono essere unite insieme. Il ministero dell'educazione offre, infatti, accesso a una serie di set di dati a livello aggregato. Questi possono essere suddivisi per ogni scuola con un numero ID individuale per istituto. Le scuole si trovano a un livello sociologico di analisi inteso, classicamente, come "meso". Ovviamente, registrare i dati con accesso a livello "micro", relativo ai singoli individui, estenderebbe le possibilità di queste analisi, ma in questo caso l'attenzione sarà concentrata sulla scuola come singolo dato contestuale. Si potrebbe inoltre sostenere che mentre il background familiare è un indicatore "statico", che è difficile da cambiare con la politica e la pratica professionale, le pratiche educative e didattiche all'interno delle scuole, e quindi il lavoro con questi giovani, possono essere cambiate, e ciò rafforza l'argomento a sostegno della scelta della scuola come unità di analisi principale.

Nell'ambito della sociologia spaziale è stato sostenuto che:

NONOSTANTE il diffuso interesse per la sociologia spaziale, la considerazione dello spazio nel campo della ricerca sulla stratificazione resta limitata. La letteratura ben documentata e coerente sulla stratificazione si trova principalmente su due scale spaziali opposte, la città e il livello transnazionale. Una grande letteratura urbana esplora le disuguaglianze di povertà, segregazione razziale, crimine e altre condizioni all'interno e tra le città. [...] Ma c'è un centro mancante: vaste aree di luoghi, persone e argomenti sostanziali non vengono sistematicamente studiati perché non riescono a cadere nel modello relativamente binario con cui i sociologi ritagliano lo spazio (Lobao, Hooks e Tickamyer, 2007, p. 29). 
Portare il livello istituzionale meso delle scuole e metterlo in relazione con le differenze comunali crea una rottura nel modello binario tra il livello macro nazionale e il livello micro di analisi.

I software di statistica commerciale (GIS, SAS, SPSS, STATA) offrono pacchetti per l'analisi spaziale. In questa analisi, invece, è stato utilizzato il software statistico R non commerciale (R Core Team, 2017) con una serie di pacchetti statistici (Bivand e Lewin-Koh, 2017; Kahle e Wickham, 2013; Bivand, Keitt e Rowlingson, 2017; Bivand, Ono e Dunlap, 2011). Per un'analisi spaziale di questo tipo è essenziale avere accesso a fonti autorevoli di divisione amministrativa in comuni, contee, giurisdizioni ecc., fondamentali per l'organizzazione della pubblica amministrazione e delle statistiche (KMS, 2012b). L'istituto National Survey and Cadastre in Danimarca ne è responsabile e fornisce mappe GIS (Geographic Information System). Pertanto è possibile correlare il luogo geografico delle scuole con i dati dell'autorità nelle mappe geografiche. Ai fini dell'analisi esplorativa, sono state applicate diverse variabili per l'unione dei diversi set di dati. Ad esempio, la variabile "KOMKODE" (codice del comune). Le mappe GIS e le unità amministrative fornite sono ad uso gratuito quando «si richiama l'attenzione sul copyright. [...] Con qualsiasi uso di temi, il copyright dell'Agenzia Catasto dovrebbe essere dichiarato» (KMS, 2012a). La mappa in questo capitolo si basa sui file di forma GIS dell'agenzia Catasto.

\section{Risultati}

Diamo prima un'occhiata alla mappa cartografica della Danimarca (fig. 4). Lo strato di base della mappa è composto da 98 comuni danesi. Rappresenta i diversi livelli comunali dei giovani che hanno lasciato l'istruzione obbligatoria danese $-\mathrm{o}$ avendo passato un esame finale o lasciando la scuola prima - e che non risultano iscritti a nessun insegnamento riconosciuto tre mesi dopo. Una parte dei giovani è possibile che sia inserita nel mercato del lavoro, anche se è più probabile che questi siano sostenuti dalla famiglia, in cosiderazione della loro età, e per questo motivo non siano considerati parte della forza lavoro. Visto da una prospettiva educativa con un obiettivo del $95 \%$, questi giovani sono ancora "non identificati" ${ }^{3}$ in relazione alla loro istruzione. Lo strato superiore della mappa è la collocazione geografica delle

${ }^{3}$ Adattamento italiano del termine "unknowns", utilizzato dall'autore: studenti che non rientrano nei conteggi ufficiali [N.d.C.]. 
scuole obbligatorie. Sono illustrati con punti di dimensioni che rappresentano il numero di alunni iscritti tra 7 e 10. Infine, i punti delle scuole sono colorati proporzionalmente alla proporzione di studenti che hanno oltre il $10 \%$ delle assenze "illegali" (non frequenze) da scuola. Il tasso di assenza scolastica e le diverse categorie richiedono ulteriori spiegazioni.

\subsection{Le non frequenze in una prospettiva legislativa}

Al fine di monitorare il tasso complessivo di assenze scolastiche degli alunni, la legislazione nazionale impone che il dirigente scolastico e gli insegnanti debbano registrare quotidianamente la frequenza durante le lezioni. La motivazione relativa all'assenza degli alunni viene quindi registrata elettronicamente nei sistemi di amministrazione degli studenti in tre categorie distinte: 1) assenza dovuta alla malattia dello studente, 2) assenza con il permesso del dirigente scolastico, 3) assenza illegale. Questa informazione sull'assenza è inoltre accessibile al consiglio comunale (Ministry of Education, 2014). La distribuzione di queste tre categorie tra le scuole viene evidenziata nel seguente boxplot:

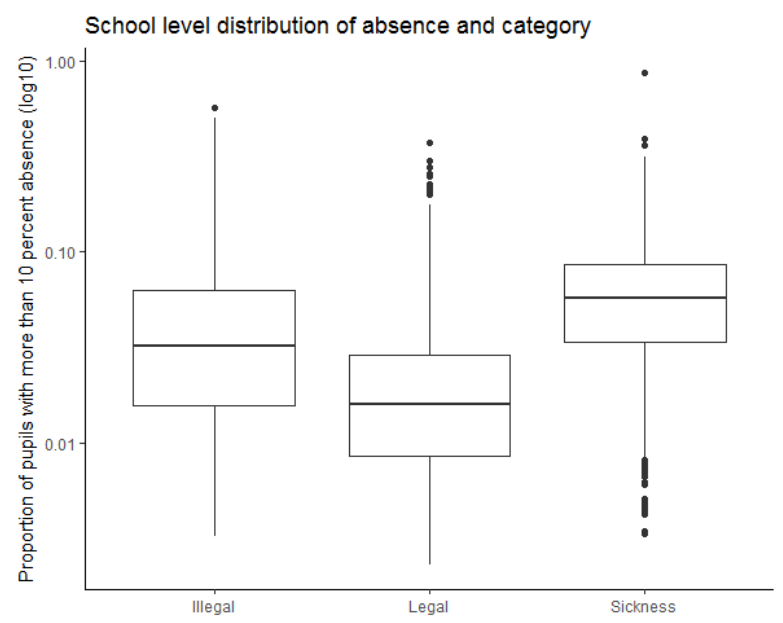

Fig. 2 - Distribuzione delle tre tipologie di assenze

La maggior parte delle scuole ha livelli molto bassi di tutte e tre le categorie e quindi il boxplot è stato ridimensionato su scala logaritmica.

Tra gli alunni con una percentuale di assenze superiori al 10\%, la tipologia di assenza meno diffusa è quella per malattia, mentre la più diffusa è 
l'assenza illegale. L'assenza legale, infine, ha anch'essa una diffusione piuttosto è bassa.

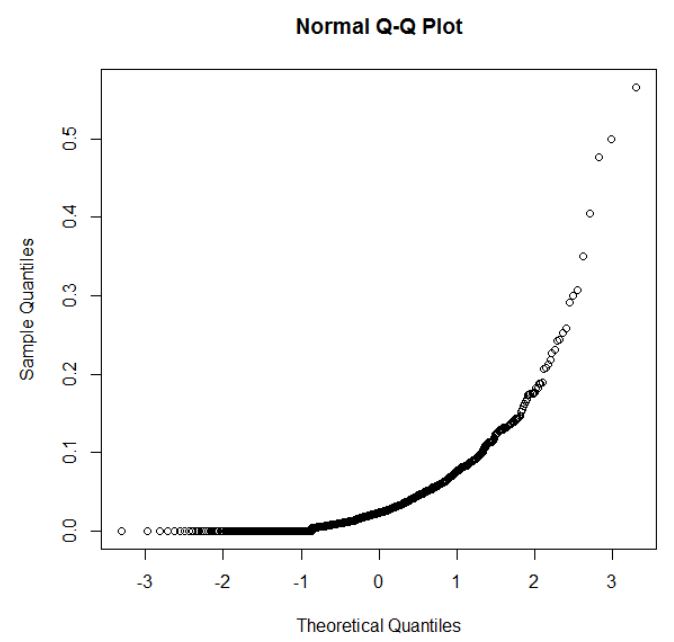

Fig. 3 - Rappresentazione dei quantili della distribuzione delle assenze

Il livello di assenza scolastica illegale tra le scuole non segue una distribuzione normale, come si può ricavare dal grafico QQ (fig. 3). Infatti, per il primo decimo percentile di scuole non viene rilevata un'assenza scolastica illegale tra i propri alunni, mentre le scuole superiori al $90^{\circ}$ percentile hanno non meno del 9,6\% degli alunni che hanno oltre il 10\% di assenza illegale da scuola.

In una prospettiva legislativa in caso di assenza illegale, il preside della scuola dovrebbe contattare immediatamente i genitori dell'alunno per identificare la causa dell'assenza. Inoltre, il dirigente scolastico dovrebbe cercare di risolvere i problemi in collaborazione con lo studente e i genitori, eventualmente con il coinvolgimento di una consulenza psicologica pedagogica, la "Guida Educativa per i Giovani", il servizio sanitario municipale o altre istituzioni. Se non è possibile stabilire il dialogo necessario con lo studente e i genitori, il dirigente della scuola dovrebbe coinvolgere il consiglio comunale. Queste precauzioni dovrebbero essere attuate quando si registra che uno studente è assente illegalmente da una settimana, anche nel caso in cui si tratti della prima settimana dopo la fine del congedo estivo. L'assenza illegale è inoltre legata all'erogazione degli assegni familiari che la famiglia, eventualmente, riceve. In caso di assenza illegale a lungo termine, il comune 
può infatti rivedere al dettaglio il pagamento relativo al mantenimento dei figli.

\subsection{Elevati livelli di assenze illegali raggruppate all'interno delle città più grandi}

La fig. 4 mostra i raggruppamenti dei NEET nelle diverse municipalità danesi. Le scuole con alti livelli di assenze illegali sembrano raggrupparsi nell'area delle cinque maggiori città della Danimarca. Sono in ordine decrescente: Copenaghen, Aarhus, Odense, Aalborg ed Esbjerg. Il livello di "incognite" è distribuito in relazione alle città più grandi, ma è possibile trovare altre influenze.

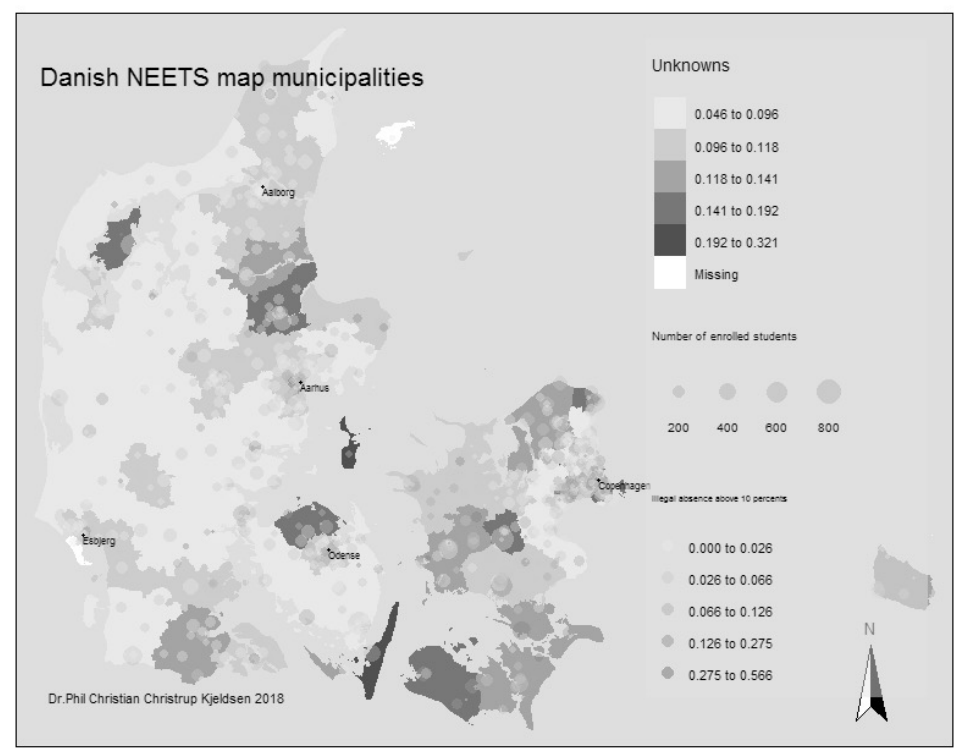

Fig. 4 - Distribuzione dei NEET nelle municipalità danesi

Mette Pless e Noemi Katznelson hanno studiato la condizione degli alunni usciti dalla scuola dell'obbligo dopo il $9^{\circ}$ grado, rilevando la loro situazione un anno dopo. Le studiose hanno trovato una connessione tra la zona di residenza degli studenti e la loro scelta educativa. Nella campagna, i giovani preferiscono scegliere di frequentare la scuola secondaria inferiore di I grado o trasferirsi in un collegio (in un contesto danese il collegio è 
spesso un anno di scuola con diverse materie extrascolastiche come teatro, sport o musica) mentre nei giovani provenienti dalle grandi città è più probabile che venga scelta un'istruzione secondaria superiore accademica. Inoltre hanno scoperto che il 13\% degli alunni considerava difficile il passaggio dalla scuola dell'obbligo ad altre offerte formative (Pless e Katznelson, 2006).

Sempre seguendo la mappa, i problemi con la transizione sembrano avere due modalità. Una connessa alle città più grandi e un'altra connessa alla campagna. Quest'ultima dimensione può essere in certa misura spiegata dall'accessibilità geografica delle offerte per l'istruzione secondaria superiore professionale. Si sostiene che i contesti strutturali e il contenuto dell'istruzione professionale non promuovono la scelta dell'istruzione secondaria professionale degli studenti, mentre una distribuzione geografica distorta dell'istruzione può farlo (Local Government Denmark (KL), 2017a; 2017b). L'altra dimensione potrebbe piuttosto essere legata all'associazione tra l'assenza illegale e il non accedere alle diverse opportunità educative.

\subsection{Associazioni tra assenze illegali e mancata iscrizione al livello scolastico successivo}

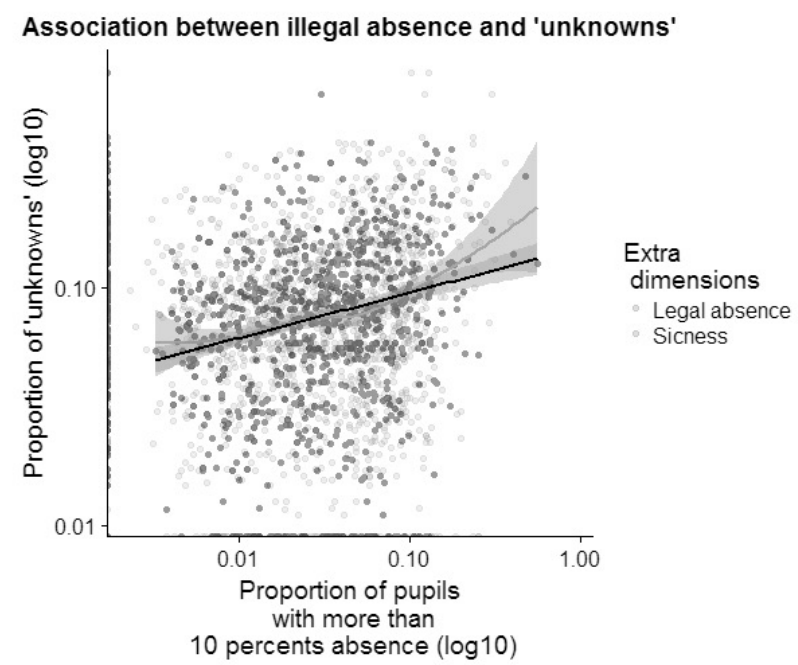

Fig. 5 - Associazione tra assenze illegali e studenti "non identificati"

Se si guarda alla correlazione momento-prodotto di Pearson tra la percentuale di alunni dal $7^{\circ}$ al $10^{\circ}$ grado in ogni scuola con oltre il $10 \%$ di assenza 
scolastica illegale e si valuta l'associazione alla proporzione di studenti (non necessariamente gli stessi studenti) di questa scuola tre mesi dopo aver lasciato la scuola, troviamo un'associazione statisticamente significativa ( $\mathrm{p}$ value $=2,974 \mathrm{e}-11$ ). Anche se il coefficiente di correlazione rivela un'associazione debole ( cor $=0,21$ : all'interno dell'intervallo di confidenza al 95\% 0,15 e 0,27 ), esiste comunque un'associazione. Tenendo presente che questa relazione è a livello scolastico e non si trova nelle statistiche a coppie dei microdati, rimane comunque un livello di correlazione che vale la pena di notare. Questo dato supporta l'interpretazione che le assenze scolastiche illegali e in seguito il mancato proseguimento scolastico sono due facce della stessa medaglia. Dalla ricerca qualitativa, troviamo esempi dell'arbitrarietà nell'orientamento per questa tipologia di soggetti. Spesso perdono la loro strada e le loro aspirazioni mentre sono ancora nella scuola dell'obbligo e queste esperienze scolastiche negative li seguiranno e segneranno per tutta la vita. Questo gioca un ruolo nella riproduzione delle disuguaglianze sociali. In base a dati relativi al 2016/2017, più di un quarto degli alunni (27\%), di ogni grado, i cui genitori hanno un grado di istruzione basso, ha più del $10 \%$ di assenze scolastiche. Un dato drammaticamente superiore rispetto agli alunni provenienti da famiglie con genitori con un'istruzione terziaria, dove il rapporto scende a meno di un decimo degli studenti (9\%). Bisogna anche essere consapevoli che l'assenza si riferisce ai giorni effettivi in cui l'alunno avrebbe potuto frequentare l'insegnamento. Durante lo sviluppo storico del sistema scolastico obbligatorio danese, il numero di "settimane scolastiche" e di giorni è passato a 40 settimane, coprendo 200 giorni di scolarizzazione. Tenendo presente questo, il $27 \%$ di tutti gli alunni i cui genitori hanno la scuola elementare come livello di istruzione più alto è assente almeno 20 giorni durante l'anno scolastico. Per un alunno medio, in questa categoria, si calcola una perdita di tre quarti di anno scolastico nel periodo dell'istruzione obbligatoria.

Di seguito analizzeremo, in breve, l'associazione tra il clima scolastico e il livello di non frequenze illegali. La valutazione del benessere, condotta ogni anno a livello nazionale, ha 40 domande e i risultati sono calcolati a livelli scolastici aggregati dal $7^{\circ}$ al $10^{\circ}$ grado. Anche se il tasso di risposta è molto alto, c'è ancora una considerevole probabilità che per gli studenti con alto grado di non frequenze il numero di rispondenti sia sottostimato (non hanno partecipato al test mentre erano assenti), quindi i correlati potrebbero essere più alti nelle seguenti domande: "Sei stato vittima di bullismo in questo anno scolastico?" (fig. 6) e "Gli altri studenti mi accettano per come sono?" (fig. 7). 


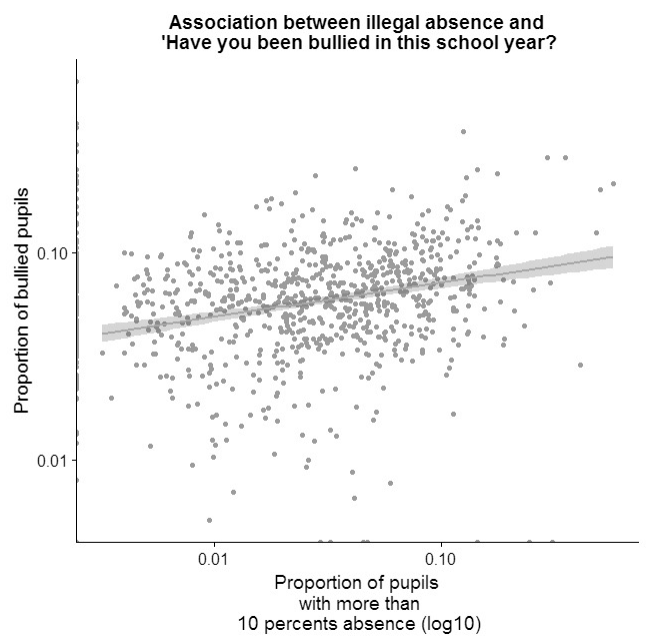

Fig. 6 - Associazione tra non frequenze ed essere stati vittime di bullismo

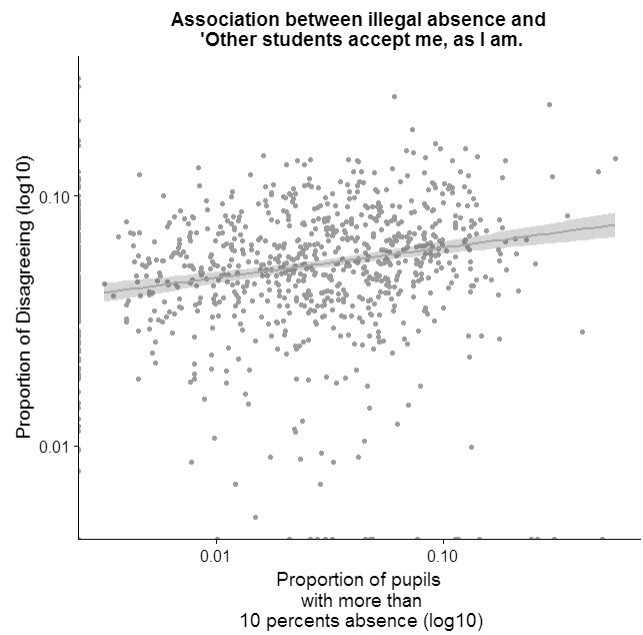

Fig. 7 - Associazione tra non frequenze e percezione di non accettazione da parte degli altri studenti

Per la domanda "Gli altri studenti mi accettano per come sono", una relazione statisticamente molto debole con la non frequenza illegale superiore al $10 \%$, può essere trovata nel test per la correlazione del momento-prodotto di Pearson ( $p$-value $=5,993 \mathrm{e}-07$; cor $=0,16$ ). L'associazione è statisticamente più significativa e più forte per la domanda essere vittima di bullismo ( $\mathrm{p}$-value $=9,181 \mathrm{e}-15$; cor $=0,25)$. Quando andiamo a calcolare la correlazione tra i livelli di accettazione fra studenti e la proporzione dei cosiddetti 
"non identificati", tre mesi dopo aver lasciato la scuola, non esiste una relazione significativa. Per quanto riguarda, invece, la relazione tra alunni che riferiscono di essere stati vittime di bullismo e la percentuale di studenti che non si iscrivono a un ulteriore grado di istruzione si può trovare una relazione statisticamente significativa $(\mathrm{p}$-value $=3,516 \mathrm{e}-12$; cor $=0,23)$.

Visto da una prospettiva di capabilities, ciò è da interpretare come fattore di conversione sociale ingiusto. La frequenza o la non frequenza illegale non possono essere spiegate da una scelta ragionata individuale sulla base di ciò che lo studente apprezza, ma piuttosto da una situazione in cui le scuole non sono funzionali nel promuovere e formare relazioni positive. La capacità ( $\mathrm{ca}$ pabilities) di istruzione sarà raggiunta solo se l'individuo si trova in una situazione in cui le capacità centrali proposte da Nussbaum: "ragionamento pratico", "integrità fisica" e "affiliazione" sono raggiunti.

Il ragionamento pratico indica la capacità di formare una percezione del bene e impegnarsi criticamente nella propria vita e all'interno di un contesto che consente l'attualizzazione di questa capacità (Nussbaum, 2011, pp. 24, 34). La base informativa per condurre un ragionamento pratico nella scelta dell'istruzione superiore è probabilmente strutturalmente correlata al capitale culturale dei genitori (Bourdieu, 1986). Si è riscontrato che il 14\% dei giovani il cui livello di istruzione della madre è la scuola elementare, risponde che non parla molto di istruzione e lavoro. Nel caso di giovani la cui madre ha un livello di istruzione più alto, questo dato scende all' $8 \%$ (Danmarks Evalueringsinstitut, 2017). Il senso di sicurezza in relazione ad alcune forme di bullismo assume il significato di «essere al sicuro da assalti violenti» (Nussbaum, 2011, p. 33) e il possedere capacità di affiliazione è allo stesso modo legato al bullismo e all'accettazione come un individuo uguale avendo la sicurezza di possedere «basi sociali di rispetto di sé e non umiliazione; essere in grado di essere trattato come un essere dignitoso il cui valore è uguale a quello degli altri». Ciò comporta disposizioni di non discriminazione in base a razza, sesso, orientamento sessuale, etnia, casta, religione, origine nazionale» (Nussbaum, 2011).

L'istruzione è quasi l'unico biglietto valido per l'ingresso nel mercato del lavoro dopo la ristrutturazione dello stato sociale danese in uno stato di payfare competitivo (Jensen e Kjeldsen, 2012). Il mancato completamento del percorso di istruzione si traduce pertanto in disuguaglianze economiche. Questa forma di disuguaglianza riprodotta tra generazioni cresce in Danimarca, dove un terzo dei bambini provenienti dalla classe svantaggiata finisce in questa situazione da adulti. Questo dato era "solo" un quarto dieci anni fa (Juul e Blicher, 2017). 


\section{Conclusioni}

Gli alunni svantaggiati nella scuola dell'obbligo potrebbero presentare la stessa domanda del testo della famosa canzone dei Clash: Should I stay or should I go? ("Dovrei restare o dovrei andare?").

Questa analisi punta, come altri risultati qualitativi precedenti (Otto, 2015), alla conclusione che il gruppo di alunni che abbandonano, o non acquisiscono ulteriori opportunità di istruzione dopo la scuola dell'obbligo, hanno spesso sperimentato di essere esposti a esperienze scolastiche negative. La risposta alla domanda può quindi spesso diventare un "sì, è meglio che tu vada via", il che solleva la problematica delle assenze illegali che nel corso della scolarizzazione si traduce in risultati accademici inferiori.

Sempre il testo dei Clash dice: If I go, there will be trouble. And if I stay it will be double ("Se vado, ci saranno problemi. E se rimango saranno il doppio") (The Clash, 1982). Solo sistemi educativi giusti potrebbero favorire pari opportunità non solo formali. La giustizia sociale nell'educazione promuove pratiche che forniscono all'individuo le libertà sostanziali centrali. Libertà dall'essere esposti al bullismo, ad esempio. Il problema è che è l'individuo che reagisce all'ambiente didattico e culturale della scuola "andando via" che paga il prezzo, abbastanza concreto in quote di reddito vitale, e che sperimenta i "problemi" che una vita senza un livello sufficiente di istruzione per competere in un mercato del lavoro altamente competitivo, fornisce.

Invece di concentrarsi sul singolo dropout, si dovrebbe focalizzare l'attenzione sull'ambiente scolastico e sulla didattica. Non è solo una questione di assenza o preferenze scolastiche. Il livello accademico dei giovani, acquisito attraverso l'istruzione scolastica, gioca un ruolo importante. È possibile trovare un'alta correlazione tra l'educazione dei genitori e i primi risultati dei test nella valutazione nazionale. Anche nei primi anni di scuola gli alunni delle famiglie con un alto livello di istruzione e provenienti dalla media o alta borghesia, hanno risultati migliori nei test nazionali (Pihl e Salmon, 2017), sembra ancora che ciò possa essere spiegato dalle assenze.

È difficile imparare qualcosa dalla scuola se non si partecipa.

\section{Riferimenti bibliografici}

Andersen J. and Pedersen A.H. (2008), "Ungdomsuddannelse for unge med særlige behov Unge i gang med uddannelsen i perioden 1. august 2007 - 31. januar 2008”, UNI-C Statistic og Analyse, Copenhagen.

Andrade S.B. and Thomsen, J.P. (2018), "Intergenerational Educational Mobility in Denmark and the United States", Sociological Science, 93-113. 
Batini F., Bartolucci M. and Carlo E.D. (2017), "Fight Dispersion Through Education: The Results of the First Cycle of the NoOut Project", Mind, Brain, and Education, XI, 4: 201212.

Bifulco L. (2012), "Introduction to the case studies", Social Work and Society, 10, 1.

Bivand R.S., Pebesma E.J. and Gómes-Rubio V. (2008), Applied Spatial Data Analysis with $R$, Springer Science + Business Media, New York.

Bivand R. and Lewin-Koh N. (2017), maptools: Tools for Reading and Handling Spatial Objects.

Bivand R., Keitt T. and Rowlingson B. (2017), rgdal: Bindings for the 'Geospatial' Data Abstraction Library.

Bivand R., Ono H. and Dunlap R. (2011), classInt: Choose univariate class intervals. Manual: R package version 0.1-17, testo disponibile al sito: http://CRAN.R-project.org/package $=$ classInt.

Bourdieu P. (1986), The Forms of Capital, in Richardson J., Handbook of Theory and Research for the Sociology of Education, pp. 241-258, Greenwood, New York.

Bourdieu P. (1999), Site Effects, in Bourdieu P. et al., The Weight of the World: Social Suffering in Contemporary Society, pp. 123-129, Blackwell Publishers Ltd, Oxford.

Bourdieu P. (2007 [1979]), Distinction: A social critique of the judgement of taste, Routledge, London.

Bourdieu P. and Passeron J.C. (1990), Reproduction in Education, Society and Culture, Sage Publications, London.

Danish Government (2006), Fremgang, fornyelse og tryghed strategi for danmark $i$ den globale, Schultz Information, Albertslund.

Danish Government (2013), Veekstplan DK: Stcerke virksomheder, flere job (Growth Plan DK: strong companies, more jobs), Copenhagen.

Danmarks Evalueringsinstitut (2017), Uddannelsesvalg $i$ 8. klasse: Unges vej mod ungdomsuddannelse, EVA, Copenhagen.

Dansk Center for Undervisningsmiljø (2016), Resultater fra den nationale trivselsmåling 2016: - tabelrapport, DCUM, Copenhagen.

Elder S. (2015), "What does NEETs mean and why is the concept so easily misinterpreted?", Technical Brief No.1, International Labour Office (ILO), Genève.

Expert group on better ways to a youth education (2017), Bedre veje til en ungdomsuddannelse: - anbefalinger til regeringen, The Ministry of Education, Copenhagen.

Hansen E.J. (1995), En generation blev voksen, Social Forsknings Instituttet, Copenhagen.

Jørgensen C. (2012), Denmark: ERM comparable analytical report on young people Not in Employment, Education and Training (NEETs), European Foundation for the Improvement of Living and Working Conditions.

Jensen N.R. and Kjeldsen C.C. (2012), "Pedagogy Back on Track; Enhancing Capabilities for Young People in Education and Work", Social Work \& Society, 10, 1.

Juul J.S. and Blicher S.P. (2017), Underklassens børn havner oftere selv i underklassen som voksen, Arbejderbevægelsens Erhvervsråd, Copenhagen.

Kahle D. and Wickham H. (2013), "ggmap: Spatial Visualization with ggplot2", The R Journal, 5,1: 144-161.

Kjeldsen C.C. (2014), Capabilities and Special Needs: An Educational Foundation, Verlag Dr. Kovac, Hamburg.

Kjeldsen C.C. (2016), Hvad er den pcedagogiske sociologi, in Dorf H. and Jensen N.R., Studier i paedagogisk sociologi, pp. 19-29, Aarhus Universitetsforlag, Copenhagen.

Kjeldsen C.C., Ley T., Jensen N.R. and Otto H.U. (2012), “A Comparative Comment on the Case Studies", Social Work \& Society, 10,1. 
KMS [The National Survey and Cadastre] (2012a, April 15), Rammer for DAGI: Kort og Matrikelstyrelsen, testo disponibile al sito: http://www.kms.dk/Produktkata$\log /$ DAGI/Rammer_for_DAGI.htm.

KMS (2012b, April 15), DA GI - Danmarks Administrative Geografiske Inddeling, testo disponibile al sito: http://www.kms.dk/Produktkatalog/DAGI/.

Landersø R. and Heckman J.J. (2016), The Scandinavian Fantasy: The Sources of Intergenerational Mobility in Denmark and the U.S., The Rockwool Foundation, Copenhagen.

Lobao L.M., Hooks G. and Tickamyer A.R. (2007), The Sociology of Spatial Inequality, State University of New York Press, Albany.

Local Government Denmark (KL) (2017a), Godt på vej-Alle unge skal med, KL, Copenhagen.

Local Government Denmark (KL) (2017b), Godt på vej: Mod uddannelse og job, KL, Copenhagen.

Ministry of Education (LBK no. 671 of 21/06/2010), Bekendtgørelse af lov om vejledning om uddannelse og erhverv samt pligt til uddannelse, beskaftigelse m.v., Retsinformation, Copenhagen.

Ministry of Education (2014), BEK nr 696 af 23/06/2014 Bekendtgørelse om elevers fraver fra undervisningen i folkeskolen, Retsinfo, Copenhagen.

Nussbaum M. (2010), Not For Profit: Why Democracy Needs the Humanities, Princeton University Press, New Jersey.

Nussbaum M. (2011), Creating Capabilities: The human Development Approach, The Belknap Press of Harvard University Press, London.

Otto H.U. (2015), Facing Trajectories from School to Work: Towards a Capability-Friendly Youth Policy in Europe. Springer International Publishing.

Pihl M.D. and Salmon R. (2017), Social arv i nationale tests: Tidlig indsats er vejen til at bryde den negative sociale arv, Arbejderbevægelsens Erhvervsråd, Copenhagen.

Plant P. (2006, September), Globalisering: 333 forslag - mange med en vejledningskomponent, testo disponibile al sito: http:/www.ug.dk/flereomraader/videnscenter/vejltemaer/viavejledning/globalisering_333_forslag_-_mange_med_en_vejledningskomponent peter plant.aspx.

Pless M. and Katznelson N. (2006), Unge ét år efter niende klasse: - anden delrapport om unges uddannelsesvalg og overgang fra grundskole til ungdomsuddannelse og arbejde. KVANTITATIVE RESULTATER, Center for Ungdomsforskning, Copenhagen.

R Core Team (2017), R: A Language and Environment for Statistical Computing, CRAN, Vienna, Austria.

Rodgers M. (2014), Økonom: Adgang til uddannelse for alle er nøglen til lighed, Information. Sen A. (1999), Development as Freedom, Oxford University Press, Oxford.

Sen A. (2009), The Idea of Justice, Penguin Books, London.

Sen A. (2002), "To Build A Country, Build A Schoolhouse”, New York Times, 27.

The Clash (1982), "Should I stay or should I go", Album: Combat Rock, Epic Records.

Tulinius B. (2012), "Den nye alderdom: Dømt til livslang udvikling”, AU Gustus, 2: 16-20.

Walker M. (2005), “Amartya Sen's Capability Approach and Education”, Educational Action Research, 13, 1: 103-110. 


\section{Appendice}

\section{Organizzazione del sistema scolastico danese ${ }^{1}$}

Il sistema scolastico danese prevede un'educazione prescolare non obbligatoria, un'istruzione di base obbligatoria e un'istruzione secondaria superiore (non obbligatoria).

\section{EDUCAZIOME PRESCOLARE}

Le scuole preprimarie sono di tre tipi:

- $\quad$ asili nido (Vuggestue - dai 6 mesi ai 3 anni);

- scuole materne (Børnehave - dai 3 ai 5/6 anni);

- istituti primari comprensivi (Aldersintegrerede institutioner - da un anno ai $5 / 6$ anni).

Questo livello non fa parte del sistema educativo (fatta eccezione per la Børnehaveklasse che rientra nel livello prescolare ma è parte integrale del ciclo obbligatorio). Questi istituti sono finanziati dalle autorità locali, i genitori pagano una retta mensile in base al reddito della famiglia.

\section{ISTRUZIONE OBBLIGATORIA}

L'istruzione obbligatoria ha la durata di 10 anni e comprende un anno di istruzione preprimaria (Børnehaveklasse: classe prescolare, 6-7 anni) e 9 anni di istruzione di base (Folkeskole: 7-16/17 anni) che corrisponde, articolandosi in un'unica struttura, al livello primario e secondario inferiore.

La maggior parte delle Folkeskole offre, tuttavia, anche un undicesimo anno facoltativo.

L'obbligatorietà è dell'istruzione e non della scolarizzazione di per sé, per cui, non è necessario che l'istruzione sia impartita nelle scuole pubbliche ma può anche essere offerta da scuole private o a casa (a condizione che sia comparabile con ciò che viene richiesto nelle scuole pubbliche).

L'istruzione nella Folkeskole è gratuita.

Nelle Folkeskole sono presenti insegnanti specialisti per materia e un insegnante di classe (generalmente l'insegnante di danese) che pianifica e organizza l'insegnamento in collaborazione con i colleghi che seguono la stessa classe.

Nella Folkeskole si applica la valutazione continua e il passaggio alla classe successiva è automatico. Il progresso generale dell'alunno (non i voti) in ogni materia viene comunicato almeno due volte all'anno ai genitori fino al settimo anno. Dall'ottavo anno, viene dato un voto nelle discipline in cui può essere sostenuto l'esame finale. Tutti gli studenti ricevono un certificato finale che riporta le discipline studiate, i voti ottenuti e i risultati dell'esame finale.

A cura di Irene D.M. Scierri e Marco Bartolucci. Per ulteriori approfondimenti si veda: http://www.indire.it/lucabas/lkmw_file/eurydice/BOLLETTINO_sistemi_scolastici_UE_2012_finale_x_web.pdf 


\section{ISTRUZIONE SECONDARIA SUPERIORE}

L'istruzione secondaria superiore (non obbligatoria) ha una durata di $2 / 3$ anni, ed è articolata in una istruzione generale, pensata prevalentemente per l'accesso all'istruzione universitaria:

- Gymnasium - STX (16-19 anni): percorso triennale di tipo accademico, prepara per gli studi a livello universitario o equivalente

- Højere Forberedelseseksamen - HF (17-19 anni): percorso biennale di tipo accademico, a cui si accede dopo l'ultimo anno facoltativo della Folkeskole (16-17 anni), prepara gli studenti ad affrontare gli esami a livello superiore in una o più discipline

- Højere handlsehsamen e Højere teknisk eksamen - HTX (16-19 anni): percorsi triennali che combinano un'istruzione commerciale (Højere handlsehsamen) o tecnica (Højere teknisk eksamen) e un'istruzione generale

e in una istruzione e formazione professionale (EUX) (16-19 anni), pensata prevalentemente per l'accesso al mercato del lavoro.

Tutti i programmi includono una fase introduttiva comune, con discipline dell'area umanistica e dell'area delle scienze sociali e naturali. Successivamente gli studenti scelgono un indirizzo più specifico. L'istruzione e la formazione professionale combinano lo studio di discipline professionali e generali a scuola in alternanza a periodi di stage in impresa.

Tutti i percorsi prevedono un esame finale che permette di accedere all'istruzione universitaria (o equivalente) e non hanno tasse di iscrizione.

La progressione da un anno all'altro è automatica a patto che siano state seguite tutte le lezioni e siano stati superati gli esami. Gli studenti possono anche ripetere un anno, nel caso sia ritenuto necessario.

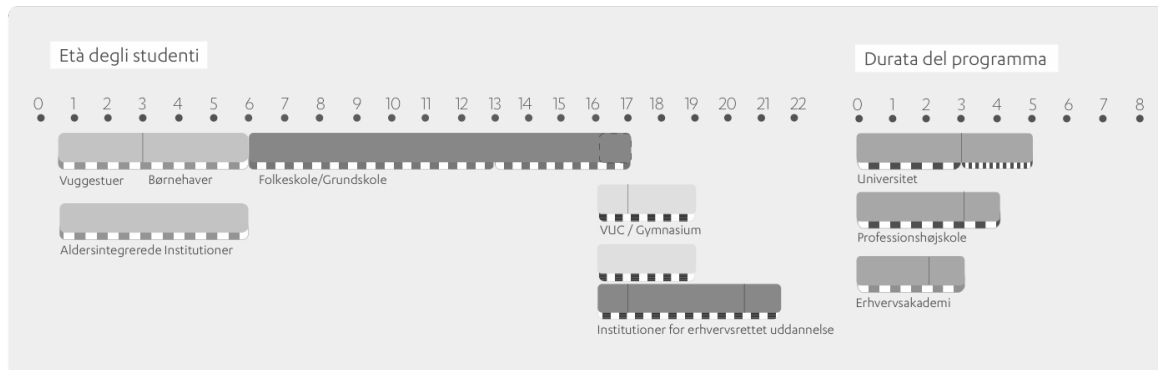

Fig. 1 - Diagramma del sistema educativo danese (Fonte: Strutture dei sistemi educativi europei: diagrammi 2017/2018, Eurydice Italia, 2017) 


\section{La dispersione scolastica e le politiche territoriali di welfare: prevenzione, contrasto e azioni di recupero. Dal piano europeo e nazionale alla realtà umbra}

di Rosario Salvato

\section{II ruolo del territorio nel contrasto alla dispersione}

\subsection{Piano triennale del diritto allo studio 2013-2015}

La riflessione proposta in questo contributo tende a mettere in evidenza l'ampio dispositivo legislativo e normativo, europeo, nazionale e regionale, capace di dare attuazione agli interventi di contrasto alla dispersione nelle realtà territoriali. Rimane assolutamente aperta, e trattata ampiamente nelle altre parti del volume, la netta differenziazione lessicale, formale e sostanziale nella descrizione del fenomeno, relativamente agli abbandoni formalizzati e senza formalizzazione. Ovviamente, nuove forme di dispersione scolastica incombono: le percentuali relative agli abbandoni nel contesto degli immigrati di seconda generazione appaiono drammatiche e dovranno essere tema di ulteriore ricerca, con interventi mirati di grande urgenza (Azzolini e Barone, 2012; Archambault et al., 2017).

Tra gli strumenti più recenti ed efficaci dal punto di vista operativo, il documento del Piano triennale per il diritto allo studio fornisce indirizzo alle politiche educative, formative e del lavoro, definendo obiettivi, priorità $\mathrm{e}$ strategie inerenti il sistema scolastico regionale. Il piano si muove in coerenza con il livello nazionale ed europeo, rispettando e perseguendo gli obiettivi della strategia Europa 2020. Tale documento costituisce un elemento guida per «le politiche dell'apprendimento, intese come l'insieme delle azioni rivolte a sviluppare l'innovazione, qualificare e sostenere il mercato del lavoro, supportare l'inclusione sociale e la cittadinanza attiva attraverso la qualificazione del capitale umano lungo il corso della vita» (Regione Umbria, 2013 p. 31). 
Con riferimento alla Raccomandazione del Consiglio Europeo del 28 giugno 2011 sulle politiche di riduzione dell'abbandono scolastico, nella Regione Umbria viene garantito il diritto all'apprendimento e all'istruzione mediante interventi mirati per il sostegno della frequenza scolastica, per la riduzione dell'insuccesso, nonché per il contrasto dell'abbandono scolastico.

In riferimento al problema dell'abbandono degli studi, il Piano propone precisi obiettivi che puntano ad una riduzione del fallimento formativo precoce e della dispersione scolastica attraverso specifiche azioni:

- di sostegno agli studenti con particolari difficoltà e fragilità perseguendo attività di tutoraggio ed integrative extra-orario scolastico, anche attraverso l'impiego di personale docente precario, nonché esperienze di stage (anche transnazionali) e laboratoriali;

- di sostegno alle famiglie anche attraverso la fruizione di risorse economiche, quali contributi libri di testo, buoni libro;

- di sostegno a percorsi di formazione di docenti e formatori per la messa in campo di metodologie innovative per il contrasto alla dispersione scolastica, con particolare riferimento a quelle aree o realtà scolastiche in cui il fenomeno presenta una più elevata rilevanza;

- di percorsi formativi di istruzione e formazione professionale;

- di orientamento in grado di supportare concretamente i processi di scelta dei giovani, favorendo un sistema orientativo di integrazione tra le politiche dell'istruzione, della formazione e del lavoro, in grado di accompagnare le famiglie, gli insegnanti e i formatori nelle attività di prevenzione e di lotta ai fenomeni di dispersione scolastica (ivi, pp. 32-33).

Il Piano considera inoltre prioritario l'innalzamento del livello di istruzione della popolazione adulta, attraverso:

- percorsi per giovani/adulti, in particolare per coloro che si trovano in situazioni di svantaggio, NEET, inoccupati, disoccupati, anche all'interno dei CPIA;

- percorsi per il conseguimento di una qualifica/diploma professionale o una qualificazione professionale (ivi, p. 33).

\subsection{Programma annuale per il diritto allo studio anno 2017}

Con un recente intervento legislativo teso a dare continuità alla precedente impostazione, la Regione Umbria, attraverso il Programma annuale per il diritto allo studio, assegna le risorse per l'assistenza scolastica ai Comuni, 
sulla base delle indicazioni contenute nel Piano triennale del diritto allo studio, come previsto dalla L.R. 28/2002.

Tale programma serve a sostenere e promuovere «processi di sperimentazione di rilevante contenuto didattico-pedagogico che coinvolgono insegnanti, famiglie e studenti e che hanno una significativa ricaduta nell'attività della scuola umbra» (Regione Umbria, Programma annuale per il diritto allo studio).

Il Piano triennale per il diritto allo studio attualmente in vigore è quello relativo al periodo 2013/2015. Come si legge nella delibera della Giunta Regionale che dà attuazione al Piano, in questo documento viene sollecitato un lavoro di forte «integrazione fra politiche educative, formative e del lavoro, compendiabili nel riferimento non giuridico delle "politiche dell'apprendimento", complessivamente rivolte a definire un insieme di tutele ed opzioni a supporto di tutte le transizioni che i singoli individui si trovano ad affrontare nel corso della propria vita attiva» (Regione Umbria, 2017b, p. 4).

Il Piano inoltre si inserisce nel quadro normativo europeo considerando principalmente la programmazione 2014-2020 dei Fondi strutturali comunitari, la quale «rende potenzialmente disponibili risorse mirate al tema del diritto allo studio, visto nella sua più ampia declinazione di supporto attivo agli apprendimenti in tutte le fasi della vita» (ibidem). In riferimento a questo aspetto si sottolinea un restringimento delle risorse pubbliche ordinarie che ha causato una «riduzione della significatività di parte degli interventi consolidati, con una possibile restrizione della platea dei beneficiari e/o della intensità dell'impegno» (ibidem).

Fra le aree tematiche prioritarie riportate nel Piano triennale emerge una forte attenzione al «contrasto alla dispersione scolastica nelle sue varie forme» (ivi, p. 5).

\section{Programmazione europea per la dispersione e ricadute territoriali}

\subsection{Fondo sociale europeo (FSE)}

Il Fondo sociale europeo (FSE) è lo strumento principale dell'Unione Europea (UE) per investire nelle persone (Cfr. Unione europea, 2016) attraverso l'erogazione e l'assegnazione di finanziamenti verso specifiche priorità, definite in maniera congiunta dalla Commissione europea con gli Stati membri dell'UE. 
Fra le sfide principali affrontate dall'FSE, forte attenzione è dedicata alla promozione dell' istruzione e dell'apprendimento permanente. A questo proposito la formazione professionale è considerata un elemento cruciale, soprattutto ai fini dell'inserimento nel mercato del lavoro.

Di primaria rilevanza è il tema della riduzione dell'abbandono scolastico. Il FSE indirizza ingenti risorse per contrastare l'interruzione degli studi e per promuovere l'acquisizione di titoli e certificazioni spendibili nel mercato del lavoro e ai fini di una professione. La questione della dispersione assume valori e numeri che non possono essere ignorati, meritano considerazione e interventi mirati, infatti

un giovane europeo su cinque (in totale, 6 milioni ogni anno) abbandona prematuramente gli studi e non completa il ciclo di istruzione secondaria: in questo modo, pregiudica le sue prospettive di carriera e qualsiasi possibilità di sfruttare al meglio le sue capacità (Unione europea, Porte aperte all'apprendimento).

Il FSE sostiene iniziative per prevenire la dispersione scolastica e nel contempo favorire competenze utili in ambito professionale. A tal riguardo vengono incoraggiati interventi e programmi innovativi che adottano approcci personalizzati per ogni singolo studente a rischio abbandono, ad esempio «consulenza e orientamento sulle proprie aspirazioni professionali e sulle qualifiche necessarie a conseguirle» (ibidem). Altra tipologia di azioni prevede formazione specifica per insegnanti o il coinvolgimento delle famiglie dei ragazzi a rischio o programmi dedicati a gruppi svantaggiati, come nel caso delle comunità di immigrati o delle minoranze Rom.

\subsection{PON 2014-2020 Programma Operativo Nazionale per la Scuola competenze e ambienti per l'apprendimento}

Il Programma Operativo Nazionale (PON) 2014-2020 del Ministero dell'Istruzione, dell'Università e della Ricerca, contiene le priorità strategiche in ambito di scuola e istruzione. È finanziato dai Fondi Strutturali Europei e in riferimento alla Programmazione 2014-2020 sono stati stanziati complessivamente 3 miliardi di Euro così ripartiti:

- «2,2 miliardi circa stanziati dal Fondo Sociale Europeo (FSE) per la formazione di alunni, docenti e adulti;

- 800 milioni dal Fondo Europeo di Sviluppo Regionale (FESR) per laboratori, attrezzature digitali per la Scuola e per interventi di edilizia» (MIUR, PON 2014-2020). 
Il PON si integra alla Strategia UE 2020 per un'economia intelligente, sostenibile e inclusiva, nonché per favorire occupazione, produttività e coesione sociale.

Sulla base delle Raccomandazioni specifiche del Consiglio Europeo, il Programma interviene sul contrasto alla dispersione scolastica, sostenendo il fine di ridurre l'abbandono degli studi, «incidendo sul successo formativo e sull'innalzamento dei livelli di istruzione» (MIUR, 2014, p. 2). Su questo fronte sono stati raggiunti notevoli risultati «con una significativa riduzione del tasso di abbandono prematuro degli studi, passato, nelle Regioni dell'Obiettivo convergenza, dal 26,4\% nel 2006 al 22\% nel 2013 (Fonte ISTAT)» (ivi, p. 5).

Nonostante i buoni traguardi raggiunti, la riduzione della dispersione scolastica e formativa rimane un obiettivo irrinunciabile, infatti il fenomeno raggiunge ancora valori fin troppo alti a livello nazionale, specialmente nelle regioni del Mezzogiorno e in alcuni contesti del Centro Nord. Nel PON si legge che «la percentuale di giovani in età 18-24 anni che abbandonano precocemente gli studi si attesta infatti, nel 2013, al 17\% a livello nazionale e al $21,4 \%$ per il Mezzogiorno (Fonte ISTAT), a fronte di un target del 10\% fissato per il 2020 dalla Strategia Europea e declinato al 15-16\%, quale obiettivo italiano, dal PNR» (ivi, p. 6). A questo proposito le raccomandazioni del Consiglio evidenziano la necessità «di intraprendere azioni più mirate e coordinate per affrontare la sfida dell'abbandono scolastico» (ibidem).

\subsection{Programma Operativo Regionale del Fondo Sociale Europeo 2014-2020}

Il Programma Operativo Regionale (POR) del Fondo Sociale Europeo (FSE) fornisce disposizioni per l'utilizzo dei finanziamenti stanziati dall'Unione Europa. In particolare,

contiene indicazioni riguardo l'ammontare delle risorse disponibili e i tipi di progetti per cui devono essere impiegate. Il Programma Operativo FSE 2014-2020 è approvato dalla Commissione Europea con un atto che si chiama decisione, nello specifico la Commissione Europea ha approvato il POR FSE Umbria con Decisione C (2014) 9916 del 12 dicembre 2014 (Regione Umbria, 2014b).

Nella strategia di azione del POR FSE sono individuati determinati bisogni che necessitano risposte operative in termini di intervento e che si traducono in Priorità di investimento rispetto alle Raccomandazioni Specifiche 
della UE per l'Italia. Fra i punti principali in ambito di istruzione e formazione viene promossa una riduzione dei tassi di abbandono scolastico. In particolare l'Obiettivo tematico "Investire nell'istruzione, nella formazione e nella formazione professionale per le competenze e l'apprendimento permanente», si prefissa come investimento prioritario, di «ridurre e prevenire l'abbandono scolastico precoce e promuovere l'uguaglianza di accesso a una istruzione prescolare, primaria e secondaria di buona qualità, inclusi i percorsi di apprendimento formale, non formale e informale, che consentano di riprendere l'istruzione e la formazione» (Regione Umbria, 2014a).

Il sostegno complessivo dell'UE per questo asse di intervento ammonta complessivamente a 28.334.336,00 di Euro. A livello europeo vengono quindi sensibilmente rafforzate le politiche nell'ambito del sistema integrato di Istruzione e Formazione Professionale per ridurre il fallimento formativo precoce e l'abbandono scolastico.

Gli interventi individuati e intrapresi in questo specifico settore sono prioritariamente rivolti ai giovani di età compresa fra 15 e 18 anni non compiuti, ancora impegnati ad assolvere l'obbligo di istruzione e a coloro «che abbandonano il sistema scolastico, verso i quali è rivolta la specifica offerta del sistema di formazione professionale, finalizzata al loro rientro stabile in percorsi di apprendimento professionale o finalizzati alla ripresa degli studi» (ivi, p. 86).

Le azioni operative propongono l'acquisizione e il consolidamento «di competenze di base, di cittadinanza e tecnico professionali» (ibidem), inoltre viene rivolta attenzione allo «sviluppo del capitale relazionale e alla valorizzazione degli apprendimenti maturati negli eventuali percorsi inconclusi, sotto forma di crediti formativi spendibili per il rientro nei dispositivi di formazione» (ibidem). In particolare, come si legge nel documento del POR FSE 2014-2020, gli interventi primari sono costituiti da:

- «offerta di percorsi formativi IeFP, in essi inclusi quelli rivolti al reinserimento di drop out da percorsi scolastici, da realizzarsi anche attraverso modalità di alternanza fra contesto di aula/laboratorio e contesto produttivo, con attenzione ai soggetti disabili e socialmente vulnerabili, anche sviluppabili negli ambiti e nelle tematiche proprie della green e blue economy;

- azioni di orientamento alle transizioni al termine della scuola secondaria di primo grado e in corso di studi» (ibidem).

Sono inoltre previste le seguenti tipologie di intervento:

a) osservazione del fenomeno della dispersione scolastica e formativa;

b) valutazione degli impatti delle politiche svolte e sviluppo dei raccordi fra istituzioni scolastiche, formazione professionale e servizi per il lavoro; 
c) promozione dei rapporti fra istituzioni scolastiche, organismi di formazione e aziende, anche al fine della comprensione delle logiche economiche e di funzionamento proprie delle imprese (Cfr. Regione Umbria, 2014a).

Le operazioni del POR FSE si integrano con il piano esecutivo regionale del PON Garanzia Giovani e sono pianificate in conformità del Piano triennale regionale per il diritto allo studio, al fine di evitare rischi di sovrapposizioni fra interventi.

\section{Dal Piano nazionale degli interventi e dei servizi sociali ai documenti di programmazione regionale umbra per pre- venzione, contrasto e azioni di recupero ${ }^{1}$}

\subsection{Primo e Secondo piano sociale. Azione unitaria sugli esiti della dispersione}

Il tema dell'abbandono degli studi ha acquisito un progressivo riconoscimento e una sempre maggiore attenzione da parte delle politiche territoriali di welfare, considerando il periodo di riferimento dal 2000, data dell'approvazione della L. 328 , la quale segna un punto di svolta per quanto riguarda la realizzazione del sistema integrato di interventi e servizi sociali, fino al 2017, anno in cui è stato approvato in Umbria il Nuovo Piano Sociale regionale.

Nei primi documenti di programmazione delle politiche sociali della Regione Umbria, l'abbandono scolastico non viene affrontato in via diretta, ma è indirettamente trattato all'interno della questione relativa all'inserimento lavorativo dei soggetti svantaggiati.

Analizzando i Piani sociali umbri del 2000 e del 2010, si riscontra un'attenzione maggiormente focalizzata sugli esiti della dispersione scolastica invece che sui fattori causali, quindi su effetti e danni dell'abbandono piuttosto che su traiettorie di prevenzione e interventi di contrasto al fenomeno.

Il Piano sociale 2000-2002, fra i suoi importanti contributi, sollecita e promuove un lavoro di integrazione e concertazione fra enti e istituzioni, attori e soggetti pubblici, del privato e del privato sociale di differente natura giuridica, anche anticipando le indicazioni della riforma costituzionale del

${ }^{1}$ Cfr. L. 328/2000, art. 18 comma 1 e comma 6, relativo al ruolo delle Regioni. 
Titolo V del 2001 (Cfr. L. Cost. 3/2001), dove è definito e ampiamente richiamato il principio di sussidiarietà. A questo proposito nel Piano del 2000 è evocato un lavoro di costruzione strategica di percorsi e di azioni:

Il complesso dei problemi indicati richiede politiche integrate che agiscono su diversi aspetti che concorrono a determinare la disoccupazione e chiama in causa ambiti diversi di intervento fra i quali (...) le politiche scolastiche, di inserimento dei soggetti a rischio di esclusione sociale, che operino per il diritto allo studio e l'integrazione scuola-lavoro (Regione Umbria, 1999).

Oltre a promuovere un approccio integrato per rispondere alle problematiche territoriali, nel documento del 2000 è individuata e riconosciuta una dimensione sociale nel fenomeno della disoccupazione, che rende opportuna una progettualità nuova nei provvedimenti operativi e negli interventi sociali rivolti alle «esigenze di socializzazione, formazione, accompagnamento, connesse alle finalità di inserimento/reinserimento nel lavoro, della centralità dell'integrazione e del coordinamento degli interventi per un'efficacia delle risposte» (ibidem).

Le azioni programmate hanno dimostrato un'alta innovazione metodologica nel lavoro di prevenzione, orientamento e integrazione sociale rivolto agli utenti per la promozione di «una rete territoriale di azioni e di collegamenti tra servizi, operatori, programmi, in grado di far efficacemente interagire soggetti istituzionali e non che possono contribuire allo sviluppo locale» (ibidem).

Nel primo Piano sociale il fenomeno della dispersione è implicitamente connesso al tema dell'inserimento lavorativo, pertanto le strutture operative di riferimento sono costituite dai Servizi di accompagnamento al lavoro (SAL) e dai Centri per l'inserimento lavorativo di soggetti deboli. Questo può essere indicativo di un'ottica che si focalizza sugli esiti dell'abbandono, quando i soggetti sono ormai fuori dal circuito scolastico e formativo, a conferma di un approccio maggiormente riparatorio ex post, piuttosto che preventivo ex ante o promozionale.

I Centri per l'inserimento lavorativo di soggetti deboli sono «servizi territoriali che operano per la prevenzione del disagio sociale e l'inserimento nel contesto socio-produttivo di soggetti deboli, attraverso attività di orientamento e sostegno all'incontro tra domanda e offerta di lavoro» (ivi, p. 127). Queste strutture si integrano con la rete dei servizi per l'impiego svolgendo consulenza alla persona in cerca di lavoro, grazie ad una équipe multiprofessionale costituita da operatori della mediazione e dell'orientamento. 
I destinatari ai quali si rivolgono sono tutti coloro che vivono «in condizione di svantaggio sociale in un particolare territorio, quali ad esempio, $\mathrm{i}$ ragazzi con abbandono scolastico e in situazioni di disagio socio-familiare e, più in generale, persone soggette a rischio di marginalità connessa a carenza di lavoro, a deficit formativi, in condizioni economiche, familiari, culturali di emarginazione sociale; immigrati in condizione di esclusione sociale» (ibidem).

Il Piano sociale regionale 2010-2012 mantiene e prosegue la direzione avviata dal 2000 per quanto riguarda le politiche riferite all'abbandono scolastico. Questo documento di programmazione si inserisce in un quadro di riferimento a livello comunitario, con la Politica Europea di Coesione 20072013, che «attribuisce all'Obiettivo Competitività Regionale e Occupazione la mission di supportare le regioni più sviluppate dell'Unione» (Regione Umbria, 2010, p. 126) anche attraverso la promozione di formazione e innovazione per una società della conoscenza, sulla base delle indicazioni e delle priorità di Lisbona (2000) e Goteborg (2001). In tale prospettiva diventa strategico investire in istruzione e formazione; pertanto il Piano dedica spazio e attenzione al tema «dell'apprendimento lungo tutto l'arco della vita e della formazione permanente che rappresentano uno strumento fondamentale per la competitività e l'occupabilità, per l'integrazione sociale, lo sviluppo delle forme della cittadinanza attiva e solidale, per l'autoproduzione sociale, la promozione dell'autorealizzazione dei cittadini, la qualificazione delle risorse formali e informali, espressione e patrimonio della comunità locale» (ivi, p. 130).

In definitiva temi come formazione, istruzione e lifelong learning hanno ottenuto progressivamente maggiore considerazione lungo lo sviluppo dei documenti di programmazione. Rispetto al problema della dispersione scolastica, le prospettive perseguite agiscono, in continuità con il Piano del 2000, attraverso un approccio riparatorio attivato per contrastare gli esiti dell'abbandono. Comunque il Piano sociale del 2010 rappresenta un lavoro scientifico-politico ampio, meticoloso, innovativo e capace di delineare traiettorie di sviluppo, ponendosi obiettivi coraggiosi di lungo periodo.

\subsection{Nuovo Piano sociale 2017-19}

Nel Nuovo Piano sociale del 2017 il tema della dispersione scolastica trova maggiore spazio e attenzione, rispetto ai precedenti documenti di pro- 
grammazione regionali. Oltre ad essere esplicitamente richiamato come termine, l'abbandono scolastico diventa oggetto di specifici programmi e interventi mirati, con l'obiettivo di arginare e prevenire tale problematica.

Nel Nuovo Piano l'impostazione delle risposte operative relative al fenomeno dell'abbandono è cambiata. Considerando il tipo di azioni rivolte al problema, è evidenziabile una evoluzione dai Piani sociali del 2000 e del 2010, con la quale si può riscontrare un passaggio da un'ottica riparatoria, che agisce prevalentemente in presenza del danno conclamato e rispondendo agli esiti del problema "abbandono scolastico", per perseguire un approccio preventivo e promozionale, che interviene prima che si verifichino casi di dispersione e abbandono degli studi.

$\mathrm{Si}$ assiste pertanto ad un graduale passaggio, anche grazie al contributo della ricerca sperimentale, verso ipotesi concrete di lavoro sulla dispersione in atto, rendendo l'abbandono scolastico asse di lavoro per le politiche territoriali di welfare, direttamente connesso alle dinamiche sociali educative, familiari e al tema dell'avviamento professionale.

La maggiore sensibilità culturale e l'attenzione operativa alla questione, è frutto anche di una più accorta recettività alle politiche europee e ai finanziamenti stanziati dai molteplici fondi comunitari, che erogano contributi per contrastare dispersione e abbandono nella UE. A tal proposto si sottolineano le difficoltà insite nella acquisizione dei finanziamenti europei, a volte causate da deficit di competenze progettuali finalizzate al reperimento di tali risorse. Anche per questo, nel Nuovo Piano sociale del 2017, maggiore attenzione è rivolta al tema delle politiche comunitarie e agli specifici stanziamenti del Fondo Sociale Europeo (FSE) e del Fondo Europeo di Sviluppo Regionale (FESR).

Per quanto riguarda le politiche per l'istruzione e la formazione, l'Umbria è stata tra le prime Regioni «ad aver riordinato il sistema formativo integrato, disciplinando le azioni per la promozione dell'orientamento, dell'istruzione e formazione professionale e delle politiche attive del lavoro» (Regione Umbria, 2017a, pp. 63-65). Le azioni per contrastare le criticità del sistema educativo e per garantire l'accesso alle opportunità formative elaborate a livello regionale possono essere individuate nei seguenti punti:

1. interventi sulle condizioni individuali di accesso e di permanenza nel sistema scolastico e di istruzione e formazione professionale (IeFP);

2. interventi sulla qualità dell'offerta educativa e di istruzione;

3. interventi di sistema (Cfr. Regione Umbria, 2017a, pp. 63-64).

Gli interventi sulle condizioni individuali di accesso, di permanenza nel sistema scolastico e di IeFP, si rivolgono a «destinatari caratterizzati da rischi 
di esclusione, da difficoltà di accesso o da possibilità di abbandono dei percorsi di istruzione e formazione» (ibidem). I Comuni, in forma singola o associata, promuovono interventi per assicurare «l'attuazione del diritto allo studio attraverso un'efficiente ed efficace organizzazione ed erogazione dei servizi» (ibidem), in virtù della Legge regionale 28/2002. Queste tipologie di azioni comprendono:

- erogazione di risorse economiche (buoni per acquisto di sussidi didattici, accesso a servizi collettivi, partecipazione ad offerta scolastica extracurricolare...) riservate a nuclei familiari definiti sulla base dell'ISEE, tenendo conto di particolari condizioni di numerosità e con riferimento alla frequenza di scuole secondarie di primo e secondo grado, pubbliche e paritarie;

- interventi a sostegno della dotazione di servizi collettivi di supporto al diritto allo studio.

Gli interventi sulla qualità dell'offerta educativa e di istruzione, mirano a soddisfare i bisogni di integrazione educativa e sociale e all'aumento dei tassi di partecipazione e di successo. Tutto ciò al fine di ridurre il tasso di abbandono degli studi e al contempo rafforzare l'autonomia delle istituzioni scolastiche, in una integrazione con l'offerta di formazione professionale. Mediante una programmazione annuale di settore, a livello regionale viene fornito sostegno:

- alle istituzioni scolastiche per l'inserimento e l'integrazione di studenti con disabilità, di cittadinanza non italiana o appartenenti a gruppi sociali potenzialmente a rischio di esclusione, agendo sulla dotazione di risorse professionali e didattiche, ivi inclusa la valorizzazione delle competenze del personale precario;

- ai CPIA per la promozione e la realizzazione dell'offerta educativa rivolta agli adulti a basso titolo di istruzione formale;

- al mantenimento del tempo scuola esteso e del tempo pieno, con specifico riferimento alla scuola primaria e alla scuola secondaria di I grado;

- all'innovazione pedagogica ed alla messa in rete - anche transnazionale - delle istituzioni scolastiche, mediante facilitazione istituzionale alla partecipazione a specifici progetti, in risposta ad iniziative nazionali e della Commissione Europea (ibidem).

La promozione del successo formativo e la riduzione del fenomeno della dispersione scolastica rappresentano alcune delle finalità poste dall'art. 2 della L.R. 7/2009 relativa al "Sistema Formativo Integrato Regionale". Questi propositi sono perseguiti grazie alla collaborazione tra i soggetti operanti nel territorio, pubblici, privati e del privato-sociale. 
In questo ambito, autonomia scolastica e didattica assumono ruoli centrali (Batini e Bartolucci, 2016) come elementi strategici e innovativi «per l'intervento di sostegno allo sviluppo dell'offerta formativa delle scuole» (ivi, p. 64). L'obiettivo regionale è incentivare i processi di qualità attraverso la progettazione di attività didattiche innovative, sperimentali e di integrazione territoriale già inseriti nei Piani dell'Offerta Formativa (POF) delle singole scuole o dei nuovi POF di territorio.

Gli interventi di sistema promossi nel Nuovo Piano sociale agiscono in un'ottica improntata all'integrazione fra tutti gli attori, istituzionali e non, che a vario titolo fanno parte del contesto sociale. Una concertazione virtuosa tra enti, istituzioni e terzo settore, è già sollecitata dai primi documenti di programmazione successivi alla L. 328/00 e servono a sviluppare «risorse comuni agli attori del sistema educativo e di istruzione e formazione professionale, in modo da accrescere, in una logica di investimento strutturale, la complessiva capacità di azione del sistema» (ibidem).

Viene evidenziato il necessario potenziamento delle esperienze di collaborazione tra scuola, mondo della cultura e mondo del lavoro per valorizzare modelli di apprendimento utili ed efficaci all'economia della conoscenza. In particolare gli interventi sono rivolti:

- «all'adeguamento e sviluppo delle risorse informative, con particolare riferimento all'anagrafe digitale degli studenti e del patrimonio edilizio scolastico, nonché all'osservazione del fenomeno della dispersione scolastica e formativa e dei tassi di successo. Le risorse informative sono un fattore essenziale di supporto alla programmazione degli interventi e alla valutazione dei relativi impatti. Occorre implementare l'Accordo operativo tra la Regione Umbria, il Ministero dell'Istruzione, Università e Ricerca e l'Ufficio Scolastico regionale per l'Umbria per la diffusione nelle scuole di ogni ordine e grado dei progetti e delle azioni di innovazione didattica, anche per accelerare lo sviluppo del Piano Nazionale Scuola Digitale;

- al rafforzamento del sistema dell'offerta di Istruzione e Formazione professionale;

- al rafforzamento dei servizi di orientamento finalizzati a supportare e accompagnare le scelte di studio dei giovani, a facilitare la transizione dal mondo dell'istruzione al mondo del lavoro, nonché a rilanciare l'istruzione tecnico-professionale e il contratto di apprendistato e a promuovere un uso più efficace dei tirocini formativi. Gli obiettivi sono: intervenire sulla riduzione dei tempi di transizione dal sistema dell'istruzione e della formazione a quello del lavoro; attivare interventi - anche attraverso la valorizzazione del ruolo degli attori pubblico-privati nel 
mercato del lavoro operanti sul territorio - volti a favorire lo sviluppo all'interno delle scuole e delle università di servizi di intermediazione $\mathrm{e}$ di politiche attive del lavoro, quali in particolare il tirocinio di formazione e orientamento e il contratto di apprendistato;

- al supporto agli schemi di apprendimento in alternanza, attraverso il sostegno alle relazioni fra istituzioni scolastiche e imprese, anche con riferimento all'evoluzione dei tirocini» (ivi, p. 65).

\section{Riferimenti bibliografici}

Archambault I., Janosz M., Dupéré V., Brault M.C. and Andrew M.M. (2017), "Individual, social, and family factors associated with high school dropout among low-SES youth: Differential effects as a function of immigrant status", British Journal of Educational Psychology, 87, 3: 456-477.

Azzolini D. and Barone C. (2012), "Tra vecchie e nuove disuguaglianze: la partecipazione scolastica degli studenti immigrati nelle scuole secondarie superiori in Italia”, Rassegna italiana di sociologia, 53, 4: 687-718.

Batini F. e Bartolucci M., a cura di (2016), Dispersione scolastica: ascoltare i protagonisti per comprenderla e prevenirla, FrancoAngeli, Milano.

Benvenuto G., a cura di (2011), La scuola diseguale: dispersione ed equità nel sistema di istruzione e formazione, Anicia, Roma.

Bernardoni A., Fazzi L. e Picciotti A. (2011), Welfare, innovazione e sviluppo locale. La cooperazione sociale in Umbria, Il Mulino, Bologna.

Bonomi S., Marcanti M. e Oppici F., a cura di (2014), Le formiche e l'elefante: una ricercaazione in sussidiarietà circolare per la prevenzione della dispersione scolastica. Aracne, Roma.

Checchi D., a cura di (2014), Lost: dispersione scolastica: il costo per la collettività e il ruolo di scuole e terzo settore, Ediesse, Roma.

Colombo M. (2010), Dispersione scolastica e politiche per il successo formativo: dalla ricerca sugli early school leaver alle proposte di innovazione, Erickson, Trento.

Commissione cultura scienza e istruzione $<7$. $>$, Sulle strategie per contrastare la dispersione scolastica: atti parlamentari 17. legislatura. Roma Camera dei deputati, 2014.

Unione europea (2016), Il fondo sociale europeo. Investire nelle persone, Ufficio delle pubblicazioni dell'Unione europea, Lussemburgo.

Cozzolino M., a cura di (2014), Motivazione allo studio e dispersione scolastica: come realizzare interventi efficaci nella scuola, FrancoAngeli, Milano.

Devastato G. (2012), Oltre la crisi. Quali sfide per il welfare dei soggetti, Maggioli Ed., Santarc. di Romagna.

Fadda R. e Mangiaracina E., a cura di (2011), Dispersione scolastica e disagio sociale: criticità del contesto educativo e buone prassi preventive, Carocci, Roma.

Ferrera M., Fargion V. e Jessoula M. (2012), Alle radici del welfare all'italiana. Origini e futuro di un modello sociale squilibrato, Marsilio, Venezia.

* Si ringrazia il Dott. Marco Capacci per la complessa ricerca normativa e documentaria. 
Grimaldi E., Romano T. e Serpieri R. (2011), prefazione di Cortese A., I discorsi della dispersione: una politica di partenariato, Liguori, Napoli.

Menzani T. (2015), Cooperative: persone oltre che imprese, Rubettino, Soveria Mannelli.

MIUR, PON 2014-2020, testo disponibile al sito: in http://www.istruzione.it/pon/ilpon.html.

MIUR (2014), PON 2014-2020, Per la Scuola-competenze e ambienti per l'apprendimento, CCI: 2014IT05M2OP001 - Decisione C (2014) 9952.

Mollo G., Porcarelli A. e Simeone D. (2014), Pedagogia sociale, Editrice La Scuola, Brescia.

Pellerone M., a cura di (2015), Rischio di dispersione scolastica e disagio socio-educativo: strategie e strumenti di intervento in classe, FrancoAngeli, Milano.

Premoli S., a cura di (2008), Il coordinamento pedagogico nei servizi socioeducativi, FrancoAngeli, Milano.

Programma operativo nazionale 2000-2006 - OB. 1: 1999 IT 05 1 PO 013. La scuola per lo sviluppo: alla ricerca della qualità. Rapporto sulle scuole che operano per promuovere il successo scolastico: misura c3.2 - Interventi per la prevenzione e il recupero della dispersione scolastica, RTI union contact, 2012.

Regione Umbria (1999), Piano Sociale 2000-2002, approvato con deliberazione del Consiglio Regionale 20 dicembre 1999, n. 759.

Regione Umbria (2010), Piano Sociale 2010-2012, approvato con deliberazione del Consiglio Regionale 19 gennaio 2010, n. 368.

Regione Umbria - Servizio Istruzione, Università e Ricerca (2013), Piano Triennale per il diritto allo studio 2013-2015, approvato con deliberazione dell'Assemblea Legislativa 17 dicembre 2013 n. 294.

Regione Umbria (2017a), Nuovo Piano Sociale Regionale 2017-2019, approvato con deliberazione dell'Assemblea Legislativa 7 marzo 2017, n. 156.

Regione Umbria, Programma annuale per il diritto allo studio, testo disponibile al sito: http://www.regione.umbria.it/istruzione/programma-annuale-diritto-allo-studio-contributi-per-iniziative-progettuali-in-materia-di-istruzione.

Regione Umbria (2017b), Deliberazione della Giunta Regionale N. 1086, Oggetto: Programma annuale per il diritto allo studio anno 2017, seduta del 28/09/2017.

Regione Umbria (2014a), POR FSE 2014/2020 Obiettivo: "Investimenti a favore della crescita e dell'occupazione". Decisione di esecuzione della Commissione C (2014) 9916 del 12.12.2014 CC1 2014IT05SFOP010.

Regione Umbria (2014b), POR FSE Umbria 2014-2020, testo disponibile al sito: http://www.regione.umbria.it/lavoro-e-formazione/il-por-fse-umbria-2014-2020.

Rossi-Doria M. e Tabarelli S., a cura di (2016), Reti contro la dispersione scolastica: i cantieri del possibile, Erickson, Trento.

Sandomenico C. (2010), Le maschere della dispersione scolastica: riflessioni intorno all'evoluzione del fenomeno ed ai suoi profili attuali, Edup, Roma.

Tolomelli A. e Guerzoni G., a cura di (2017), Per non perdere la strada...: progetto ATOMS: fare rete tra teorie ed esperienze nel contrasto alla dispersione scolastica e formativa, CLUEB, Bologna.

Triani P., Ripamonti E. e Pozzi A., a cura di (2015), Centra la scuola: interventi di sistema per la grave dispersione scolastica, Vita e pensiero, Milano.

Unione europea, Porte aperte all'apprendimento, testo disponibile al sito: http://ec.europa.eu/esf/main.jsp?catId=528\&langId=it.

Unione europea (2016), Il Fondo sociale europeo. Investire nelle persone, Lussemburgo, testo disponibile al sito: in http://ec.europa.eu/esf/main.jsp?catId=3\&langId=it. 


\title{
Una valutazione per il successo formativo
}

\author{
di Cristiano Corsini
}

\author{
Well, we busted out of class \\ Had to get away from those fools \\ We learned more from a three-minute record, baby \\ Than we ever learned in school \\ B. Springsteen, No surrender
}

\section{Narrazione e valutazione}

Sussistono pochi dubbi sull'utilità di una didattica basata in modo appropriato su prassi narrative. Tale approccio, d'altra parte, si pone in aperta continuità rispetto a una tradizione che richiama apertamente l'attivismo pedagogico e, incentrandosi sulle esperienze di chi apprende, supporta, attraverso un'attiva semanticizzazione linguistica delle stesse (Veggetti, 2016), l'impiego di processi psichici superiori e il loro sviluppo, oltre a favorire l'emergere di una motivazione intrinseca all'apprendimento. Si tratta di apprendere a conferire senso all'esperienza pregressa e presente per orientare quella futura. In particolare, l'orientamento narrativo si propone, attraverso lo sviluppo di competenze narrative (capacità di ascoltare, interpretare, attribuire significati e narrare), di incidere positivamente sul senso di autoefficacia e di autodeterminazione individuale, giacché orientare chi apprende «significa trasferirgli competenze di autorientamento, con la finalità di scelte (il plurale è d'obbligo) immediate o future, in direzione di una decisione o per una lettura più appropriata di un contesto esistenziale e/o professionale, per progettare un percorso formativo o per migliorare la percezione di sé in direzione di un'efficacia maggiore nell'azione di soddisfacimento dei propri bisogni, di realizzazione dei propri progetti e desideri, in direzione di una maggiore chiarezza su questi stessi» (Batini e Del Sarto, 2007, p. 48).

Non stupisce dunque che l'efficacia delle pratiche narrative nel contrasto alla dispersione scolastica stia ricevendo il supporto di crescenti evidenze empiriche (Batini, Bartolucci e De Carlo, 2017). Il presente contributo intende offrire una riflessione relativa, in primo luogo, all'approccio valutativo coerente con una didattica siffatta e, in seconda istanza, agli ostacoli che si frappongono a una apprezzabile diffusione di tale approccio. 


\subsection{La valutazione autentica}

Secondo l'antropologa Michèle Petit, uno dei vantaggi di un vivo e approfondito rapporto con la lettura e la narrazione è quello di imparare a «non essere solo oggetto di valutazione». Il ricorso a dinamiche valutative per esprimere l'emergere di una proattiva capacità di attribuzione di significato alla realtà nella quale ciascuna persona è immersa è del tutto ragionevole dal punto di vista semantico e lessicale, giacché valutare significa conferire valore. In aggiunta, tale ricorso appare felicemente fondato se raffrontato con lo sviluppo, nel corso degli ultimi decenni, dell'orientamento valutativo che verrà sinteticamente presentato in questo contributo.

Nel 1998, Wiggins pubblica "Educative Assessment". Nel lavoro l'autore, sulla scorta di una critica a un certo uso del testing (Wiggins, 1993), definisce autentico un compito sulla base di prerogative quali l'essere realistico (ricostruendo il modo in cui conoscenze e abilità oggetto d'insegnamento possono essere testate nella vita reale), il richiedere l'impiego creativo di conoscenze per affrontare situazioni complesse, l'implicare il ruolo attivo di chi apprende nella ricostruzione dei saperi disciplinari, il simulare la complessità e il disordine dei contesti extrascolastici, valutando l'uso di abilità e conoscenze come strumenti da impiegare per risolvere le situazioni problematiche insorgenti in tali contesti e, infine, l'offrire la possibilità di impiegare il feedback come strumento trasparente di apprendimento nel corso delle attività. Così, mentre nella valutazione tradizionale elementi chiave (come le specifiche nozioni testate o le relative domande) non vengono notificati in anticipo, nella valutazione autentica è essenziale che lo siano, giacché essi rappresentano gli strumenti che potranno consentire soluzioni possibili ai problemi complessi da affrontare. La stessa valutazione, non mirando all'erogazione di punteggi o classificazioni, ma a un feedback relativo al posizionamento su una scala di padronanza e finalizzato a garantire prestazioni, processi e prodotti di qualità, diviene momento (anche autoregolativo) di sviluppo e consolidamento di conoscenze e abilità centrali per le discipline scolastiche.

Wiggins propone una tripartizione della valutazione scolastica sulla base di una progressiva autenticità (letta come "questione di gradi"). L'autore procede valutazioni "inautentiche" ad "autentiche" passando per valutazioni "parzialmente realistiche". Nello specifico, vengono presentati esempi di tale tripartizione in merito all'ambito scientifico, sociale e alla lettura. L'argomento di questo convegno porta a focalizzare l'attenzione su quest'ultima tripartizione, riportata nella tabella 1. 
Tab. 1 - Gradi di autenticità della valutazione (Wiggins, 1998)

\begin{tabular}{|l|l|l|}
\hline Inautentico & $\begin{array}{l}\text { Parzialmente } \\
\text { realistico }\end{array}$ & Autentico \\
\hline $\begin{array}{l}\text { Leggere } \\
\text { un passo } \\
\text { di un testo } \\
\text { scelto dal } \\
\text { docente. }\end{array}$ & $\begin{array}{l}\text { Leggere alla } \\
\text { classe un testo } \\
\text { scelto autono- } \\
\text { mamente. }\end{array}$ & $\begin{array}{l}\text { Produrre un audio- } \\
\text { libro da fornire a } \\
\text { una biblioteca. }\end{array}$ \\
\hline
\end{tabular}

Questi tre casi, e in particolare il primo e il terzo, fanno riferimento a strategie didattiche assai distanti fra loro. In quella che Wiggins definisce situazione inautentica, la valutazione segue l'insegnamento come suo momento separato. Nello specifico, si chiede di leggere ad alta voce una parte di testo scelta da altri. Perché definire inautentica tale scelta didattica? Come già rilevato (Batini e Corsini, 2016), si tratta di una valutazione inautentica perché, direbbe Pennac nel suo "Diario di scuola", lo studente non risponde alla domanda posta, ma al fatto che gli venga posta. Quanto incide sulla prestazione tale inautenticità, rendendo difficile per chi viene messo alla prova richiamare alcune nozioni sul testo stesso, sulla punteggiatura, come strumenti per affrontare la situazione? Non poco. La mobilitazione di risorse, cognitive e non, è parzialmente ostacolata sia dall'artificiosità della situazione sia dallo stress apportato dalla funzione della valutazione, la cui posta in palio è, spesso, meramente un voto, un punteggio che rimanda sinteticamente, in maniera non sempre affidabile e trasparente, al livello di apprendimento raggiunto e che, non infrequentemente, viene espresso in termini di distanza dai valori medi di un gruppo.

Le cose iniziano a cambiare passando al cosiddetto compito realistico: a chi apprende è affidata la scelta del testo da leggere e viene dato del tempo per preparare la lettura. L'idea di partire dagli interessi di chi apprende e la richiesta di autonoma organizzazione del compito possono conferire maggiore significatività allo sforzo di apprendimento, favorendo il ricorso ad adeguate strategie di autoregolazione dell'apprendimento, con il richiamo di elementi cognitivi e metacognitivi.

Infine, con la valutazione autentica, siamo di fronte all'approntamento di "prodotti" che conservano significato al di là della situazione didattica e che, per loro natura, possono prevedere un apprendimento cooperativo, incentrato su dinamiche di interdipendenza positiva tra più individui. Si tratta dunque di percorsi caratterizzati non solo da un maggior grado di prossimità alle esperienze e agli interessi di chi apprende ma, soprattutto, dall'inserimento 
autentico degli esiti (in questo caso, un audiolibro consultabile in una libreria) in contesti reali. L'approntamento di tali prodotti pone problemi complessi la cui soluzione (non univoca né scontata) è facilitata proprio dalla mobilitazione delle conoscenze e delle capacità oggetto di insegnamento e apprendimento: ciò facilita un apprendimento significativo.

Mentre nella valutazione tradizionale insegnamento e valutazione sono polarità nettamente circoscritte e rigidamente separate, dato che la valutazione tende a seguire l'intervento di chi insegna ratificandone il grado di successo, nella valutazione autentica il momento valutativo assume un ruolo fondamentale come elemento di regolazione tanto dell'apprendimento quanto dell'insegnamento, assolvendo la funzione di fornire feedback utili nel corso dell'attività. Prende così forma la distinzione tra valutazione dell'apprendimento e valutazione per l'apprendimento, esplicitata da Black e Wiliam nel 1998. Nella valutazione per l'apprendimento la rilevanza del momento valutativo all'interno del processo didattico comporta l'impiego di strumenti adeguatamente rigorosi, descrittori analitici che, per mezzo di una trasparente e affidabile esplicitazione dei livelli di padronanza, siano in grado di fornire a docenti, studentesse e studenti la possibilità di regolare insegnamento e apprendimento. Questo spiega la centralità assunta dalle rubriche di competenza e di prestazione.

\subsection{Valutazione, motivazione e apprendimento}

Un certo grado di inautenticità è fondamentale nelle faccende scolastiche e accademiche, dato che l'istruzione necessita di mediazione (e non è un caso che "scolastico" e "accademico" significhino entrambi, a modo loro, "inautentico"). Lo stesso Wiggins si guarda bene dal proporre un'istruzione interamente incentrata su compiti autentici e sostiene, piuttosto, la complementarietà tra valutazione autentica e inautentica. Non si tratta dunque di immaginare una scuola fondata sull'apprendistato, ma di rilevare come la questione sia soprattutto un fatto di dose, e che tradizionalmente la scuola tenda a esagerare con l'inautenticità. Un'inautenticità che, separando nettamente insegnamento e valutazione, scoraggia l'impiego di questa come strumento di autoregolazione dell'apprendimento, e assegna buone possibilità di riuscita soprattutto a chi è già portato in particolare per quella disciplina $\mathrm{o}$, in generale, per lo studio. Inoltre, una didattica del tutto inautentica rischia di incentivare un rapporto formale ed estrinseco con la disciplina, con i contenuti, con la competenza da sviluppare, finendo col favorirne l'identificazione 
con i patemi vissuti durante la valutazione e incidendo negativamente sull'impegno verso futuri compiti di apprendimento.

Il rapporto tra valutazione e motivazione all'apprendimento è una delle determinanti del successo scolastico che maggiormente chiamano in causa l'agire didattico. È principalmente alle indagini condotte da oltre tre decenni (Elliott e Dweck, 1988) che si deve la distinzione tra obiettivi di padronanza e di prestazione. Nella tabella 2, tratta da Watkins (2001), si offre una schematica e utile distinzione tra orientamento di studentesse e studenti alla prestazione (ovvero alla dimostrazione delle proprie abilità e/o all'evitamento di prestazioni negative), associato a una motivazione estrinseca nei confronti dell'apprendimento (percepito come obiettivo imposto dall'esterno e da altri) e orientamento alla padronanza (associato a una motivazione intrinseca verso l'apprendimento, ovvero allo sviluppo delle proprie competenze).

Tab. 2 - (Watkins, 2001)

\begin{tabular}{|l|l|}
\hline $\begin{array}{l}\text { "Learning orienta- } \\
\text { tion": concern for im- } \\
\text { proving one's compe- } \\
\text { tence }\end{array}$ & $\begin{array}{l}\text { "Performance orienta- } \\
\text { tion": concern for pro- } \\
\text { ving one's competence }\end{array}$ \\
\hline $\begin{array}{l}\text { belief that effort leads to } \\
\text { success }\end{array}$ & $\begin{array}{l}\text { belief that ability leads to } \\
\text { success }\end{array}$ \\
\hline $\begin{array}{l}\text { belief in one's ability to } \\
\text { improve and learn }\end{array}$ & $\begin{array}{l}\text { concern to be judged as } \\
\text { able, concern to perform }\end{array}$ \\
\hline $\begin{array}{l}\text { preference for challen- } \\
\text { ging tasks }\end{array}$ & $\begin{array}{l}\text { satisfaction from doing bet- } \\
\text { ter than others }\end{array}$ \\
\hline $\begin{array}{l}\text { derives satisfaction from } \\
\text { personal success at diffi- } \\
\text { cult tasks }\end{array}$ & $\begin{array}{l}\text { emphasis on normative } \\
\text { standards, competition and } \\
\text { public evaluation }\end{array}$ \\
\hline $\begin{array}{l}\text { uses self-instructions } \\
\text { when engaged in task }\end{array}$ & $\begin{array}{l}\text { helplessness: evaluate self } \\
\text { negatively when task is dif- } \\
\text { ficult }\end{array}$ \\
\hline
\end{tabular}

A differenza di quanti sono orientati verso obiettivi di prestazione, studentesse e studenti orientati alla padronanza tendono a considerare la riuscita nel compito di apprendimento in rapporto a se stessi (allo sviluppo delle proprie competenze), a ritenere che i risultati raggiunti siano associati all'impegno profuso e a sviluppare un adeguato senso di autoefficacia, perseverando in comportamenti finalizzati al perseguimento di scopi percepiti come intrinsecamente interessanti. Studentesse e studenti orientati alla prestazione considerano la riuscita in rapporto agli altri, posizionando i criteri di successo o insuccesso all'esterno. 
Come accennato, quanto evidenziato dalla psicologia dell'apprendimento riguardo alle relazioni tra motivazione e apprendimento chiama in causa l'agire didattico. Nel 1992 Carole Ames pubblica "Classroom: Goals, structures and student motivation", evidenziando l'incidenza di alcune scelte operate da chi insegna nell'orientare chi apprende verso una motivazione intrinseca o estrinseca. Ames sottolinea come studentesse e studenti arrivino in classe con una motivazione verso certe discipline fortemente condizionata dalla famiglia e/o dalle esperienze scolastiche precedenti. Ma chi insegna non è impotente, giacché studentesse e studenti percepiscono l'enfasi posta da docenti su obiettivi di padronanza o di prestazione. In particolare, la ricercatrice evidenzia l'associazione tra determinate scelte valutative e lo sviluppo di orientamenti verso l'apprendimento. Orienta verso obiettivi di prestazione una valutazione che enfatizza l'assenza di errori e scoraggia la collaborazione, che incentra il giudizio (facendo riferimento a una norma) sul confronto con le prestazioni altrui, che ha come posta in palio premi o punizioni e viene gestita completamente dall'alto. Al contrario, a orientare verso obiettivi di padronanza, è una valutazione che usa gli errori nella didattica e incoraggia momenti di collaborazione e insegnamento tra pari, basata sui progressi di ciascuno stabiliti in termini di padronanza (facendo riferimento a un criterio e non al posizionamento rispetto a un gruppo), che ha come posta in palio indicazioni per lo sviluppo e viene cogestita da chi insegna e chi apprende.

Come accennato, l'approccio proposto segnala l'urgenza di un passaggio da una mera valutazione dell'apprendimento (assessment of learning) a una valutazione per l'apprendimento (assessment for learning) (cfr., fra gli altri, Black et al., 2004; Stiggins, 2002; Greenstein, 2010; Swaffield, 2011). Si tratta di una valutazione progettata e finalizzata allo scopo di promuovere l'apprendimento attraverso feedback che procedono tanto da chi insegna quanto da chi apprende, in modo tale da apportare, se necessario, modifiche alla didattica, e garantire a studentesse e studenti una progressiva padronanza nella gestione del proprio apprendimento. Da questo punto di vista, la valutazione per l'apprendimento si pone coerentemente nel solco della valutazione formativa, che è tale se dà forma all'insegnamento. Ma, al tempo stesso, come emerso sulla base di quanto individuato da Wiggins e Watkins, la valutazione per l'apprendimento rappresenta un'evoluzione della valutazione formativa, un suo arricchimento alla luce delle teorie dell'apprendimento.

Un ulteriore passaggio evolutivo si riscontra nel concetto di assessment as learning, introdotto da Earl (2013) e traducibile (Trinchero, 2018) come "valutazione formante". Si tratta di una forma di valutazione che si concentra 
sul ruolo dello studente come connettore critico tra la valutazione e il proprio apprendimento. Un processo di regolazione metacognitiva che si verifica quando chi apprende monitora ciò che sta imparando e utilizza il conseguente feedback per conferirvi senso, mettendolo in relazione con le conoscenze pregresse e facilitando la costruzione attiva di nuove conoscenze (Earl, 2013, p. 47). La valutazione formante (Trinchero, 2018) si incentra su esplicite richieste valutative sin dall'inizio del processo didattico in funzione di attivazione cognitiva, ovvero di recupero e utilizzo da parte di studentesse e studenti delle proprie risorse cognitive e metacognitive allo scopo di conferire significato alle esperienze di apprendimento. Una dinamica del tutto coincidente con l'obiettivo, richiamato in apertura, di una didattica basata su prassi narrative: apprendere a conferire senso all'esperienza pregressa e presente per orientare quella futura.

\section{Le culture della valutazione}

Nel 1998 Black e Wiliam pubblicano "Inside the black-box". L'articolo sottolinea lo scollamento tra le scelte didattiche di buona parte del corpo docente e l'evidenza empirica raccolta dalla letteratura scientifica sull'efficacia della valutazione formativa. Secondo gli autori c'è in primo luogo un problema in relazione alla finalità della valutazione: «teachers' feedback to pupils seems to serve social and managerial functions, often at the expense of the learning function». In poche parole, la valutazione assolve funzioni di tipo burocratico/disciplinare piuttosto che educativo. Si valuta perché si deve, ed è saggio farlo dopo più valutazioni, e si usano spesso le valutazioni come minaccia per mantenere la disciplina in classe. In estrema sintesi, l'articolo evidenzia che

1) si ricercano valutazioni oggettive, numeriche e sintetiche, trascurando il fine di dare informazioni per migliorare l'apprendimento;

2) c'è una spinta verso la memorizzazione più che verso la comprensione: la preoccupazione per i voti porta a un apprendimento mnemonico e i contenuti dopo l'esame vengono spesso dimenticati;

3) è assente condivisione tra i docenti di modalità valutative;

4) c'è maggiore attenzione alla quantità che alla qualità (dei voti e dei lavori): i docenti sono preoccupati più dal numero delle valutazioni che dalla loro significatività;

5) la valutazione finisce spesso col demotivare verso lo studio. 


\subsection{La cultura della valutazione emergente}

Il quadro delineato da Black e Wiliams venti anni fa può essere tuttora considerato una vivida descrizione della situazione attuale. Questo, a dispetto dei richiami alla diffusione di una cultura della valutazione che negli ultimi anni hanno caratterizzato tanta letteratura e tanta normativa. Per comprendere meglio tale fenomeno è necessario ragionare sulle dimensioni che hanno caratterizzato l'affermazione di tale cultura. Cosa si intende per cultura della valutazione? Nonostante la locuzione sia ampiamente richiamata, non è facile rintracciarne una compiuta definizione. Nel rapporto Invalsi sull'indagine Pirls 2006 (Invalsi, 2008, p. 99) si parla di «sviluppo di una cultura della valutazione (teach to test) con l'introduzione nelle scuole di prove di verifica strutturate (quesiti a scelta multipla) e semistrutturate (quesiti con stimolo chiuso e risposta aperta)». Si tratta di una definizione fortemente incentrata sugli strumenti, sebbene la definizione di una cultura, anche in un campo in cui l'utilizzo di strumenti di misura è distintivo, non possa evitare riferimenti espliciti o rimandi impliciti a valori, credenze, finalità. D'altra parte gli stessi indicatori, come ricordano Fitz-Gibbon e Kochan (2000), si riferiscono a «things we care about enough to measure».

Da questo punto di vista, dal punto di vista del perché dei processi valutativi che troppo spesso è adombrato dal come e dal cosa, l'attuale cultura della valutazione viene assimilata all'uso di strumenti rigorosi, dato che essi garantiscono oggettività alla misurazione dell'efficacia di scuole, università e docenti. A tanto rigore la cultura della valutazione assegna l'obiettivo di tenere sotto controllo le prestazioni di studentesse e studenti, scuole e università, verificando il risultato degli investimenti realizzati. La cultura della valutazione si sviluppa come aspetto complementare dell'autonomia scolastica e universitaria, descrivendosi prevalentemente in termini di accountability, ovvero di rendicontazione/rendicontabilità. Una cultura valutativa che si basa su quella che Lucisano $(2011,2015)$ definisce Sindrome del Figlio dell'Uomo, ovvero sull'idea che al controllo, alle misure e ai giudizi non possano che far seguito miglioramenti.

Una cultura valutativa caratterizzata da un ingenuo induttivismo, ben rappresentato dalle parole con le quali l'allora ministro Tremonti salutava il passaggio dai giudizi ai voti nella scuola primaria: «i numeri sono una cosa. I giudizi sono una cosa diversa. I numeri sono una cosa precisa, i giudizi sono spesso confusi. Ci sarà del resto una ragione perché tutti i fenomeni significativi sono misurati con i numeri [...]. I numeri sono insieme precisi e semplici. Il messaggio che trasmettono è un messaggio diretto. Se gli stessi fenomeni - terremoto, moto marino, pendenza, temperatura corporea - fossero 
espressi non con numeri ma attraverso frasi complesse con finalità descrittive, il messaggio resterebbe impreciso» (Il passato e il buon senso, in "Corriere della Sera", 22 agosto 2008).

Questo induttivismo incolto, che ignora la visione dialettica del rapporto tra misurazione e giudizio di valore proposta nei decenni precedenti (Visalberghi, 1955), assume un ruolo centrale, dato che garantisce inappellabilità alle classifiche stilate e date in pasto a un'opinione pubblica ansiosa di capire quali scuole (o università) facciano il "proprio dovere" (figura 1). Vengono ampiamente impiegati indicatori che danno luogo a classifiche e finiscono col produrre, nell'immaginario collettivo, un sostanziale rovesciamento dei rapporti tra scuola e società così come erano stati delineati da Don Milani, dal Rapporto Coleman e dai successivi decenni di ricerche empiriche in educazione, addossando su scuole e docenti l'intera responsabilità delle differenze negli apprendimenti. Una sorta di teodicea secolare, che assolve il sistema economico dalle sue storture scaricandole su scuole e docenti. Un processo ben rappresentato dal calcolo del valore aggiunto rilevato dall'Invalsi o dalla classifica Eduscopio delle scuole migliori operata dalla Fondazione Agnelli. Si tratta di indicatori del tutto privi di validità (Amrein-Beardsley, 2014; Asa, 2014; Baker et al., 2010; Corsini 2009, 2012), dato che non v'è corrispondenza alcuna tra le misure cui danno luogo e il costrutto che dovrebbero rappresentare, ovvero la qualità dell'insegnamento (Corsini e Zanazzi, 2015). Per esempio, l'ambiziosa e pluridecennale pretesa del valore aggiunto di ricondurre al residuo di una regressione l'efficacia e la qualità dell'insegnamento (attribuendo dunque a scuole e docenti la quota di varianza non spiegata da una funzione di produzione educativa) non ha prodotto alcuna evidenza empirica affidabile a suo sostegno e fatica a venire a patti con un costrutto che rimane elusivo se non assente. 


\section{CORRIERE DELLA SERA Invalsi 2016: il record di Trento, l'exploit di Marche e Umbria, il ritardo cronico del Sud \\ Decolla il nuovo «valore aggiunto» che calcola la qualità di una scuola misurando i livelli di partenza e di arrivo dei ragazzi al netto del contesto socio-demografico di riferimento. \\ Al Sud record negativo di scuole che non fanno il proprio dovere}

Fig. 1 - Il titolo del "Corriere della Sera" sul valore aggiunto calcolato dall'Invalsi

Tali meccanismi valutativi non sono meno nocivi dentro il sistema educativo. Prendendo in prestito i concetti espressi da Foucault in "Soggettività e verità", siamo in presenza della costruzione di una verità, intesa come sistema di obblighi, che affermandosi porta i soggetti a interiorizzare meccanismi che li depotenziano. Secondo Borrelli (2015) e Pinto (2012, 2014), tali dinamiche sono tanto più pervasive quanto più anonime e numericamente espresse. Questa cultura valutativa comporta dunque effetti deleteri sulla qualità della didattica e sui processi d'inclusione (cfr., tra gli altri: Baker et al., 2010, Fondazione Giovanni Agnelli, 2014; Koretz, 2009; Ravitch, 2010), come il teaching to the test (dedicare sforzi, spazi e tempi didattici nell'addestrare studentesse e studenti alla forma del test), il cheating (agevolare le risposte esatte durante la somministrazione di test), lo skimming (selezionare per il test solo i soggetti migliori, allontanando quelli ritenuti peggiori o richiedendo per essi particolari certificazioni). La posta in palio legata alle misure (reputazione di dirigenti, scuole e docenti, possibilità di attrarre iscrizioni e fondi) può comportare la tendenza a rafforzare meccanismi in ingresso o in itinere della popolazione studentesca, in modo tale, ad esempio, da consentire alla scuola di ottenere posizioni migliori nella classifica Eduscopio, incentrata sul rendimento di ex studenti al primo anno di università. Elementi non ritenuti inclini ad affrontare un buon percorso accademico potrebbero essere scoraggiati nel proseguire gli studi in quello stesso istituto.

Più in generale, una cultura della valutazione concepita in termini di accountability rende scarsamente conveniente includere e consolida la propensione a leggere le difficoltà incontrate da studentesse e studenti, o l'eterogeneità della classe, in termini di problemi da evitare. 
La recente polemica sui "licei classisti", innescata dalla pubblicazione di Rapporti di Autovalutazione (Rav) che associano la qualità di apprendimento e insegnamento alla scarsa presenza di stranieri e disabili, assume da questo punto di vista una duplice rilevanza. In primo luogo conferma quanto i meccanismi di accountability possano rafforzare la tendenza a pensare le differenze come deficit. La seconda considerazione è relativa alla scelta di alcune scuole di difendersi dall'assalto mediatico cui sono state sottoposte, asserendo che quanto scritto sarebbe meramente in linea con la richiesta di fornire dati statistici. Ma, intanto in quei Rav c'è di più, ovvero una interpretazione di quei dati ben precisa e non richiesta, un'interpretazione che associa, implicitamente o esplicitamente, la qualità di processi di apprendimento e insegnamento all'ambiente elitario. E questa interpretazione (del tutto coerente con la teodicea secolare prodotta da questa cultura della valutazione), sta in un documento che le famiglie usano per scegliere la scuola. Ma, soprattutto, il richiamo ai dati è un buon esempio di quanto, all'interno di questa cultura valutativa, il come e il cosa nascondano il perché della valutazione. Tali informazioni, richieste dal sistema legato al dpr 80/2013 e fornite dalle scuole (che tuttavia possono anche scegliere di sottrarle al pubblico) vengono presentate come elementi primitivi, genuini, come dati, appunto. Ma i dati, scrive Dewey nella Logica, sono sempre assunti da chi fa ricerca come fatti del caso, elementi significativi nel corso dell'indagine; per usare le parole di Kuhn, «la teoria paradigmatica è direttamente implicita nel progetto dell' apparecchiatura capace di risolvere il problema» (Kuhn, 1962, p. 47). Un sistema che consente alle scuole di pubblicare certi dati è un sistema che assume che sia giusto che le famiglie scelgano una scuola in base al numero di stranieri o disabili che la frequentano.

\subsection{Un'altra cultura della valutazione}

L'affermazione della cultura della valutazione sin qui descritta appare in inarrestabile espansione, con Invalsi e Anvur che condizionano una parte via via crescente del lavoro di scuole e università. Non sembra esserci alternativa a questa cultura della valutazione. Ma, se è vero che ci accorgiamo dell'esistenza di una cultura quando avvertiamo l'esistenza di un'alternativa a quella cultura, appare appropriato delineare una cultura valutativa alternativa o, per meglio dire, autentica. Ricondotta l'emergente cultura della valutazione nell'ambito dell'accountability, rimane da vagliare se essa abbia o meno diritto di cittadinanza all'interno della riflessione più fondata sui processi valutativi. È intanto possibile sintetizzare ottant'anni di studi valutativi in una 
definizione di valutazione educativa come processo con tre prerogative ben distinguibili. Essa è (I) un giudizio di valore, emesso sulla (II) distanza rilevata tra una situazione auspicata e una effettivamente riscontrata e finalizzato (III) all'assunzione di decisioni volte alla riduzione di tale distanza.

Il giudizio di valore che caratterizza l'atto valutativo è relativo a una situazione percepita come problematica e ha la funzione di fornire un contributo alla sua risoluzione. Come verrà mostrato i tre elementi individuati nella definizione sopra riportata, già impliciti nella "Teoria della Valutazione" delineata da Dewey nel 1939, attestano l'imprescindibilità della consapevolezza e della partecipazione attiva degli attori coinvolti nel processo. Inoltre l'accertamento-misurazione deve porsi in rapporto dialettico con l'attribuzione di un giudizio di valore. A sua volta, il giudizio di valore va rapportato alla finalità del processo stesso, ovvero alla necessità di incidere sui processi decisionali.

La prima prerogativa, la valutazione come giudizio che determina un valore, rivela come la valutazione abbia una insopprimibile dimensione soggettiva. Questa dimensione esercita un duplice ruolo. Essa da un lato è fonte di elementi distorsivi, i cui effetti hanno costituito un oggetto privilegiato di studio per la docimologia. Ma la dimensione soggettiva è anche garante dell'autenticità dell'intero processo e delle sue possibilità di successo. È in base alla nostra prospettiva che stabiliamo che la situazione attuale è suscettibile di miglioramento e, dunque, di indagine valutativa. Soggettività non significa arbitrio: la condivisione di responsabilità valutative è, come mostrato nella prima parte di questo contributo, essenziale alla buona riuscita del processo stesso. La cultura della valutazione che si è affermata si percepisce invece come oggettiva, fondata sul numero e incentrata sulla classificazione di meriti e colpe. Spesso si parla di "fotografia del presente", una metafora abusata e del tutto ingiustificata: una fotografia dipende da inquadratura, focale, tempi di scatto, sensibilità iso. Non esiste una fotografia oggettiva.

Prendendo in considerazione la seconda delle tre prerogative menzionate, ovvero la necessità del giudizio valutativo di esprimersi sulla distanza rilevata tra una situazione auspicata e una effettivamente riscontrata, è possibile esplicitare in maniera più dettagliata il ruolo della misura nel processo valutativo. Per Dewey $(1939$, p. 37) «vi è sempre qualche osservazione del risultato ottenuto in paragone e in contrasto con quello atteso, tale che il paragone getti luce sulla effettiva adeguatezza delle cose adoperate come mezzi». Le misurazioni devono possedere «capacità operativa e mediatrice 
nella determinazione del giudizio finale» (Dewey, 1938). Quanto più sarà resa esplicita questa distanza tanto più la nostra azione sarà informata e avrà maggiori possibilità di successo. La misurazione è qui latamente intesa come processo che consente di collegare concetti astratti a indicatori empirici (Lucisano, 1989). Ogni operazione di raffronto, a prescindere dalla sua formulazione (numerica o non), ha una natura quantitativa: «il semplice confronto tra una qualità scadente e un'altra che viene considerata migliore non fa altro che proporre una graduatoria» (Gattullo, 1989, p. 158). Ma se la misurazione ha un ruolo rilevante (sebbene non egemonico) all'interno del processo valutativo, altra questione è il ricorso a una sua espressione numerica. Esso non è sempre indispensabile, talvolta è persino inappropriato: «è assurdo insistere sulla misurazione numerica quando il fine a cui la proposizione quantitativa si connette come mezzo a conseguenza è qualitativo, come è assurdo accontentarsi di una misurazione qualitativa (che in tal caso sarebbe un lavoro congetturale) nel caso di altre finalità. È in ultima analisi la natura del problema trattato che decide che genere di comparazione-misurazione sia richiesta al fine di ottenere una determinata soluzione. Alcuni si lamentano della riduzione a termini numerici, altri tutto vorrebbero ridurre a numero [...] gli uni e gli altri perdono di vista il significato logico della misurazione, che è determinato dal riferimento strumentale delle proposizioni quantitative ad una conseguenza intenzionale oggettiva. Gli uni e gli altri considerano le proposizioni come definitive e compiute, quando in realtà sono intermedie e strumentali» (Dewey, 1938, p. 260).

Riguardo a questo secondo aspetto l'attuale cultura della valutazione segna il passo sotto due punti di vista. In primo luogo fondandosi su un ricorso pervasivo all'espressione numerica, garante di oggettività e affidabilità. In secondo luogo, a differenza di quanto raccomandato da Ames, le misure prese fanno riferimento a strumenti di tipo normativo (consentono cioè solo un confronto con il posizionamento rispetto a popolazioni e contesti di riferimento, cfr. Corsini e Zanazzi, 2015) piuttosto che criteriale (incentrati su espliciti livelli di eccellenza o accettabilità analiticamente descritti).

Il terzo aspetto è relativo al perché della valutazione ed è quello più importante. Una valutazione che non è percepita come processo autenticamente finalizzato allo sviluppo di soggetti e contesti chiamati in causa nel giudizio ha notevoli probabilità di venire vissuta come mero assolvimento di un compito burocratico o come strumento di erogazione di premi o punizioni (Lucisano e Corsini, 2015; Corsini, 2015). Da questo punto di vista, le indagini sulle concezioni della valutazione da parte dei docenti risultano particolarmente significative. Si tratta di un filone di indagine relativamente recente 
(Brown, 2004, 2008, 2011; Davis e Neizel, 2011), che identifica quattro concezioni valutative dei docenti, le prime tre incentrate sulla base di finalità attribuite alla valutazione (miglioramento di insegnamento e apprendimento, accountability degli studenti; accountability delle scuole) e un'ultima, corrispondente a una visione della valutazione come processo inadeguato, inaccurato o irrilevante. Va segnalato come solo la prima, che comporta un attivo coinvolgimento di studentesse e studenti nel processo valutativo, abbia evidenziato associazioni positive con i livelli di apprendimento (Hattie e Timperley, 2007).

\section{Per concludere: la necessità di un'altra narrazione valu- tativa}

Secondo Greenstein (2010), a rendere efficace la valutazione non è l'impiego di certi strumenti, ma l'uso delle informazioni raccolte. Se queste vengono usate per trasformare la didattica, allora la valutazione avrà modo di incidere positivamente sull'apprendimento, altrimenti avremo una mera raccolta di dati impiegabile con finalità rendicontative. Come accennato, oggi, mezzo secolo dopo Scriven e venti anni dopo il lavoro di Black e Wiliam, tutto sommato siamo ancora lì: sappiamo che la valutazione formativa funziona, ma è praticata in misura ridotta nelle scuole e nelle accademie (sebbene sappiamo che funzioni anche in università, cfr. Coggi e Pizzorno, 2018). Perché? Per capire il perché è utile tornare ancora a Dewey. Nella sua "Teoria della valutazione", il filosofo spiega chiaramente che il tempo di appropriate valutazioni è il futuro, dato che esse «non vertono su cose od eventi occorsi o che già esistono, ma riguardano cose che ancora devono esser poste in essere». In pratica, una valutazione è efficace e autentica se ci lega a un impegno nel futuro, e questo equivale a dire che chi è impegnato nella valutazione, tanto come valutatore quanto come valutato, deve essere disposto a modificare la propria azione in seguito ai giudizi di valore emessi sulle misure raccolte. Ma quanti docenti sono disposti a fare questo? Quanti, piuttosto che aprire la propria routine all'incertezza di un cambiamento coerente con i giudizi di valore espressi, preferiscono separare nettamente valori e prassi, finendo col separare valutazione e insegnamento?

Se, come esplicitato in apertura, la narrazione in educazione consente di conferire senso all'esperienza pregressa e presente per orientare quella futura, un approccio valutativo utile a una didattica incentrata su prassi narrative non può che farsi carico di rivolgersi a un passato ricco di stimoli al fine di intraprendere una scelta netta tra culture valutative inconciliabili. Senza 
dimenticare che, come ammoniva Cronbach (1980), mentre l'accountability implica il guardarsi indietro per distribuire meriti o colpe, la valutazione serve per capire eventi e processi e indirizzare attività future.

\section{Riferimenti bibliografici}

Ames C. (1992), "Classroom: Goals, structures and student motivation”, Journal of Educational Psychology, 84: 261-271.

American Statistical Association (Asa) (2014), ASA statement on using value-added models for educational assessment, Alexandria.

Amrein-Beardsley A. (2014), Rethinking Value-Added Models in Education, Routledge, New York.

Baker E.L., Barton P.E., Darling-Hammond L., Haertel R., Ladd H.F., Linn R., Ravitch D., Rothstein R., Shavelson R.J. and Shepard L.A., (2010), Problems with the use of student test scores to evaluate teachers, Economic Policy Institute, Washington.

Batini F., Bartolucci M. e De Carlo E. (2017), "Fight Dispersion Through Education: The Results of the First Cycle of the NoOut Project", Mind, Brain, and Education, 11, 4: 201212.

Batini F. e Corsini C. (2016), Insegnare e valutare per competenze, in Batini F. Insegnare e valutare per competenze, pp. 79-98, Loescher, Torino.

Batini F. e Del Sarto G. (2007), Raccontare storie. Politiche del lavoro ed orientamento narrativo, Carocci, Roma.

Black P. and Wiliam D. (1998), "Inside the black box: Raising standards through classroom assessment”, Phi Delta Kappan, 80, 2: 139-148.

Black P., Harrison C., Lee C., Marshall B. and Wiliam D. (2004,), "Working inside the black box: Assessment for learning in the classroom", Phi Delta Kappan, 86, 1: 8-21.

Borrelli D. (2015), Contro l'ideologia della valutazione. L'ANVUR e l'arte della rottamazione dell'università, Jouvence, Sesto San Giovanni.

Brown G.T.L. (2004), “Teachers' conceptions of assessment: implications for policy and professional development", Assessment in Education: Principles, Policy \& Practice, 11, 2: 301-318

Brown G.T.L. (2008), Conceptions of assessment: Understanding what assessment means to teachers and students, Nova Science Publishers, New York, NY.

Brown G.T.L. (2011), “Teachers' conceptions of assessment: Comparing primary and secondary teachers in New Zealand", Assessment Matters, 3: 45-65.

Coggi C. e Pizzorno M.C. (2018), La valutazione formativa in università. Trasparente, condivisa, regolativa, sostenibile, in Notti A.M., a cura di, La funzione educativa della valutazione, pp. 37-58, Pensa, Lecce.

Corsini C. (2009), Il valore aggiunto in educazione, Nuova Cultura, Roma.

Corsini C. (2012), "La lezione americana: l'impiego del Valore Aggiunto nella valutazione di scuole e insegnanti”, Scuola Democratica, 6: 60-75.

Corsini C. (2015), Valutare scuole e docenti. Un'indagine sul punto di vista di chi insegna, Edizioni Nuova Cultura, Roma.

Corsini C. e Zanazzi S. (2015), "Valutare scuola e università: approccio emergente, interventi e criticità", I problemi della pedagogia, 2: 305-334.

Cronbach L.J. (1980), Our Ninety-five thesis, in Cronbach, ed., Toward Re-form of Program Evaluation, pp. 25-34, Jossey-Bass, San Francisco. 
Davis D.S. and Neitzel C. (2011), "A self-regulated learning perspective on middle grades classroom assessment", Journal of Educational Research, 104: 202-215.

Dewey J. (1938), Logic, the Theory of Inquiry, Holt and Co, New York, (trad. it. Logica, teoria dell'indagine, Einaudi, Torino, 1949).

Dewey J. (1939), Theory of Valuation, University press, Chicago (trad. it. Teoria della valutazione, La Nuova Italia, Firenze, 1960).

Earl L.M. (2013), Assessment as Learning: Using Classroom Assessment to Maximize Student Learning Earl, Corwin Press, Thousand Oaks.

Elliott E.S. and Dweck C.S. (1988), "Goals - an approach to motivation and achievement", Jnl of Personality \& Social Psych, 54: 5-12.

Fitz-Gibbon C.T. and Kochan S. (2000), School Effectiveness and Education Indicators, in Teddlie C. and Reynolds D., eds., The International Handbook of School Effectiveness Research, pp. 257-280, Falmer, Londra-New York.

Fondazione Giovanni Agnelli (2014), La valutazione della scuola, Laterza, Roma.

Foucault M. (2014), Subjectivité et vérité. Cours au Collège de France 1980-81, édition établie sous la direction de François Ewald et Alessandro Fontana par Frédéric Gros, Seuil/Gallimard, Paris (trad. it Soggettività e verità, Feltrinelli, Milano, 2017).

Gattullo M. (1989), "Quantitativo e qualitativo in educazione e nella ricerca educativa", Scuola e Città, 4: 158-162.

Greenstein L. (2010), What Teachers Really Need to Know About Formative Assessment, Association for Supervision \& Curriculum Development, Alexandria.

Hattie J. and Timperley H. (2007), "The power of feedback", Review of Educational Research, 77, 1: 81-112.

INVALSI (2008), La ricerca IEA-PIRLS 2006, FrancoAngeli, Roma.

Koretz D. (2009), Measuring Up: What Educational Testing Really Tells Us, University press, Harvard.

Kuhn T. (1962), The Structure of Scientific Revolutions, University of Chicago Press, trad. it. La struttura delle rivoluzioni scientifiche, Torino, Einaudi, 1978.

Lucisano P. (1989), Misurare perché e come, in P. Lucisano, a cura di, Lettura e comprensione, pp. 43-68, Loescher, Torino.

Lucisano P. (2011), "La sindrome del figlio dell'uomo", in Journal of Educational, Cultural and Psychological Studies, 1: 155-167.

Lucisano P. (2015), “Comprendere la valutazione”, I problemi della pedagogia, 2: 335-343.

Lucisano P. e Corsini C. (2015), "Docenti e valutazione di scuole e insegnanti”, Giornale Italiano della ricerca educativa - Italian Journal of Educational Research, 15: 97-109.

Pinto V. (2012), Valutare e punire, Cronopio, Napoli.

Pinto V. (2014), "Un nuovo immaginario: l'amministrazione dell'evidenza", Imago, III, 4: 722.

Ravitch D. (2010), The Death and Life of the Great American School System: How Testing and Choice Are Undermining Education, Basic Books, New York.

Scriven M. (1967), The metodology of evaluation, in Tyler R.W., Gagne R.M. and Scriven M., eds., Perspectives of curriculum evaluation, Vol. I, pp. 39-83, Rand McNally, Chicago.

Stiggins R.J. (2002), “Assessment crisis: The absence of assessment for learning”, Phi Delta Kappan, 83, 10: 758-765.

Swaffield S. (2011), "Getting to the heart of authentic Assessment for Learnig", Assessment in Education: Principles, Policy and \& Practice, 18, 4: 443-449.

Trinchero R. (2018), Attivare cognitivamente con la valutazione formante, in Notti A.M., a cura di, La funzione educativa della valutazione, pp. 73-90, Pensa, Lecce.

Veggetti M.S. (2016), Narrazione e educazione, tra dimensione storico-culturale e apprendimento cooperativo, in Sposetti P. e Szpunar G., a cura di, Narrazione e educazione, Nuova 
Cultura, Roma.

Visalberghi A. (1955), Misurazione e valutazione nel processo formativo, Edizioni di Comunità, Milano.

Watkins C. (2001), Learning about Learning enhances performance, Institute of Education, University of London, London.

Wiggins G. (1993), Assessing Student Performance: Exploring the Purpose and Limits of Testing, Jossey-Bass, San Francisco.

Wiggins G. (1998), Educative Assessment: Designing Assessments to Inform and Improve Student Performance, Jossey-Bass, San Francisco. 


\title{
Le nuove tecnologie per la prevenzione della dispersione scolastica: le possibilità offerte dal digitale alla lettura
}

\author{
di Floriana Falcinelli
}

Il problema della dispersione scolastica è da molti anni all'attenzione dei ricercatori tanto che recenti indagini condotte nelle scuole, dando voce proprio agli studenti, ci fanno capire l'ampiezza e la complessità del fenomeno (Batini e Bartolucci, 2016).

Anche il MIUR negli annuali rapporti a cura del Servizio Statistico sulla dispersione scolastica, sottolinea la necessità di monitorare con attenzione il fenomeno, che evidenzia uno stato di malessere a cui la scuola deve saper rispondere, puntando sull'innovazione didattica e su scelte strategiche che sappiano riconoscere e valorizzare il potenziale degli studenti (Rossi Doria e Tabarelli, 2016).

Da tempo l'introduzione delle tecnologie nella pratica didattica è associata al concetto di innovazione anche se è noto che l'uso dello strumento non è sufficiente da solo a garantire un reale cambiamento nel processo di insegnamento/apprendimento, tale da risultare efficace a promuovere il diritto allo studio di tutti, in una logica autenticamente inclusiva.

D'altra parte le tecnologie sono state interpretate più come strumenti compensativi che come risorse emancipative e di sviluppo degli apprendimenti.

Le Linee guida per il diritto allo studio degli alunni e degli studenti con DSA, allegate al D.M. 12 luglio 2011, ribadiscono quanto affermato nella legge 170/2010 nella quale si richiamavano le istituzioni scolastiche a garantire l'introduzione di strumenti compensativi, compresi i mezzi di apprendimento alternativi e le tecnologie informatiche, come strumenti didattici e tecnologici che sostituiscono o facilitano la prestazione richiesta nell'abilità deficitaria.

Eppure dovremmo cercare di uscire da questa logica, importante, ma sicuramente riduttiva, per vedere come le tecnologie possano amplificare le capacità, le potenzialità presenti in ogni soggetto e permettere nuovi processi 
di acquisizione della cultura e di comunicazione, con linguaggi e strategie diversi, ma non per questo meno importanti dal punto di vista educativo.

Le tecnologie possono dunque essere considerate strumenti di mediazione sia nei processi di appropriazione della cultura che nei processi di crescita personale, per cui possono essere utilizzate per: ridurre le difficoltà di apprendimento, facilitare e promuovere la capacità di rapportarsi e di comunicare con la realtà sociale, scolastica e lavorativa, lo sviluppo e l'acquisizione della conoscenza, lo sviluppo e l'acquisizione di nuove potenzialità espressive e creative, l'autonomia e il senso di autostima.

Eppure tutto quello che abbiamo detto finora sull'importanza delle tecnologie non deve farci perdere di vista che possono esserci degli aspetti problematici che impongono una riflessione attenta.

È stato messo in luce che un uso intenso ed esclusivo di tecnologie digitali, peraltro con costante connessione alla rete, può favorire la tendenza ad impoverire la sfera della comunicazione interpersonale, delle autonomie personali, delle autonomie nei movimenti. Si potrebbe inoltre correre il rischio di ridurre la capacità dei ragazzi di esplorare e di interagire con gli oggetti, utilizzando il tatto, l'udito, il senso propriocettivo e cinestesico e la capacità di orientarsi nello spazio con il proprio corpo. Così come potrebbe emergere la tendenza a ridurre la complessità del sapere, impoverendola e riducendola a frammento (Ranieri, 2006).

Proprio per questo l'uso delle tecnologie va sempre sostenuto da alcuni principi di fondo che si ispirano ad una logica blended, di integrazione tra $\mathrm{i}$ diversi mediatori didattici: occorre prima di tutto favorire la continuità e l'integrazione tra l'uso di strumenti ad elevata tecnologia e dei programmi relativi e l'uso delle tecnologie tradizionali, basate su una interazione più diretta tra educatore e ragazzo e del ragazzo con l'esperienza diretta con la realtà; è necessario salvaguardare la continuità ed integrazione nel vissuto del ragazzo tra esperienza mediata da strumenti tecnologici con l'esperienza diretta con le cose e sulle cose, con le persone e nel gruppo reale; occorre valorizzare la possibilità data da molti applicativi di personalizzare e programmare le risposte, favorendo lo sviluppo di fondamentali abilità cognitive e metacognitive e la consapevolezza delle proprie strategie di pensiero; è opportuno sostenere la possibilità di apprendere secondo ritmi e percorsi individualizzati, sostenendo motivazione e interesse nonché la capacità di imparare ad apprendere, di organizzare, strutturare e costruire informazioni in modo più personale; bisogna evitare che imparare a servirsi delle possibilità offerte dalle tecnologie diventi un fatto puramente meccanico e nozionistico, che poco giova alla maturazione e alla crescita globale del soggetto; occorre 
piuttosto progettare percorsi educativi in cui le tecnologie siano un'opportunità per affinare le capacità espressive, creative, immaginative e di astrazione.

Perciò occorre una costante osservazione degli studenti e una reale flessibilità nell'adattare le diverse soluzioni con opportuna gradualità e intercambiandole a seconda del livello di maturazione, degli obiettivi perseguiti o che si vuole perseguire, della motivazione dei ragazzi.

Per questo è necessario inserire l'uso delle tecnologie in uno sfondo connettivo, che permetta la costruzione di un ambiente di apprendimento con significati condivisi tra insegnanti, studenti ma anche genitori.

In questo senso il lavoro con le tecnologie diventa occasione di scambio, di condivisione, di costruzione collaborativa in cui tutti possono apportare un loro contributo, in un ambiente di apprendimento progettato nella logica dell'eSchooling.

Per eSchooling si intende un modello integrato di didattica digitale che si pone l'obiettivo di attuare modalità educative innovative che sfruttino le potenzialità offerte dalle ICT (Information Communication Technologies) di personalizzazione dell'apprendimento e di coinvolgimento di tutti gli attori (docenti, discenti genitori, comunità locali, gruppi specifici) al processo didattico. L'ipotesi è quella di realizzare una classe "full digital" in cui lo spazio fisico è integrato a uno o più spazi virtuali collegati tra loro nel quale è possibile costruire un ponte, bridging, tra apprendimento formale, scolastico e l'apprendimento informale che è prodotto sempre più dalla diffusa esperienza con le nuove tecnologie.

L'uso delle ICT consente così la piena realizzazione di un ambiente di apprendimento in cui è allestito un variegato repertorio di risorse, di tecniche appropriate, di strategie e strumenti di scaffolding per permettere un apprendimento significativo, creativo, emancipante di tutti gli allievi, in prospettiva pienamente inclusiva.

Le attività didattiche che possono essere proposte sono molteplici e accompagnano tutte le azioni didattiche che prevedono momenti espositivi in cui è possibile presentare o esporre un contenuto di studio alla classe intera, integrati da momenti in cui è possibile attivare, organizzando gli studenti in gruppi, percorsi di ricerca, esplorazione di materiali diversi anche multimediali, o la costruzione collaborativa, mediante esperienze di negoziazione e di discussione, di prodotti che possano esprimere la capacità creativa degli studenti e che possano poi essere condivisi e socializzati per sperimentare il confronto tra punti di vista diversi.

Molte dunque le attività che si possono programmare: 
- attività didattiche con tutta la classe con il PC o con la LIM per comprendere, organizzare, memorizzare, rielaborare i contenuti privilegiando una modalità percettivo-iconica-emozionale;

- attività individuale $\mathrm{o}$ a piccolo gruppo con tablet e altri dispositivi mobili per produrre contenuti multimediali;

- attività individuale a casa (podcast, chiavette USB, dispositivi mobili) per approfondimento, consolidamento, rielaborazione e comunicazione;

- attività in rete, su ambienti virtuali, piattaforme di gestione (Edmodo, Moodle, social network per favorire la cooperazione tra pari, tra insegnanti, tra scuole, per costruire un ponte con le molteplici esperienze di apprendimento non formale nel WEB 2.0).

Il setting tecnologico dovrebbe prevedere l'integrazione di molteplici strumenti anzitutto la LIM (Lavagna Interattiva Multimediale) per la costruzione collaborativa di un compito, la correzione condivisa di un compito, la presentazione-discussione collettiva di un lavoro individuale o di gruppo, la raccolta di materiali, le risposte a domande ed esercizi, l'arricchimento della spiegazione (Faggioli, 2010, p. 79).

La LIM può essere usata in modo molto versatile dal docente: permette di svolgere lezioni più coinvolgenti e motivanti, ma dovrebbe sempre più diventare una superficie di interazione tra docente e allievi e tra gli allievi, spazio di costruzione condivisa della conoscenza, proporre contenuti aperti, accedere a realtà esterne alla classe, visualizzare e analizzare informazioni, documenti ed esperienze, realizzare forme di lavoro per gruppi, approcci euristici per scoperta guidata permette di valorizzare le $3 \mathrm{X}$ dei nativi digitali eXplore, eXchange, eXpress (Biondi, 2008). La LIM inoltre risulta particolarmente utile per i soggetti con difficoltà scolastiche di varia natura dal momento che facilita processi di scrittura e lettura e di comprensione di concetti matematici, in un contesto di classe in cui è possibile condividere con gli altri percorsi di apprendimento. La LIM è uno strumento intrinsecamente inclusivo perché consente: di utilizzare risorse multimediali che facilitano processi di intuizione mediante l'immersività e l'attivazione degli aspetti percettivo-emozionali; consente inoltre di trasformare, destrutturare, semplificare i materiali didattici, di gestire il tempo recuperando il lavoro già fatto, di creare percorsi personalizzati da gestire anche in modo collettivo.

In questo scenario gli insegnanti, in quanto soggetti ermeneutico-critici che cercano di attribuire senso al proprio agire didattico e al sistema complesso in cui esso avviene, debbono considerare le ICT come nuovi contesti/ambienti di insegnamento apprendimento, come risorse per l'azione formativa, cogliendo però gli elementi di diversità che essi introducono nella tradizionale relazione didattica: il processo di apprendimento si avvale di 
esperienze multidimensionali, diviene sempre più costruttivo e reticolare, condiviso socialmente, sperimenta le dimensioni del gioco, dell'immaginario, dell'espressività emozionale, è alimentato da eventi comunicativi informali (Ferri, 2013).

Il ruolo del docente dunque cambia profondamente: «Bisogna opporsi all'idea che grazie alle nuove tecnologie gli studenti possano fare da soli esse al contrario attribuiscono un ruolo ancora più importante all'insegnante che non è semplicemente mediatore di conoscenze già articolate, ma è coinvolto più profondamente nel supportare gli studenti nell'organizzazione del pensiero e nello sviluppo di nuovi tipi di competenze di cui avranno bisogno per comprendere i materiali digitali» (Laurillard, 2014, p. 13).

Come tutto questo impatta con la lettura, attività tradizionalmente molto sollecitata in ambito scolastico?

L'esperienza con le nuove tecnologie, in particolare quelle digitali e di rete, è un'esperienza assolutamente diffusa, generalizzata nei ragazzi: attraverso le nuove tecnologie essi conoscono il mondo, imparano a comunicare e a fare gruppo. Ma questo non vuol dire che pensare alle nuove tecnologie e pensarle come risorse preziose per accedere alla cultura e per comunicare, significhi contrapporre le tecnologie al libro.

Se pensiamo al motivo della loro rapida diffusione e del successo che hanno con i ragazzi, dobbiamo riconoscere che esse rappresentano tutto quello che caratterizza l'apprendimento cosiddetto informale, libero, creativo, divergente, connesso alla possibilità potenzialmente infinita di esplorare e scoprire cose nuove, cioè tutto ciò che si caratterizza come non-scuola. La scuola è infatti ancora librocentrica, alfabetica, orientata verso un sapere formalizzato e una produzione e riproduzione della cultura di natura prevalentemente convergente.

Un altro elemento molto importante su cui riflettere è che parliamo oggi sempre più di mobile devices, come di quelle tecnologie che permettono di fruire di tutti i tipi di informazione e di collegarsi in rete rapidamente, superando ogni vincolo di spazio e tempo, di diffondere e condividere le informazioni ma soprattutto di comunicare in modo diffuso e aperto, connettendosi, in ogni momento, da ogni parte, di costruire gruppi, tribù, comunità con cui condividere emozioni, interessi ed anche conoscenza.

Queste tecnologie ci hanno fatto capire che il sapere può essere accessibile per tutti e in modo rapidissimo. In rete tutto il sapere è disponibile, immediato, aggiornato mentre invece quello che propone la scuola appare ai ragazzi vecchio, statico, incentrato sul passato. L'informazione che si trova in rete è dinamica, moderna, vive della quotidianità, è solitamente multimediale, e ciò la rende più accattivante, coinvolgente e più fruibile da tutti. Un 
ragazzo può reperire in rete la musica preferita, ascoltarla, scaricarla, poi andarci in giro, così foto e video, spazio di scambio e di confronto con i compagni.

Certamente anche con le ICT si richiede un processo di lettura ma in modo molto diverso da quello richiesto dal libro: è una lettura per immersione, visuale, perché il supporto che rende fruibile l'informazione è un video. Procede per intuizione, in modo globale e sincretico, per progressivi insight; è quindi molto lontana dalla modalità analitica, lineare sequenziale che è tipica della lettura richiesta da un libro alfabetico.

Un altro aspetto particolarmente importante è che l'informazione offerta dalle ICT è ipertestuale: vuol dire che da una pagina, "cliccando" su alcune parole definite "calde" si possono esplorare nuove pagine collegate alla principale da nessi logici; chi è abituato ad utilizzare la rete apre una pagina, poi esplora le altre concatenate in modo reticolare; si costruisce a livello mentale un'associazione, con legami tra le informazioni talvolta più o meno confusi, ma aperti a nuovi nessi che sono offerti dall'esplorazione delle pagine successive.

In questo sistema caratterizzato da interessanti ibridazioni tecnologiche $\mathrm{e}$ linguistiche, c'è ancora spazio per il libro? La domanda è mal posta, poiché è assolutamente sbagliato considerare il libro in alternativa a tutto ciò; il libro va visto dentro il sistema come qualcosa di integrato in esso, pur mantenendo una sua specificità. Così come hanno una loro specificità la radio, la televisione, la stampa che non sono state certamente annullate dal computer e dalla rete, ma hanno iniziato a ridefinire le proprie caratteristiche e ad assumere il codice digitale come sfida per l'innovazione.

In questa prospettiva il libro potrà diventare e-book, integrato ma diverso da quello alfabetico, fisico, di cui si fa esperienza con tutti i sensi; l'e-book è un prodotto multimediale che può espandersi grazie al collegamento con le risorse di rete, può essere esplorato in modo personalizzato, commentato in spazi virtuali sociali, in cui si condivide l'esperienza di lettura.

Gli e-book sono tool attraverso i quali è possibile caricare e leggere contenuti digitali, in numero quantitativamente elevato rispetto alle possibilità offerte dall'editoria classica (Roncaglia, 2010); attraverso devices dedicati gli e-readers attualmente dotati della tecnologia e-ink ovvero l'inchiostro digitale che non affatica la vista, o generalisti come l'iPad, oggi è possibile avere a disposizione dovunque un considerevole numero di testi, a basso costo.

Di questi problemi si parla anche nel PNSD (MIUR, 2015) che individua tre azioni, integrate tra loro, sui nuovi formati digitali sui prodotti per la di- 
dattica e sul rinnovato ruolo delle Biblioteche scolastiche: 1'Azione \#22: prevede Standard minimi e interoperabilità degli ambienti on line per la didattica, l'Azione \#23: Promozione delle Risorse Educative Aperte (OER) e linee guida su autoproduzione dei contenuti didattici e l'Azione \#24: Biblioteche Scolastiche come ambienti di alfabetizzazione all'uso delle risorse informative digitali.

In particolare per questa ultima azione si dice: «un potenziamento e un aggiornamento della missione delle biblioteche scolastiche che in molte realtà faticano a trovare spazio mentre in altre svolgono un ruolo determinante per l'attività di promozione della lettura anche grazie all'uso della rete e di strumenti digitali [...]. Occorre cogliere l'opportunità dell'integrazione tra mondo della lettura e della scrittura ed esperienze digitali».

Le potenzialità didattiche dei libri digitali sono interessanti purché non ci si limiti alla lettura elettronica di PDF, ma ci si muova nella logica del "testo espanso" (Darnton, 2009). Sarebbe auspicabile che la possibilità di connettersi in rete apra la lettura a inedite modalità di condivisione, che il PDF possa prevedere link che esemplifichino delle astrazioni con l'impiego di linguaggi multimediali tali da consentire una ibridazione tra lineare e reticolare, che si possano aggiungere note a margine al testo e farle apparire nel device privato di persone che appartengono alla cerchia di amici, creando delle vere e proprie comunità di apprendimento. Si potrebbe infine arrivare all'idea della creazione di libri digitali aperti, costruiti dai ragazzi e dai docenti attraverso percorsi di ricerca.

Si potrebbe così sviluppare nei ragazzi l'esperienza di essere autore utilizzando un normale programma di videoscrittura ma anche un social software come Wiki che permette la costruzione collaborativa di un testo scritto, come ben dimostrato dalla realtà di Wikipedia, oppure invitare i ragazzi a costruirsi un blog personale e a fare rete con i compagni nei social network.

E ciò che conta è proprio stimolare e mantenere nei ragazzi la voglia di scoprire, di conoscere, qualunque sia il supporto che lo consente. Questo è la base per rimotivare gli studenti alla scuola e permettere a tutti di raggiungere il successo formativo, contribuendo così a combattere di fatto le problematiche che possono portare alla dispersione scolastica.

\section{Riferimenti bibliografici}

Batini F. e Bartolucci M., a cura di (2016), Dispersione scolastica. Ascoltare i protagonisti per comprenderla e prevenirla, FrancoAngeli, Milano.

Biondi G., a cura di (2008), LIM. A scuola con la lavagna interattiva multimediale - Nuovi linguaggi per innovare la didattica, Giunti, Firenze. 
Darnton R. (2009), The case for books. Past, present and future, Public Affairs, New York. Faggioli M. (2010), Tecnologie per la didattica, Apogeo, Milano.

Ferri P. (2013), La scuola 2.0. Verso una didattica aumentata dalle tecnologie, Spaggiari, Parma.

Laurillard D. (2014), Insegnamento come scienza della progettazione. Costruire modelli per apprendere con le tecnologie, FrancoAngeli, Milano.

MIUR (2015), Piano Nazionale Scuola Digitale, tetso disponibile al sito: http://www.istruzione.it/scuola_digitale/allegati/2014_archivio/home03_140601_Piano $\% 20$ Nazionale $\% 20$ Scuola\%20Digitale.pdf.

Ranieri M. (2006), Formazione e cyberspazio. Divari e opportunità nel mondo della rete, Edizioni ETS, Pisa.

Roncaglia G. (2010), La quarta rivoluzione. Sei lezioni sul futuro del libro, Laterza, Bari.

Rossi Doria M. e Tabarelli S., a cura di (2016), Reti contro la dispersione scolastica. I cantieri del possibile, Erickson, Trento. 


\title{
Insegnanti abili ed efficaci: una risorsa per contrastare l'abbandono scolastico
}

\author{
di Mina De Santis, Annalisa Morganti ${ }^{1}$
}

\section{Introduzione}

Questo contributo nasce dal lavoro condotto congiuntamente all'interno degli insegnamenti di Didattica Generale e Pedagogia Speciale svolti presso il Corso di Studi in Scienze della Formazione Primaria dell'Università di Perugia e dedicati alla formazione dei nuovi e futuri insegnanti, all'interno di una prospettiva educativa inclusiva e consapevole dell'importanza del coinvolgimento degli studenti, in tutti gli ordini e gradi del sistema di istruzione.

Il nostro intento è di evidenziare conoscenze, abilità e competenze appartenenti al profilo professionale dell'insegnante - qualsiasi sia l'ordine o grado scolastico di appartenenza -, capace di pensare, progettare e realizzare una didattica che sin da principio è attenta alle esigenze di ciascuno dei suoi studenti, senza che nessuno si "perda" nei vari segmenti del percorso formativo.

La didattica inclusiva, che si qualifica come una didattica di qualità per tutti, non è una corsia d'accesso privilegiata solo per allievi con disabilità o che presentano disagi, disturbi o difficoltà educative di varia natura, ma si connota sempre più come uno stile d'insegnamento, un orientamento educativo e didattico quotidiano che si prefigge di rispettare e valorizzare le differenze presenti in tutti gli allievi (Morganti e Bocci, 2017; Morganti e Signorelli, 2017).

Si parla di diversità in educazione, come ricorda Mitchell (2016), in termini di abilità, lingua, genere, etnia, religione, status sociale, culturale o economico e il compito prioritario degli insegnanti è di evitare che tali differenze

${ }^{1}$ È da attribuire ad Annalisa Morganti la scrittura del paragrafo 2, a Mina De Santis quella del paragrafo 3. L'introduzione è scritta da entrambe le autrici del testo. 
si trasformino per gli allievi in consistenti barriere all'apprendimento e alla partecipazione alla vita scolastica e sociale.

In questo capitolo soffermeremo la nostra attenzione su due dimensioni che riteniamo essenziali e non accessorie alla costruzione del profilo professionale dell'insegnante: quella delle abilità personali e quella delle competenze metodologico-didattiche.

Sul piano personale presenteremo alcuni studi che mostrano l'importanza delle abilità sociali ed emotive degli insegnanti ai fini della costruzione di un contesto di apprendimento positivo e accogliente per tutti, troppo spesso considerate caratteristiche individuali di cui, sin dalla nascita si è provvisti o sprovvisti. Sul piano metodologico-didattico porremo l'attenzione su quelle modalità didattiche "attive" che sollecitano e promuovono l'interesse, la motivazione e l'impegno degli studenti verso l'apprendimento.

Entrambi questi aspetti invitano ad una riflessione non solo culturale, ma che investe anche il piano delle pratiche didattiche e delle politiche educative con percorsi formativi maggiormente orientati verso tali traguardi.

\section{Creare contesti di apprendimento efficaci: il ruolo delle abilità personali degli insegnanti}

Numerose sono le ricerche che si sono interessate di studiare le caratteristiche principali e ricorrenti di un insegnante "efficace", ovvero capace di avere un "impatto" visibile, misurabile e reale sul progresso dei propri studenti ${ }^{2}$. Come ricorda Hattie, nella sua intervista all'edizione italiana del volume Visible learning for teachers. Maximizing impact on learning (2012) ${ }^{3}$, gli insegnanti devono osservare l'impatto del loro insegnamento attraverso gli occhi degli studenti e condividere cosa può rappresentare questo "im-

\footnotetext{
${ }^{2}$ Con il termine "efficacia", si fa riferimento alla differenza nei risultati (non solo scolastici) ottenuti da un gruppo sperimentale, che effettua un tipo di intervento da indagare (ad esempio l'uso di una determinata strategia didattica) e un gruppo controllo (che non effettua alcun intervento). La differenza nei risultati ottenuti dai gruppi determina un valore statistico chiamato Effect Size (ampiezza dell'effetto) che consente di esprimere quanto un intervento è stato efficace, ovvero, ha prodotto i risultati sperati, in una popolazione controllata di studenti. Per approfondimenti sul tema dell'Evidence-based Education si veda: Calvani, 2011a, 2012, 2014; Vivanet, 2013, 2014; Morganti, 2012; Cottini e Morganti, 2015, 2016.

${ }^{3}$ Il testo, curato da G. Vivanet, è stato tradotto in italiano dalle edizioni Erickson con il titolo: Apprendimento visibile, insegnamento efficace (2016). Il testo contiene una interessante intervista del curatore all'autore sui principali messaggi veicolati dal testo.
} 
patto" nella scuola. «È un miglioramento dei risultati, sono punteggi più elevati ai test, o comprende anche l'amore per l'apprendimento, il desiderio di reinvestire nella formazione, una sensazione di competenza, il piacere della collaborazione, una sensibilità sociale per lavorare con gli altri, il rispetto per se stessi e quello per gli altri-o tutte queste cose insieme?» (Hattie, 2016, p. 23).

La risposta non può che trovarsi nella somma di tutti questi elementi, ciascuno dei quali concorre al progresso e alla crescita personale, sociale e formativa dello studente.

Obiettivi educativi e formativi ambiziosi, che richiedono, per essere raggiunti, una professionalità elevata capace di coniugare una serie articolata di conoscenze, abilità, valori, capacità riflessive ed autoriflessive.

Rubio (2009) in un'interessante rassegna di studi articola le principali caratteristiche di un insegnante efficace presentando da un lato, le sue abilità professionali dall'altro, quelle personali (tab. 1).

Tab. 1 - Caratteristiche personali e professionali dell'insegnante "efficace" (adattato da Rubio, 2009)

\begin{tabular}{|l|l|}
\hline \multicolumn{2}{|c|}{ PROFILO DELL'INSEGNANTE EFFICACE } \\
\hline $\begin{array}{l}\text { ABILITA் } \\
\text { PROFESSIONALI }\end{array}$ & Conoscenze disciplinari \\
\cline { 2 - 2 } & Capacità di progettazione \\
\cline { 2 - 2 } & Gestione della classe e organizzazione \\
\cline { 2 - 2 } & Gestione dei comportamenti in classe \\
\cline { 2 - 2 } & $\begin{array}{l}\text { Rispondere alle differenze individuali e ai diversi biso- } \\
\text { gni educativi }\end{array}$ \\
\cline { 2 - 2 } & Competenze comunicative \\
\cline { 2 - 2 } & Avere fiducia in se stessi e nel proprio insegnamento \\
\cline { 2 - 2 } & Motivare all'apprendimento \\
\cline { 2 - 2 } & Rispettare, essere imparziali ed equi \\
\cline { 2 - 2 } & Avere aspettative positive verso se stessi e gli studenti \\
\hline ABILITÀ & Aver cura dei propri allievi \\
\cline { 2 - 2 } PERSONALI & Conoscere gli studenti individualmente \\
\cline { 2 - 2 } & Stabilire relazioni positive con lo studente \\
\cline { 2 - 2 } & Costruire un positivo clima di classe \\
\hline
\end{tabular}

È chiaro, anche da tale articolazione, il ruolo che hanno abilità e tratti personali del docente, nel contribuire all'efficacia dell'attività didattica. Anche Hattie (2012) conferma il peso di questi aspetti ponendo, ad esempio, le relazioni insegnante-studenti tra le influenze che hanno un impatto "alto" (E.S 0.72) sui processi di apprendimento. 
Come ricorda Concina (2016), di là delle caratteristiche specifiche di ogni singola disciplina insegnata, i docenti "efficaci" manifestano capacità sviluppate di stimolare e supportare il processo di apprendimento negli studenti, instaurando con loro relazioni interpersonali significative per entrambi.

Anche Catalano et al. (2014) dalla loro indagine sulle pratiche di insegnamento efficaci sui docenti italiani di scuola dell'infanzia e primaria fanno emergere alcune dimensioni prevalenti di un insegnamento efficace che riguardano le capacità comunicative, l'uso di metodologie attive - di cui parleremo in seguito - e l'attenzione, anche in questo caso, alle relazioni interpersonali. Relazioni inadeguate con gli insegnanti possono accompagnare paura e allontanamento degli studenti dalla scuola e, nel tempo, generare sentimenti di alienazione, disimpegno e nei casi più estremi abbandono precoce.

Come ricordano Jennings e Greenberg (2009), quando gli studenti si sentono alienati da scuola, corrono un rischio maggiore di sviluppare comportamenti antisociali, delinquenza e fallimenti accademici. Al contrario, relazioni di sostegno con gli insegnanti possono promuovere sentimenti di sicurezza e connessione tra gli studenti, fornendo il supporto sociale necessario per crescere socialmente, emotivamente e scolasticamente.

Sebbene ciò sia vero per gli allievi in tutti i gradi scolastici, è particolarmente decisivo nei primi anni di scolarizzazione, poiché le prime esperienze di un bambino con i suoi insegnanti possono influenzare pesantemente le relazioni future con altri e con la scuola più in generale.

I ristretti ambiti e le finalità del presente lavoro non consentono un'analisi dettagliata di tutte le posizioni teoriche che possono essere raccolte a sostegno dell'importanza delle dimensioni personali per un insegnamento efficace, qui mi limiterò pertanto, a fare delle brevi considerazioni conclusive, lasciando al lettore altre possibilità di ampliare questo aspetto.

La breve carrellata di ricerche presentate evidenzia importanti ripercussioni sul piano della formazione iniziale e in servizio degli insegnanti, di qualsiasi ordine e grado. In nessuno dei percorsi formativi del nostro Paese, universitari e post-universitari, rivolti agli insegnanti - sia curricolari, sia specializzati per il sostegno - sono previsti corsi/insegnamenti specifici finalizzati allo sviluppo di conoscenze e abilità personali utili a promuovere, insieme alle competenze disciplinari, una maggiore efficacia dell'azione didattica e, dunque, un maggiore impatto sui processi di apprendimento degli studenti e sulla qualità delle relazioni interpersonali nel contesto classe. 
È necessario e quanto mai urgente promuovere le capacità sociali, emotive e comunicative degli insegnanti, includendole, sia nella loro formazione diretta (iniziale e in servizio), sia come costante attenzione riflessiva ed autoriflessiva durante l'esercizio della professione.

- Sul piano della formazione, conoscere l'impatto delle abilità personali sugli apprendimenti, sul clima sociale ed emotivo della classe, sulle relazioni con gli altri (studenti, colleghi, famiglie, etc.) e saper agire di conseguenza - come rileva la numerosa letteratura a supporto -, può davvero modificare ed orientare profondamente la qualità delle relazioni educative e il successo formativo degli studenti;

- Sul piano della riflessività, prevedere momenti di riflessione e auto-riflessione guidata (ad esempio con figure esperte) sulle proprie abilità personali, così come condividere con i colleghi esperienze, pensieri ed emozioni direttamente connessi all'insegnamento, nelle sue più complesse e articolate dinamiche (anche conflittuali), può aiutare sicuramente i docenti a comprendere la realtà da più punti di vista, chiarire equivoci, spiegare eventi complessi, risolvere o arginare situazioni problematiche in modo più efficace.

Nella formazione dell'insegnante, entrano così tanti aspetti, che non riguardano solamente una conoscenza approfondita della disciplina insegnata e della didattica, ma si riferiscono anche alla dimensione comunicativa, relazionale ed emotiva. È quanto mai importante - per le caratteristiche attuali della scuola - che tali dimensioni comincino a destare sempre più la loro attenzione nel campo delle politiche formative nazionali, con ricadute visibili sugli studenti, in particolare su coloro che a vario titolo si allontanano prematuramente dai percorsi scolastici, perché in questi non hanno trovato opportunità di sperimentare il successo formativo o proprio quell'accoglienza emotiva e relazionale di cui, a vari livelli, hanno bisogno.

\section{Contesti di apprendimento attivi per contrastare la di- spersione scolastica}

La dispersione scolastica è una problematica che interessa tutti i paesi della Comunità europea, infatti la Commissione Ue nel 2010 ha presentato una nuova strategia per ridurre il tasso di abbandono scolastico, almeno del $10 \%$, da raggiungere entro il 2020. Gli stati membri per mettere in atto la 
Strategia 2020 sono obbligati a presentare un Programma Nazionale individuando traguardi nazionali e obiettivi a medio termine. In Italia con il D.L. 15 aprile 2005 n. 76 è stato istituito un Sistema Nazionale delle Anagrafi degli studenti (ANS) attuato con D.M 74/2010, per cercare di monitorare e contrastare la dispersione scolastica. Dai dati pubblicati dal Miur a novembre 2017 sulla dispersione scolastica nell'a.s. 2015/16 e nel passaggio all'a.s. 2016/17 degli studenti italiani, si evince una situazione non certo rassicurante. Il proposito di questo contributo non è quello di commentare i dati emersi rispetto a numeri e percentuali tra nord, centro e sud Italia, o tra l'Italia e gli altri paesi della Comunità europea, quanto quello di capire e avviare una riflessione sulle diverse tipologie di abbandoni e le possibili iniziative da intraprendere per contrastare il problema.

Non è corretto parlare tout court di dispersione scolastica perché, oltre all'abbandono scolastico che si verifica in corso d'anno, il Focus pubblicato dal Miur individua le seguenti tipologie di studenti che escono dal sistema scolastico nazionale:

«1. alunni che frequentano la scuola secondaria di I grado e che interrompono la frequenza senza valida motivazione prima del termine dell' anno (abbandono in corso d'anno);

2. alunni che hanno frequentato l'intero anno scolastico (I e II anno di corso della scuola secondaria di I grado) e che non passano nell'anno successivo né al II o III anno in regola, né al I e II anno come ripetenti, e non passano alla scuola secondaria di II grado (abbandono tra un anno e il successivo);

3. alunni che hanno frequentato l'intero anno scolastico (III anno di corso della scuola secondaria di I grado) e che non passano nell'anno scolastico successivo alla scuola secondaria di II grado né frequentano nuovamente la scuola secondaria di I grado come ripetenti del III anno di corso (abbandono tra un anno e il successivo nel passaggio tra cicli scolastici);

4. alunni che frequentano la scuola secondaria di II grado e che interrompono la frequenza senza valida motivazione prima del termine dell' anno (abbandono in corso d'anno);

5. alunni che hanno frequentato l'intero anno scolastico (dal I al IV anno di corso della scuola secondaria di II grado), che non passano nell'anno successivo né al II, III, IV, V anno in regola, né al I, II, III e IV anno come ripetenti (abbandono tra un anno e il successivo)» (Miur, 2017, p. 11).

Viene spontaneo chiedersi: perché? Cosa accade, o non accade, dentro le aule scolastiche da spingere lo studente ad abbandonarle?

Negli anni le cause sono state attribuite a diversi fattori, partendo dal contesto socio-economico-culturale di appartenenza si è passati alla dimensione 
motivazionale del soggetto, per arrivare alla dimensione relazionale docentestudente. Oggi l'attenzione va focalizzata su un approccio multifattoriale: «un'esperienza in cui gli esiti (i risultati) non possono essere disgiunti dalle motivazioni, dai significati, dal sistema delle interazioni che influenzano i comportamenti. In altri termini al centro dell'attenzione vanno collocati sia il soggetto, inteso come attore capace di partecipare, di elaborare significati ed esperienze, sia i processi sociali che influenzano i percorsi formativi, coniugando così, gli aspetti individuali e relazionali con quelli strutturali» (Batini, 2016, p. 12).

Il panorama è ampio e complesso pertanto l'attenzione sarà rivolta, in modo particolare, all'aspetto metodologico didattico e al ruolo del docente e dello studente al fine di motivare, interessare, coinvolgere, rendere partecipe e attivo colui che deve essere il co-costruttore del proprio processo di apprendimento. Questa strada è dettata dalla convinzione che quello che accade dentro le aule scolastiche è così distante e lontano da ciò che accade fuori, da spingere i ragazzi verso le diverse tipologie di abbandono. Castoldi utilizza la metafora dell'insegnamento muro-ponte per evidenziare questa distanza, infatti il muro simboleggia un tipo di insegnamento dove «il sapere scolastico tende a seguire una sua logica fondativa ed evolutiva, legata alla progressione di contenuti e alla sistematicità dei curricoli, senza preoccuparsi troppo se incrocia l'esperienza di vita del soggetto, (...) la metafora del ponte evidenzia bene la logica sottesa a tale visione dell'insegnamento, tesa a strutturare continui processi di andata e ritorno tra esperienza e riflessione, sapere pratico e sapere teorico, fenomeno e rappresentazione» (Castoldi, 2011, pp. 50-52).

Contenuti astratti e inerti non spendibili nella vita reale non interessano più, è arrivato il momento nella scuola, di apportare cambiamenti non di facciata, ma il «cambiamento di tipo 2» (Watzlawick, Weakland e Fisck, 1974) che obbliga a ripensare i paradigmi del sistema e del processo educativo e formativo. Non possiamo affidarci solo a leggi e normative per rinnovare e cambiare, dobbiamo invece ripensare in profondità la scuola, mettendo in discussione teorie e metodologie; il ruolo degli attori del processo di insegnamento-apprendimento (docente-studente); il setting, e l'ambiente di apprendimento. L'attenzione andrebbe posta anche sulla qualità di ciò che si fa in aula e l'effetto che produce sugli studenti. La professionalità docente è fondamentale in questo, perché non basta padroneggiare la disciplina, quanto invece essere insegnanti ispirati, come li definisce Hattie, che «non usano i voti come punizione, non confondono la prestazione comportamentale con quella scolastica; non considerano l'obbedienza silenziosa più importante dell'apprendimento; non usano troppe schede preconfezionate; non hanno 
basse aspettative (...) non preferiscono la perfezione nello svolgimento dei compiti all'assunzione dei rischi, compreso quello di sbagliare» (Hattie, 2016, p. 82).

Queste argomentazioni obbligano a ripensare l'azione didattica sotto l'aspetto metodologico, relazionale-comunicativo e organizzativo. Le regole dello stile giornalistico anglosassone delle cinque $\mathrm{W}$, Who, Where, What, When, Why, che garantiscono un'informazione completa e adeguata ai lettori, in ambito educativo aiutano invece a progettare un'azione didattica rispondente ai bisogni degli studenti. È necessario sapere a chi (Who) è rivolta l'azione didattica, chi sono le persone coinvolte e che ruoli hanno; il dove (When) per sottolineare l'importanza dello spazio-contesto all'interno del quale l'azione didattica si svolge; che cosa (What) per pensare quali saperi selezionare affinché siano significativi per quel destinatario o gruppi di destinatari; quanto tempo (When) è necessario per quell'attività, ma soprattutto di quanto tempo necessita il soggetto che apprende; il perché (Why), quale il senso e il significato che l'insegnante e lo studente attribuiscono a quell'azione. È obbligo aggiungere il come (How) fondamentale in ambito didattico per definire attraverso quali metodologie, mezzi, strumenti e materiali realizzare il processo di insegnamento-apprendimento. Va creato un ambiente di apprendimento ottimale, un clima relazionale positivo e attento alla persona, invece le classi sono sempre più dominate da insegnanti che parlano, quando «ai fini dell'apprendimento, bisognerebbe parlare molto meno e ascoltare molto di più» (Hattie, 2016, p. 137). La lezione tradizionale si limita a dispensare conoscenze, mentre andrebbe facilitata nello studente l'attivazione di processi di riflessione sulle conoscenze già acquisite, per poterle mettere in campo. La conoscenza non può essere incapsulata dentro le discipline, ma deve generare da contesti reali e ritornare su di essi, «in una relazione ricorsiva tra esperienza e conoscenza, teoria e pratica» (Castoldi, 2011 , p. 50) e questo si può realizzare solo attraverso metodologie attive che mettano al centro del processo di apprendimento lo studente.

Necessita ripensare il processo di insegnamento-apprendimento, partendo dal pensiero di Lave e Wenger (1991), che ha influenzato in modo determinante lo sviluppo del concetto di apprendimento, dando così l'avvio a nuove prospettive, in particolare, quella dell'apprendimento situato, così definito, perché ancorato al contesto e alle attività che lo generano. Prospettive caratterizzate da un approccio metodologico-prassico, che partendo da problemi reali, tentano di trovare soluzioni, attraverso la sperimentazione e la ricerca, che possono essere senza dubbio una spinta innovativa per la scuola, «una modalità specifica con cui impostare i processi didattici, che 
vede nell'attività e nella capacità creativa del soggetto che apprende il motore necessario agli eventi che creano l'esperienza» (Cerri, 2012, p. 229).

Attraverso le metodologie attive è possibile fare attività di simulazione, che permettono di «riprodurre in un contesto protetto e controllato, esperienze simili a quelle del mondo reale per fornire agli studenti la possibilità di agire e apprendere dalle conseguenze delle proprie azioni» (Bonaiuti, 2014, p. 72). Si valorizza la capacità a pensare ed agire in modo autonomo all'interno di un ambiente significativo, in cui, la complessità delle parti che lo costituiscono sono inseparabili (Morin, 2000) e nel quale emerge la progettazione dell'ambiente formativo, in un'ottica ecologico-sistemica, fondata su relazioni tra persone e oggetti, in modo ricorsivo. In questo contesto l'apertura al nuovo, la capacità decisionale, la possibilità di percorrere strade diverse per la risoluzione del problema, la giusta distanza tra il noto e l'ignoto (Tessaro, 2002), sono gli elementi che generano la conoscenza in azione.

L'aula diventa il luogo della sperimentazione e del confronto; contesto di apprendimento privilegiato; comunità di ricerca in cui le parti che entrano in relazione si arricchiscono, si incentivano le dinamiche relazionali, dalle quali nasce la cooperazione e la condivisione, per dare significato all'azione. Le metodologie attive generano l'interazione tra pensiero e azione e la possibilità di elaborare, rielaborare, costruire, decostruire, aiuta a trovare soluzioni innovative. Ad esempio la didattica laboratoriale non si affida a percorsi precostituiti, ma attraverso la ricerca-azione, metodo aperto all'incertezza e al possibile, segue le logiche reticolari, facendo ricorso ad una metodologia plurale; individuando così, ogni volta, quella che risponde alle esigenze di un contesto e di un percorso in quanto «la pratica del plurale è in stretta equazione con lo stile sperimentale» (Frabboni, 2005, p. 47).

In questo modo il processo di insegnamento apprendimento «si fa sempre più dialogo nel gruppo e sempre meno racconto dell'insegnante» (Gherardi, 2013, p. 41). Il laboratorio diventa uno spazio mentale attrezzato, con metodi e strumenti specifici, caratterizzato dalla trasversalità e dall'interdisciplinarità. Il laboratorio con la sua morfologia polifunzionale, accoglie, motiva, soddisfa interessi, facilita la comunicazione, la socializzazione, l'autonomia, la fantasia, sviluppa il pensiero divergente, facilita l'autoapprendimento, invitando alla scoperta. In questo quadro il laboratorio diventa il luogo nel quale si supera la logica del disciplinarismo e si recupera l'unitarietà del sapere, permettendo di unire mano e mente, di unire il sapere con il fare, dando senso al fare attraverso la riorganizzazione delle conoscenze (De Santis, 2016). È necessario che si sviluppi quella epistemologia della pratica che 
abitua alla riflessione, condizione che permette di organizzare il proprio apprendimento e quindi di imparare ad imparare, che insieme all'autoregolazione, «induce ad assumere un ruolo meno passivo» (Calvani, 2011b, p. 87) e quindi costruire la propria conoscenza. Quindi un percorso formativo basato sull'esperienza, permette di acquisire consapevolezza sul pensiero e sull'azione, infatti «essere impegnati in un laboratorio dove si è chiamati a esplicitare la propria epistemologia sviluppa la disposizione a stare con uno sguardo vigile nella realtà» (Mortari, 2013, p. 51). Rinnovare la didattica potrebbe essere un primo passo per non allontanare ma "agganciare" (Wiggins e McTighe, 2004) e motivare lo studente al fine di sviluppare la comprensione profonda, che si concentra «sul riconoscere e appropriarsi dell'orizzonte di senso di un dato sapere» (Castoldi, 2017, p. 103), per essere speso nella vita reale. La qualità dell'insegnamento e dell'apprendimento dipende quindi da molti fattori, ma soprattutto da scelte metodologiche e didattiche. Gli insegnanti andrebbero formati all'utilizzo delle metodologie attive, andrebbe ripensata l'intera formazione docente, poiché molti studi hanno evidenziato l'impatto deludente di percorsi fin ora istituiti (Korthagen, 2004). Dall'altro lato, però non ci sono studi che dimostrano che migliorando la formazione universitaria degli insegnanti automaticamente migliori la qualità dell'insegnamento. Quella che va cambiata è la cultura della scuola e la forma mentis degli insegnanti, che devono essere attenti non solo ad essere "efficaci" ma all'entità e alla natura degli effetti che le loro azioni hanno sugli studenti. Devono offrire: opportunità diverse affinché tutti possano apprendere; aiutare a tenere vivo l'interesse per l'apprendimento; coinvolgere in nuove esperienze di apprendimento; creare possibilità di crescita e non porsi come ostacoli; essere consapevoli che per far apprendere non bisogna scomporre il sapere, ma dare "compiti sfidanti". Affinché si sviluppi una nuova "forma mentis" degli insegnanti è necessario promuovere le seguenti convinzioni:

- «tutti gli studenti possono essere sfidati;

- è sempre questione di strategie, mai di stili;

- è importante avere alte aspettative verso tutti gli studenti, tenendo conto del loro punto di partenza;

- è importante incoraggiare i comportamenti di richiesta di aiuto;

- è importante insegnare a tutti gli studenti una varietà di strategie di apprendimento;

- è importante formare studenti capaci di valutare;

- per migliorare l'apprendimento è importante sviluppare l'interazione tra pari; 
- senso critico, errore e feedback sono opportunità potenti per migliorare l'apprendimento;

- sviluppare l'autoregolazione degli studenti e aiutarli a diventare "insegnanti di se stessi”" è importantissimo per migliorare l'apprendimento;

- non dare la colpa ai ragazzi;

- gli svantaggi che derivano dallo status socioeconomico della famiglia posso essere superati;

- non c'è spazio per il pensiero disfunzionale, quindi non si etichettano gli studenti e non si hanno basse aspettative nei loro confronti» (Hattie, 2016, p. 261).

Convinzioni che confermano ancora il valore che hanno le metodologie attive nel processo di insegnamento-apprendimento, in quanto richiamano molti degli aspetti che le caratterizzano: il ruolo attivo, consapevole e responsabile dello studente; il valore dell'interazione sociale; l'importanza di procedere per tentativi ed errori e la costruzione sociale della conoscenza nel processo di insegnamento-apprendimento.

\section{Riferimenti bibliografici}

Batini F. (2016), Un panorama desolante, in Batini F. e Bartolucci M., a cura di, Dispersione scolastica. Ascoltare i protagonisti per comprenderla e prevenirla, FrancoAngeli, Milano.

Bonaiuti G. (2014), Le strategie didattiche, Carocci, Roma.

Calvani A. (2011a), “«Decision Making» nell'istruzione. «Evidence Based Education» e conoscenze sfidanti”, Journal of Educational, Cultural and Psychological Studies, 3: 77-99.

Calvani A. (2011b), Principi dell'istruzione e strategie per insegnare. Criteri per una didattica efficace, Carocci, Roma.

Calvani A. (2012), Per un'istruzione evidence based. Analisi teorico-metodologica internazionale sulle didattiche efficaci e inclusive, Erickson, Trento.

Calvani A. (2014), Come fare una lezione efficace, Carocci, Roma.

Castoldi M. (2011), Progettare per competenze. Percorsi e strumenti, Carocci, Roma.

Castoldi M. (2017), Costruire unità di apprendimento. Guida alla progettazione a ritroso, Carocci, Roma.

Catalano M.G., Perrucchini P., and Vecchio G.M. (2014), “The quality of teachers'educational practices: internal validity and applications of a new self-evaluation questionnaire", Procedia-Social and Behavioral Sciences, 141: 459-464, testo disponibile al sito:

http://www.sciencedirect.com/science/article/pii/S1877042814035046 - Consultato in data 31-01-2018.

Cerri R., a cura di (2012), L'evento didattico. Dinamiche e processi, Carocci, Roma.

Concina E. (2016), "L'insegnante efficace: definizione e caratteristiche nella ricerca educativa”, Form@re, 2, 16: 20-31, testo disponibile al sito: http://dx.doi.org/10.13128/formare-18200 - Consultato in data 02/02/2018. 
Cottini L. e Morganti A. (2015), Evidence-based education e pedagogia speciale: principi e modelli per l'inclusione, Carocci, Roma.

Cottini L. and Morganti A. (2016), "Does the school inclusion really work? ” Education Sciences \& Society, 7: 13-32, ISSN: 2038-9442, testo disponibile al sito: http://dx.doi.org/10.3280/ess1-2016oa3240 - Consultato in data 25/01/2018.

De Santis M. (2016), Il laboratorio. Per una didattica ludica della formazione, Aracne, Roma. Frabboni F. (2005), Il laboratorio per imparare ad imparare, Tecnodid, Napoli.

Gherardi V. (2013), Metodologie e didattiche attive. Prospettive teoriche e prospettive operative, Aracne, Roma.

Hattie J. (2012), Visible learning for teachers. Maximizing impact on learning, Routledge, London (trad. it: Apprendimento visibile, insegnamento efficace, Erickson, Trento, 2016).

Jennings P. A. and Greenberg M. T. (2009), "The prosocial classroom: Teacher social and emotional competence in relation to student and classroom outcomes" Review of educational research, 79, 1: 491-525.

Korthagen F.A.J. (2004), "In search of the essence of a good teacher: towards a more holistic approach in teacher education", Teaching and Teacher Education, 20, 1: 77-97.

Lave J. and Wenger E. (1991), Situated learning: Legitimate peripheral participation, Cambridge University Press, Cambridge.

Mitchell D. (2016), Diversities in education: Effective ways to reach all learners, Routledge, London, NY.

Miur (2017), La dispersione scolastica nell'a.s 2015/16 e nel passaggio all' 2016/17, Ufficio Statistica e Studi, testo disponibile al dito: http://www.miur.gov.it/documents/20182/0/Focus+La+dispersione+scolastica/9bc1c11b-1c40-4029-87ba40b8ba2bc2bf?version=1.1 - Consultato in data 30/01/2018.

Morganti A. (2012), "Evidence Based Education e didattica speciale: un inquadramento", American Journal on Intellectual and Developmental Disabilities, 10: 315-327.

Morganti A. e Bocci F., a cura di (2017), Guida: Didattica Inclusiva per la Scuola Primaria. Educazione socio-emotiva e Apprendimento cooperativo per costruire competenze inclusive attraverso $i$ "compiti di realtà, Giunti EDU, Firenze.

Morganti A. e Signorelli A. (2017), "La didattica inclusiva: la sfida di un'educazione "senza barriere", Psicologia e Scuola, 50: 1-8.

Morin E. (2000), La testa ben fatta, Cortina, Milano.

Mortari L. (2013), Cultura della ricerca e pedagogia. Prospettive epistemiche, Carocci, Roma.

Rubio M.C. (2009), "Effective teachers -Professional and personal skills", ENSAYOS, Revista de la Facultad de Educación de Albacete, 24, testo disponibile al sito: http://www.uclm.es/ab/educacion/ensayos - Consultato in data 30-01-2018.

Tessaro F. (2002), Metodologia e didattica dell'insegnamento secondario, Armando, Roma. Vivanet G. (2013), "Evidence Based Education: un quadro storico", Form@re, 2, 13: 41-51. Vivanet G. (2014), Cos'è l'Evidence Based Education, Carocci, Roma.

Watzlawick P., Weakland J. H. and Fisck R. (1974), Change, Astrolabio, Roma.

Wiggins G. e McTighe J. (2004), Fare progettazione. La teoria di un percorso didattico per la comprensione significativa. LAS, Roma (ed. or. 1998). 


\title{
Dispersione scolastica: lo studio, un dovere e un diritto
}

\author{
di Giovanna Farinelli
}

From couch to coach

\section{Premessa}

Nella sua prolusione alla cerimonia di inaugurazione dell'anno accademico 2017/2018 dell'Accademia Anatomico-Chirurgica di Perugia, lo scorso 15 febbraio, Marco Catani, docente di neuroanatomia funzionale presso il King's College di Londra, ha sottolineato come dalle più recenti ricerche nel suo NatBrainLab - Laboratorio di Neuroscienze continui a emergere la necessità, per lo sviluppo umano, di parlare con i bambini e di favorire un atteggiamento pro sociale, cioè di socialità e condivisione.

Lo studio delle connessioni cerebrali e dei fascicoli neuronali sembra dimostrare come l'udito sia l'hub più importante per il funzionamento del cervello attraverso il linguaggio come strumento principe non soltanto della comunicazione ma della implementazione e creazione della cultura fin dalle origini. Ci si domanda infatti perché lo scimpanzé, pur essendo geneticamente quasi simile all'uomo, non parli ancora. Le nuove tecnologie digitali, eccezionalmente visuali, non implementano la cultura o l'umanità ma isolano, non sfruttando appieno le capacità del cervello umano nelle sue connessioni se parola e udito non funzionano compiutamente: il linguaggio è dunque una possibilità ma soprattutto una necessità, un dovere. Sembrerebbe confermata dunque l'affermazione di Platone nel Fedro (274c), per cui la scrittura stessa è veleno per la memoria, a sostegno di una oralità che ha permesso e permette ancora la sopravvivenza e il progresso della specie umana. Rita Levi Montalcini afferma «la disparità evolutiva dei sistemi cerebrali preposti alle facoltà cognitive ed emotive» e spiega che «i figli dell'uomo differiscono da quelli di altri mammiferi nella lentezza del loro sviluppo somatico e intellettuale, che li rende dipendenti dai genitori o da chi ne fa le veci»; per questo il periodo dell'imprinting nell'uomo si estende per tutta la durata della vita e così il linguaggio «rappresenta il maggior privilegio che 
sia stato dato all'uomo e che gli ha aperto gli infiniti orizzonti del pensiero» (Levi Montalcini, 1989, pp. 222-223).

La maturità psicosociale sembra essere inversamente proporzionale, col progresso dell'umanità, alla maturità biologica; si evidenzia infatti un $m i$ smatch of biological and psychological/social transitions, come affermano George C. Patton e Russell Viner (2007).

\section{Precisazione linguistica}

Il fenomeno della dispersione scolastica, di abbandono della scuola, tra bocciatura e ripetenza (rejection), di insuccesso e fallimento formativo (in inglese semplicemente consequences); ovvero di school desertion, leaving or abandonment, si sintetizza in un'espressione/locuzione che è ormai quasi uno slogan (cfr. Reboul, 1977): drop out, i cosiddetti "nonstudianti" (Mastrocola, 2011), siano questi NEET (Not Employed in Education or Training), ESL (Early School Leavers) o ELET (Early Leavers from Education and Training), in Italia ben superiori al 10\%, obiettivo stabilito dall'UE per il 2020, con la magra consolazione che l'Umbria risulta però l'ultima regione italiana per tasso di abbandono dunque la più virtuosa (Miur, 2017; 2018).

Altri concetti che in lingua italiana definiscono tale fenomeno sono per esempio elusione ed evasione dell'obbligo scolastico/formativo: si noti la scelta e ricorrenza del termine "obbligo" (relativo, specifico), eticamente e giuridicamente diverso dal termine "dovere" (assoluto, generale). Altra espressione è quella di fallimento formativo, molto spesso legato a fenomeni di assenteismo, corruzione, marginalità, esclusione e disagio sociale, criminalità, verosimilmente in presenza di genitori ed educatori compiacenti e in assenza di controllo sociale e del territorio (per un approfondimento delle diverse accezioni del fenomeno si veda Benvenuto, 2011). Viene meno la fiducia nelle istituzioni (Trust in Governance, UE Horizon 2020), il senso di appartenenza allo Stato e l'identità umana ipse, che significa cive e non soltanto omo (Dante, Paradiso, VIII, 116).

Un curioso apparentamento/parallelismo si mostra infatti, almeno in Italia, con i fenomeni/termini di elusione ed evasione del fisco, nonostante si reputi eccessiva la pressione fiscale nel nostro Paese. Tutto ciò senza tener conto che è proprio il gettito fiscale (fiscal drag), obbligo materiale, che consente l'erogazione dei servizi essenziali tra cui l'esercizio del diritto all'istruzione e alla salute che consentono di attuare il dovere inderogabile di solidarietà politica, economica e sociale (articolo 2 della Costituzione Italiana). Ciò 
significa riconoscere allo studio un primato, che diventa dovere civico e morale per i capaci ma soprattutto/in particolare per i meritevoli: i capaci e meritevoli hanno infatti il dovere oltre che «il diritto di raggiungere $i$ gradi più alti degli studi» (articolo 34 della Costituzione Italiana).

Studio e lavoro si possono riassumere in una parola: occupazione (work, job) come dovere e come diritto, oltreché obbligo/necessità e possibilità/libertà. Simone Weil, nel testo La prima radice. Preludio ad una dichiarazione dei doveri verso l'essere umano, pubblicato postumo nel 1949, afferma infatti che se la disoccupazione è «uno sradicamento alla seconda potenza», il secondo fattore è «l'istruzione quale è concepita al giorno d'oggi» (Weil, 1990, p. 50).

Secondo quanto afferma Federico Batini (2013), «i drop-out sono ragazzi e ragazze che, nella quasi totalità dei casi, hanno un rapporto difficile e complesso con il concetto stesso di stare in aula»: normalmente sono definiti in negativo. Il problema potrebbe infatti dipendere dalla certificazione stessa, onere e onore che spetta alla scuola, e dai cosiddetti programmi, «stella polare dei processi di insegnamento» rispetto alla «centralità dell'apprendimento e dei soggetti» (Batini, 2013; cfr. Batini, 2014; MIUR, 2018).

In sintesi, il termine dropout in Merriam-Webster.com (4 marzo 2018) indica due significati fondamentali: 1a) one who drops out of school; 1b) one who drops out of conventional society; 1c) one who abandons an attempt, activity, or chosen path; a corporate dropout; 2a) momentary defect on a magnetic tape or disk caused by a temporary loss of signal (prima attestazione del termine: 1930).

Lo Zingarelli 2002 e l'ultima edizione 2018 riportano lo stesso significato di dropout: «Chi abbandona la scuola prima di ottenere il diploma» o, estensivamente, raro, «chi vive in uno stato di emarginazione» (prima attestazione: 1980-1976).

Nella pubblicazione MIUR 2017 (pp. 21, 25) si afferma che, «considerando il dettaglio della cittadinanza degli alunni, è evidente come il fenomeno della dispersione scolastica colpisca maggiormente i cittadini stranieri rispetto a quelli italiani... Se si analizza il fenomeno dal punto di vista della regolarità nel percorso di studi, spicca la percentuale nettamente più elevata per coloro che sono in ritardo, a dimostrazione del fatto che la ripetenza può essere considerato un fattore che precede, e in certi casi preannuncia, l'abbandono». Nell'ambito della secondaria, in particolare, «il ritardo si accumula soprattutto negli istituti professionali e tecnici.» (MIUR, 2018, p. 25). 


\section{Dovere/diritto allo studio e inclusione sociale}

In Italia l'istruzione è obbligatoria per almeno 10 anni e riguarda la fascia di età compresa tra i 6 e i 16 anni. Tale adempimento (obbligo scolastico) è finalizzato al conseguimento di un titolo di studio di scuola secondaria superiore o di una qualifica professionale di durata almeno triennale entro i 18 anni. L'istruzione obbligatoria è altresì gratuita; tale obbligo può essere assolto nelle scuole statali e paritarie; nelle strutture accreditate dalle Regioni per la formazione professionale; attraverso l'istruzione parentale (home schooling). L'obbligo formativo è il diritto/dovere dei giovani che hanno assolto all'obbligo scolastico di frequentare attività formative fino all'età di 18 anni.

La Legge Casati (Legge 3725), dal nome del Ministro per la Pubblica Istruzione del Regno di Sardegna, viene emanata il 13 novembre 1859 e redatta da Vittorio Emanuele II; dopo la proclamazione del Regno d'Italia (1861) viene estesa gradualmente all'intero Paese. Si precisa che l'obbligo scolastico dei primi due anni (scuola elementare inferiore) può essere assolto - con "comodo" dei genitori - anche attraverso la cosiddetta "scuola paterna". La legge Coppino (Legge 3961), approvata il 15 luglio 1877, istituisce/sancisce l'obbligatorietà dell'istruzione elementare inferiore fino al nono anno di età (Bertonelli e Rodano, 2003). Il Rapporto MIUR 2018 (p. 37) sottolinea in proposito come l'Italia unitaria, proprio dalla Legge Casati, abbia avuto «il merito storico di promuovere ininterrottamente l'ascensore sociale grazie all'istruzione, con la diffusione delle scuole pubbliche».

Un aggiornamento significativo si ha con la Legge 31 dicembre 1962, n. 1859 , sull'istituzione e ordinamento della scuola media statale in cui l'obbligo scolastico prevede un percorso di 8 anni. La Legge 28 marzo 2003, n. 53 (Legge Moratti), che aggiorna la definizione di scuola materna ed elementare in scuola dell'infanzia e primaria (articolo 2, comma 1d), sostituisce l'espressione "obbligo scolastico" con quella di «diritto-dovere all'istruzione e alla formazione» (articolo 1, comma 1i), assicurando il diritto all'istruzione e alla formazione per almeno 12 anni e comunque sino al conseguimento del diciottesimo anno di età.

L'articolo 34 della Costituzione Italiana (1 gennaio 1948) afferma che «la scuola è aperta a tutti. L'istruzione inferiore, impartita per almeno otto anni, è obbligatoria e gratuita. I capaci e meritevoli, anche se privi di mezzi, hanno diritto di raggiungere i gradi più alti degli studi. La Repubblica rende effettivo questo diritto con borse di studio, assegni alle famiglie ed altre provvidenze, che devono essere attribuite per concorso»». 
Sempre nella prima parte della Costituzione Italiana, Titolo II, Rapporti etico-sociali; nell'articolo $30 \mathrm{si}$ afferma il dovere e il diritto dei genitori «di mantenere, istruire ed educare i figli... Nei casi di incapacità dei genitori, la legge provvede a che siano assolti i loro compiti». La legge 219 del 2012, art. 2, comma 1, nell'affermare la «unificazione delle disposizioni che disciplinano i diritti e i doveri dei genitori nei confronti dei figli nati nel matrimonio e dei figli nati fuori del matrimonio», delinea la «nozione di responsabilità genitoriale quale aspetto dell'esercizio della potestà genitoriale». Questa affermazione è fondamentale, poiché sostituisce il termine potestà con il concetto etico e pedagogico di responsabilità.

Nella Convenzione ONU sui diritti dell'Infanzia (1989) al comma 1 dell'articolo 18 si afferma infatti che «gli Stati parti faranno del loro meglio per garantire il riconoscimento del principio secondo il quale entrambi i genitori hanno una responsabilità comune per quanto riguarda l'educazione del fanciullo e il provvedere al suo sviluppo. La responsabilità di allevare il fanciullo e di provvedere al suo sviluppo incombe innanzitutto ai genitori oppure, se del caso, ai suoi tutori legali i quali devono essere guidati principalmente dall'interesse preminente del fanciullo».

Con la Legge 28 marzo 2001, n. 149 si sottolinea in particolare il diritto del minore alla propria famiglia (articolo 1). In seguito ai massicci fenomeni migratori degli ultimi anni, la recente Legge 7 aprile 2017, n. 47 (Disposizioni in materia di misure di protezione dei minori stranieri non accompagnati) individua e tutela una tipologia di bambini, i cosiddetti MSNA (minori stranieri non accompagnati), che spesso sfuggono fisicamente alla rete dei servizi che dovrebbe occuparsi di loro e non soltanto alla cosiddetta Anagrafe dello Studente (MIUR, 2018).

A conferma della continuità e della necessità di un contesto socio-familiare ai fini dell'attuazione del dovere/diritto allo studio, soprattutto per i minori, la stessa Legge 5 giugno 1990, n. 148 (Riforma dell'ordinamento della scuola elementare), all'articolo 1, comma 1, afferma che «la scuola elementare, nell'ambito dell'istruzione obbligatoria, concorre alla formazione dell'uomo e del cittadino secondo i principi sanciti dalla Costituzione e nel rispetto e nella valorizzazione delle diversità individuali, sociali e culturali. Essa si propone lo sviluppo della personalità del fanciullo, promuovendone la prima alfabetizzazione culturale» (comma 1). «La scuola elementare, anche mediante forme di raccordo pedagogico curricolare ed organizzativo con la scuola materna e con la scuola media, contribuisce a realizzare la continuità del processo educativo» (comma 2 ).

È infatti interessante sottolineare come la stessa legge che introduce il cosiddetto "modulo organizzativo", all'articolo 5, comma 5, affermi che «nei 
primi due anni della scuola elementare, per favorire l'impostazione unitaria e pre-disciplinare dei programmi, la specifica articolazione del modulo organizzativo, di cui all'articolo 4, è di norma, tale da consentire una maggiore presenza temporale di un singolo insegnante in ognuna delle classi». Le più recenti leggi in materia di scuola primaria enfatizzano questa "prevalenza" della figura del docente, che peraltro nel percorso formativo in Italia non ha mai previsto - pur nella sostanziale modifica della durata dei percorsi stessi da Casati a oggi - la formazione di un insegnante disciplinare specializzato nell'ambito della scuola dell'infanzia e della primaria (Cfr. Bucci, 2000).

Vanno inoltre ricordati gli articoli 26 della Dichiarazione Universale dei diritti Umani (10 dicembre 1948) in cui al comma 1 si afferma che «ogni individuo ha diritto all' istruzione. L'istruzione deve essere gratuita almeno per quanto riguarda le classi elementari e fondamentali. L'istruzione elementare deve essere obbligatoria. L'istruzione tecnica e professionale deve essere messa alla portata di tutti e l'istruzione superiore deve essere egualmente accessibile a tutti sulla base del merito»; il comma 2 afferma che «l'istruzione deve essere indirizzata al pieno sviluppo della personalità umana ed al rafforzamento del rispetto dei diritti umani e delle libertà fondamentali. Essa deve promuovere la comprensione, la tolleranza, l'amicizia fra tutte le Nazioni, i gruppi razziali e religiosi, e deve favorire l'opera delle Nazioni Unite per il mantenimento della pace». Al comma 3: «I genitori hanno diritto di priorità nella scelta del genere di istruzione da impartire ai loro figli»e all'art. 16 comma 3: «La famiglia è il nucleo naturale e fondamentale della società e ha diritto ad essere protetta dalla società e dallo Stato».

Dunque il dovere/diritto allo studio è dunque uno strumento essenziale per la prevenzione della dispersione: la legge prevede infatti, a sostegno, interventi di «orientamento contro la dispersione scolastica» e per assicurare la realizzazione del diritto-dovere di istruzione e formazione (Legge $53 / 2003$, art 1 comma 3i). Si sottolinea di nuovo il ruolo fondamentale del contesto familiare e sociale (cfr. art. 16, Legge 8 novembre 2000, n. 328, "Legge quadro per la realizzazione del sistema integrato di interventi e servizi sociali").

Come non richiamare nel contesto del Trattato sull'Unione europea e del Trattato sul funzionamento dell'Unione europea (1998-2017), la Carta dei Diritti Fondamentali dell'Unione Europea, che nel Titolo II Libertà, all'articolo 14, Diritto all'istruzione, afferma il diritto di ogni persona «all'istruzione e all'accesso alla formazione professionale e continua».

La scuola dunque non costituisce più un obbligo ma rappresenta un dovere oltre che diritto di autorealizzazione attraverso la capacità di padroneggiare i principali codici: linguistico, matematico/numerico e giuridico. In 
questo contesto si colloca anche lo storico provvedimento di Aldo Moro che introduce nel 1958 (DPR 13 giugno 1958, n. 585) l'educazione civica a scuola così come la Legge del 30 ottobre 2008, n. 169 sull'insegnamento di "Cittadinanza e Costituzione".

Elusione, evasione, corruzione, sfiducia delle istituzioni pubbliche sono facce della stessa medaglia: conoscere e rispettare la legge, come ha ricordato di recente Nicola Gratteri, procuratore capo di Catanzaro (Radio anch'io, Radio 1, mercoledì 21 febbraio 2018; cfr. Gratteri, Nicaso, 2010).

Sarebbe infatti auspicabile, secondo il Rapporto MIUR 2018 (pp. 20, 29), l'istituzione e la manutenzione di «comunità educanti solide», che si mostrino «capaci di contrastare il fenomeno non solo del fallimento formativo ma anche del rischio di caduta nelle maglie della criminalità organizzata e non»; che le «singole istituzioni scolastiche si sappiano misurare con la prospettiva del potenziamento educativo fondato sulla costruzione di larghe comunità educanti in ogni singolo territorio, grazie alla partecipazione, su base paritaria eppure ben articolata per funzioni e responsabilità, a reti e veri e propri stabili partenariati educativi più ampi e che contengano centri sportivi, parrocchie, terzo settore, volontariato, saperi diffusi, etc.» anche attraverso una «cura meticolosa degli alfabeti di cittadinanza».

I nuovi analfabetismi riguardano infatti, più che il cosiddetto digitale, etica e valori, le emozioni e la capacità di lettura/comprensione della realtà, la capacita di comunicare-ascoltare e parlare - soprattutto della scuola - linguaggi condivisi, trasparenti e univoci, civili e politici. Le nuove tecnologie, troppo legate al tatto e alla vista, paradossalmente allontanano dai significati veri e non promuovono quell'avanzamento che tutta la nostra tradizione orale dalla Bibbia da Omero da Beowulf fino ai cantastorie, dall'epica alle ballate alle filastrocche alle canzoni all'opera ha promosso per almeno tre millenni (Ong, 1986).

Si conferma dunque il ruolo della scuola pubblica, come istituzione trasparente e democratica, che significa non solo «aperta a tutti» (articolo 34 della Costituzione Italiana), ma anche «volta a un fine collettivo, a un interesse pubblico», come afferma Ernesto Galli della Loggia (2017). Secondo lo stesso autore, «poche cose sono state altrettanto decisive nel formare... la nostra identità di italiani... una lezione di povertà, austera, di serietà e di disciplina»: questa scuola ha rappresentato «una sorta di riassunto (simbolico ma insieme reale) di ciò che voleva dire la cosa pubblica e per estensione lo Stato»; egli sottolinea in sintesi «quanto sia importante che ci siano istituzioni degne che funzionino, quanto conti l'esistenza di uno spirito pubblico orientato alla legalità, cittadini animati da un qualche senso civico» (Galli della Loggia, 2010, pp. 173-175). 
In tal senso si colloca anche il recente Horizon 2020 Work Programme della Commissione Europea per il triennio 2018-2020, Europe in a changing world - Inclusive, innovative and, in reflective societies, in particolare nella Call Governance for the Future: Trust in Governance (GOVERNANCE-0119): «Trust is a fundamental condition for a fair and cooperative society. It also plays an important part in contributing to social capital. While a degree of distrust may be required for a well-functioning democracy, waning trust in governments and other institutions and in the EU can impact European governance in multiple ways. The challenge is to restore and improve trust as a basis for sustainable and legitimate governance».

Nella lotta alla dispersione scolastica, al fallimento formativo, la fiducia nelle istituzioni è dunque essenziale. La famiglia, oltre alla scuola, deve garantire e rappresentare una esperienza/trasparenza autenticamente democratica e non di clan o lobby, cioè corporativa, tantomeno oligarchica. Federico Fubini, nella pubblicazione La maestra e la camorrista (2018, pp. 73-75) sottolinea come «non basta la qualità della famiglia, se poi cresci in un ambiente sbagliato... bambini che a tre anni presentano capacità completamente diverse, evidenti nel numero di parole che usano, in base al "bagno somatosensoriale"». Citando l'economista premio Nobel James Heckman (2013), Fubini (2018, pp. 17-18) riporta che «negli Stati Uniti un figlio di professionisti laureati a tre anni ascolta in media 2.153 parole l'ora e ne usa già 1.400; il figlio di operai ne ascolta 1.251 e ne usa 750 ; quello di disoccupati non riesce a controllarne più di $500 \ldots$ Le capacità umane si moltiplicano come cellule, per questo è essenziale partire con un patrimonio biologico ricco». Più avanti, sostiene che «deciderà molto di noi italiani questo rapporto fra eredità, riproduzione, istruzione, mobilità sociale e grado di fiducia negli altri» (Fubini, 2018, p. 19).

Le condizioni socio-economiche della famiglia giocano un ruolo importante nel determinare l'abbandono scolastico. I ragazzi provenienti da ambienti economicamente svantaggiati sono, infatti, più a rischio di abbandono scolastico, anche per le pressioni - esplicite o implicite - che ricevono per diventare il prima possibile indipendenti economicamente (Bradley e Renzulli, 2011).

Il cosiddetto amoral familism descritto da Edward C. Banfield (1958) o "familismo amorale" di cui parla Raffaele Cantone (2017, p. 80), prodromo/base della corruzione, è da contrastare agendo su tre piani/pilastri: repressione, prevenzione e educazione. La corruzione rappresenta infatti «il portato di una scarsa consapevolezza del valore collettivo della res publica e dei suoi beni», è una «malattia sociale, da combattere con l'affermazione di 
un nuovo modello di cultura, in particolare di un'etica delle regole, del merito e della concorrenza», grazie anche a una scuola «più aperta ai valori della legalità e dell'etica... una rivoluzione culturale tra $i$ banchi di una scuola aperta all'esterno a una cultura meno autoreferenziale» (Cantone, 2017, pp. 201-206): prima e dopo la scuola, famiglia e "agenzie culturali". Meno burocrazia, dunque, meno corruzione, secondo la ben nota equazione di Cantone.

Secondo Fubini, (2018, p. 9), che richiama la figura forse anacronistica e obsoleta del precettore (coach, in termini più attuali), l'immobilismo sociale è la «tragedia dei beni comuni», secondo la nota espressione dell'economista William Forster Lloyd. Collega fiducia, successo e autostima, quando afferma che «la demotivazione a scuola contribuisce a fare dell'Italia una società mediamente poco istruita» mentre «l'eccesso di autostima deve pur avere nella società una funzione simile all'amore in biologia, quando ti acceca per spingerti ad assicurare la continuazione della specie» (Fubini, 2018, pp. 55, 32). La nostra società rappresenta «il dramma delle corporazioni di ogni tipo che rifiutano di cedere terreno», «il dramma dei diseredati, che non pensano valga la pena di investire su se stessi attraverso gli studi»: è questa secondo Fubini, «una tossina che paralizza la società italiana» (pp. 32, 7475, 109, 124).

Autostima, autocontrollo e forza di volontà costituiscono forse «il nucleo da cui tutto si irradia»: la povertà educativa biologica e sociale porta a confondere il valore con il prezzo (Fubini, p. 109). L'Italia è una «società dinastica» caratterizzata dalla «diffidenza verso le istituzioni», che disincentiva creatività e accumulazione di conoscenza il cui carattere patrimoniale potrebbe rappresentare il problema oppure il sintomo (Fubini, pp. 124-125). Fubini sottolinea infatti come in Italia, se il valore dei patrimoni privati esplode, quello del patrimonio pubblico implode in modo uguale e contrario e prende forma l'enorme debito dello Stato (p. 109): «questo sistema di welfare distorto, che favorisce la crescita dei patrimoni delle persone più anziane, non può che aver depresso la natalità»; il calo demografico è dunque in relazione con la povertà educativa (p. 123).

Nello stesso Rapporto MIUR 2018 (pp. 30-31), che definisce il complesso paesaggio della scena educativa, entro spazi qualitativamente ridotti della socialità in cui è maggiore la solitudine dei bambini e ragazzi che, «fragili e spavaldi», crescono in «famiglie adolescenti», e minore è la capacità di autonomia, si sottolinea un'aspettativa di vita molto più lunga, genitori molto meno giovani di un tempo e tra i più vecchi del pianeta, giovani che vivono con intorno pochi coetanei e tanti adulti e vecchi: «abbiamo spesso un solo figlio [il bambino unico]... Quando le famiglie avevano due, tre o più figli, i 
bambini erano, per necessità della vita reale quotidiana, abituati a gestire gradi di frustrazione. Non erano il centro dell'universo degli adulti. Ora arrivano a scuola da una situazione di assoluta centralità e si trovano, spesso per la prima volta, uno tra tanti, uguale agli altri, a dividere sì un'avventura sociale comune, che è un piacere impagabile, ma anche a vivere le frustrazioni e le limitazioni del proprio io alle quali non sono abituati come una volta».

Tra le possibili soluzioni, secondo Fubini una scuola più efficace; l'obbligo scolastico a 18 anni - la propensione ad anticipare le scelte formative aumenta, infatti, il rischio di dispersione (Rumberger e Lamb, 2003); all'insegnamento solo i laureati migliori; cancellare il valore legale della laurea; borse di studio e sussidi; spendere più nell'educazione che nei patrimoni personali contro una mentalità di rendita o di tipo corporativo (Fubini, pp. 123128). È inoltre auspicabile la piena applicazione del dettato normativo sulla chiamata diretta dei docenti (Cfr. legge del 13 luglio 2015, n. 107) anche in vista di una liberalizzazione delle professioni.

\section{Rapporto MIUR 2018 in sintesi: una lettura della scuola a margine della XVII legislatura}

Il Rapporto Una politica nazionale di contrasto del fallimento formativo e della povertà educativa. Cabina di regia per la lotta alla dispersione scolastica e alla povertà educativa (10 gennaio 2018) richiama in primo luogo i lavori della VII Commissione della Camera dei deputati, che ha visto l'approvazione in data 21 ottobre 2014 di un documento, elaborato al termine di una indagine conoscitiva, molto larga e partecipata, sulle strategie per contrastare la dispersione scolastica.

L'insieme di esperienze, riflessioni e studi evidenzia in modo indiscutibile che per raggiungere obiettivi italiani ed europei in tema di inclusione sociale «contro il fallimento formativo occorrono azioni concertate con tutti gli attori educativi e sociali ed è indispensabile anche riavviare un dibattito onesto sui nodi concettuali, politici e operativi che attengono alla questione cruciale del perché tanto lavoro non ha prodotto un risultato da tutti auspicato» (p. 4). Il fallimento formativo in Italia rappresenta infatti sia un'emergenza nazionale che fenomeno multifattoriale. Il fallimento formativo è un fenomeno complesso, che unisce molti aspetti ed è strettamente collegato con ogni dimensione della crisi educativa presente in Italia; per essere contrastato richiede dunque «una strategia nazionale unitaria condivisa da tutti», 
che solleciti la centralità dei ragazzi, una inclusione precoce di bambini e ragazzi che sia educativa e sociale (pp. 5-6).

Vengono inoltre indicati come «saperi irrinunciabili» quelli che consentono di avere una base di conoscenze e competenze tale da potere esercitare la cittadinanza attiva e che permettono di riprendere gli studi nel corso della vita. In particolare, le competenze fondamentali in literacy e numeracy (leggere, scrivere e far di conto) vengono attestate in Italia dalle elaborazioni PISA (Programme for International Student Assessment) che, più o meno recenti, confermano una certa debolezza nelle "competenze irrinunciabili", in relazione al contesto familiare e territoriale, che risulta dunque dall'esclusione sociale e culturale. In particolare, «se si esaminano i ragazzi con i livelli più bassi di competenza nei saperi irrinunciabili della matematica di base e della lettura (low achievers), il 36\% dei quindicenni figli di poveri non raggiunge le competenze minime in matematica e il $29 \%$ in lettura e comprensione di semplici testi. E, ancora una volta, vi è un forte divario territoriale (PISA, 2015)» (MIUR, 2018, p. 10).

Nel Rapporto MIUR 2018 si sottolinea inoltre come la tenuta educativa e didattica sia migliore nella scuola primaria italiana, «che si mostra capace di assicurare risultati positivi in una competenza-chiave quale la lettura e comprensione dei testi ma che questi risultati non sono confermati dal percorso scolastico successivo, tra i 10 e i 15 anni» (p. 11).

Il Rapporto MIUR 2018 (p. 25), tra gli aspetti sui quali è necessario concentrare l'attenzione per contrastare il fallimento formativo, indica in particolare il «sostegno alla genitorialità e a ogni forma di integrazione multidimensionale delle famiglie e delle comunità di origine attraverso una regolare politica di ri-conoscimento e di perseveranza nel sostenere il binomio diritti-doveri»; «un'attenzione forte all'Italiano come primo veicolo di sapere». Viene inoltre riportata la definizione di "inclusione sociale" come «la misura in cui, con riguardo alle dimensioni principali del benessere, gli individui (e i gruppi) godono di standard minimi socialmente accettabili e presentano fra loro divari socialmente accettabili, avendo attenzione che il processo attraverso il quale questi risultati vengono ottenuti sia democratico ed equo» (Rapporto Barca, 2011, in MIUR, 2018, p. 27).

Denatalità e cambiamenti culturali e nel costume hanno indebolito il principio di autorità delle scuole e il senso del limite da parte delle famiglie e delle comunità anche a causa del ruolo fortemente diseducativo dei media: in questo nuovo scenario relazionale la scuola non può più contare su un retroterra di norme condivise e implicite per tutti, che evidenzia «una povertà dell'adulto, nelle sue funzioni educative, che probabilmente è anche dovuta 
a una vera crisi demografica». Da parte sua, la scuola è costretta quotidianamente a costruire, senza il sostegno sociale, una relazione educativa capace di intervenire sui codici di comportamento e sulla definizione delle regole sociali, fondando l'apprendimento «in modo attivo e vivo anziché su un sistema di sanzioni», cercando inoltre di «ricostruire esplicitamente e continuamente quel patto educativo tra adulti-genitori e adulti-docenti che una volta era implicito»: la scuola oggi è «il presidio educativo del Paese» (MIUR, 2018, pp. 32-33).

Quali raccomandazioni, dunque, per contrastare il fallimento formativo $\mathrm{o}$, meglio per assicurare il successo formativo? Prima di tutto la centralità dell'apprendimento, la coesione sociale e la via delle opportunità per tutti.

Tra le indicazioni del Consiglio dell'Unione Europea (28 giugno 2011, in MIUR, 2018, pp. 38-39): «misure di prevenzione», cioè affrontare i problemi strutturali; «misure di intervento», cioè migliorare la qualità dell'istruzione e della formazione; «misure di compensazione», attraverso nuove opportunità di ottenere una qualifica.

Tra le indicazioni dell'Assemblea Generale delle Nazioni Unite (Report 2014, in MIUR, 2018, pp. 39-40) tre gli obiettivi sostenibili: «tutti i minori hanno diritto ad apprendere, sperimentare, sviluppare capacità, talenti e aspirazioni; devono poter avere accesso all'offerta educativa di qualità; se poveri, devono poter essere destinatari di forti politiche pubbliche compensative e inclusive, a scuola e nella vita».

Tra le strategie di azione per combattere la dispersione scolastica indicate nel 2014 dalla VII Commissione della Camera erano stati individuati in particolare interventi in molteplici dimensioni nei confronti delle famiglie degli studenti a rischio, potenziandone i compiti e le capacità educative (MIUR, 2018, pp. 40-41).

A proposito di "buone pratiche", tra le cause del fallimento scolastico, viene evidenziato in Italia: un "sistema scolastico iper-standardizzato», a discapito delle pratiche di "scuola attiva", laboratoriale e partecipativa; la frammentazione delle discipline, una debole integrazione tra scuole, formazione professionale; occasioni formative e orientative di altri enti accreditati e debolezza dei percorsi scuola-lavoro; povere e poco diffuse procedure di orientamento e ri-orientamento nei territori (MIUR, 2018, pp. 41-42).

Per le attività di coordinamento tra diversi decisori, secondo il Rapporto MIUR 2018 (pp. 45-46, 50) infatti «il livello comunale appare di decisiva importanza, anche perché è, per il nostro ordinamento, il comune a essere responsabile dell'obbligo di istruzione». A livello di territorio occorre «una capacità di lettura che intercetti i casi singoli e sia in grado di recuperare storie e vicende», oltre a una crescita qualitativa della base informativa: la 
realizzazione dell'anagrafe nazionale degli studenti (ANS; Decreto Legislativo 15 aprile 2005, n. 76, art. 3) è infatti «uno strumento concepito non solo per fini statistici ma per l'intercettazione di studenti a rischio dispersione e per la costruzione di percorsi personalizzati», con educatori per il supporto educativo ad personam, secondo una logica win-win one to one.

Il Rapporto MIUR 2018 (pp. 47-54) si propone in particolare di sostenere la genitorialità; di estendere il tempo prolungato e pieno a partire dalle medesime aree evitando un ripetersi pomeridiano delle attività ordinarie integrandole, invece, sia con occasioni di apprendimento in ambito musicale, teatrale, dell'espressione grafico-pittorica, dello sport; di sostenere le esperienze di peer education; di promuovere forme di tutoring e mentoring; l'innovazione digitale e nuovi media di qualità; di investire sulla formazione in ingresso dei docenti; lo sviluppo delle competenze trasversali/life skills attraverso una didattica trasversale, laboratoriale e partecipativa; di garantire a ogni studente la certezza di seguire un percorso personalizzato per acquisire anche le competenze chiave di cittadinanza; di investire sull'orientamento informativo ed educativo come strumento, anche precoce, a supporto e accompagnamento delle scelte; di curare i luoghi educativi come luoghi comunitari; di prendere in considerazione una moratoria sulle bocciature e sperimentare, invece, un sistema rigoroso di crediti formativi.

\section{Riferimenti bibliografici}

(1948) Costituzione della Repubblica Italiana, testo disponibile al sito: http://www.quirinale.it/qrnw/costituzione/costituzione.html.

(1998-2017) Trattato sull'Unione europea (versione consolidata) e Trattato sul funzionamento dell'Unione europea (versione consolidata), Carta dei Carta dei Diritti Fondamentali dell'Unione Europea, Unione Europea.

Banfield E.C. (2010), Le basi morali di una società arretrata, ed. or. 1958, Il Mulino, Bologna.

Batini F. (2013), "Drop-out: storia di un rovesciamento", La ricerca, 13, testo disponibile al sito: http://www.laricerca.loescher.it/istruzione/593-drop-out-storia-di-un-rovesciamento.html.

Batini F. (2014), Drop-out, Fuorionda, Arezzo.

Batini F., Bartolucci M. e De Carlo M.E. (2017), "Fight Dispersion Through Education: The Results of the First Cycle of the NoOut Project", Mind, Brain and Education, XI, 4: 201212.

* Un ringraziamento particolare al Professor Sante Bucci, studioso e docente di pedagogia, il cui elevato profilo istituzionale e sociale ispira non soltanto questo lavoro ma anche la mia attività. 
Benvenuto G., a cura di (2011), La scuola diseguale. Dispersione ed equità nel sistema di istruzione e formazione, Anicia, Roma.

Bertonelli E. e Rodano G., a cura di (2003), Le riforme nella scuola italiana dal 1859 al 2003 - SCHEDE, con la consulenza di G. Chiosso e G. Tognon, testo disponibile al sito: http://www2.indire.it/materiali_dirigenti/1_bertonelli.pdf.

Bradley C.L. and Renzulli L.A. (2011), "The Complexity of Non-Completion: Being Pushed or Pulled to Drop Out of High School”, Social Forces, 90, 2: 521-545.

Bucci S., a cura di (2000), Professionalità e formazione universitaria degli insegnanti. Contributi per un'analisi comparativa, Armando, Roma.

Camera dei deputati - Commissione VII - Cultura, Scienza e Istruzione (2014), Resoconto stenografico Indagine conoscitiva sulle strategie per contrastare la dispersione scolastica, 21 ottobre 2014, testo disponibile al sito: http://documenti.camera.it/leg17/resoconti/commissioni/stenografici/pdf/07/indag/c07_dispersione/2014/10/21/leg.17.stencomm.data20141021.U1.com07.indag.c07_dispersione.0008.pdf.

Cantone R. e Caringella F. (2017), La corruzione spuzza. Tutti gli effetti sulla nostra vita quotidiana della malattia che rischia di uccidere l'Italia, Mondadori, Milano.

Catani M. (2018), "La nuova neuroanatomia funzionale delle connessioni cerebrali", Prolusione alla seduta inaugurale dell'anno accademico 2017-2018 dell'Accademia Anatomico-Chirurgica di Perugia, $216^{\circ}$ dalla sua fondazione, 15 febbraio 2018.

Farinelli G. (2017), La relazione educativa nella Costituzione Italiana, in Mollo G., a cura di, All'inizio è la relazione. Aspetti pedagogico-didattici, Aracne, Roma, pp. 113-122.

Fubini F. (2018), La maestra e la camorrista. Perché in Italia resti quello che nasci, Mondadori, Milano.

Galli della Loggia E. (2017), "L'abbandono della scuola", Corriere della Sera, 16 gennaio 2017.

Galli della Loggia E. (2010), L'identità italiana, ed. or. 1998, Il Mulino, Bologna.

Gratteri N. e Nicaso A. (2010), La malapianta. La mia lotta contro la 'ndrangheta, Mondadori, Milano.

Heckman J.J. (2013), Giving Kids a Fair Chance, Mit Press, Cambridge MA.

Levi Montalcini R. (1989), Elogio dell'imperfezione, ed. or. 1989, Garzanti, Milano.

Mastrocola P. (2011), Togliamo il disturbo. Saggio sulla libertà di non studiare, Guanda, Parma.

MIUR - Servizio statistico (2013), Focus "La dispersione scolastica", giugno 2013.

MIUR - Statistica e Studi (2017), Focus "La dispersione scolastica nell'a.s. 2015/2016 e nel passaggio all'a.s. 2016/2017”, novembre 2017, testo disponibile al sito: http://www.miur.gov.it/documents/20182/0/Focus+La+dispersione+scolastica/9bc1c11b-1c40-4029-87ba-40b8ba2bc2bf.

MIUR (2018), Una politica nazionale di contrasto del fallimento formativo e della povertà educativa. Cabina di regia per la lotta alla dispersione scolastica e alla povertà educativa, gennaio 2018, testo disponibile al sito: http://www.miur.gov.it/documents/20182/0/Rapporto+sul+contrasto+del+fallimento+formativo/7575f155-63f9479a-a77f-1 da743492e92?version=1.0.

Ong W. (1986), Oralità e scrittura. Le tecnologie della parola, ed. or. 1986, Il Mulino, Bologna.

ONU - Assemblea Generale delle Nazioni Unite (2014), Report of the Open Working Group of the General Assembly on Sustainable Development Goals, testo disponibile al sito: http://www.ohchr.org/EN/UDHR/Documents/UDHR_Translations/itn.pdf.

Patton G.C. and Viner R. (2007), "Pubertal transitions in health", The Lancet; 369: 1130-39.

Reboul O. (1977), Lo slogan, ed. or. 1975, Armando, Roma. 
Rumberger R.W. and Lamb S.P. (2003), “The early employment and further education experiences of high school dropouts: A comparative study of the United States and Australia", Economics of Education Review, 22, 4: 353-366.

Save the Children (2017), $8^{\circ}$ Atlante dell'infanzia a rischio. "Lettera alla scuola", Treccani, testo disponibile al sito: link https://www.savethechildren.it/atlante-dell-infanzia-rischio nov 2017.

UE, Commissione Barca/McCann (2011), Outcome indicators and targets -towards a new system of monitoring and evaluation in EU cohesion policy, giugno 2011.

Weil S. (1990), La prima radice. Preludio ad una dichiarazione dei doveri verso l'essere umano, trad. it., ed. or. 1949, SE, Milano. 


\section{Profili autori}

Laura Arcangeli, professore associato in Pedagogia e Didattica speciale presso l'Università degli Studi di Perugia. È delegata del Rettore per i servizi agli studenti con disabilità e/o con DSA dell'Università degli Studi di Perugia e Direttore del Corso di Specializzazione per le attività di sostegno nello stesso Ateneo. È autrice di diverse pubblicazioni sui temi dell'integrazione, inclusione, pedagogia e didattica speciale.

Roberta Balloni, ha conseguito la laurea in Lingue e letterature straniere presso l'Università degli Studi di Perugia. Ha avuto esperienze di insegnamento di lingue e culture straniere presso istituti di istruzione secondaria di secondo grado e scuole secondarie di primo grado. È attualmente iscritta al Corso di Laurea magistrale a ciclo unico in Scienze della Formazione Primaria presso l’Università degli Studi di Perugia.

Marco Bartolucci, post-doc researcher, Phd in neuroscienze. Tra gli ambiti di ricerca: prevenzione e contrasto della dispersione scolastica; empowerment cognitivo; processi di lettura e ascolto narrativo, relazione tra neuroscienze e apprendimento. Si interessa inoltre di software di riabilitazione cognitiva e strategie educative con devices di realtà aumentata. Tra le pubblicazioni: Dispersione scolastica. Ascoltare i protagonisti per comprenderla e prevenirla (a cura di, con F. Batini, FrancoAngeli, 2016).

Federico Batini, professore associato di Pedagogia Sperimentale presso il Dipartimento FISSUF dell'Università degli Studi di Perugia. Tra le ultime pubblicazioni in tema si segnalano i volumi: con S. Giusti (a cura di), Imparare dalla lettura (Loescher, 2013); con M. Bartolucci (a cura di), Dispersione scolastica. Ascoltare i protagonisti per comprenderla e prevenirla (FrancoAngeli, 2016); C'era una volta un pezzo di legno (FrancoAngeli, 2016); Dropout (Fuorionda, 2014 Premio Italiano di Pedagogia) e, tra gli 
altri, il contributo su Rivista (con M. Bartolucci e M. E. De Carlo): Fight Dispersion Through Education: The Results of the First Cycle of the NoOut Project, "Mind, Brain, and Education", (nov. 2017). Coordina il network internazionale di ricerca sulla dispersione IReNE. Dirige la Rivista Lifelong, Lifewide Learning. Ha fondato il movimento di volontari per la lettura LaAV. Ha ideato il metodo dell'orientamento narrativo.

Cecilia Bini, laureanda in Scienze dell'Educazione presso l'Università degli Studi di Perugia.

Cristiano Corsini, professore associato all'Università d'Annunzio di Chieti-Pescara, dove insegna Ricerca pedagogica e Progettazione educativa. Si occupa di formazione docenti, di ricerca valutativa e di indagini su efficacia ed equità in campo educativo. Ha pubblicato Differenze di genere nell'editoria scolastica (assieme a Irene D.M. Scierri, 2016), Valutare scuole e docenti, (2015, libro vincitore del Premio Nazionale di Pedagogia) e Il Valore Aggiunto in educazione. Un'indagine nella scuola primaria (2009).

Mina De Santis, professore associato di Didattica Generale presso l'Università degli Studi di Perugia. Nella stessa Università insegna anche Progettazione e organizzazione dei servizi per l'infanzia. Si occupa da anni di formazione degli insegnanti, di progetti di ricerca-formazione presso le scuole e i servizi socio-educativi e per la prima infanzia; fa parte del Centro di Documentazione, Aggiornamento e Sperimentazione sull'Infanzia della Regione Umbria. È componente delle società scientifiche SIPED, SIRD e SIREM.

Ylenia Di Vito, diplomata presso il Liceo delle Scienze Umane di Campobasso e laureanda in Scienze della Formazione Primaria presso l'Università degli Studi di Perugia.

Floriana Falcinelli, professore Ordinario di Didattica e tecnologie dell'Istruzione (SSD M_PED/03), Coordinatore del Corso di Laurea in Scienze della Formazione Primaria, Delegato del Rettore per l'e-learning, Responsabile Scientifico del Centro di Documentazione Infanzia della Regione Umbria.

Giovanna Farinelli, professore associato di Pedagogia generale e sociale presso il Dipartimento di Filosofia, Scienze Sociali, Umane e della Formazione dell'Università degli Studi di Perugia. I suoi interessi di ricerca riguar- 
dano la filosofia dell'educazione, l'etica e la pedagogia dello sport, la pedagogia sociale, l'educazione e la salute; ha partecipato a congressi nazionali e internazionali su temi relativi all'attività didattica e scientifica; tra le sue pubblicazioni: L'educazione come metafora della vita. Paul Ricoeur: la filosofia e l'educazione (ESI, Napoli, 2000); Pedagogia dello sport ed educazione della persona (Morlacchi, Perugia, 2005); Simone Weil e la paideia greca (Morlacchi, Perugia, 2008).

Leonardo Fasi, laureando in Scienze dell'Educazione presso l'Università degli Studi di Perugia.

Christian Christrup Kjeldsen, professore associato e direttore del dipartimento di Scienze dell'Educazione, Università di Aarhus, Danimarca. Autore di numerose pubblicazioni scientifiche, si occupa principalmente di contenuto dei programmi di studio e formazione, sviluppo delle competenze chiave; gruppi vulnerabili nell'istruzione e nella formazione; disabilità/bisogni speciali, educazione inclusiva; istruzione e migrazione; abbandono scolastico; monitoraggio dell'equità nell'istruzione e nella formazione; istruzione e formazione della forza lavoro professionale.

Laura Lana, studentessa attualmente iscritta al corso di laurea in Scienze della Formazione Primaria presso l'Università degli Studi di Perugia. Precedentemente ha conseguito una laurea magistrale in Lettere Moderne presso l'Ateneo perugino. Ha collaborato con quotidiani e riviste in qualità di giornalista, dal 2011 è regolarmente iscritta all'Ordine dei pubblicisti dell'Umbria.

Annalisa Morganti, professore associato di Didattica e Pedagogia Speciale presso l'Università di Perugia. Lavora da anni in ambito nazionale ed europeo a ricerche sui temi dell'educazione socio-emotiva e dell'evidence-based education applicata all'inclusione scolastica. È Chair dello Europen Network for Social and Emotional Learning, fondatrice e coordinatrice del gruppo internazionale di ricerca "Special Interest Group on Assessment in Social and Emotional Learning”.

Elisa Morini, laureanda in Scienze dell'Educazione presso l'Università degli Studi di Perugia.

Mariangela Nitti, dottore di ricerca in Scienze della Mente e delle Relazioni Umane. Dal 2009 si occupa di metodologie statistiche applicate alla ricerca 
sociale ed educativa e alla psicometria. Ha ricoperto incarichi di docenza presso i corsi di laurea di Scienze e Tecniche Psicologiche, Scienze Politiche e delle Relazioni Internazionali, Sociologia e Ricerca Sociale dell'Università di Salento e presso enti di formazione privati.

Tra i temi di ricerca, i modelli di analisi statistica multivariata per la misurazione della soddisfazione e la valutazione dell'impatto di politiche ed interventi in ambito sociale.

Agnese Rosati, afferente al Dipartimento di Filosofia, Scienze Sociali, Umane e della Formazione, insegna Pedagogia generale nei CdS dell'Università degli Studi di Perugia. Responsabile della Qualità per il Corso in Scienze dell'educazione, è parte di équipe internazionali di ricerca educativa ed è membro di comitati scientifici per collane editoriali e riviste. Ha pubblicato monografie e scritti collettanei per gli editori Anicia e Aracne (Roma), Pensa (Lecce) e Liguori (Napoli). È Visiting Research presso l'Università di Salamanca e Visiting Professor and Research per la Escola Superior de Educação of Politécnico de Lisboa.

Rosario Salvato, professore associato presso il Dipartimento FISSUF dell'Università degli Studi di Perugia. Insegna Progettazione e organizzazione dei servizi socio-educativi e Modelli didattici per l'educazione permanente. Ha fatto parte del Comitato scientifico per la elaborazione del Secondo Piano sociale 2010/12 della Regione Umbria, il più importante strumento di programmazione regionale in materia di politiche socio-educative.

Moira Sannipoli, ricercatrice in Pedagogia e Didattica Speciale presso l'Università degli Studi di Perugia. È responsabile del Centro di Documentazione, Aggiornamento e Sperimentazione sull'Infanzia della Regione Umbria. È autrice di diverse pubblicazioni sui temi dell'infanzia, inclusione, diversità, differenze ed ha condotto numerose ricerche ed esperienze formative su queste tematiche. Ha ricevuto il premio Siped 2017 per il volume Diversità e differenze nella prospettiva coevolutiva (FrancoAngeli, Milano, 2015).

Irene Dora Maria Scierri, laureata in Scienze Pedagogiche e Progettazione Educativa all'Università degli Studi di Catania, dove ha collaborato con la cattedra di Pedagogia Sperimentale. Nel 2017 è assegnista di ricerca presso l'Università degli Studi di Perugia, dove attualmente continua a svolgere attività di ricerca all'interno del Dipartimento di Filosofia, Scienze Sociali, Umane e della Formazione. Si occupa di formazione docenti sulla didattica 
e la valutazione per competenze. Tra le pubblicazioni: Differenze di genere nell'editoria scolastica (con C. Corsini; Nuova Cultura, Roma, 2016).

Alessandra Sisto, laureata in Lingue e Letterature Straniere Moderne presso l'Università La Sapienza di Roma, laureanda in Scienze dell'Educazione presso l'Università degli Studi di Perugia e insegnante di yoga.

Adriana Timpone, laureata magistrale in Scienze dell'Educazione e Formazione. Ha collaborato con la cattedra di pedagogia sperimentale dell'Università degli Studi di Perugia, con il CdS in Scienze dell'Educazione e della Formazione di Sapienza, Università di Roma ed altri enti di ricerca. In tali contesti ha svolto attività di ricerca sull'alternanza scuola-lavoro, la dispersione scolastica, l'orientamento e la didattica per competenze. Attualmente è collaboratore tecnico presso INVALSI, area rilevazioni nazionali. 


\section{QUESTO LIBRO TI È PIACIUTO?}

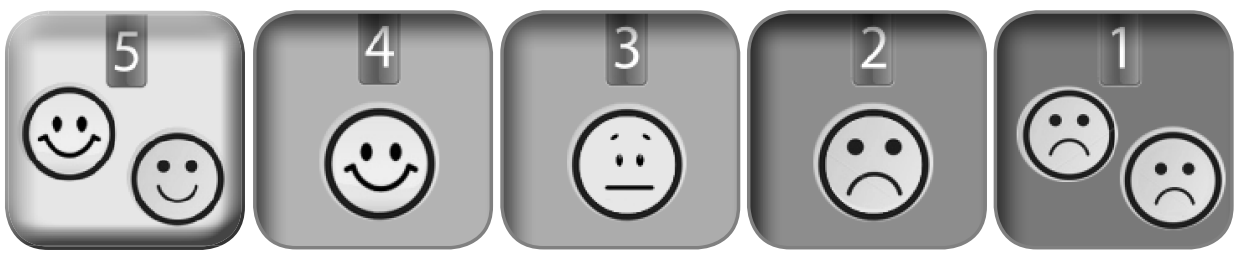

\section{Comunicaci il tuo giudizio su:}

www.francoangeli.it/latuaopinione.asp

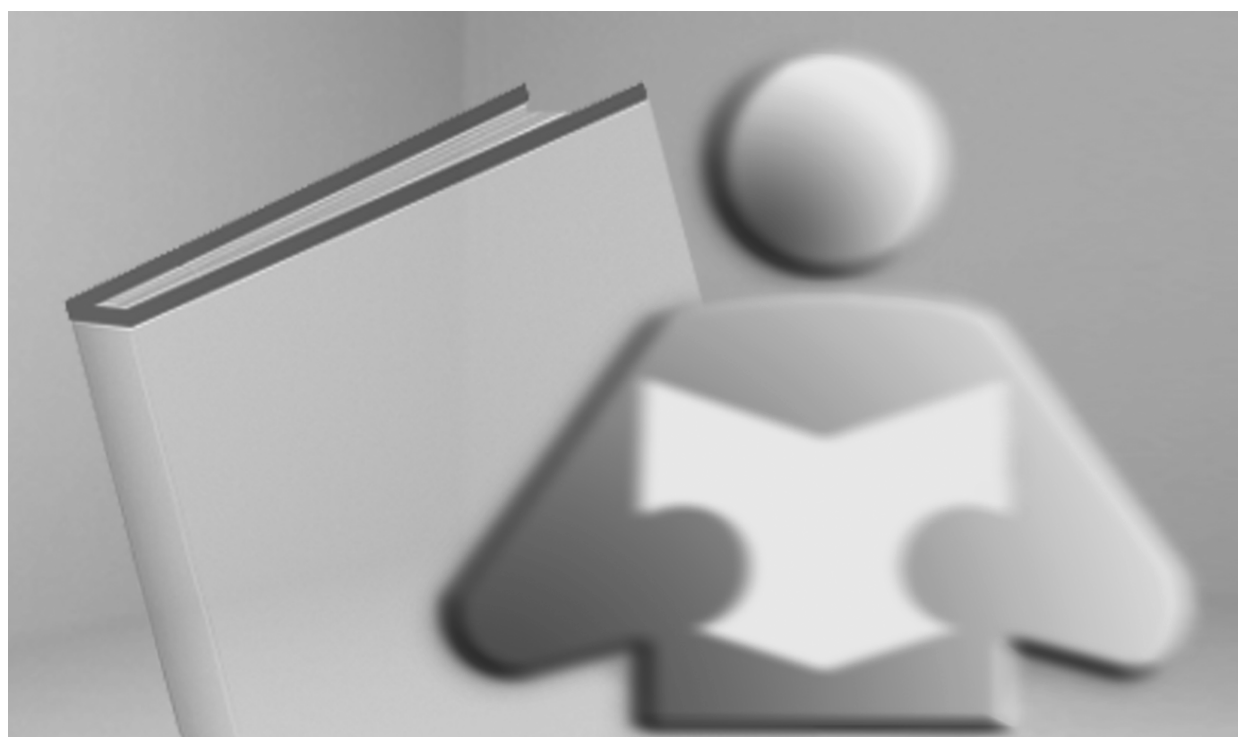

\section{VUOI RICEVERE GLI AGGIORNAMENTI SULLE NOSTRE NOVITÀ NELLE AREE CHE TI INTERESSANO?}

3 Seguici in rete $20 \begin{aligned} & \text { Sottoscrivi } \\ & \text { i nostri feed RSS }\end{aligned}$

\section{FrancoAngeli}


Lettura e dispersione costituiscono, in modi diversi, due punti cruciali della realtà scolastica e della ricerca educativa.

La dispersione scolastica è un'emergenza sociale, il cui contrasto è reso più complesso anche dalle differenti modalità di contabilizzazione e dai differenti target presi in considerazione. Per comprendere a fondo la dispersione occorre ascoltare il punto di vista di tutti gli attori coinvolti; per affrontarla con approccio preventivo occorre agire in ottica sistemica, attribuendo un ruolo centrale all'esperienza scolastica e al ruolo fondamentale che i docenti ricoprono nell'attività di prevenzione della dispersione, attraverso le loro pratiche didattiche, valutative e relazionali. La lettura si configura, in questo senso, come una pratica capace di esercitare una funzione rilevante di supporto al successo formativo e all'empowerment dei soggetti.

Nel libro vengono evidenziati i molteplici effetti cognitivi ed emotivi della lettura e, più in generale, le diverse modalità in grado di prevenire e contrastare la dispersione scolastica. Il volume riveste dunque un doppio interesse: da un lato, aiutare la comprensione della dispersione scolastica e la percezione che se ne ha sul campo; dall'altro, individuare interventi di prevenzione e recupero efficaci, con particolare attenzione alla lettura ad alta voce e all'orientamento narrativo.

Contributi di: Laura Arcangeli, Roberta Balloni, Marco Bartolucci, Federico Batini, Cecilia Bini, Cristiano Corsini, Mina De Santis, Ylenia Di Vito, Floriana Falcinelli, Giovanna Farinelli, Leonardo Fasi, Christian C. Kjeldsen, Laura Lana, Annalisa Morganti, Elisa Morini, Mariangela Nitti, Agnese Rosati, Rosario Salvato, Moira Sannipoli, Irene D.M. Scierri, Alessandra Sisto, Adriana Timpone.

Irene Dora Maria Scierri, laureata in Scienze pedagogiche e Progettazione educativa all'Università degli Studi di Catania, ha collaborato con la cattedra di Pedagogia sperimentale. Nel 2017 è assegnista di ricerca presso I'Università degli Studi di Perugia, dove attualmente continua a svolgere attività di ricerca. Si occupa di formazione docenti sulla didattica e la valutazione per competenze. Tra le pubblicazioni: Differenze di genere nell'editoria scolastica (con C. Corsini; Nuova Cultura, Roma, 2016).

Marco Bartolucci, post-doc researcher, Phd in neuroscienze. Tra gli ambiti di ricerca: prevenzione e contrasto della dispersione scolastica; empowerment cognitivo; processi di lettura e ascolto narrativo, relazione tra neuroscienze e apprendimento. Si interessa inoltre di software di riabilitazione cognitiva e strategie educative con devices di realtà aumentata. Tra le pubblicazioni: Dispersione scolastica. Ascoltare i protagonisti per comprenderla e prevenirla (a cura di, con F. Batini, FrancoAngeli, Milano 2016).

Rosario Salvato, professore associato presso il Dipartimento FISSUF dell'Università degli Studi di Perugia, insegna Progettazione e organizzazione dei servizi socio-educativi e Modelli didattici per l'educazione permanente. Ha fatto parte del Comitato scientifico per la elaborazione del Secondo Piano sociale 2010/12 della Regione Umbria, il più importante strumento di programmazione regionale in materia di politiche socio-educative.

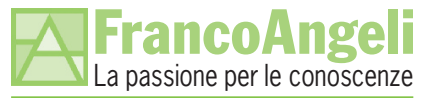

